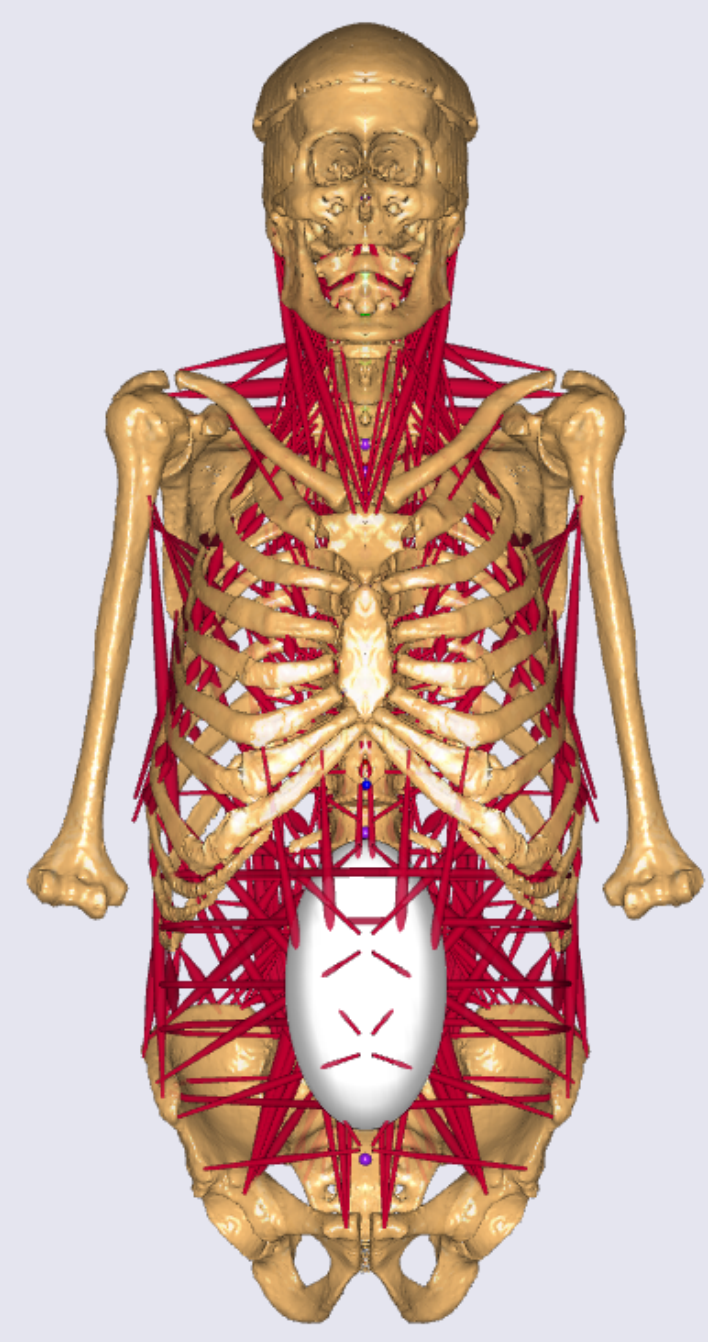

\title{
Twente Spine Model
}

Development, validation, and application of a complete and coherent musculoskeletal model of the human spine

Riza Bayoglu 


\section{TWENTE SPINE MODEL}

DEVELOPMENT, VALIDATION, AND APPLICATION OF A COMPLETE AND COHERENT MUSCULOSKELETAL

MODEL OF THE HUMAN SPINE

Riza Bayoglu 



\title{
TWENTE SPINE MODEL
}

\section{DEVELOPMENT, VALIDATION, AND APPLICATION OF A COMPLETE AND COHERENT MUSCULOSKELETAL MODEL OF THE HUMAN SPINE}

\author{
DISSERTATION
}

to obtain

the degree of doctor at the University of Twente, on the authority of the rector magnificus, prof.dr. T.T.M. Palstra, on account of the decision of the Doctorate Board, to be publicly defended on Thursday the $23^{\text {th }}$ of May 2019 at 16.45 p.m.

by

Riza Bayoglu

born on the $9^{\text {th }}$ of November 1986

in Istanbul, Turkey 
This dissertation has been approved by:

Supervisors:

prof.dr.ir. H.F.J.M. Koopman

prof.dr.ir. N.J.J. Verdonschot

Co-supervisor:

dr.ir. J.J. Homminga

Cover design: Riza Bayoglu

Lay-out: Riza Bayoglu

Printed by: Ipskamp printing

ISBN: 978-90-365-4771-0

DOI: $10.3990 / 1.9789036547710$

(C) 2019 by Riza Bayoglu, Enschede, The Netherlands.

All rights reserved. No parts of this thesis may be reproduced, stored in a retrieval system or transmitted in any form or by any means without permission of the author.

Alle rechten voorbehouden. Niets uit deze uitgave mag worden vermenigvuldigd, in enige vorm of op enige wijze, zonder voorafgaande schriftelijke toestemming van de auteur. 


\section{GRADUATION COMMITTEE:}

Chairman/secretary:

prof.dr.ir. G.P.M.R. Dewulf

University of Twente

Supervisors:

prof.dr.ir. H.F.J.M. Koopman

University of Twente

prof.dr.ir. N.J.J. Verdonschot

University of Twente

Co-supervisor:

dr.ir. J.J. Homminga

University of Twente

Members:

prof.dr.ir. G.J. Verkerke

University of Twente

prof.dr.ir. C.H. Slump

University of Twente

prof.dr.ir. T.M.T. Villa

Politecnico di Milano

prof.dr.ir. M.De Kleuver

Radboud University

dr.ir. B. van Rietbergen

Eindhoven University of Technology 
The work presented in this dissertation was conducted at the Department of Biomechanical Engineering at the University of Twente and was supported by a grant from fonds NutsOhra (1302-032).

The printing of this dissertation was financially supported by the Department of Biomechanical Engineering at the University of Twente. 
Summary $\quad$ ix

Samenvatting $\quad$ xiii

1 General introduction 1

2 Twente Spine Model: A complete and coherent dataset for musculoskeletal modeling of the lumbar region of the human spine $\quad 7$

3 Twente spine model: A complete and coherent dataset for musculoskeletal modeling of the thoracic and cervical regions of the human spine $\quad 23$

4 Twente Spine Model: A thorough investigation of the spinal loads in a complete and coherent musculoskeletal model of the human spine

5 Sensitivity of muscle and intervertebral disc force computations to variations in muscle attachment sites

6 A coupled set of kinematic and electromyographic data for validation of spinal musculoskeletal models

7 Combining musculoskeletal and finite element modeling to predict spinal

failure under physiological load cases.

An exploratory study

8 General discussion $\quad 107$

\begin{tabular}{ll}
\hline A Appendix A & 119
\end{tabular}

\begin{tabular}{ll}
\hline B Appendix B & 127
\end{tabular}

$\begin{array}{ll}\text { Bibliography } & 133\end{array}$

\begin{tabular}{ll}
\hline Acknowledgments & 151
\end{tabular}

$\begin{array}{ll}\text { Biography } & 153\end{array}$

$\begin{array}{ll}\text { Publications } & 155\end{array}$ 

$\mathrm{T}$ he human spine is a complex biologic structure composed of several articulating bones, intervertebral discs, and ligaments. The spine interacts with the shoulderarm complex and lower extremity and carries important functions such as protecting the spinal cord, supporting the trunk, and providing motion. While performing these functions, the spine is exposed to considerable loads which might affect its performance and lead to musculoskeletal and neuromuscular complications. Understanding the nature of the loads acting on the spinal tissues can assist with diagnosing and treating spinal pathologies, thus assessment of the spinal loads has been the focus of much research. In the past, several studies measured in vivo spinal loads for most activities of daily life through intradiscal pressures or telemetrized implants. Unfortunately, these measurements were limited to some thoracic and lumbar levels due to difficulties primarily associated with ethical considerations. A comprehensive assessment of the spinal loads throughout the spine can advance our understanding of its functioning but is largely unavailable.

Musculoskeletal modeling offers a non-invasive means to estimate in vivo spinal loads and can thus provide clinical insights into the spine's functioning. The primary objective of this thesis was to develop a validated, complete and coherent musculoskeletal model of the entire human spine for investigating the spinal loads. To achieve this goal, in Chapters 2 \& 3 an anatomical dataset (the Twente Spine Dataset) including necessary musculoskeletal parameters for creating this model was measured. This dataset was obtained by medical imaging and detailed dissection of a male cadaver. Spinal and abdominal muscles located in the ribcage and cervical, thoracic, and lumbar regions were identified and divided into several muscle-tendon elements. For each element, locations of the attachment sites at the origin, insertion, and via points were digitized, and its morphology was measured. The morphological parameters consisted of the fiber length, tendon length, sarcomere length, optimal fiber length, pennation angle, mass, and physiological cross-sectional area. New quantitative data were reported for several muscles such as rotatores, multifidus, serratus posterior inferior, levatores costarum, spinalis, semispinalis, subcostales, transversus thoracis, and intercostales. Muscle parameters reported in this dataset were within the range of data found in earlier studies. This dataset facilitates the development of a coherent musculoskeletal model for the entire human spine and prevents uncertainties intrinsic in combining musculoskeletal data from different studies. In essence, this dataset makes up the first complete and consistent atlas for modeling the human spine.

In Chapter 4 a complete and coherent musculoskeletal model of the entire human spine (the Twente Spine Model) was developed based on the previously acquired anatomical 
dataset. In this model, cervical, thoracic, and lumbar vertebrae, a flexible ribcage, and comprehensive muscular anatomy were incorporated. This spine model in principle represents a subject-specific musculoskeletal system and, unlike generic models, does not require combining anatomical data from several studies. An inverse dynamics based static optimization routine minimizing muscle fatigue was used for calculating muscle and joint forces during basic neck and trunk movements. For validation of the predicted internal loads, quasi-static trunk tasks as measured in previous in vivo studies were simulated, and calculated intradiscal pressures at thoracic and lumbar discs and normalized resultant loads were compared. In addition, disc compression forces were calculated during upright standing, flexion, extension, lateral bending, axial rotation and were reported as percentages of the total body weight. Disc compression forces, in general, increased caudally and with increasing trunk motion, yet decreased at the lumbar and lower thoracic levels in extension. The cervical compression forces were lower than those of the thoracolumbar discs during neck tasks. Although there were some discrepancies, the results, in general, agreed well with previously measured in vivo spinal loads. This indicated the first validation of the Twente Spine Model in predicting the internal loads at the intervertebral discs during basic activities of daily life.

In Chapter 5, the sensitivity of muscle and intervertebral disc force computations against potential errors in modeling muscle attachment sites (muscle origin, insertion, and via points) were investigated. For this, every muscle attachment location was perturbed in the Twente Spine Model during upright standing, flexion, lateral bending, and axial rotation of the trunk. The changes in the T6/T7, T12/L1, and L4/L5 disc forces were analyzed, and an overall sensitivity index value was calculated for every perturbed muscle. Most of the muscles that were studied resulted in minor changes (less than 5\%) in disc forces. However, certain muscle groups had a large influence. For example, fluctuations in the quadratus lumborum caused changes in the shear forces as high as $353 \%$ and changes in the compressive forces as high as $17 \%$. Musculoskeletal simulations identified certain muscles in the ribcage and lumbar spine as being more sensitive for calculating muscle and disc forces. The findings suggest that modeling muscle attachment sites simply based on anatomical illustrations might lead to an erroneous evaluation of the internal forces.

In Chapter 6, electromyographic activities and trunk movements during isometric and dynamic trunk activities were simultaneously measured. Although differences were observed between women and men and between body heights, consistent muscle activation trends were found in most static and dynamic exertions. Measured trunk tasks could be isometrically or dynamically simulated in inverse dynamics based spinal musculoskeletal models, and predicted and measured muscle activations could then be compared to verify musculoskeletal predictions.

Finally, in Chapter 7, musculoskeletal and patient-specific finite element models were used 
in combination to investigate if modeling more physiological load regimes (compared to simpler loading regimes) can significantly affect the vertebral fracture risk prediction. The Twente Spine Model was scaled to the body height and weight of a patient who had spinal metastasis and was used to extract the loading and boundary conditions at the L2/L3 joint during physiological trunk movements. These boundary conditions were implemented in a finite element model of the L3-L5 spinal unit for studying its mechanical behavior. Results indicated that only trunk flexion caused plasticity at the L4 vertebra and that the disc force during $45^{\circ}$ flexed position was significantly lower than the axial force which resulted in the same plasticity level. This implied that the metastasized vertebra was likely to fail under flexion loads than under only a compression load. 



\section{SAMENVATTING}

$\mathrm{D}$ e menselijke wervelkolom is een complexe biologische structuur, die is samengesteld uit verschillende articulerende botten, tussenwervelschijven, en ligamenten. De wervelkolom heeft interactie met het schouder-arm complex en met de onderste extremiteiten en heeft daarnaast belangrijke rollen in de bescherming van het ruggenmerg, de ondersteuning van de romp, en het mogelijk maken van beweging. Tijdens het uitvoeren van deze functies ondergaat de wervelkolom aanzienlijke belastingen die kunnen leiden tot musculoskeletale aandoeningen en neuromusculaire complicaties. Een juist en gedetailleerd beeld van de aard en werking van de belastingen op de wervelkolom zou helpen bij het diagnosticeren en behandelen van spinale aandoeningen. Een degelijke beoordeling van de belasting van de wervelkolom is derhalve de focus geweest van veel onderzoek. Verschillende studies hebben, middels intradiscale druk of telemetrisch implantaten, in vivo wervelkolom belastingen voor dagelijkse activiteiten gemeten. Uit, met name, ethische overwegingen zijn deze metingen tot op heden beperkt gebleven tot enkele thoracale en lumbale niveaus. Een uitgebreide beoordeling van de spinale belastingen doorheen de gehele wervelkolom, die het begrip van het functioneren zou bevorderen, is in wezen niet beschikbaar.

Musculoskeletale modellering biedt een niet-invasieve manier omin vivo wervelkolom belastingen te bepalen en kan zo klinische inzicht bieden in het functioneren van de wervelkolom. Het primaire doel van dit promotiewerk was de ontwikkeling van een compleet, coherent, en gevalideerd musculoskeletaal model van de gehele menselijke wervelkolom, bruikbaar voor onderzoek naar de spinale belasting. In Hoofdstuk 2 \& 3 , werd een anatomisch dataset (de Twente Spinal Dataset), met alle voor het model noodzakelijke musculoskeletale parameters, opgebouwd. Die benodigde data is verkregen middels medische beeldvorming en gedetailleerde dissectie van een mannelijk kadaver. De spieren van de rug en buik, gelegen in de ribbenkast en cervicale, thoracale, en lumbale regio's werden geïdentificeerd en verdeeld in verschillende spier-peeselementen. Voor elk element werden de locaties en van origo, insertio, en via-punten gedigitaliseerd en de morfologie vastgesteld. De morfologische spierparameters bestonden uit de vezellengte, peeslengte, sarcomeer lengte, optimale vezellengte, pennatiehoek, massa, en fysiologische dwarsdoorsnede oppervlak. Niet eerder gemeten kwantitatieve gegevens werden gerapporteerd voor verschillende spieren zoals rotatores, multifidus, serratus posterior inferior, levatores costarum, spinalis, semispinalis, subcostales, transversus thoracis, en intercostales. De spierparameters in deze nieuwe dataset waren vergelijkbaar met die uit eerdere studies. Deze dataset maakt het mogelijk een coherent musculoskeletaal model voor de gehele menselijke romp te ontwikkelen, zonder de onzekerheden die resulteren uit het combineren van musculoskeletale gegevens van verschillende wervelkolommen. Deze dataset representeert de eerste compleet 
en consistente atlas voor het modelleren van de menselijke wervelkolom.

In Hoofdstuk 4, werd een compleet en coherent musculoskeletaal model van de gehele menselijke wervelkolom (het Twente Spine Model) ontwikkeld op basis van de eerder verworven anatomische dataset. Het model bevat cervicale, thoracale, en lumbale wervels, een flexibele ribbenkast, en een uitgebreide spier anatomie. Dit model representeert in de eerste plaats een persoons specifiek musculoskeletaal systeem en bevat, in tegenstelling tot generieke modellen, geen combinatie van anatomische gegevens van verschillende wervelkolommen. Een op inverse dynamica gebaseerde statische optimalisatieroutine, die spiervermoeidheid minimaliseert, werd gebruikt voor het berekenen van spier- en gewrichtskrachten tijdens basale nek en romp bewegingen. Ter validatie van de voorspelde interne belastingen, werden quasi-statische taken, zoals bemeten in eerdere in vivo studies, gesimuleerd en de aldus berekende intradiscale drukken vergeleken met de in vivo gemeten waarden. Daarnaast werden de disc-compressie krachten tijdens rechtop staan, flexie, extensie, laterale buiging, en axiale rotatie van de wervelkolom berekend en gerapporteerd als percentages van het totale lichaamsgewicht. In het algemeen, namen disc-compressie krachten toe richting caudaal. In het algemeen, namen disc-compressie krachten eveneens toe en met de amplitude van de rompbeweging, behalve in extensie waar ze, op de lumbale en lagere thoracale niveaus, afnamen. De cervicale compressiekrachten waren lager dan die in de thoracale tijdens nek taken. Ondanks enkele afwijkingen, kwamen de wervelkolom belastingen goed overeen met de eerder gemeten in vivo spinale belastingen. Dit representeert een eerste validatie van Twente Spine Model voor het voorspellen van de belastingen op de tussenwervelschijven tijdens de basisactiviteiten van het dagelijks leven.

In Hoofdstuk 5, werd de gevoeligheid van de berekende belastingen, in spieren en tussenwervelschijven, voor potentiele fouten in de locaties van spieraanhechting (origo, insertio, en via-punten) onderzocht. Hiervoor werd de locatie van elke spieraanhechting in het Twente Spine Model gevarieerd tijdens rechtop staan, flexie, laterale buiging, en axiale rotatie. De resulterende fluctuaties in de belasting van de tussenwervelschijven (T6/T7, T12/L1, en L4/L5) werden geanalyseerd en weergegeven in een gevoeligheidsindex per gevarieerde spier. Hoewel de meerderheid van de bestudeerde spierfluctuaties slechts kleine veranderingen $(<5 \%)$ in de berekende tussenwervel krachten lieten zien, lieten bepaalde spiergroepen grote veranderingen in de berekende tussenwervel krachten zien. Variaties in de quadratus lumborum, bijvoorbeeld, resulteerden in veranderingen van de schuifkracht tot $353 \%$ en veranderingen van de compressie krachten van $17 \%$. De musculoskeletale simulaties lieten zien dat bepaalde spieren in de ribbenkast en lumbale wervelkolom beduidend meer invloed hadden op de berekende spier en disc krachten dan andere spieren. De bevindingen suggereren dat het modelleren van de locatie van spieraanhechting simpelweg op basis van anatomische illustraties kan leiden tot onjuiste interne krachten. 
In Hoofdstuk 6, werden, tijdens isometrisch en dynamisch romp activiteiten, simultaan, de spieractiviteit van de belangrijkste rompspieren en de bewegingen gemeten. Hoewel er verschillen in activeringspatronen tussen vrouwen en mannen en tussen verschillende lichaamslengtes waren, werden, bij de meeste statische en dynamische inspanningen, consistente trends in spieractivatie gevonden. De gemeten romptaken konden isometrisch of dynamisch worden gesimuleerd op basis van musculoskeletale modellen van de romp, en de voorspelde en gemeten spieractivaties konden worden vergeleken om musculoskeletale voorspellingen te verifiëren.

Ten slotte, in Hoofdstuk 7, werden musculoskeletale en patiënt-specifieke eindige elementenmodellen gebruikt om te onderzoeken of het gebruiken van meer fysiologische belasting de voorspellingen van de wervelfractuurrisico's significant kan beïnvloeden. Met behulp van het Twente Spine Model, geschaald naar lichaamslengte en gewicht van een patiënt met spinale metastase, werden de belastingen van het L2/L3 gewricht tijdens fysiologische rompbewegingen berekend. Deze belasting werden vervolgens geïmplementeerd in een eindig elementenmodel van het L3-L5 segment voor het bestuderen van het mechanische gedrag. De resultaten lieten zien aan dat alleen rompflexie falen veroorzaakte bij de L4 wervel en dat de discus krachten tijdens $45^{\circ}$ flexie significant lager waren dan de axiale kracht, die resulteerde in hetzelfde falingsniveau. Dit impliceerde dat de gemetastaseerde wervel waarschijnlijk faalt vanwege de flexiebelastingen en niet vanwege de compressiebelasting. 



\section{GENERAL INTRODUCTION}

This chapter provides a brief introduction to the topic and describes the motivations for this thesis work. Furthermore, an outline of the thesis is presented. 


\subsection{Spine biomechanics}

The spine is a complex unit of the human skeleton system consisting of several articulating bones and intervertebral discs. Morphologically, it acts as a bridge between the upper and lower extremities and maintains essential functions such as supporting the body weight, protecting the spinal cord and aiding in locomotion. Mechanically speaking, a good spinal flexibility to rigidity ratio is required for performing these duties effectively. These competing requirements render the spine vulnerable to a broad range of diseases and complications, such as back pain and vertebral fractures, that decrease the quality of life. As a result, the human spine has been the focus of many clinicians and biomechanical researchers.

The investigation of internal loads offers means to enhance our understanding of the spine's functioning and hence can improve clinical decision making. For example, muscle and joint forces measured during trunk exertions can be used to diagnose and treat neuromuscular disorders [1, 2]. Previously, internal loads in the spine have been measured only in a few groups of studies which reported intervertebral disc pressures or the forces and moments acting on instrumented-vertebral implants [3-[11]. These measurements were available for a wide variety of daily activities but were limited to the thoracic and lumbar regions. Disc compressive forces can be estimated from measured pressures [12]. However, shear forces at the intervertebral discs or muscle forces can not be extracted from these data. Furthermore, electrical activities of major spine muscles have been previously quantified to study muscle coordination strategies employed in healthy and pathological spines [13, 14]. It was previously discussed that non-invasive surface electromyography (sEMG) may not be very reliable for measuring deeper muscles and is bound to technical difficulties such as crosstalk [15].

Despite previous efforts in spinal research, our understanding of the in vivo mechanical function remains largely limited. The lack of sufficient knowledge is in part due to the spine's complex architecture and ethical barriers associated with invasive measurements on living subjects. A detailed assessment of the internal loads including all spinal intervertebral disc levels thorough in silico modeling would benefit our health care, for example with an improvement of the diagnostic methods, injury prevention [16], optimization of surgical interventions [17], and implant design [18] but is lacking. Computational models of the musculoskeletal system may provide clinically useful insights into its in vivo function since they facilitate to calculate the internal loads in the tissues during locomotion. Intricate structures such as the spine which is difficult or impossible to study in vivo using experimental techniques may be studied in detail [19]. For example, the consequences of removing paraspinal muscles as practiced during posterior spinal interventions may be evaluated before the actual surgery to plan an efficient approach [17]. 


\subsection{Computational modeling}

Early studies modeled passive structures of the spine by a combination of rigid bodies, springs, and dashpots with some degrees-of-freedom ( $\mathrm{DoF}$ ) and solved the governing equations of motion for prescribed geometrical configurations [19-23]. They discussed that the deformations of the ligaments and intervertebral discs can be calculated for physiological motions by simulating experimentally measured ranges of motion of the vertebral column. These early works are useful to understand the basics of the kinematics and kinetics of the spine, however, they are not suitable to study the relationship between muscle function and internal loading. Since active structures such as muscles were not represented, their clinical application is limited.

Various musculoskeletal representations of the spine have been presented in the literature. These active models utilized forward or inverse dynamics schemes for calculating the movement patterns or internal loads, respectively [24]. The applicability of the forward dynamics method is restrained since it requires muscle excitations or joint torques as inputs but these are not readily available. On the contrary, the inverse dynamics method uses measured joint kinematics to compute muscle and joint forces. Because spinal kinematics can be non-invasively measured, this method has been preferred in a greater number of the models aimed to answer basic or applied research questions. In active musculoskeletal models, force production mechanisms in the skeletal muscles have been represented with varying complexity from sarcomere to fiber levels. Because the number of muscles in our body is larger than the number of equilibrium equations of motion, such models use optimization routines for computing muscle activations. These routines aim to mimic the working principles of the central nervous system. In this scheme, muscle recruitments during trunk exertions are predicted by usually minimizing metabolic energy consumption or muscle fatigue with quadratic or cubic polynomial cost functions [25].

Several authors have investigated the spinal loads in their computational models employing inverse dynamics based optimization. These models represented the lumbar [26-32], thoracolumbar [33, 34], cervical [35, 36], or cervico-thoraco-lumbar [37] regions of the human spine and have been advanced in complexity for representing generic or subject-specific systems. There are, however, several problems one encounters in the construction of spinal musculoskeletal models, and the currently available models have been no exception to these. The majority of the existing generic models pieced musculoskeletal data from a group of earlier dissection studies and made assumptions on missing data when these were unavailable. Subsequently, generic models will necessitate complex scaling between the muscle and skeletal anatomy of the cadavers studied, nevertheless a physiologically sound-scaling protocol has never been applied to or even developed for these data. As such, generic models are likely to fail to embody morphologically realistic spines although the intention is to rep- 
resent average populations. In comparison to generic models, subject-specific models enclose more realistic representations of the muscle and skeletal anatomy [38, 39]. Furthermore, the majority of these models focused on a single region of the spine and idealized the thorax or neck as lumped rigid bodies. Although incorporating the thorax as a single rigid body when calculating the lumbar loads was previously studied for flexion [40], it is yet unclear how muscle and joint predictions would be altered during other trunk movements.

A large number of the previous models used earlier anatomical illustrations to construct muscle paths and did not incorporate deeper muscles. These illustrations were obtained by visual inspection of the muscle attachments from several cadavers and prepared to indicate average likely locations. To implement subject-specific muscle architecture, recent studies developed novel methods to adjust muscle paths and cross-sectional areas of major trunk muscles through medical images [31, 33, 41]. Accurate measurement of muscle attachment sites from medial images is still challenging, especially for smaller and deeper muscles [42]. Finally, muscle parameters-such as physiological cross-sectional area and optimal fiber length-which are influential in model predictions [43] can not be reliably measured from two-dimensional medical images [42, 44]. Despite these previous efforts, there is a need for a musculoskeletal model of the entire human spine based on a complete and coherent anatomical dataset to further promote our understanding of the spine's in vivo functioning.

\subsection{Thesis outline}

The main goal of this dissertation is to develop a validated, complete, and coherent musculoskeletal model of the human spine for studying internal spinal loads during physiological movements. The research output obtained in this thesis work are presented in the following chapters:

In Chapter 2 and Chapter 3 together, an anatomical dataset (the Twente Spine Dataset) for musculoskeletal modeling of the entire human spine is presented. This anatomical dataset was obtained based on medical images and detailed dissection of one male cadaver and thus is the first complete and consistent atlas for the spine. In this dataset, the coordinates of muscle attachments, muscle morphological parameters, and skeletal geometry of the cadaver are reported. Moreover, new data are presented for several muscle groups.

Chapter 4 describes the development of the Twente Spine Model based on the dataset reported in Chapters 2 \& 3 . In this model, the individual vertebrae at the cervical, thoracic, and lumbar regions, a flexible ribcage, and a detailed muscle anatomy were represented. This full spine model essentially represents a subject-specific musculoskeletal system and, unlike generic models, does not require piecing anatomical data from other datasets. Inverse dynamics-based static optimization was employed for computing muscle and joint 
forces during physiological trunk and neck exertions. To validate the model's prediction of internal loads, intradiscal pressures at the lumbar and thoracic discs were estimated from predicted compressive forces, and these were compared to previous in vivo measurements. Furthermore, disc compressive forces were calculated during standing upright, flexion, extension, lateral bending, axial rotation of the spine and were presented as percentages of the total body weight.

Chapter 5 elaborates on the sensitivity of muscle and intervertebral disc force computations against potential errors in modeling muscle attachment sites (muscle origin, insertion, and via points). Each attachment location was perturbed in the Twente Spine Model (Chapter 4) during standing upright, flexion, lateral bending, and axial rotation of the trunk, and the changes at the T6/T7, T12/L1, and L4/L5 disc forces were calculated. In addition, an overall sensitivity index value was calculated for every perturbed muscle. This index is essentially a measure of the collective reaction of all muscles to maintain required joint moments due to perturbations. Certain muscle groups were identified as being more influential on predicted internal forces.

In Chapter 6 electromyographic activities of the major trunk and abdominal muscles and trunk movements were simultaneously measured in fourteen healthy individuals during isometric and dynamic trunk exertions. Segmental rotations were calculated at different spinal levels, and muscle activation trends were compared between the subject groups. Measured trunk tasks can be isometrically or dynamically simulated in inverse dynamics based spinal musculoskeletal models, and predicted and measured muscle activations can then be compared to justify musculoskeletal predictions.

In Chapter 7] musculoskeletal and patient-specific finite element models were pipelined to study if including physiological load cases instead of a simple loading regime can significantly affect predictions of the vertebral fracture risk. The Twente Spine Model presented in Chapter 4 was scaled by the body height and weight of a patient who had spinal metastasis to extract the boundary conditions at the L2/L3 joint during physiological trunk movements. These loading and boundary conditions were subsequently applied in a finite element model of the L2-L4 spinal unit for studying its mechanical response.

In Chapter 8, a general discussion on the key findings of this thesis work, as well as the current limitations and some future perspectives, are presented. 


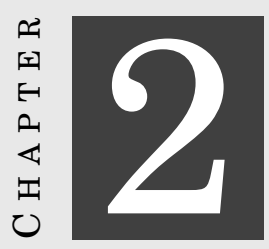

\title{
TWENTE SPINE MODEL: A COMPLETE AND COHERENT DATASET FOR MUSCULOSKELETAL MODELING OF THE LUMBAR REGION OF THE HUMAN SPINE
}

\begin{abstract}
Musculoskeletal modeling can greatly help in understanding normal and pathological functioning of the spine. For such models to produce reliable muscle and joint force estimations, an adequate set of musculoskeletal data is necessary. In this study, we present a complete and coherent dataset for the lumbar spine, based on medical images and dissection measurements from one embalmed human cadaver. We divided muscles into muscle-tendon elements, digitized their attachments at the bones and measured morphological parameters. In total, we measured 11 muscles from one body side, using 96 elements. For every muscle element, we measured three-dimensional coordinates of its attachments, fiber length, tendon length, sarcomere length, optimal fiber length, pennation angle, mass, and physiological cross-sectional area together with the geometry of the lumbar spine. Results were consistent with other anatomical studies and included new data for the serratus posterior inferior muscle. The dataset presented in this paper enables a complete and coherent musculoskeletal model for the lumbar spine and will improve the current state-of-the art in predicting spinal loading.
\end{abstract}

This chapter has been published as: Riza Bayoglu, Leo Geeraedts, Karlijn H. J. Groenen, Nico Verdonschot, Bart Koopman, Jasper Homminga, "Twente Spine Model: A complete and coherent dataset for musculoskeletal modeling of the lumbar region of the human spine”, Journal of Biomechanics, 2017, 53, 111-119. 


\subsection{Introduction}

The combination of flexibility and rigidity that characterizes the spine makes it vulnerable to a range of mechanical and medical problems, and it is therefore an important area for biomechanical research. Most spine research has focused on the lumbar spine with a special focus on low back pain [45-48] and disc herniation [49]. Despite high quality research on the lumbar spine, our understanding of its in vivo mechanical function remains limited due to its complex structure. Musculoskeletal models of the lumbar spine would enable clinical insights into its functioning, and allow answering what if questions [50].

The capability of a muscle to produce torques at the joints is directly related to its moment arms, and thus to its origin and insertion, and to its strength [35]. Therefore, representation of muscles in musculoskeletal models demands special care to simulate true anatomy and realistic in vivo muscle function. In the lower extremity, Carbone et al. (2012) demonstrated that small differences in muscle attachments may markedly affect muscle moment arms, and, thus affect muscle force predictions considerably [51]. Furthermore, morphological parameters such as optimal fiber length, tendon length and physiological cross-sectional area, are critical inputs into musculoskeletal models [52]. In short, accuracy of modeling lines-of-action and morphological parameters determine muscle activation levels and have significant effects on the output of a musculoskeletal model.

Currently there is no musculoskeletal dataset which enables the development of a complete and coherent model for the lumbar spine. None of the previous studies quantified muscle attachments at the bones and measured complete morphological parameters for all muscles of the lumbar spine [42, 53-58]. Instead, they typically focused on a smaller part of the lumbar spine and presented anatomical drawings to illustrate muscle attachments. Subsequently, current musculoskeletal models require piecing together data from these studies and making assumptions when data does not exist. Consequently, such models will require complex scaling between the geometries and the muscle architectures of the cadaveric specimens [26, 27, 34, 59]. This approach may then result in a musculoskeletal system that never really existed [38]. Therefore, a musculoskeletal model based on one complete and coherent dataset-obtained from one body-would further improve our understanding of the human spine and is, thus, a better approach for clinical use [60]. The aim of our work is to obtain a complete and coherent anatomical dataset for the entire human spine, but in this paper we focus on the lumbar spine alone. This dataset includes segmented bone surfaces, three-dimensional coordinates of muscle attachment sites, bony wrapping surfaces and morphological muscle parameters from a single human cadaver. 


\subsection{Materials and methods}

\subsubsection{Cadaveric specimen}

An embalmed human cadaver body (79 years-old, male, height: $154 \mathrm{~cm}$, mass: $51 \mathrm{~kg}$ ) was obtained with institutional approval from Radboud university medical center. The cause of death was Alzheimer. We noticed that the fifth lumbar vertebra was fused to the sacrum. In the cadaver, we distinguished 33 bones: twelve thoracic and four lumbar vertebrae, twelve ribs, sternum, humerus, femur, sacrum, and pelvis.

\subsubsection{Medical imaging}

Prior to dissection, we acquired full body supine computed tomography (CT) images (Siemens SOMATOM Sensation 64, Siemens AG, Munich, Germany, voxel size $0.977 \mathrm{~mm}$ x $0.977 \mathrm{~mm} \times 1 \mathrm{~mm})$.

\subsubsection{Cadaveric measurements}

We measured muscles of the lumbar spine from the right side of the trunk, therefore we removed complete skin and subcutaneous fat on this side. Subsequently, we inserted reference tube-screw connectors (see Fig. 2.1 a) in the bones under the guidance of a C-arm device (Phillips BV 29). These connectors were used to construct temporary local reference frames in the bones enabling the consistent alignment of the optical reference tool between position measurement sessions. To strengthen the fixation between the bones and the connectors, Polymethylmethacrylate (PMMA) was poured through the screw guide holes. The positions of muscle attachments in three-dimensions with respect to the corresponding reference frames of the bones were measured using the NDI Hybrid Polaris Spectra tracking system. Because it was not possible to insert the connectors in the ribs, muscles that had attachments to the ribs were measured with respect to other bones nearby (for example, an attachment site at the twelfth rib was measured with respect to the reference frame of the twelfth vertebra). A graphical user interface was developed in Matlab R2014b to communicate with the tracking system and was utilized to properly record the measurements. Prior to the experiment, we assessed the accuracy of using these connectors. We found a position error of $0.331 \pm 0.391 \mathrm{~mm}$ for measuring a single attachment point.

\subsubsection{Muscle attachment sites}

After identification of a target muscle, fat at the intramuscular connections was removed, and the muscle was divided into several muscle-tendon elements to represent its function more accurately in the musculoskeletal model. The number of elements (functionally different parts of a muscle) was decided by using a previously described method [61]. The dissections were performed by an experienced anatomist. After locating the tendons at 

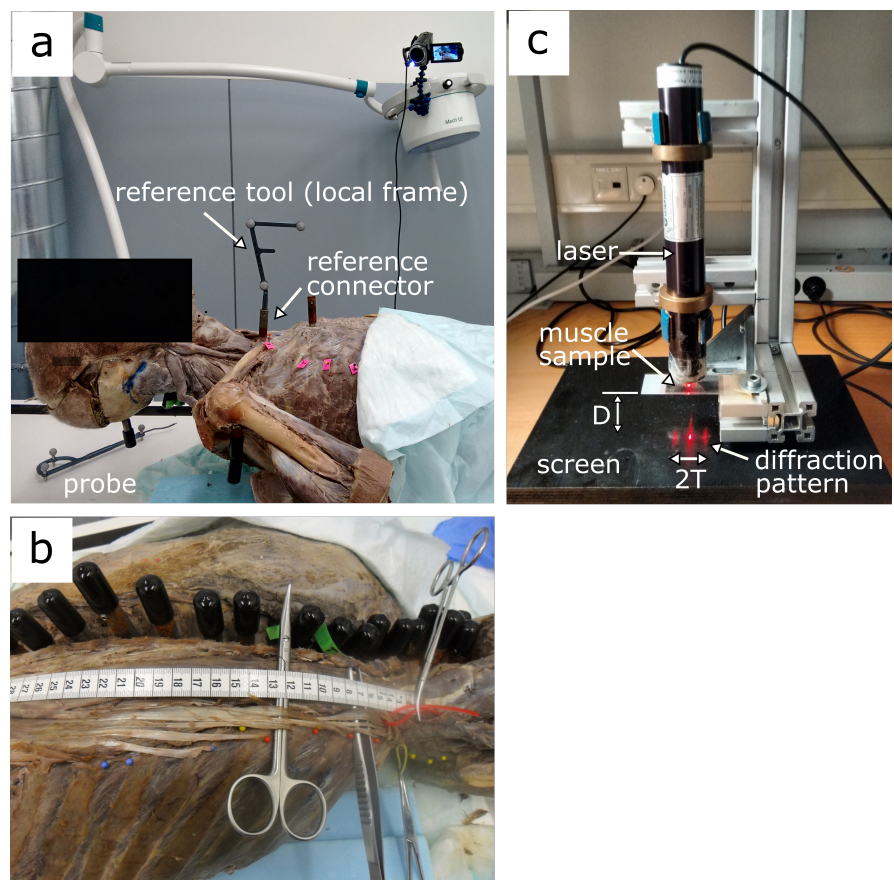

Figure 2.1: (a) An instance during position measurements. (b) Locating attachments of Erector Spinae muscle group. (c) Laser diffraction set-up used for sarcomere length measurements.

origin and insertion of the muscle elements, colored beads were used to mark the attachments of the elements (see Fig. 2.1p). Subsequently, we resected muscle elements and measured positions of their attachments at origin and insertion. We also measured the positions of via points (locations where a muscle is constrained to move) for elements with a curved lines-of-action. Depending on the size and shape of the muscles, we measured the attachments in one of the three geometrical forms, point, line or surface (see Fig. 2.2). Finally, we labeled the muscle elements and stored them in $2 \%$ formaldehyde solution until the measurement of morphological parameters.

The resection procedure differed slightly for iliocostalis, longissimus, and multifidus muscles. Dividing these muscles into elements in situ was not possible due to superficial and deep layers of muscle fibers. First, positions of attachments (where possible) at the bones were measured, and the entire muscle was resected afterwards. Later, they were micro-dissected and were divided into elements as done in previous anatomical studies [57, 62]. In addition, measuring the attachments of the psoas major muscle was impossible, as this muscle has its origins inside the abdominal cavity on the anterior side, which prevented the optical tools from being seen by the camera. We identified and noted its origins at the bones and later modeled their lines-of-action based on the study by Bogduk et al. (1992) [54]. 


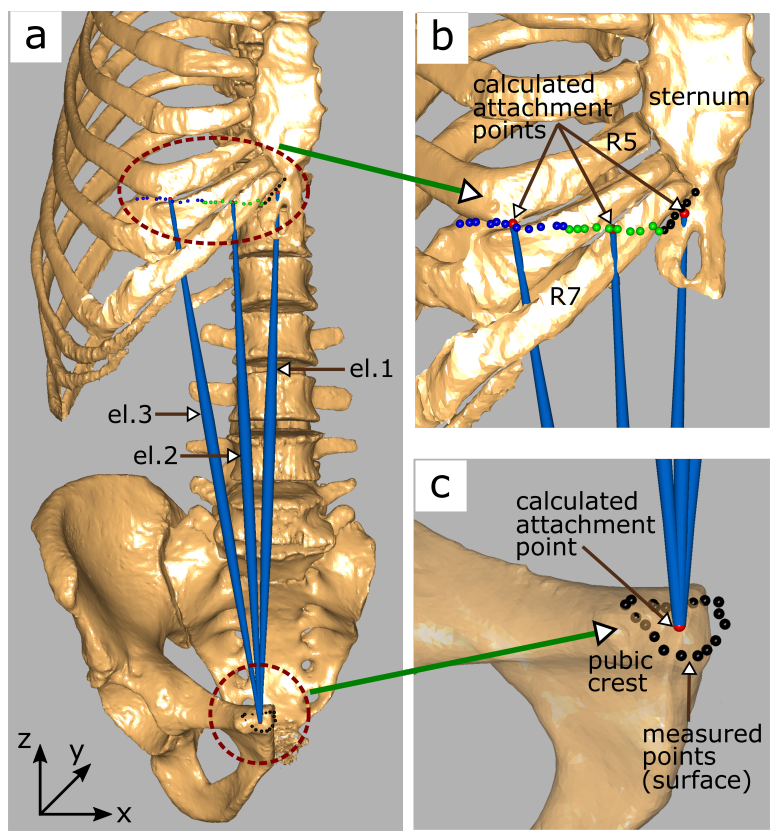

Figure 2.2: (a) Illustration of the geometrical forms used to classify line and surface-shaped muscle attachment sites. Rectus abdominis muscle is used for this illustration. (b) The insertions of this muscle was measured in line form. Black, green, and the blue dots represent the measured attachment points for the elements 1,2 , and 3 , respectively. Three red dots indicate calculated attachment points for these elements. Note that $n$ is equal to 1 in this case since the attachments of each element were measured separately. (c) Differently, the origin of the muscle was measured in surface form (black dots), and the red dot indicates the calculated attachment point (the centroid of the surface). Since this surface was considerably small, we opted to define one common origin for the all the elements $(\mathrm{n}=$ $1)$.

\subsubsection{Muscle morphological parameters}

For every element, we measured the following morphological muscle parameters: fiber length, tendon length, sarcomere length, optimal fiber length, pennation angle, mass, and physiological cross-sectional area. Prior to measuring these parameters, remaining fat and connective tissue on the surface of the muscle elements were carefully removed.

Pennation angle was measured by using a protractor (we neglected to measure the angles below $10^{\circ}$ ). Subsequently, the muscle element was placed on a flat table, and its musculotendon length (tendon-muscle-tendon length) between most proximal and distal ends was measured by using a ruler with a resolution of $0.5 \mathrm{~mm}$. Depending on the size of an element, we measured the lengths of up to six representative fibers to calculate an average fiber length for that element. Next, tendons were cut at both ends, and an average tendon length was calculated by subtracting average fiber length, multiplied by the cosine of the penna- 
tion angle, from measured musculo-tendon length. Then, we weighed the muscle elements (muscle fibers) by using a scale which had a resolution of $0.01 \mathrm{~g}$. Elements were wiped with tissue to remove excess fluid before weighing. Due to the complex tendinous connections of the abdomen muscles, obliquus externus abdominis and obliquus internus abdominis, we measured their tendon lengths while the muscles were still intact.

Average sarcomere length for every muscle was calculated by using the laser diffraction method [63]. For this, a vertical laser set-up which employed a Helium-Neon laser with a beam diameter of $2 \mathrm{~mm}$ and a wavelength of $632.8 \mathrm{~nm}$ was used (see Fig. 2.1 k). A constant distance $(D=55 \mathrm{~mm})$ was kept between the samples and reading screen throughout the measurements. We measured the distance between the first diffraction bands $(2 T$ in Fig. 2.1k) with a digital caliper (with a resolution of $0.01 \mathrm{~mm}$ ) and calculated sarcomere length (SL, in $\mu \mathrm{m}$ ) from this distance by using Eq. 2.1

$$
S L=\frac{632.8 \times 10^{-3} \times D \times \sqrt{\left(\frac{T}{D}\right)^{2}+1}}{T}
$$

Six to ten samples-consisting of one to five muscle fibers-were peeled from the representative element under an operation microscope and were used for the measurements. Samples were prepared from proximal end, distal end, and middle of the element. If the standard deviation of six samples exceeded $0.25 \mu \mathrm{m}$, we included up to four more samples taken between the proximal end and middle, and distal end and middle of the element. For each sample, we performed three sarcomere length measurements (at either ends and middle of the sample) and calculated the mean of three measurements. Subsequently, the average sarcomere length of a muscle was calculated from all the samples.

The optimal fiber length of an element (in $\mathrm{mm}$ ) was calculated by using Eq. 2.2.

$$
\ell_{o}^{f}=\frac{\ell^{f}}{\ell^{s}} \times 2.7
$$

where $\ell^{f}$ is the average fiber length and $\ell^{s}$ is the average sarcomere length of the muscle. We assumed an optimal sarcomere length of $2.7 \mu \mathrm{m}$ for skeletal muscles [61].

The volume of muscle elements was calculated by assuming a density of $1.0576 \mathrm{~g} / \mathrm{cm}^{3}$ for muscle tissue [61]. Moreover, physiological cross-sectional area (PCSA, in $\mathrm{cm}^{2}$ ) was calculated according to Eq. 2.3 .

$$
P C S A=\frac{m a s s \times \cos (\alpha)}{\rho \times \ell_{o}^{f}}
$$

where $\alpha$ is the pennation angle, $\rho$ is the muscle density, mass is the mass of the element, and $\ell_{o}^{f}$ is the optimal fiber length. In line with Brown et al. (2011), for the rectus abdominis PCSA was calculated as the largest measured regional PCSA and was distributed among its elements with respect to their masses [64]. Fiber lengths were summed for all regions. 


\subsubsection{Processing of muscle attachments}

We manually segmented CT images into stereolithography (STL) geometry files (Mimics 18.0 Materialise N. V., Leuven, Belgium). The iterative closest point algorithm was used to register point clouds (scanned over the bone surfaces) with the STL files [65]. See Appendix A.1 for more details. After registration, muscle attachments (measured with respect to the temporary local reference frames of the bones) were transformed to the global reference frame defined by the CT scanner. In the global reference frame, $x$-, $y$-, and $z$-axes point point laterally (to the left side of the cadaver), posteriorly, and cranially, respectively.

For line-shaped attachments, a polynomial of third degree was fit through the measured points. Subsequently, $n$ equidistant points ( $n$ is the number of elements that share an attachment site together) were calculated on the fitted polynomial curve as to represent the attachments of the elements [66]. The point-shaped attachments did not require further processing. For surface-shaped attachments, first a plane was fitted through the measured points. The measured points were projected onto the fitted plane, and a surface area was interpolated through the projected points. Later, the centroids of the $n$ equi-areal parts of the surface were calculated to represent the attachments for the elements [66], referring to [67]. Finally, all the calculated attachments were either projected on the surface of the STL files or left intact, depending on whether an element attached to only one bone or spanned between multiple bones, respectively.

Furthermore, we graphically estimated wrapping surfaces-from the geometry of the spine and measured point clouds over the structures that muscles wrap-in the AnyBody Modeling System $^{T M}$ version. 6.0.4 (AnyBody Technology A/S, Aalborg, Denmark). Depending on the shape of a surface, either a cylinder or an ellipsoid was estimated. See Appendix A.2 for details.

\subsection{Results}

The complete list of measured muscle elements is given in Table 2.3. In total, we measured 11 muscles from one side of the body using 96 muscle elements. For every element, we obtained the coordinates of its attachments at origin and insertion together with the morphological parameters: fiber length, sarcomere length, optimal fiber length, tendon length, pennation angle, mass, and physiological cross-sectional area (PCSA). Individual PCSAs were relatively small, the largest being $2.74 \mathrm{~cm}^{2}$ for an element of the psoas major. Total muscle PCSAs ranged from $2.07 \mathrm{~cm}^{2}$ for the serratus posterior inferior to $18.50 \mathrm{~cm}^{2}$ for the longissimus thoracis. Mean sarcomere lengths ranged from $2.14 \mu \mathrm{m}$ for the internal oblique to $3.57 \mu \mathrm{m}$ for the longissimus thoracis. Fiber length ranged from $2.0 \mathrm{~cm}$ for an element of the transversus abdominis to $26.3 \mathrm{~cm}$ for an element of the rectus abdominis. 
Mean optimal fiber lengths ranged from $4.0 \mathrm{~cm}$ for multifidus to $25.1 \mathrm{~cm}$ for the rectus abdominis. Tendon lengths for the thoracic component of the longissimus thoracis were very long, ranging from $7.4 \mathrm{~cm}$ to $26.6 \mathrm{~cm}$, while its lumbar component had relatively short tendon lengths. The definitions of wrapping surfaces can be found in Appendix A.2, and the coordinates of via points can be found in Appendix A.3 both as digital appendices. All the bones with re-constructed muscle lines-of-action were visualized in the AnyBody Modeling System $^{T M}$ ver. 6.0.4 (AnyBody Technology A/S, Aalborg, Denmark) and are depicted in Fig. 2.3
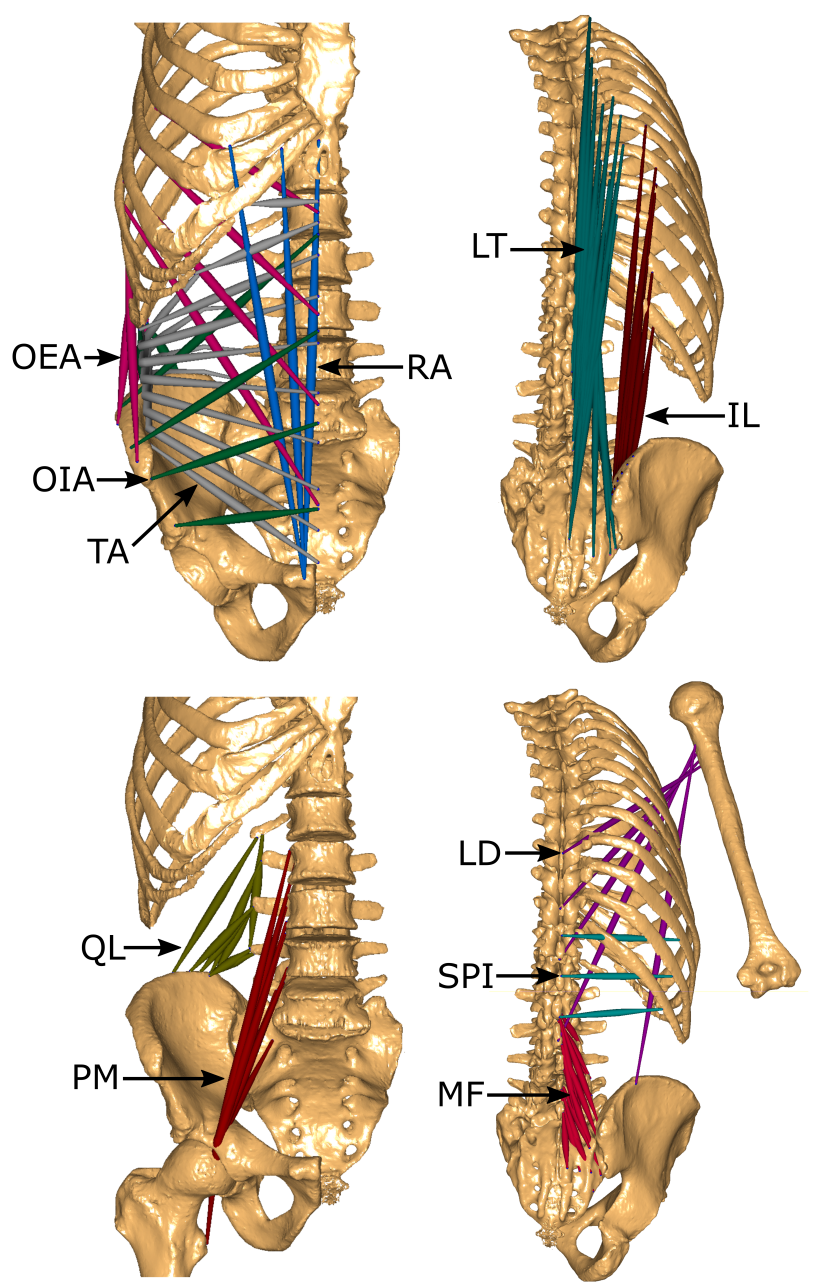

Figure 2.3: Dissected muscles in the musculoskeletal system. OEA: obliquus externus abdominis, OIA: obliquus internus abdominis, TA: transversus abdominis, RA: rectus abdominis, LT: longissimus thoracis, IL: iliocostalis lumborum, QL: quadratus lumborum, PM: psoas major, LD: latissimus dorsi, MF: multifidus, SPI: serratus posterior inferior. 
Table 2.1: Per muscle element: element number (\#), fiber length $\left(\ell^{f}\right)$, sarcomere length $\left(\ell^{s}\right)$, optimal fiber length $\left(\ell_{o}^{f}\right)$, tendon length $\left(\ell^{t}\right)$, pennation angle $(\alpha)$, physiological cross-sectional area (PCSA), and coordinates of attachments at origin and insertion with respect to the global reference frame defined by the CT scanner ${ }^{a, b, c}$.

\begin{tabular}{|c|c|c|c|c|c|c|c|c|c|c|c|c|c|c|c|c|c|c|}
\hline \multirow[t]{2}{*}{ Muscle } & \multirow[t]{2}{*}{ \# } & \multirow{2}{*}{$\begin{array}{l}\ell f \\
(\mathrm{~mm})\end{array}$} & \multirow{2}{*}{$\begin{array}{l}\ell^{s} \\
(\mu \mathrm{m})\end{array}$} & \multirow{2}{*}{$\begin{array}{l}\ell_{o}^{f} \\
(\mathrm{~mm})\end{array}$} & \multirow{2}{*}{$\begin{array}{l}\ell^{t} \\
(\mathrm{~mm})\end{array}$} & \multirow{2}{*}{$\begin{array}{l}\alpha \\
(\operatorname{deg})\end{array}$} & \multirow{2}{*}{$\begin{array}{l}\text { Mass } \\
\text { (g) }\end{array}$} & \multirow{2}{*}{$\begin{array}{l}\text { PCSA } \\
\left(\mathrm{cm}^{2}\right)\end{array}$} & \multirow{2}{*}{$\begin{array}{l}\text { Origin } \\
\text { (bone) }\end{array}$} & \multirow[t]{2}{*}{ Form } & \multicolumn{3}{|c|}{ Position (m) } & \multirow{2}{*}{$\begin{array}{l}\text { Insertion } \\
\text { (bone) }\end{array}$} & \multirow[t]{2}{*}{ Form } & \multicolumn{3}{|c|}{ Position (m) } \\
\hline & & & & & & & & & & & $\mathrm{x}$ & $\mathrm{y}$ & $\mathrm{z}$ & & & $\mathrm{x}$ & $\mathrm{y}$ & $\mathrm{z}$ \\
\hline Obliquus externus abdominis & 1 & 71.1 & 2.82 & 68.2 & 35.9 & 0 & 8.18 & 1.13 & Pelvis & Line & -0.1262 & -0.1046 & 0.7301 & R11 & Line & -0.1167 & -0.0965 & 0.7811 \\
\hline Obliquus externus abdominis & 2 & 103.8 & 2.82 & 99.6 & 95.2 & 0 & 19.04 & 1.81 & Pelvis & Line & -0.1365 & -0.1401 & 0.7092 & R10 & Surface & -0.1253 & -0.1010 & 0.8197 \\
\hline Obliquus externus abdominis & 3 & 125.6 & 2.82 & 120.4 & 172.4 & 0 & 20.29 & 1.59 & Pelvis & Line & -0.1233 & -0.1700 & 0.6836 & R9 & Surface & -0.1312 & -0.1159 & 0.8462 \\
\hline Obliquus externus abdominis & 4 & 131.7 & 2.82 & 126.2 & 153.4 & 0 & 16.59 & 1.24 & Linea alba & Line & 0.0000 & -0.2142 & 0.6541 & R8 & Surface & -0.1368 & -0.1413 & 0.8652 \\
\hline Obliquus externus abdominis & 5 & 96.7 & 2.82 & 92.7 & 120.7 & 0 & 12.76 & 1.30 & Linea alba & Line & 0.0000 & -0.2240 & 0.7226 & R7 & Line & -0.1324 & -0.1707 & 0.8752 \\
\hline Obliquus externus abdominis & 6 & 58.5 & 2.82 & 56.1 & 137.7 & 0 & 9.51 & 1.60 & Linea alba & Line & 0.0000 & -0.2316 & 0.7841 & R6 & Line & -0.1208 & -0.1893 & 0.8982 \\
\hline Obliquus externus abdominis & 7 & 56.2 & 2.82 & 53.9 & 83.7 & 0 & 1.53 & 0.27 & Linea alba & Line & 0.0000 & -0.2407 & 0.8538 & R5 & Line & -0.1109 & -0.1952 & 0.9254 \\
\hline Iliocostalis lumborum & 1 & 81.5 & 2.81 & 78.3 & 23.5 & 0 & 15.54 & 1.88 & Pelvis & Surface & -0.0601 & -0.0522 & 0.7270 & R11 & Point & -0.0776 & -0.0601 & 0.8403 \\
\hline Iliocostalis lumborum & 2 & 100.4 & 2.81 & 96.4 & 59.6 & 0 & 18.99 & 1.86 & Pelvis & Surface & -0.0551 & -0.0475 & 0.7198 & R10 & Point & -0.0766 & -0.0504 & 0.8878 \\
\hline Iliocostalis lumborum & 3 & 106.7 & 2.81 & 102.4 & 108.3 & 0 & 7.87 & 0.73 & Pelvis & Surface & -0.0503 & -0.0437 & 0.7122 & R9 & Point & -0.0741 & -0.0495 & 0.9221 \\
\hline Iliocostalis lumborum & 4 & 98.0 & 2.81 & 94.1 & 172.0 & 0 & 5.95 & 0.60 & Pelvis & Surface & -0.0466 & -0.0406 & 0.7057 & $\mathrm{R} 8$ & Point & -0.0800 & -0.0513 & 0.9646 \\
\hline Iliocostalis lumborum & 5 & 114.7 & 2.81 & 110.1 & 202.3 & 0 & 5.84 & 0.50 & Pelvis & Surface & -0.0440 & -0.0387 & 0.6989 & R7 & Point & -0.0798 & -0.0556 & 0.9789 \\
\hline Iliocostalis lumborum & 6 & 114.7 & 2.81 & 110.1 & 235.3 & 0 & 5.84 & 0.50 & Pelvis & Surface & -0.0420 & -0.0370 & 0.6941 & R6 & Point & -0.0709 & -0.0610 & 1.0160 \\
\hline Obliquus internus abdominis & 1 & 36.8 & 2.14 & 46.4 & 19.8 & 0 & 8.08 & 1.65 & Pelvis & Line & -0.0923 & -0.0777 & 0.7381 & R11 & Line & -0.1170 & -0.1012 & 0.7781 \\
\hline Obliquus internus abdominis & 2 & 55.5 & 2.14 & 70.1 & 29.5 & 0 & 13.23 & 1.78 & Pelvis & Line & -0.1194 & -0.0997 & 0.7329 & R10 & Line & -0.1216 & -0.1643 & 0.7738 \\
\hline Obliquus internus abdominis & 3 & 88.5 & 2.14 & 111.8 & 135.0 & 0 & 27.09 & 2.29 & Pelvis & Line & -0.1345 & -0.1294 & 0.7208 & Linea alba & Line & 0.0000 & -0.2365 & 0.8387 \\
\hline Obliquus internus abdominis & 4 & 57.9 & 2.14 & 73.1 & 117.1 & 0 & 8.92 & 1.15 & Pelvis & Line & -0.1275 & -0.1628 & 0.6931 & Linea alba & Line & 0.0000 & -0.2320 & 0.7729 \\
\hline Obliquus internus abdominis & 5 & 51.5 & 2.14 & 65.0 & 87.7 & 0 & 5.41 & 0.79 & Pelvis & Line & -0.1135 & -0.1783 & 0.6720 & Linea alba & Line & 0.0000 & -0.2237 & 0.7100 \\
\hline Obliquus internus abdominis & 6 & 56.5 & 2.14 & 71.4 & 64.5 & 0 & 4.83 & 0.64 & Pelvis & Line & -0.0973 & -0.1797 & 0.6399 & Linea alba & Line & 0.0000 & -0.2146 & 0.6513 \\
\hline Latissimus dorsi & 1 & 229.7 & 2.26 & 274.6 & 123.8 & 0 & 70.14 & 2.42 & Pelvis & Line & -0.0775 & -0.0647 & 0.7360 & Humerus & Line & -0.1382 & -0.1698 & 1.0543 \\
\hline Latissimus dorsi & 2 & 228.8 & 2.26 & 273.5 & 196.6 & 35 & 37.88 & 1.07 & $\mathrm{~L} 2$ & Line & -0.0034 & -0.0461 & 0.7770 & Humerus & Line & -0.1362 & -0.1652 & 1.0503 \\
\hline Latissimus dorsi & 3 & 209.7 & 2.26 & 250.7 & 120.3 & 0 & 37.62 & 1.42 & T11 & Line & -0.0046 & -0.0373 & 0.8546 & Humerus & Line & -0.1365 & -0.1616 & 1.0470 \\
\hline Latissimus dorsi & 4 & 190.5 & 2.26 & 227.7 & 112.0 & 0 & 19.27 & 0.80 & T9 & Line & -0.0055 & -0.0347 & 0.9029 & Humerus & Line & -0.1375 & -0.1588 & 1.0430 \\
\hline Latissimus dorsi & 5 & 171.3 & 2.26 & 204.8 & 103.7 & 0 & 9.74 & 0.45 & $\mathrm{~T} 7$ & Line & -0.0077 & -0.0364 & 0.9558 & Humerus & Line & -0.1385 & -0.1566 & 1.0400 \\
\hline Longissimus thoracis & 1 & 93.0 & 3.57 & 70.3 & 225.3 & 0 & 6.49 & 0.87 & L4 & Line & -0.0070 & -0.0475 & 0.7327 & R5 & Point & -0.0425 & -0.0749 & 1.0410 \\
\hline Longissimus thoracis & 2 & 80.7 & 3.57 & 61.0 & 215.8 & 0 & 0.77 & 0.12 & Sacrum & Line & -0.0073 & -0.0370 & 0.6866 & R8 & Point & -0.0403 & -0.0558 & 0.9749 \\
\hline Longissimus thoracis & 3 & 85.0 & 3.57 & 64.3 & 203.7 & 0 & 2.31 & 0.34 & Sacrum & Line & -0.0073 & -0.0370 & 0.6866 & $\mathrm{R} 8$ & Point & -0.0454 & -0.0496 & 0.9657 \\
\hline Longissimus thoracis & 4 & 105.3 & 3.57 & 79.6 & 217.7 & 0 & 2.51 & 0.30 & L5 & Line & -0.0081 & -0.0445 & 0.7140 & R6 & Point & -0.0492 & -0.0653 & 1.0247 \\
\hline
\end{tabular}

Continued on next page 
Table 2.1: Continued from previous page

\begin{tabular}{|c|c|c|c|c|c|c|c|c|c|c|c|c|c|c|c|c|c|c|}
\hline \multirow[t]{2}{*}{ Muscle } & \multirow[t]{2}{*}{ \# } & \multirow{2}{*}{$\begin{array}{l}\ell f \\
(\mathrm{~mm})\end{array}$} & \multirow{2}{*}{$\begin{array}{l}\ell^{s} \\
(\mu \mathrm{m})\end{array}$} & \multirow{2}{*}{$\begin{array}{l}\ell_{o}^{f} \\
(\mathrm{~mm})\end{array}$} & \multirow{2}{*}{$\begin{array}{l}\ell^{t} \\
(\mathrm{~mm})\end{array}$} & \multirow{2}{*}{$\begin{array}{l}\alpha \\
(\operatorname{deg})\end{array}$} & \multirow{2}{*}{$\begin{array}{l}\text { Mass } \\
\text { (g) }\end{array}$} & \multirow{2}{*}{$\begin{array}{l}\text { PCSA } \\
\left(\mathrm{cm}^{2}\right)\end{array}$} & \multirow{2}{*}{$\begin{array}{l}\text { Origin } \\
\text { (bone) }\end{array}$} & \multirow[t]{2}{*}{ Form } & \multicolumn{3}{|c|}{ Position (m) } & \multirow{2}{*}{$\begin{array}{l}\text { Insertion } \\
\text { (bone) }\end{array}$} & \multirow[t]{2}{*}{ Form } & \multicolumn{3}{|c|}{ Position (m) } \\
\hline & & & & & & & & & & & $\mathrm{x}$ & $\mathrm{y}$ & $\mathrm{z}$ & & & $\mathrm{x}$ & $\mathrm{y}$ & $\mathrm{z}$ \\
\hline Longissimus thoracis & 5 & 87.0 & 3.57 & 65.8 & 204.5 & 0 & 1.94 & 0.28 & Sacrum & Line & -0.0040 & -0.0270 & 0.6551 & R9 & Point & -0.0456 & -0.0473 & 0.9380 \\
\hline Longissimus thoracis & 6 & 77.0 & 3.57 & 58.2 & 240.4 & 0 & 1.05 & 0.17 & Sacrum & Line & -0.0073 & -0.0398 & 0.6919 & $\mathrm{R} 7$ & Point & -0.0513 & -0.0552 & 0.9990 \\
\hline Longissimus thoracis & 7 & 27.0 & 3.57 & 20.4 & 12.0 & 0 & 4.86 & 2.25 & Sacrum & Line & -0.0391 & -0.0559 & 0.7002 & L5 & Point & -0.0367 & -0.0690 & 0.7240 \\
\hline Longissimus thoracis & 8 & 42.0 & 3.57 & 31.8 & 18.0 & 0 & 5.69 & 1.69 & Pelvis & Line & -0.0406 & -0.0422 & 0.6979 & $\mathrm{~L} 4$ & Point & -0.0338 & -0.0784 & 0.7398 \\
\hline Longissimus thoracis & 9 & 64.0 & 3.57 & 48.4 & 20.0 & 0 & 7.90 & 1.54 & Pelvis & Line & -0.0412 & -0.0406 & 0.6962 & L3 & Point & -0.0263 & -0.0796 & 0.7652 \\
\hline Longissimus thoracis & 10 & 91.7 & 3.57 & 69.3 & 33.3 & 0 & 12.57 & 1.71 & Pelvis & Line & -0.0412 & -0.0406 & 0.6962 & $\mathrm{~L} 2$ & Point & -0.0247 & -0.0786 & 0.7876 \\
\hline Longissimus thoracis & 11 & 109.3 & 3.57 & 82.7 & 55.7 & 0 & 22.89 & 2.62 & Pelvis & Line & -0.0412 & -0.0406 & 0.6962 & $\mathrm{~L} 1$ & Point & -0.0240 & -0.0703 & 0.8157 \\
\hline Longissimus thoracis & 12 & 135.3 & 3.57 & 102.4 & 73.8 & 0 & 15.52 & 1.43 & Sacrum & Line & -0.0445 & -0.0426 & 0.6467 & $\mathrm{~T} 12$ & Point & -0.0220 & -0.0603 & 0.8448 \\
\hline Longissimus thoracis & 13 & 87.7 & 3.57 & 66.3 & 150.2 & 0 & 11.41 & 1.63 & Sacrum & Line & -0.0398 & -0.0397 & 0.6417 & T11 & Point & -0.0268 & -0.0529 & 0.8732 \\
\hline Longissimus thoracis & 14 & 102.8 & 3.57 & 77.7 & 168.6 & 0 & 4.08 & 0.50 & Sacrum & Line & -0.0253 & -0.0349 & 0.6400 & $\mathrm{~T} 10$ & Point & -0.0295 & -0.0476 & 0.9072 \\
\hline Longissimus thoracis & 15 & 63.5 & 3.57 & 48.0 & 216.3 & 0 & 1.43 & 0.28 & Sacrum & Line & -0.0040 & -0.0270 & 0.6551 & T9 & Point & -0.0299 & -0.0444 & 0.9292 \\
\hline Longissimus thoracis & 16 & 68.3 & 3.57 & 51.6 & 210.4 & 0 & 1.80 & 0.33 & Sacrum & Line & -0.0073 & -0.0370 & 0.6866 & T8 & Point & -0.0314 & -0.0481 & 0.9605 \\
\hline Longissimus thoracis & 17 & 57.8 & 3.57 & 43.7 & 241.2 & 0 & 1.18 & 0.26 & Sacrum & Line & -0.0073 & -0.0398 & 0.6919 & $\mathrm{~T} 7$ & Point & -0.0316 & -0.0531 & 0.9853 \\
\hline Longissimus thoracis & 18 & 82.3 & 3.57 & 62.3 & 224.7 & 0 & 2.43 & 0.37 & L5 & Line & -0.0081 & -0.0445 & 0.7140 & $\mathrm{~T} 6$ & Point & -0.0320 & -0.0618 & 1.0133 \\
\hline Longissimus thoracis & 19 & 76.3 & 3.57 & 57.7 & 237.6 & 0 & 2.54 & 0.42 & $\mathrm{~L} 4$ & Line & -0.0070 & -0.0475 & 0.7327 & T5 & Point & -0.0336 & -0.0752 & 1.0346 \\
\hline Longissimus thoracis & 20 & 67.0 & 3.57 & 50.7 & 266.3 & 0 & 1.40 & 0.26 & L4 & Line & -0.0076 & -0.0476 & 0.7410 & $\mathrm{~T} 4$ & Point & -0.0279 & -0.0896 & 1.0562 \\
\hline Longissimus thoracis & 21 & 119.8 & 3.57 & 90.6 & 223.3 & 0 & 3.83 & 0.40 & L3 & Line & -0.0086 & -0.0491 & 0.7572 & $\mathrm{~T} 3$ & Point & -0.0239 & -0.1049 & 1.0750 \\
\hline Longissimus thoracis & 22 & 126.0 & 3.57 & 95.3 & 223.6 & 0 & 6.06 & 0.60 & $\mathrm{~L} 2$ & Line & -0.0072 & -0.0481 & 0.7772 & $\mathrm{~T} 2$ & Point & -0.0209 & -0.1211 & 1.0978 \\
\hline Longissimus thoracis & 23 & 87.0 & 3.57 & 65.8 & 265.5 & 0 & 0.91 & 0.13 & L1 & Line & -0.0063 & -0.0463 & 0.8014 & $\mathrm{~T} 1$ & Point & -0.0222 & -0.1453 & 1.1100 \\
\hline Multifidus & 1 & 55.0 & 3.35 & 44.4 & 12.0 & 0 & 0.33 & 0.07 & Sacrum & Surface & -0.0121 & -0.0279 & 0.6555 & L5 & Point & -0.0082 & -0.0457 & 0.7081 \\
\hline Multifidus & 2 & 47.0 & 3.35 & 37.9 & 21.0 & 0 & 0.33 & 0.08 & Sacrum & Surface & -0.0348 & -0.0331 & 0.6513 & L5 & Point & -0.0100 & -0.0506 & 0.7048 \\
\hline Multifidus & 3 & 51.7 & 3.35 & 41.7 & 55.7 & 0 & 2.83 & 0.64 & Sacrum & Surface & -0.0368 & -0.0387 & 0.6333 & L4 & Point & -0.0065 & -0.0485 & 0.7303 \\
\hline Multifidus & 4 & 42.7 & 3.35 & 34.5 & 41.7 & 0 & 1.95 & 0.54 & Sacrum & Surface & -0.0241 & -0.0365 & 0.6528 & L4 & Point & -0.0061 & -0.0541 & 0.7279 \\
\hline Multifidus & 5 & 42.0 & 3.35 & 33.9 & 27.0 & 0 & 0.61 & 0.17 & Sacrum & Surface & -0.0334 & -0.0391 & 0.6747 & $\mathrm{~L} 4$ & Point & -0.0083 & -0.0612 & 0.7328 \\
\hline Multifidus & 6 & 38.6 & 3.35 & 31.1 & 72.8 & 0 & 4.37 & 1.33 & Sacrum & Surface & -0.0440 & -0.0416 & 0.6489 & L3 & Point & -0.0091 & -0.0511 & 0.7494 \\
\hline Multifidus & 7 & 34.1 & 3.35 & 27.5 & 28.3 & 0 & 1.93 & 0.66 & L5 & Surface & -0.0279 & -0.0533 & 0.6990 & L3 & Point & -0.0099 & -0.0580 & 0.7525 \\
\hline Multifidus & 8 & 27.5 & 3.35 & 22.2 & 19.5 & 0 & 0.71 & 0.30 & L5 & Point & -0.0350 & -0.0652 & 0.7250 & L3 & Point & -0.0092 & -0.0662 & 0.7530 \\
\hline Multifidus & 9 & 59.9 & 3.35 & 48.3 & 40.5 & 0 & 4.36 & 0.85 & Pelvis & Surface & -0.0407 & -0.0526 & 0.6850 & $\mathrm{~L} 2$ & Point & -0.0068 & -0.0496 & 0.7732 \\
\hline Multifidus & 10 & 57.7 & 3.35 & 46.6 & 30.7 & 0 & 4.17 & 0.85 & Sacrum & Surface & -0.0237 & -0.0518 & 0.6920 & $\mathrm{~L} 2$ & Point & -0.0068 & -0.0496 & 0.7732 \\
\hline Multifidus & 11 & 44.5 & 3.35 & 35.9 & 7.0 & 0 & 0.97 & 0.26 & L4 & Point & -0.0341 & -0.0799 & 0.7411 & $\mathrm{~L} 2$ & Point & -0.0089 & -0.0700 & 0.7759 \\
\hline Multifidus & 12 & 83.4 & 3.35 & 67.3 & 28.0 & 0 & 5.27 & 0.74 & Pelvis & Surface & -0.0417 & -0.0554 & 0.6988 & $\mathrm{~L} 1$ & Point & -0.0051 & -0.0496 & 0.7983 \\
\hline Multifidus & 13 & 72.7 & 3.35 & 58.6 & 33.8 & 0 & 4.74 & 0.76 & L5 & Surface & -0.0245 & -0.0546 & 0.6990 & L1 & Point & -0.0051 & -0.0486 & 0.7983 \\
\hline Multifidus & 14 & 57.3 & 3.35 & 46.3 & 4.7 & 0 & 2.37 & 0.48 & $\mathrm{~L} 4$ & Point & -0.0321 & -0.0748 & 0.7456 & $\mathrm{~L} 1$ & Point & -0.0036 & -0.0553 & 0.7965 \\
\hline Multifidus & 15 & 30.0 & 3.35 & 24.2 & 11.5 & 0 & 0.86 & 0.34 & L3 & Point & -0.0242 & -0.0838 & 0.7742 & $\mathrm{~L} 1$ & Point & -0.0064 & -0.0635 & 0.8023 \\
\hline Psoas major & 1 & 112.6 & 2.60 & 117.1 & 157.4 & 0 & 14.44 & 1.17 & Sacrum & Surface & -0.0356 & -0.0939 & 0.6951 & Femur & Line & -0.0818 & -0.1022 & 0.5526 \\
\hline
\end{tabular}

Continued on next page 
Table 2.1: Continued from previous page

\begin{tabular}{|c|c|c|c|c|c|c|c|c|c|c|c|c|c|c|c|c|c|c|}
\hline \multirow[t]{2}{*}{ Muscle } & \multirow[t]{2}{*}{ \# } & \multirow{2}{*}{$\begin{array}{l}\ell f \\
(\mathrm{~mm})\end{array}$} & \multirow{2}{*}{$\begin{array}{l}\ell^{s} \\
(\mu \mathrm{m})\end{array}$} & \multirow{2}{*}{$\begin{array}{l}\ell_{o}^{f} \\
(\mathrm{~mm})\end{array}$} & \multirow{2}{*}{$\begin{array}{l}\ell^{t} \\
(\mathrm{~mm})\end{array}$} & \multirow{2}{*}{$\begin{array}{l}\alpha \\
(\operatorname{deg})\end{array}$} & \multirow{2}{*}{$\begin{array}{l}\text { Mass } \\
\text { (g) }\end{array}$} & \multirow{2}{*}{$\begin{array}{l}\text { PCSA } \\
\left(\mathrm{cm}^{2}\right)\end{array}$} & \multirow{2}{*}{$\begin{array}{l}\text { Origin } \\
\text { (bone) }\end{array}$} & \multirow[t]{2}{*}{ Form } & \multicolumn{3}{|c|}{ Position (m) } & \multirow{2}{*}{$\begin{array}{l}\text { Insertion } \\
\text { (bone) }\end{array}$} & \multirow[t]{2}{*}{ Form } & \multicolumn{3}{|c|}{ Position (m) } \\
\hline & & & & & & & & & & & $\mathrm{x}$ & $\mathrm{y}$ & $\mathrm{z}$ & & & $\mathrm{x}$ & $\mathrm{y}$ & $\mathrm{z}$ \\
\hline Psoas major & 2 & 112.8 & 2.60 & 117.2 & 223.8 & 0 & 21.13 & 1.70 & L3L4IVD & Surface & -0.0280 & -0.1080 & 0.7360 & Femur & Line & -0.0818 & -0.1022 & 0.5526 \\
\hline Psoas major & 3 & 138.0 & 2.60 & 143.4 & 201.0 & 0 & 11.84 & 0.78 & L3 & Surface & -0.0336 & -0.0922 & 0.7600 & Femur & Line & -0.0818 & -0.1022 & 0.5526 \\
\hline Psoas major & 4 & 97.5 & 2.60 & 101.3 & 200.5 & 0 & 4.68 & 0.44 & L4 & Surface & -0.0329 & -0.0960 & 0.7301 & Femur & Line & -0.0818 & -0.1022 & 0.5526 \\
\hline Psoas major & 5 & 115.8 & 2.60 & 120.4 & 223.2 & 0 & 14.63 & 1.15 & $\mathrm{~L} 2$ & Surface & -0.0260 & -0.0909 & 0.7890 & Femur & Line & -0.0818 & -0.1022 & 0.5526 \\
\hline Psoas major & 6 & 126.6 & 2.60 & 131.6 & 226.9 & 0 & 38.08 & 2.74 & L1 & Surface & -0.0192 & -0.0897 & 0.8199 & Femur & Line & -0.0818 & -0.1022 & 0.5526 \\
\hline Psoas major & 7 & 128.4 & 2.60 & 133.4 & 206.6 & 0 & 31.76 & 2.25 & L1 & Surface & -0.0231 & -0.0984 & 0.8289 & Femur & Line & -0.0818 & -0.1022 & 0.5526 \\
\hline Psoas major & 8 & 108.0 & 2.60 & 112.2 & 191.5 & 0 & 13.56 & 1.14 & L3 & Surface & -0.0248 & -0.1085 & 0.7462 & Femur & Line & -0.0818 & -0.1022 & 0.5526 \\
\hline Psoas major & 9 & 131.3 & 2.60 & 136.5 & 206.7 & 0 & 11.75 & 0.81 & L2L3IVD & Surface & -0.0270 & -0.1150 & 0.7700 & Femur & Line & -0.0818 & -0.1022 & 0.5526 \\
\hline Psoas major & 10 & 136.3 & 2.60 & 141.7 & 211.7 & 0 & 12.09 & 0.81 & L2 & Surface & -0.0257 & -0.1140 & 0.7784 & Femur & Line & -0.0818 & -0.1022 & 0.5526 \\
\hline Quadratus lumborum & 1 & 21.0 & 2.84 & 20.0 & 31.0 & 0 & 2.48 & 1.17 & Pelvis & Surface & -0.0791 & -0.0783 & 0.7431 & L3 & Line & -0.0488 & -0.0827 & 0.7563 \\
\hline Quadratus lumborum & 2 & 26.2 & 2.84 & 24.9 & 29.8 & 0 & 0.89 & 0.34 & Pelvis & Surface & -0.0755 & -0.0713 & 0.7428 & $\mathrm{~L} 2$ & Line & -0.0493 & -0.0791 & 0.7892 \\
\hline Quadratus lumborum & 3 & 37.4 & 2.84 & 35.6 & 33.6 & 0 & 4.00 & 1.06 & Pelvis & Line & -0.0859 & -0.0756 & 0.7434 & $\mathrm{~L} 2$ & Line & -0.0491 & -0.0799 & 0.7890 \\
\hline Quadratus lumborum & 4 & 60.7 & 2.84 & 57.8 & 35.3 & 0 & 3.06 & 0.50 & Pelvis & Line & -0.0785 & -0.0697 & 0.7427 & L1 & Point & -0.0433 & -0.0757 & 0.8211 \\
\hline Quadratus lumborum & 5 & 51.4 & 2.84 & 48.9 & 32.1 & 0 & 4.85 & 0.94 & L3 & Point & -0.0513 & -0.0816 & 0.7607 & R12 & Line & -0.0425 & -0.0700 & 0.8398 \\
\hline Quadratus lumborum & 6 & 26.3 & 2.84 & 25.1 & 29.7 & 0 & 1.99 & 0.75 & Pelvis & Line & -0.0798 & -0.0810 & 0.7410 & L3 & Point & -0.0513 & -0.0825 & 0.7589 \\
\hline Quadratus lumborum & 7 & 33.0 & 2.84 & 31.4 & 25.5 & 0 & 3.07 & 0.92 & Pelvis & Line & -0.0932 & -0.0875 & 0.7439 & $\mathrm{~L} 2$ & Line & -0.0491 & -0.0799 & 0.7890 \\
\hline Quadratus lumborum & 8 & 52.0 & 2.84 & 49.5 & 64.0 & 0 & 7.81 & 1.49 & Pelvis & Line & -0.1052 & -0.0925 & 0.7430 & R12 & Line & -0.0466 & -0.0702 & 0.8375 \\
\hline Rectus abdominis & 1 & 262.7 & 2.72 & 260.3 & 50.3 & 0 & 28.23 & 1.13 & Pelvis & Surface & -0.0094 & -0.1766 & 0.6040 & Sternum & Line & 0.0005 & -0.2333 & 0.9028 \\
\hline Rectus abdominis & 2 & 253.3 & 2.72 & 251.0 & 54.2 & 0 & 56.19 & 2.24 & Pelvis & Surface & -0.0094 & -0.1766 & 0.6040 & R7 & Line & -0.0249 & -0.2437 & 0.8972 \\
\hline Rectus abdominis & 3 & 244.3 & 2.72 & 242.1 & 66.7 & 0 & 31.53 & 1.26 & Pelvis & Surface & -0.0094 & -0.1766 & 0.6040 & R5 & Line & -0.0602 & -0.2448 & 0.8992 \\
\hline Serratus posterior inferior & 1 & 27.1 & 2.74 & 26.7 & 98.4 & 0 & 1.80 & 0.64 & L1 & Line & -0.0047 & -0.0447 & 0.7996 & R11 & Line & -0.1026 & -0.0774 & 0.8072 \\
\hline Serratus posterior inferior & 2 & 43.2 & 2.74 & 42.5 & 89.8 & 0 & 3.39 & 0.75 & $\mathrm{~T} 12$ & Line & -0.0064 & -0.0397 & 0.8387 & R10 & Line & -0.1119 & -0.0818 & 0.8384 \\
\hline Serratus posterior inferior & 3 & 53.3 & 2.74 & 52.4 & 98.3 & 0 & 3.79 & 0.68 & $\mathrm{~T} 10$ & Line & -0.0071 & -0.0360 & 0.8774 & R9 & Line & -0.1187 & -0.0907 & 0.8732 \\
\hline Transversus abdominus & 1 & 47.5 & 2.83 & 45.2 & 69.0 & 0 & 1.36 & 0.28 & Pelvis & Line & -0.1146 & -0.1588 & 0.6950 & Linea alba & Line & 0.0000 & -0.2005 & 0.6147 \\
\hline Transversus abdominus & 2 & 56.5 & 2.83 & 53.8 & 89.5 & 0 & 1.36 & 0.24 & Pelvis & Line & -0.1157 & -0.1100 & 0.7359 & Linea alba & Line & 0.0000 & -0.2086 & 0.6368 \\
\hline Transversus abdominus & 3 & 112.0 & 2.83 & 106.7 & 159.8 & 10 & 2.91 & 0.25 & $\mathrm{~L} 4$ & Line & -0.0484 & -0.0828 & 0.7386 & Linea alba & Line & 0.0000 & -0.2147 & 0.6648 \\
\hline Transversus abdominus & 4 & 115.0 & 2.83 & 109.5 & 157.3 & 10 & 2.91 & 0.25 & L3 & Line & -0.0454 & -0.0808 & 0.7590 & Linea alba & Line & 0.0000 & -0.2192 & 0.6967 \\
\hline Transversus abdominus & 5 & 115.0 & 2.83 & 109.5 & 161.1 & 10 & 2.91 & 0.25 & $\mathrm{~L} 2$ & Line & -0.0463 & -0.0787 & 0.7900 & Linea alba & Line & 0.0000 & -0.2225 & 0.7306 \\
\hline Transversus abdominus & 6 & 105.0 & 2.83 & 100.0 & 168.7 & 10 & 2.91 & 0.27 & L1 & Line & -0.0396 & -0.0745 & 0.8240 & Linea alba & Line & 0.0000 & -0.2251 & 0.7646 \\
\hline Transversus abdominus & 7 & 50.0 & 2.83 & 47.6 & 65.8 & 10 & 1.96 & 0.38 & R11 & Line & -0.1195 & -0.1054 & 0.7761 & Linea alba & Line & 0.0000 & -0.2276 & 0.7966 \\
\hline Transversus abdominus & 8 & 49.0 & 2.83 & 46.7 & 43.6 & 10 & 1.96 & 0.39 & R10 & Line & -0.1216 & -0.1643 & 0.7738 & Linea alba & Line & 0.0000 & -0.2303 & 0.8247 \\
\hline Transversus abdominus & 9 & 35.0 & 2.83 & 33.3 & 24.7 & 10 & 1.96 & 0.55 & R9 & Line & -0.0839 & -0.2217 & 0.8121 & Linea alba & Line & 0.0000 & -0.2338 & 0.8469 \\
\hline
\end{tabular}

Continued on next page

\section{$N$}




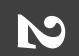

Table 2.1: Continued from previous page

\begin{tabular}{|c|c|c|c|c|c|c|c|c|c|c|c|c|c|c|c|c|c|c|}
\hline \multirow[t]{2}{*}{ Muscle } & \multirow[t]{2}{*}{$\#$} & \multirow{2}{*}{$\begin{array}{l}\ell f \\
(\mathrm{~mm})\end{array}$} & \multirow{2}{*}{$\begin{array}{l}\ell^{s} \\
(\mu \mathrm{m})\end{array}$} & \multirow{2}{*}{$\begin{array}{l}\ell_{o}^{f} \\
(\mathrm{~mm})\end{array}$} & \multirow{2}{*}{$\begin{array}{l}\ell^{t} \\
(\mathrm{~mm})\end{array}$} & \multirow{2}{*}{$\begin{array}{l}\alpha \\
(\operatorname{deg})\end{array}$} & \multirow{2}{*}{$\begin{array}{l}\text { Mass } \\
\text { (g) }\end{array}$} & \multirow{2}{*}{$\begin{array}{l}\text { PCSA } \\
\left(\mathrm{cm}^{2}\right)\end{array}$} & \multirow{2}{*}{$\begin{array}{l}\text { Origin } \\
\text { (bone) }\end{array}$} & \multirow[t]{2}{*}{ Form } & \multicolumn{3}{|c|}{ Position (m) } & \multirow{2}{*}{$\begin{array}{l}\text { Insertion } \\
\text { (bone) }\end{array}$} & \multirow[t]{2}{*}{ Form } & \multicolumn{3}{|c|}{ Position (m) } \\
\hline & & & & & & & & & & & $\mathrm{x}$ & $\mathrm{y}$ & $\mathrm{z}$ & & & $\mathrm{x}$ & $\mathrm{y}$ & $\mathrm{z}$ \\
\hline Transversus abdominus & 10 & 20.0 & 2.83 & 19.0 & 27.1 & 10 & 1.96 & 0.96 & R7 & Line & -0.0464 & -0.2393 & 0.8595 & Linea alba & Line & 0.0000 & -0.2385 & 0.8614 \\
\hline
\end{tabular}

${ }^{\mathrm{a}}$ Musculo-tendon lengths of longissimus thoracis elements were estimated based on their modeled lines-of-action.

${ }^{b}$ Unfortunately, we accidentally removed the superficial fibers of multifidus around the pelvis. The presented morphological parameters for these elements were estimated based on their modeled lines-of-action.

${ }^{c}$ The mathematical definitions of wrapping surfaces for the psoas major, latissimus dorsi, serratus posterior inferior, obliquus externus abdominis, obliquus internus abdominis, rectus abdominis, and transversus abdominis muscles are presented in Appendix A.2, and the via points for the psoas major, longissimus thoracis, iliocostalis lumborum, and the transversus abdominis muscles are given in Appendix A.3 


\subsection{Discussion}

In this study we measured a musculoskeletal dataset for the lumbar region of the trunk, consisting of the coordinates of muscle attachments, three-dimensional geometry of the bones (in the form of STL files), and morphological parameters of muscles. We noticed that most muscles had curved lines-of-action. Therefore, the coordinates of via points and the definitions of wrapping surfaces were provided in addition to their origins and insertions. For every muscle element, we provided morphological parameters: fiber length, tendon length, sarcomere length, optimal fiber length, pennation angle, mass, and PCSA. These parameters facilitate better simulation of muscle mechanics and hence will improve these types of models [74]. As the dataset was obtained from a single cadaver, it is complete and coherent and omits the uncertainties associated with combining musculoskeletal data from different specimens and measurement methods [75, 76]. Additionally, it includes new data for the serratus posterior inferior muscle. As such, the dataset enables construction of a complete and coherent musculoskeletal model for the lumbar spine. With this dataset we aim to contribute to an improvement of the state-of-the art in predicting lumbar spinal loading. This dataset is freely available to be used for non-commercial purposes through https://www.utwente.nl/en/et/bw/research/projects/twentespinemodel, upon the acceptance of our research license agreement.

During measurements we observed some interesting differences compared to previous studies. Firstly, we did not encounter the lumbar fascicles of iliocostalis lumborum as seen by Macintosh et al. (1987) [62], but encountered the lumbar fascicles of longissimus thoracis. Our findings were thus more in line with those of [77] who did not find any attachment of the iliocostalis to the lumbar transverse processes. Secondly, the thoracic component of the longissimus thoracis only had attachments at ribs 5-9, whereas [62] found connections at ribs 6-12. Thirdly, we did not find any bundles of psoas major originating from the L1L2 disc nor from the L4L5 disc, but we observed bundles originating from the L2 and L3 vertebrae. Small variations were also found for other muscles. These disparities are most likely explained by the variations in the anatomy of the specimens and the differences in the techniques.

This research had several limitations. Firstly, we noticed a slight scoliosis around the cadaver's neck, and after dissection of the muscles we discovered that L5 vertebra was fused to the sacrum [78]. One would not expect an effect on the muscle architecture in the lumbar region due to the scoliosis, however the same may not be true due to the sacralization. The muscles between the L5 and sacrum are thus most likely different in this cadaver. Secondly, we dissected only on the right side of the specimen. When a model is built upon this dataset, this requires assumptions about the skeletal geometry and the muscle architecture on the left side. Although it is simply convenient to build a left-right symmetrical model, no one 


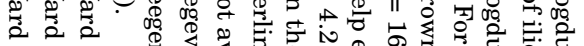

o o o

உ थ ? थ थ

管爱爱

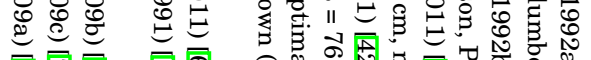

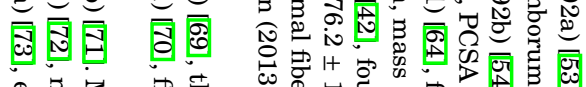

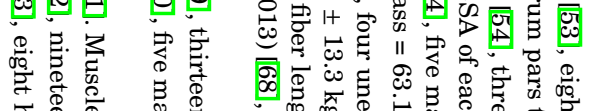

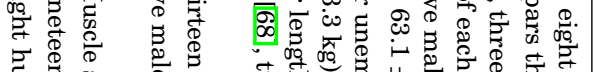

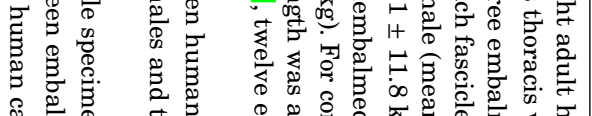

墨

政

욜.

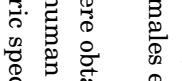

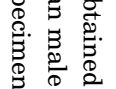

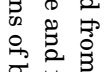

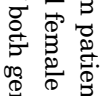

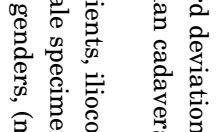

政

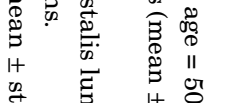

:

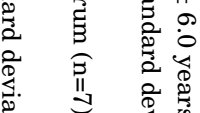

4

蛋.

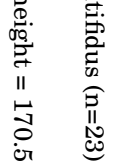

I+

$\stackrel{\infty}{*}$

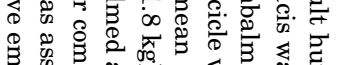

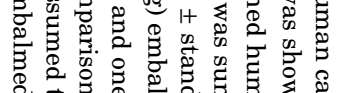

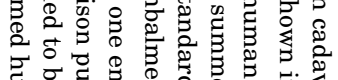

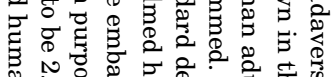

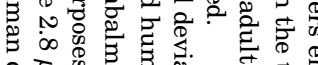

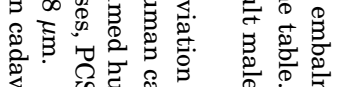

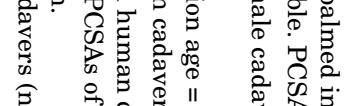

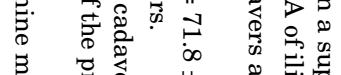

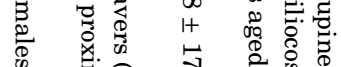

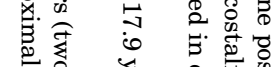

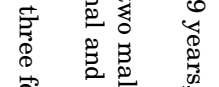

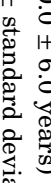

象 迎

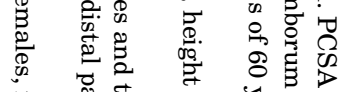

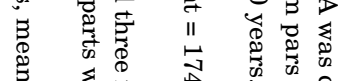

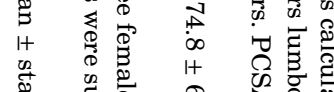

है दै की

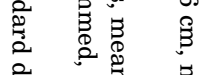

औ

की 3 व

कृ

टृ.

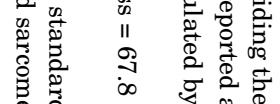

$\begin{array}{llllll}0 & 0 & 1+ & 2 & 0 & 0 \\ 0 & 0 & 0 & 0 \\ 0 & 0 & 0 & 0 \\ 0 & 0 & 0 & 0\end{array}$

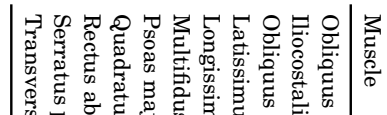

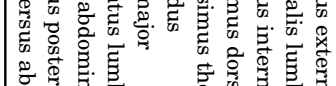

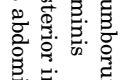

胥

क्ष

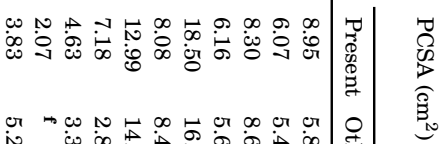

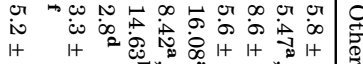

0 O

강

के

$\begin{array}{lllll}1+ & 1+ & 0 & \$ & 0 \\ 0 & + & 0 & 0\end{array}$

io

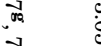

감

ind

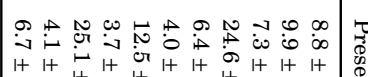

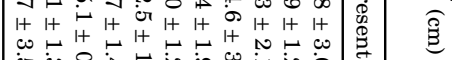

or co 0 is is i

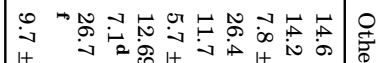

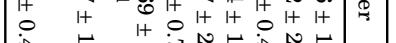

ब क

in

$\begin{array}{ll}\infty & 5 \\ 0 & 5 \\ 1+ & 8\end{array}$

io $1+$

के

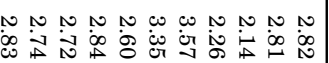

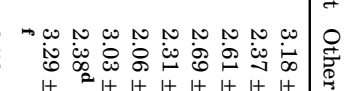

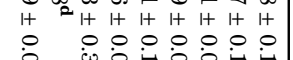

- क्ष

م

$\begin{array}{cccc}1+ & 1+ & 1+ & 1+ \\ 0 & 0 & 0 & 0\end{array}$

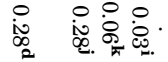

$\dot{0}$
$\dot{0}$
$1+$
0
$\dot{\circ}$
$\dot{A}$.

․ㅠ.

要.

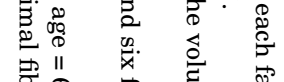

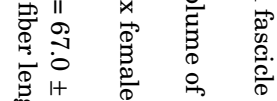

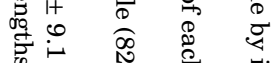

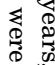

赵

品

ir

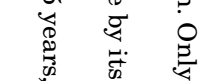


ever has a perfectly symmetrical musculoskeletal system. Thirdly, measured morphological parameters may not represent in vivo function accurately. Cutts et al. (1988) reported no significant decrease in muscle fiber lengths when the muscles were fixed on the skeleton, but a mean shrinkage of $2 \%$ when the muscles were fixed in isolation [79]. This implies that sarcomere and fiber lengths measured in-vitro may be slightly lower than in vivo. Similarly, measured optimal fiber lengths and physiological cross-sectional areas may change slightly. Fourthly, this dataset was obtained from an elderly male and thus represents a subject specific model, not a generic one. Therefore, certain care should be allocated if a personalized model is aimed to be built upon this dataset. Fifthly, we represented the mechanical function of large muscles by dividing them into a number of muscle-tendon elements. This method simplified the complex function of skeletal muscles and made it suitable to implement in musculoskeletal models. It is important to emphasize that this approach was a modelling procedure in itself and required some assumptions. The details and underlying assumptions of this method were previously described [80].

Laser diffraction is an accurate method for measuring sarcomere length in skeletal muscle fibers [63]. We used this method to calculate an average sarcomere length for every muscle and hence to estimate optimal fiber lengths, and PCSAs of its elements. Measured mean sarcomere lengths varied from $2.14 \mu \mathrm{m}$ to $3.57 \mu \mathrm{m}$, which is consistent with the data from Walker et al. (1974) [81]. Overall mean and standard deviation on the mean sarcomere lengths were $2.79 \pm 0.41 \mu \mathrm{m}$, implying that muscles measured in this study were close to their optimal lengths $(\sim 2.7 \mu \mathrm{m})$. There exists evidence that the distribution of sarcomere lengths within a muscle fiber is not uniform [82]. Previous studies elaborated on the needed sample size to effectively calculate a mean sarcomere length for a muscle and the effect of sample size on optimal fiber length variation. Langenderfer et al. (2004) showed for the shoulder and elbow muscles that the standard deviation of mean optimal muscle length was about $1.25 \mathrm{~mm}$ for 120 samples and increased to nearly $4 \mathrm{~mm}$ for 10 samples [83]. Our measurements also confirmed the non-uniform nature of sarcomere length distributions within the muscle fibers. We performed 18 sarcomere length measurements $(6$ samples $\times 3$ measurements). Therefore, the optimal fiber lengths and PCSAs presented in this paper should be regarded with similar variation. Furthermore, we compared morphological parameters such as PCSAs, optimal fiber lengths, and sarcomere lengths, measured in this study with similar anatomical studies in Table 2.2. The data reported in literature indicates some variations for these parameters, especially for PCSAs of the longissimus thoracis, latissimus dorsi, multifidus, and psoas major muscles. Although, in general, we found similar sarcomere lengths with the other studies, we systematically calculated relatively shorter optimal fiber lengths in the present study. The comparison of the fiber lengths revealed lower fiber lengths compared to the other studies. This was attributed to the differences between the heights of the cadavers measured $(154 \mathrm{~cm}$ in this study and around $170 \mathrm{~cm}$ in others). Other reasons which led to such differences may be due to differences 
in the measurement techniques and the number of samples used for sarcomere length measurements. Moreover, our PCSAs fit with the range of data reported in literature.

In the last decade, the demand for individualized musculoskeletal models has considerably increased aiming to improve the quality of patient specific treatment options. The state-of-the-art approach to obtain personalized models is to morph the medical images of a person to a previously built atlas containing muscle tendon attachment sites and lines-of-action [66, 76]. However, employing such an approach to create personalized models of the spine may only be reasonable up to some degree due to the high variability of muscle attachments with the bones. In this sense, imaging modalities should be improved to help more with creation of such models enabling identification of muscle attachments and obtaining muscle architectural parameters from living subjects [33, 50]. This objective is definitely one of the future challenges in musculoskeletal modeling. Meanwhile, we hope that the dataset reported hereby will be a great value to researchers in the field.

\section{Conflict of interest}

None of the authors have any financial or personal relationships with other people or organization that could inappropriately influence their work.

\section{Acknowledgments}

We gratefully acknowledge the financial supports by Fonds NutsOhra and the European Research Council 'the BiomechTools project'. We also like to thank several colleagues for their help on this research, Vincenzo Carbone and Kenan Niu for great discussions on musculoskeletal modeling and working with motion capture systems, Frans Segerink for providing a laser for sarcomere length measurements, Andre Sprengers for his help during CT and MRI scans, and Radiology Department and the Anatomy Department of the Radboud university medical center for their hospitality during the medical imaging and cadaveric measurement sessions. Furthermore, we thank Huub Maas from VU University of Amsterdam for his advice on laser diffraction method. 


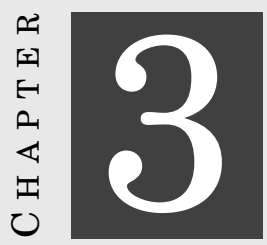

TWENTE SPINE MODEL: A COMPLETE AND COHERENT DATASET FOR MUSCULOSKELETAL MODELING OF THE THORACIC AND CERVICAL REGIONS OF THE HUMAN

\begin{abstract}
Musculoskeletal modeling could play a key role in advancing our understanding of the healthy and pathological spine, but the credibility of such models are strictly dependent on the accuracy of the anatomical data incorporated. In this study, we present a complete and coherent musculoskeletal dataset for the thoracic and cervical regions of the human spine, obtained through detailed dissection of an embalmed male cadaver. We divided the muscles into a number of muscle-tendon elements, digitized their attachments at the bones, and measured morphological muscle parameters. In total, 225 muscle elements were measured over 39 muscles. For every muscle element, we provide the coordinates of its attachments, fiber length, tendon length, sarcomere length, optimal fiber length, pennation angle, mass, and physiological cross-sectional area together with the skeletal geometry of the cadaver. Results were consistent with similar anatomical studies. Furthermore, we report new data for several muscles such as rotatores, multifidus, levatores costarum, spinalis, semispinalis, subcostales, transversus thoracis, and intercostales muscles. This dataset complements our previous study where we presented a consistent dataset for the lumbar region of the spine [84]. Therefore, when used together, these datasets enable a complete and coherent dataset for the entire spine. The complete dataset will be used to develop a musculoskeletal model for the entire human spine to study clinical and ergonomic applications.
\end{abstract}

This chapter has been published as: Riza Bayoglu, Leo Geeraedts, Karlijn H. J. Groenen, Nico Verdonschot, Bart Koopman, Jasper Homminga, "Twente Spine Model: A complete and coherent dataset for musculoskeletal modeling of the thoracic and cervical regions of the human spine”, Journal of Biomechanics, 2017, 58, 52-63. 


\subsection{Introduction}

Musculoskeletal models provide clinically useful information for understanding the normal and pathological functioning of the spine [24, 59]. Once validated, such models are valuable tools which can optimize surgical interventions and improve current treatment techniques [26, 33, 34, 85]. For example, the effect of posture on spinal loads and muscle forces can be explored [86] or the effect of resection of muscles on the activity of other muscles can be studied before a spinal surgery so that an effective approach is planned [17].

Previous anatomical studies on the spine mainly focused on the cervical and lumbar regions. Kamibayashi et al. (1998) [52] measured the morphological parameters of some neck muscles from several cadavers, and Borst et al. (2011) [38] measured muscle attachment sites and the morphological parameters of all neck muscles from a single cadaver. Other studies dissected muscles from the lumbar spine, presented anatomical drawings to illustrate muscle attachments, and measured the morphological parameters [42, 53-[58]. To the knowledge of the authors, there is, however, no anatomical dataset which enables developing a complete and coherent musculoskeletal model for the entire human spine. The lack of such coherent musculoskeletal data requires current models to combine data from several cadavers. This approach then necessitates anatomical scaling between the skeletal geometries of the spines and the muscle architectures of the cadavers. As a result, models may contain musculoskeletal systems that are not anatomically realistic. A musculoskeletal dataset measured from a single body will enable a complete and consistent model of the spine and is, therefore, a better approach for clinical practice [76].

Accuracy on modeling lines-of-action and architectural parameters of muscles in musculoskeletal models is of critical importance to yield reliable muscle and joint force estimations. Previous studies showed that models are very sensitive to the geometry of the spine [48, 87] and the changes in muscle attachment sites [51]. Furthermore, morphological (architectural) parameters such as fiber length, sarcomere length, optimal fiber length, tendon length, pennation angle, mass, and physiological cross-sectional area affect model predictions significantly [43, 88,-90]. These morphological parameters will facilitate more realistic simulation of muscle contraction dynamics in such models and, thus, will increase their credibility [35, 74].

In our previous study, we presented a complete and coherent musculoskeletal dataset for modeling the lumbar region of the spine [84]. Thus, the aim of this study is to obtain a complete and coherent musculoskeletal dataset for modeling the thoracic and cervical regions of the spine. We provide segmented bone surfaces, three-dimensional coordinates of muscle attachment sites, and the morphological muscle parameters measured from the same cadaver as in our previous study. When these two datasets are combined, a complete 
and consistent musculoskeletal model of the entire spine can be developed. The complete dataset is freely available through

https://www.utwente.nl/en/et/bw/research/projects/twentespinemodel.

\subsection{Materials and methods}

We obtained an embalmed human cadaver body (79 years-old male, height: $154 \mathrm{~cm}$, mass: $51 \mathrm{~kg}$ ) with institutional approval from Radboud university medical center. The cause of death was Alzheimer. We noticed slight scoliosis around the cadaver's neck (see Fig. 3.1 d). In the cadaver, we distinguished 43 bones: seven cervical, twelve thoracic, and four lumbar vertebrae, twelve ribs, skull, sternum, clavicle, scapula, hyoid, thyrohyoid, sacrum, and pelvis.

We measured muscles of the thoracic and cervical regions of the spine from the right side of the cadaver. The experimental method consisted of two parts and was described in detail in our previous study [84]. In the first part, we divided the muscles into a number of muscletendon elements to represent their function effectively. Subsequently, we dissected the muscle elements and measured the positions of their attachments at origin and insertion by using the NDI Hybrid Polaris Spectra tracking system. We additionally measured the positions of via points for elements with a curved lines-of-action. Muscle attachments were measured with respect to the corresponding reference frames of the bones, registered with the segmented CT images, and finally expressed with respect to the global reference frame (see Fig. 3.13 and b). The global reference frame was defined by the CT scanner, and in this frame $x$-, $y$-, and $z$-axes point point laterally (to the left side of the cadaver), posteriorly, and cranially, respectively (see Fig. 3.1 d). This reference frame (Cartesian coordinate system) used for reporting the coordinates is the same as the one used in our earlier study [84]. Finally, we labeled the resected elements and stored them in $2 \%$ formaldehyde solution until the measurement of the morphological parameters. In the second part, we measured the following morphological muscle parameters for every element: fiber length, tendon length, optimal fiber length, pennation angle, mass, and physiological cross-sectional area. An average sarcomere length was measured for every muscle by using the laser diffraction method [63], (see Fig. 3.1k).

The resection protocol was slightly different for longissimus cervicis, semispinalis thoracis, iliocostalis thoracis, and longissimus capitis muscles. For these muscles, firstly, attachments at the bones were measured, and the entire muscle was resected afterward. Subsequently, muscles were micro-dissected and were divided into elements.

Some muscles had intermediate tendons, connecting muscle bellies at either side of the muscle-tendon units, such as omohyoid (element 1), longissimus capitis (elements 1-3), 

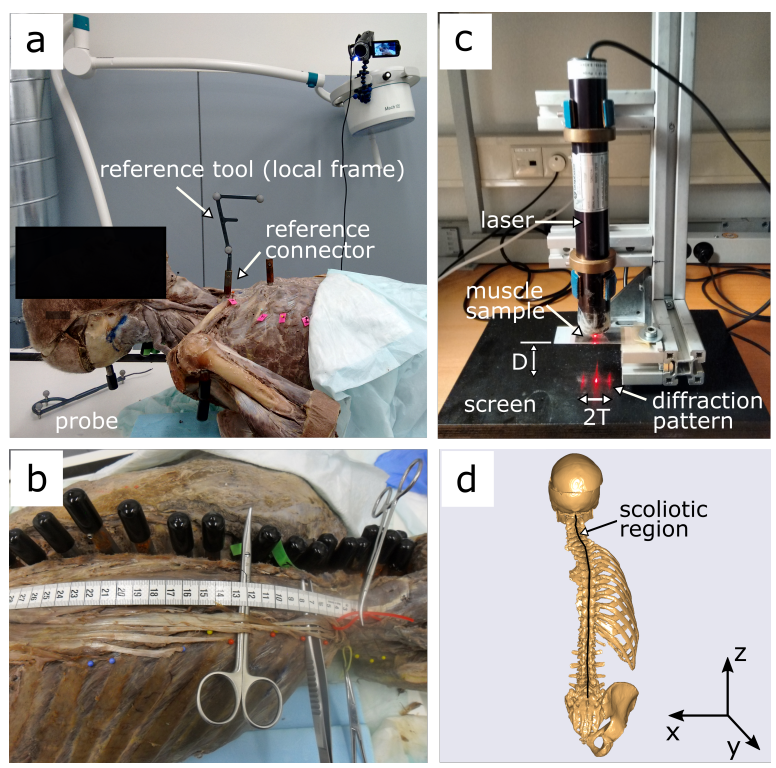

Figure 3.1: (a) An instance during position measurements showing the reference frames used for the bones and the probe. (b) Locating attachments of erector spinae muscle group. (c) Laser diffraction set-up used for sarcomere length measurements. (d) Visualization of the scoliosis in the cadaver. A curve which connects the spinous processes was drawn.

and semispinalis capitis (elements 1-3). For these elements, we measured fiber lengths and masses and calculated physiological cross-sectional areas (PCSA) of the two bellies separately. We reported the largest PCSA of the two bellies. The masses, average fiber lengths, and average optimal fiber lengths of the two bellies were summed, and the tendon length was calculated by subtracting total fiber length, multiplied by the cosine of the pennation angle, from the musculo-tendon length.

We discovered that some deeper muscles of the trunk such as the elements of the rotatores, levatores costarum, multifidus, and spinalis thoracis, differed in architecture as illustrated in Fig. 3.2. For example, the majority of the muscles reported in the present study had tendon-muscle-tendon architecture, (see Fig. 3.2 a). On the other hand, some deeper muscles had their tendons running from their origin to insertion. In these muscles, fibers either spanned (in parallel with the tendon) the full length of the tendon (rotatores and levatores costarum, see Fig. 3.2.) or partially spanned (most elements of the multifidus and spinalis thoracis, see Fig. 3.2 b). For these elements, we dissected tendon from the muscle and additionally calculated cross-sectional area (CSA, in $\mathrm{cm}^{2}$ ) of the tendon by using Eq. (1),

$$
C S A=\frac{m^{t}}{\rho^{t} \times \ell^{t}}
$$

where $\mathrm{m}^{t}$ is the mass, $\rho^{t}$ is the density, and $\ell^{t}$ is the length of the tendon. We calculated 

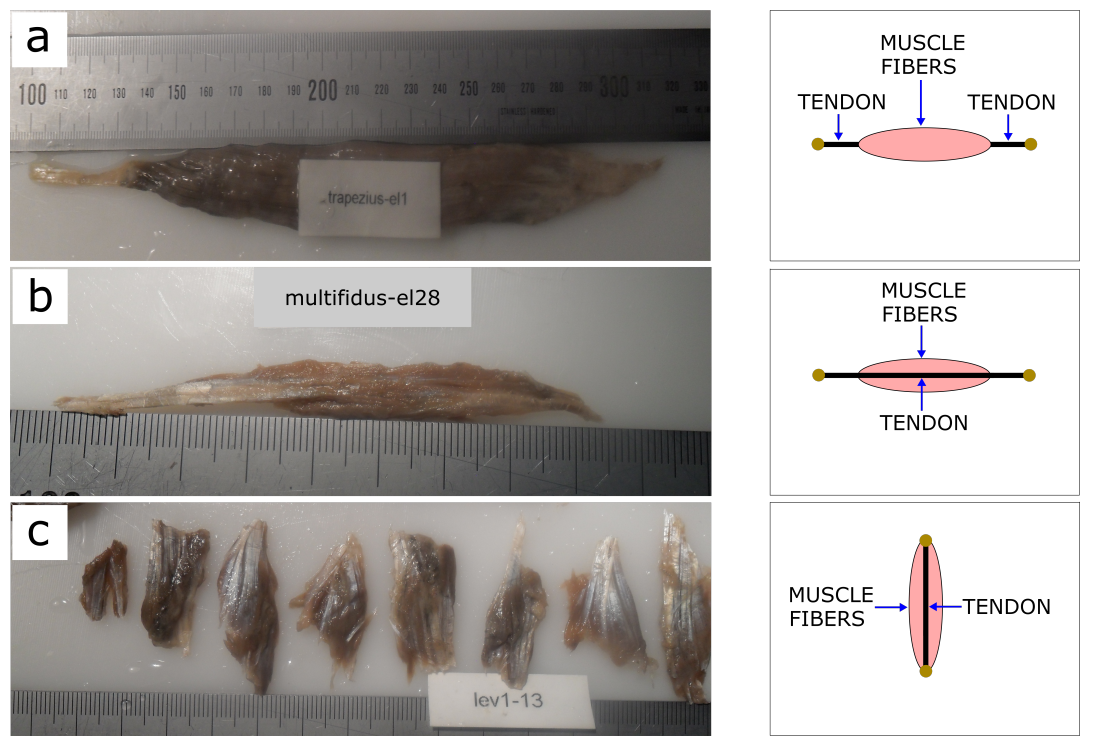

Figure 3.2: Illustration of the muscle architecture measured in this study. (a) Proximal and distal tendons are joined by the muscle fibers (tendon-muscle-tendon architecture). (b) Muscle fibers run in parallel with the tendon, but do not span the total length of the tendon. (c) Muscle fibers run in parallel with the tendon and fully span.

the average density of the tendon tissue $\left(1.2041 \mathrm{~g} / \mathrm{cm}^{3}\right)$ by dividing its mass by its volume measured using water dislocation method. Such muscle elements are indicated in Table 3.1 and Appendix B.3.

To reduce the study time, we performed measurements only between ribs 1 and 2, 5 and 6 , and 9 and 10 for intercostales muscles. Similarly, we measured levatores costarum (longi) only at the upper and lower rib levels.

Finally, we graphically fitted wrapping surfaces-from the geometry of the spine and the point clouds which were collected over the structures that muscles wrap-in the AnyBody Modeling System ${ }^{T M}$ version. 6.0.4 (AnyBody Technology A/S, Aalborg, Denmark). Depending on the shape of a surface, either a cylinder or an ellipsoid was fitted.

\subsection{Results}

The complete list of measured muscle elements is given in Table 3.1. In total, 225 muscle elements were measured for 39 muscles. For every element, we provided the coordinates of its attachments at origin and insertion and the morphological parameters: fiber length, sarcomere length, optimal fiber length, tendon length, pennation angle, mass, and physiological cross-sectional area (PCSA). Total muscle PCSAs ranged from $0.09 \mathrm{~cm}^{2}$ for sternothyroid 
muscle to $14.27 \mathrm{~cm}^{2}$ for trapezius muscle. Mean sarcomere lengths ranged from $2.10 \mu \mathrm{m}$ for the sternothyroid muscle to $3.91 \mu \mathrm{m}$ for semispinalis cervicis muscle. Mean optimal fiber lengths ranged from $0.6 \mathrm{~cm}$ for rectus capitis lateralis muscle to $14.7 \mathrm{~cm}$ for serratus anterior muscle. Mean tendon lengths ranged from $0.4 \mathrm{~cm}$ for the sternohyoid muscle to $16.4 \mathrm{~cm}$ for spinalis thoracis muscle. The coordinates of the via points, the mathematical definitions of the wrapping surfaces, and the architectural tendon parameters can be found in Appendix B.1 B.2, and B.3 respectively as digital appendices. All the bone segments with re-constructed muscle lines-of-action were visualized in the AnyBody Modeling System ${ }^{T M}$ ver. 6.0.4 (AnyBody Technology A/S, Aalborg, Denmark) and are depicted in Fig. 3.3
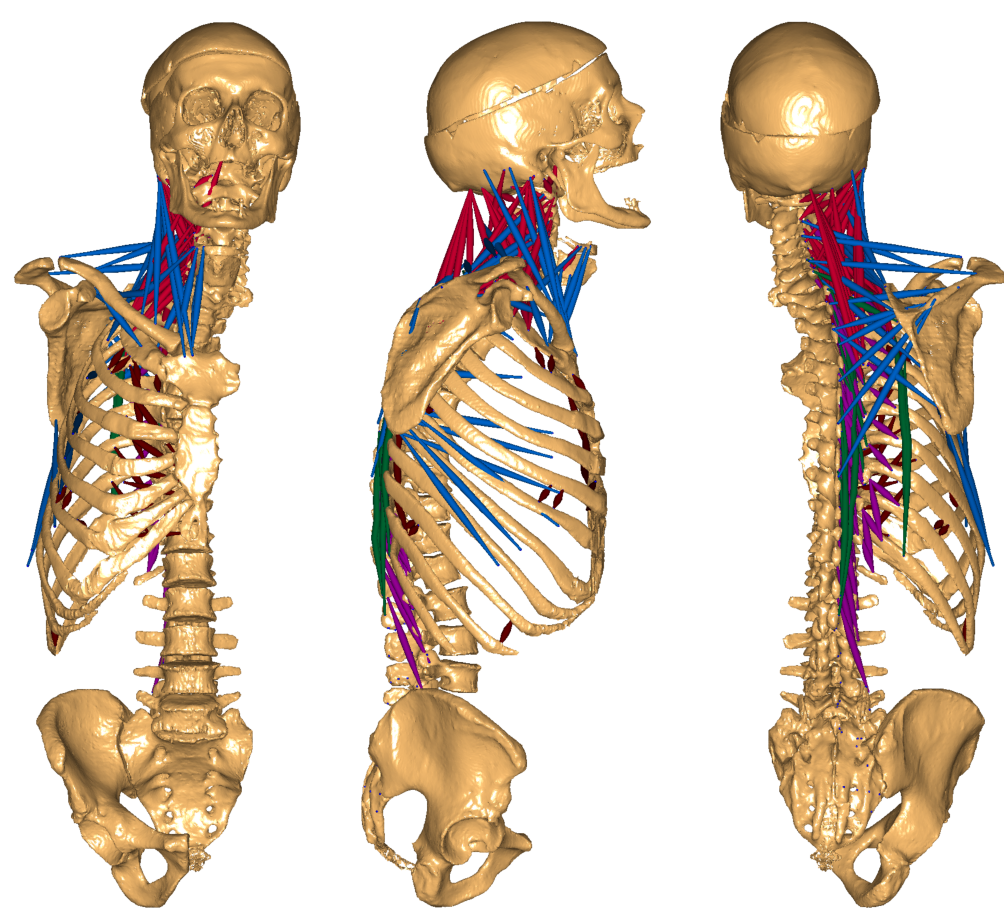

Figure 3.3: From left to right: anterior, lateral, and posterior views of the measured musculoskeletal system. Dissected muscles were highlighted in color: muscles of the thoracic spine (green), cervical spine (pale red), thoracic cage (dark red); deep muscles of the spine and ribcage (purple), and muscles connect spine to scapula, clavicle, and sternum (blue). 
Table 3.1: Per muscle element: element number (\#), fiber length $\left(\ell^{f}\right)$, sarcomere length $\left(\ell^{s}\right)$, optimal fiber length $\left(\ell_{o}^{f}\right)$, tendon length $\left(\ell^{t}\right)$, pennation angle $(\alpha)$, physiological cross-sectional area (PCSA), and the coordinates of the attachments at the origin and insertion with respect to the global reference frame defined by the CT scanner ${ }^{\mathrm{a}, \mathrm{d}, \mathrm{e}, \mathrm{f}}$.

\begin{tabular}{|c|c|c|c|c|c|c|c|c|c|c|c|c|c|c|c|c|c|c|}
\hline \multirow[t]{2}{*}{ Muscle } & \multirow[t]{2}{*}{$\#$} & \multirow{2}{*}{$\begin{array}{l}\ell f \\
(\mathrm{~mm})\end{array}$} & \multirow{2}{*}{$\begin{array}{l}\ell^{s} \\
(\mu \mathrm{m})\end{array}$} & \multirow{2}{*}{$\begin{array}{l}\ell_{o}^{f} \\
(\mathrm{~mm})\end{array}$} & \multirow{2}{*}{$\begin{array}{l}\ell^{t} \\
(\mathrm{~mm})\end{array}$} & \multirow{2}{*}{$\begin{array}{l}\alpha \\
\text { (deg) }\end{array}$} & \multirow{2}{*}{$\begin{array}{l}\text { Mass } \\
\text { (g) }\end{array}$} & \multirow{2}{*}{$\begin{array}{l}\text { PCSA } \\
\left(\mathrm{cm}^{2}\right)\end{array}$} & \multirow{2}{*}{$\begin{array}{l}\text { Origin } \\
\text { (bone) }\end{array}$} & \multirow[t]{2}{*}{ Form } & \multicolumn{3}{|c|}{ Position (m) } & \multirow{2}{*}{$\begin{array}{l}\text { Insertion } \\
\text { (bone) }\end{array}$} & \multirow[t]{2}{*}{ Form } & \multicolumn{3}{|c|}{ Position (m) } \\
\hline & & & & & & & & & & & $\mathrm{x}$ & $\mathrm{y}$ & $\mathrm{z}$ & & & $\mathrm{x}$ & $\mathrm{y}$ & $\mathrm{z}$ \\
\hline Iliocostalis cervicis ${ }^{h}$ & 1 & 57.7 & 3.39 & 45.9 & 106.3 & 0 & 0.65 & 0.13 & R5 & Point & -0.0603 & -0.0682 & 1.0304 & C5 & Point & 0.0024 & -0.1809 & 1.1259 \\
\hline Iliocostalis cervicish & 2 & 57.7 & 3.39 & 45.9 & 75.3 & 0 & 0.65 & 0.13 & $\mathrm{R} 4$ & Point & -0.0513 & -0.0876 & 1.0648 & C5 & Point & 0.0024 & -0.1809 & 1.1259 \\
\hline Iliocostalis cervicis ${ }^{h}$ & 3 & 57.7 & 3.39 & 45.9 & 54.3 & 0 & 0.65 & 0.13 & $\mathrm{R} 3$ & Point & -0.0466 & -0.1007 & 1.0741 & C5 & Point & 0.0024 & -0.1809 & 1.1259 \\
\hline Iliocostalis thoracis ${ }^{\mathrm{h}}$ & 1 & 147.5 & 2.81 & 141.6 & 59.0 & 0 & 2.85 & 0.19 & R11 & Point & -0.0659 & -0.0501 & 0.8636 & R5 & Point & -0.0665 & -0.0710 & 1.0394 \\
\hline Iliocostalis thoracis ${ }^{\mathrm{h}}$ & 2 & 147.5 & 2.81 & 141.6 & 88.5 & 0 & 2.85 & 0.19 & $\mathrm{R} 10$ & Point & -0.0668 & -0.0514 & 0.8931 & $\mathrm{R} 4$ & Point & -0.0544 & -0.0864 & 1.0631 \\
\hline Iliocostalis thoracis ${ }^{\mathrm{h}}$ & 3 & 147.5 & 2.81 & 141.6 & 80.0 & 0 & 2.85 & 0.19 & $\mathrm{R} 9$ & Point & -0.0684 & -0.0481 & 0.9254 & R3 & Point & -0.0483 & -0.1094 & 1.0810 \\
\hline Intercostales externi & 1 & 11.8 & 3.02 & 10.6 & 6.7 & 0 & 0.13 & 0.12 & $\mathrm{R} 2$ & Line & -0.0288 & -0.2262 & 1.0112 & $\mathrm{R} 1$ & Line & -0.0309 & -0.2148 & 1.0261 \\
\hline Intercostales externi & 2 & 13.3 & 3.02 & 11.9 & 14.7 & 0 & 2.50 & 1.99 & $\mathrm{R} 2$ & Line & -0.0738 & -0.1890 & 1.0319 & R1 & Line & -0.0602 & -0.1813 & 1.0502 \\
\hline Intercostales externi & 3 & 13.5 & 3.02 & 12.1 & 15.5 & 0 & 2.92 & 2.29 & $\mathrm{R} 2$ & Line & -0.0679 & -0.1377 & 1.0770 & $\mathrm{R} 1$ & Line & -0.0533 & -0.1524 & 1.0840 \\
\hline Intercostales externi & 4 & 20.3 & 3.02 & 18.1 & 15.8 & 0 & 1.46 & 0.76 & R6 & Line & -0.1206 & -0.1842 & 0.9117 & R5 & Line & -0.1121 & -0.1951 & 0.9263 \\
\hline Intercostales externi & 5 & 10.8 & 3.02 & 9.7 & 15.2 & 0 & 2.70 & 2.64 & R6 & Line & -0.0981 & -0.0796 & 0.9941 & R5 & Line & -0.0994 & -0.0854 & 1.0003 \\
\hline Intercostales externi & 6 & 15.7 & 3.02 & 14.0 & 10.3 & 0 & 3.01 & 2.03 & R10 & Line & -0.0972 & -0.0675 & 0.8819 & R9 & Line & -0.1060 & -0.0760 & 0.8907 \\
\hline Intercostales interni & 1 & 7.5 & 2.36 & 8.6 & 16.5 & 0 & 0.96 & 1.06 & $\mathrm{R} 2$ & Line & -0.0709 & -0.1949 & 1.0282 & $\mathrm{R} 1$ & Line & -0.0546 & -0.1956 & 1.0430 \\
\hline Intercostales interni & 2 & 13.7 & 2.36 & 15.7 & 9.3 & 0 & 1.83 & 1.11 & R6 & Line & -0.1149 & -0.1974 & 0.9060 & R5 & Line & -0.1073 & -0.2055 & 0.9221 \\
\hline Intercostales interni & 3 & 13.7 & 2.36 & 15.7 & 9.3 & 0 & 0.77 & 0.47 & R6 & Line & -0.0978 & -0.0816 & 0.9941 & R5 & Line & -0.1027 & -0.0895 & 0.9941 \\
\hline Intercostales interni & 4 & 20.6 & 2.36 & 23.6 & 3.4 & 0 & 4.31 & 1.73 & R10 & Line & -0.1218 & -0.1489 & 0.7829 & R9 & Line & -0.1221 & -0.1609 & 0.8070 \\
\hline Intercostales interni & 5 & 14.6 & 2.36 & 16.8 & 8.9 & 0 & 0.68 & 0.38 & $\mathrm{R} 10$ & Line & -0.0933 & -0.0638 & 0.8878 & R9 & Line & -0.1019 & -0.0748 & 0.8959 \\
\hline Levator scapulae & 1 & 127.3 & 3.04 & 113.1 & 31.7 & 0 & 4.62 & 0.39 & $\mathrm{C} 4$ & Point & -0.0003 & -0.1894 & 1.1401 & Scapula & Surface & -0.0533 & -0.1029 & 1.1003 \\
\hline Levator scapulae & 2 & 126.5 & 3.04 & 112.4 & 16.0 & 0 & 1.83 & 0.15 & C3 & Point & 0.0029 & -0.1931 & 1.1540 & Scapula & Surface & -0.0533 & -0.1029 & 1.1003 \\
\hline Levator scapulae & 3 & 118.8 & 3.04 & 105.5 & 20.3 & 0 & 5.11 & 0.46 & $\mathrm{C} 2$ & Point & 0.0038 & -0.1948 & 1.1641 & Scapula & Surface & -0.0533 & -0.1029 & 1.1003 \\
\hline Levator scapulae & 4 & 145.3 & 3.04 & 129.1 & 37.8 & 0 & 13.48 & 0.99 & $\mathrm{C} 1$ & Point & -0.0080 & -0.1883 & 1.1882 & Scapula & Surface & -0.0533 & -0.1029 & 1.1003 \\
\hline Levatores costarum ${ }^{\mathrm{c}}$ & 1 & 28.0 & 2.96 & 25.6 & 28.0 & 0 & 0.10 & 0.04 & $\mathrm{~T} 2$ & Point & -0.0251 & -0.1221 & 1.0961 & R3 & Point & -0.0436 & -0.1076 & 1.0827 \\
\hline Levatores costarum ${ }^{c, f}$ & 2 & 49.0 & 2.96 & 44.7 & 49.0 & 0 & 0.14 & 0.03 & $\mathrm{~T} 2$ & Point & -0.0251 & -0.1221 & 1.0961 & $\mathrm{R} 4$ & Point & -0.0523 & -0.0892 & 1.0648 \\
\hline Levatores costarum ${ }^{\mathrm{c}}$ & 3 & 29.0 & 2.96 & 26.5 & 29.0 & 0 & 0.12 & 0.04 & $\mathrm{~T} 3$ & Point & -0.0277 & -0.1061 & 1.0797 & $\mathrm{R} 4$ & Point & -0.0468 & -0.0876 & 1.0648 \\
\hline Levatores costarum ${ }^{\mathrm{c}}$ & 4 & 29.0 & 2.96 & 26.5 & 29.0 & 0 & 0.24 & 0.09 & $\mathrm{~T} 4$ & Point & -0.0308 & -0.0897 & 1.0597 & R5 & Point & -0.0523 & -0.0741 & 1.0447 \\
\hline Levatores costarum ${ }^{\mathrm{c}}$ & 5 & 26.0 & 2.96 & 23.7 & 26.0 & 0 & 0.06 & 0.02 & T5 & Point & -0.0342 & -0.0749 & 1.0368 & R6 & Point & -0.0534 & -0.0630 & 1.0220 \\
\hline Levatores costarum ${ }^{\mathrm{c}}$ & 6 & 33.0 & 2.96 & 30.1 & 33.0 & 0 & 0.12 & 0.04 & $\mathrm{~T} 6$ & Point & -0.0345 & -0.0619 & 1.0151 & $\mathrm{R} 7$ & Point & -0.0582 & -0.0539 & 0.9968 \\
\hline
\end{tabular}

Continued on next page 
Table 3.1: Continued from previous page

\begin{tabular}{|c|c|c|c|c|c|c|c|c|c|c|c|c|c|c|c|c|c|c|}
\hline \multirow[t]{2}{*}{ Muscle } & \multirow[t]{2}{*}{$\#$} & \multirow{2}{*}{$\begin{array}{l}\ell f \\
(\mathrm{~mm})\end{array}$} & \multirow{2}{*}{$\begin{array}{l}\ell^{s} \\
(\mu \mathrm{m})\end{array}$} & \multirow{2}{*}{$\begin{array}{l}\ell_{o}^{f} \\
(\mathrm{~mm})\end{array}$} & \multirow{2}{*}{$\begin{array}{l}\ell^{t} \\
(\mathrm{~mm})\end{array}$} & \multirow{2}{*}{$\begin{array}{l}\alpha \\
\text { (deg) }\end{array}$} & \multirow{2}{*}{$\begin{array}{l}\text { Mass } \\
\text { (g) }\end{array}$} & \multirow{2}{*}{$\begin{array}{l}\text { PCSA } \\
\left(\mathrm{cm}^{2}\right)\end{array}$} & \multirow{2}{*}{$\begin{array}{l}\text { Origin } \\
\text { (bone) }\end{array}$} & \multirow[t]{2}{*}{ Form } & \multicolumn{3}{|c|}{ Position (m) } & \multirow{2}{*}{$\begin{array}{l}\text { Insertion } \\
\text { (bone) }\end{array}$} & \multirow[t]{2}{*}{ Form } & \multicolumn{3}{|c|}{ Position (m) } \\
\hline & & & & & & & & & & & $\mathrm{x}$ & $\mathrm{y}$ & $\mathrm{z}$ & & & $\mathrm{x}$ & $\mathrm{y}$ & $\mathrm{z}$ \\
\hline Levatores costarum ${ }^{c}$ & 7 & 39.0 & 2.96 & 35.6 & 39.0 & 0 & 0.08 & 0.02 & $\mathrm{~T} 7$ & Point & -0.0340 & -0.0537 & 0.9880 & $\mathrm{R} 8$ & Point & -0.0603 & -0.0471 & 0.9689 \\
\hline Levatores costarum ${ }^{\mathrm{c}}$ & 8 & 35.0 & 2.96 & 32.0 & 35.0 & 0 & 0.12 & 0.04 & $\mathrm{~T} 8$ & Point & -0.0347 & -0.0487 & 0.9636 & R9 & Point & -0.0551 & -0.0440 & 0.9406 \\
\hline Levatores costarum $^{c}$ & 9 & 44.0 & 2.96 & 40.2 & 44.0 & 0 & 0.18 & 0.04 & T9 & Point & -0.0353 & -0.0466 & 0.9320 & $\mathrm{R} 10$ & Point & -0.0616 & -0.0461 & 0.9088 \\
\hline Levatores costarum ${ }^{\mathrm{c}, \mathrm{f}}$ & 10 & 59.0 & 2.96 & 53.9 & 59.0 & 0 & 0.85 & 0.15 & T9 & Point & -0.0353 & -0.0466 & 0.9320 & R11 & Point & -0.0462 & -0.0527 & 0.8772 \\
\hline Levatores costarum $^{c}$ & 11 & 42.0 & 2.96 & 38.3 & 42.0 & 0 & 0.46 & 0.11 & T10 & Point & -0.0290 & -0.0503 & 0.9056 & R11 & Point & -0.0462 & -0.0527 & 0.8772 \\
\hline Levatores $\operatorname{costarum}^{\mathrm{c}, \mathrm{f}}$ & 12 & 72.0 & 2.96 & 65.7 & 72.0 & 0 & 0.14 & 0.02 & T10 & Point & -0.0290 & -0.0503 & 0.9056 & R12 & Point & -0.0383 & -0.0685 & 0.8488 \\
\hline Levatores costarum $^{\mathrm{c}}$ & 13 & 40.0 & 2.96 & 36.5 & 40.0 & 0 & 0.30 & 0.08 & T11 & Point & -0.0288 & -0.0553 & 0.8777 & R12 & Point & -0.0383 & -0.0685 & 0.8488 \\
\hline Longissimus capitis & 1 & 115.0 & 2.81 & 110.4 & 120.0 & 0 & 0.85 & 0.09 & T5 & Point & -0.0295 & -0.0729 & 1.0354 & Skull & Line & -0.0244 & -0.1746 & 1.2005 \\
\hline Longissimus capitis & 2 & 115.0 & 2.81 & 110.4 & 102.0 & 0 & 0.85 & 0.09 & $\mathrm{~T} 4$ & Point & -0.0267 & -0.0883 & 1.0592 & Skull & Line & -0.0244 & -0.1746 & 1.2005 \\
\hline Longissimus capitis & 3 & 115.0 & 2.81 & 110.4 & 96.0 & 0 & 0.85 & 0.09 & T3 & Point & -0.0230 & -0.1039 & 1.0790 & Skull & Line & -0.0244 & -0.1746 & 1.2005 \\
\hline Longissimus capitis & 4 & 39.5 & 2.81 & 37.9 & 51.5 & 0 & 0.34 & 0.08 & $\mathrm{C} 7$ & Point & -0.0129 & -0.1646 & 1.1168 & Skull & Line & -0.0244 & -0.1746 & 1.2005 \\
\hline Longissimus capitis & 5 & 39.5 & 2.81 & 37.9 & 43.5 & 0 & 0.34 & 0.08 & $\mathrm{C} 5$ & Point & -0.0003 & -0.1818 & 1.1262 & Skull & Line & -0.0244 & -0.1746 & 1.2005 \\
\hline Longissimus capitis & 6 & 39.5 & 2.81 & 37.9 & 40.0 & 0 & 0.34 & 0.08 & $\mathrm{C} 4$ & Point & 0.0007 & -0.1914 & 1.1417 & Skull & Line & -0.0244 & -0.1746 & 1.2005 \\
\hline Longissimus capitis & 7 & 39.5 & 2.81 & 37.9 & 36.7 & 14 & 0.34 & 0.08 & $\mathrm{C} 3$ & Point & 0.0045 & -0.1929 & 1.1550 & Skull & Line & -0.0244 & -0.1746 & 1.2005 \\
\hline Longissimus cervicis & 1 & 45.5 & 3.70 & 33.2 & 0.5 & 0 & 0.68 & 0.19 & $\mathrm{~T} 1$ & Point & -0.0222 & -0.1453 & 1.1100 & C5 & Point & -0.0003 & -0.1818 & 1.1262 \\
\hline Longissimus cervicis & 2 & 45.5 & 3.70 & 33.2 & 16.0 & 0 & 0.68 & 0.19 & $\mathrm{~T} 2$ & Point & -0.0209 & -0.1211 & 1.0978 & $\mathrm{C} 4$ & Point & 0.0007 & -0.1914 & 1.1417 \\
\hline Longissimus cervicis & 3 & 45.5 & 3.70 & 33.2 & 46.5 & 0 & 0.68 & 0.19 & T3 & Point & -0.0150 & -0.1108 & 1.0779 & $\mathrm{C} 3$ & Point & 0.0045 & -0.1929 & 1.1550 \\
\hline Longissimus cervicis & 4 & 45.5 & 3.70 & 33.2 & 71.5 & 0 & 0.68 & 0.19 & T5 & Point & -0.0336 & -0.0752 & 1.0346 & $\mathrm{C} 7$ & Point & -0.0129 & -0.1646 & 1.1168 \\
\hline Longissimus cervicis & 5 & 36.8 & 3.70 & 26.8 & 118.3 & 0 & 0.82 & 0.29 & $\mathrm{~T} 6$ & Point & -0.0320 & -0.0618 & 1.0133 & $\mathrm{C} 6$ & Point & -0.0043 & -0.1755 & 1.1207 \\
\hline Longissimus cervicis & 6 & 42.3 & 3.70 & 30.9 & 93.7 & 0 & 0.95 & 0.29 & $\mathrm{~T} 4$ & Point & -0.0279 & -0.0896 & 1.0562 & $\mathrm{C} 5$ & Point & -0.0003 & -0.1818 & 1.1262 \\
\hline Longus capitis & 1 & 37.1 & 2.58 & 38.8 & 85.9 & 0 & 0.81 & 0.20 & $\mathrm{C} 6$ & Point & -0.0034 & -0.1785 & 1.1090 & Skull & Line & 0.0280 & -0.2000 & 1.2200 \\
\hline Longus capitis & 2 & 37.1 & 2.58 & 38.8 & 60.9 & 0 & 0.81 & 0.20 & $\mathrm{C} 5$ & Point & -0.0041 & -0.1864 & 1.1250 & Skull & Line & 0.0280 & -0.2000 & 1.2200 \\
\hline Longus capitis & 3 & 25.5 & 2.58 & 26.6 & 52.5 & 0 & 0.77 & 0.27 & $\mathrm{C} 4$ & Point & 0.0001 & -0.1902 & 1.1383 & Skull & Line & 0.0280 & -0.2000 & 1.2200 \\
\hline Longus capitis & 4 & 27.0 & 2.58 & 28.2 & 26.0 & 0 & 0.66 & 0.22 & $\mathrm{C} 3$ & Point & 0.0041 & -0.1934 & 1.1503 & Skull & Line & 0.0280 & -0.2000 & 1.2200 \\
\hline Multifidus & 16 & 61.0 & 3.42 & 48.2 & 25.0 & 0 & 1.07 & 0.21 & L4 & Point & -0.0341 & -0.0799 & 0.7411 & $\mathrm{~T} 12$ & Point & -0.0066 & -0.0485 & 0.8251 \\
\hline Multifidus & 17 & 69.8 & 3.42 & 55.1 & 3.8 & 0 & 1.90 & 0.33 & L3 & Point & -0.0244 & -0.0857 & 0.7673 & T11 & Point & -0.0062 & -0.0424 & 0.8538 \\
\hline Multifidus & 18 & 67.0 & 3.42 & 53.0 & 21.5 & 0 & 0.88 & 0.16 & $\mathrm{~L} 2$ & Point & -0.0251 & -0.0790 & 0.7874 & T10 & Point & -0.0076 & -0.0376 & 0.8773 \\
\hline Multifidus ${ }^{b}$ & 19 & 56.5 & 3.42 & 44.7 & 95.0 & 0 & 0.26 & 0.06 & L1 & Point & -0.0248 & -0.0706 & 0.8155 & T9 & Point & -0.0080 & -0.0372 & 0.9070 \\
\hline Multifidus ${ }^{b}$ & 20 & 45.5 & 3.42 & 36.0 & 113.5 & 0 & 1.89 & 0.50 & L1 & Point & -0.0248 & -0.0710 & 0.8152 & $\mathrm{~T} 8$ & Point & -0.0068 & -0.0395 & 0.9357 \\
\hline Multifidus ${ }^{b}$ & 21 & 82.0 & 3.42 & 64.8 & 139.0 & 0 & 0.20 & 0.03 & L1 & Point & -0.0248 & -0.0710 & 0.8152 & $\mathrm{~T} 7$ & Point & -0.0088 & -0.0455 & 0.9590 \\
\hline Multifidus $^{\mathrm{c}}$ & 22 & 41.0 & 3.42 & 32.4 & 41.0 & 0 & 0.14 & 0.04 & T12 & Point & -0.0181 & -0.0541 & 0.8560 & T9 & Point & -0.0083 & -0.0387 & 0.8971 \\
\hline Multifidus ${ }^{b}$ & 23 & 35.0 & 3.42 & 27.7 & 53.0 & 0 & 0.19 & 0.06 & T12 & Point & -0.0185 & -0.0554 & 0.8577 & T9 & Point & -0.0089 & -0.0418 & 0.9042 \\
\hline
\end{tabular}

Continued on next page 
Table 3.1: Continued from previous page

\begin{tabular}{|c|c|c|c|c|c|c|c|c|c|c|c|c|c|c|c|c|c|c|}
\hline \multirow[t]{2}{*}{ Muscle } & \multirow[t]{2}{*}{$\#$} & \multirow{2}{*}{$\begin{array}{l}\ell f \\
(\mathrm{~mm})\end{array}$} & \multirow{2}{*}{$\begin{array}{l}\ell^{s} \\
(\mu \mathrm{m})\end{array}$} & \multirow{2}{*}{$\begin{array}{l}\ell_{o}^{f} \\
(\mathrm{~mm})\end{array}$} & \multirow{2}{*}{$\begin{array}{l}\ell^{t} \\
(\mathrm{~mm})\end{array}$} & \multirow{2}{*}{$\begin{array}{l}\alpha \\
\text { (deg) }\end{array}$} & \multirow{2}{*}{$\begin{array}{l}\text { Mass } \\
\text { (g) }\end{array}$} & \multirow{2}{*}{$\begin{array}{l}\text { PCSA } \\
\left(\mathrm{cm}^{2}\right)\end{array}$} & \multirow{2}{*}{$\begin{array}{l}\text { Origin } \\
\text { (bone) }\end{array}$} & \multirow[t]{2}{*}{ Form } & \multicolumn{3}{|c|}{ Position (m) } & \multirow{2}{*}{$\begin{array}{l}\text { Insertion } \\
\text { (bone) }\end{array}$} & \multirow[t]{2}{*}{ Form } & \multicolumn{3}{|c|}{ Position (m) } \\
\hline & & & & & & & & & & & $\mathrm{x}$ & $\mathrm{y}$ & $\mathrm{z}$ & & & $\mathrm{x}$ & $\mathrm{y}$ & $\mathrm{z}$ \\
\hline Multifidus ${ }^{b}$ & 24 & 40.0 & 3.42 & 31.6 & 102.0 & 0 & 0.21 & 0.06 & $\mathrm{~T} 12$ & Point & -0.0189 & -0.0540 & 0.8561 & $\mathrm{~T} 7$ & Point & -0.0080 & -0.0386 & 0.9412 \\
\hline Multifidus $^{b}$ & 25 & 32.0 & 3.42 & 25.3 & 79.0 & 0 & 0.07 & 0.03 & T12 & Point & -0.0188 & -0.0564 & 0.8503 & $\mathrm{~T} 6$ & Point & -0.0100 & -0.0421 & 0.9702 \\
\hline Multifidus $^{b}$ & 26 & 38.0 & 3.42 & 30.0 & 47.0 & 0 & 0.03 & 0.01 & T11 & Point & -0.0215 & -0.0512 & 0.8836 & $\mathrm{~T} 8$ & Point & -0.0091 & -0.0387 & 0.9231 \\
\hline Multifidus $^{b}$ & 27 & 22.0 & 3.42 & 17.4 & 57.0 & 0 & 0.07 & 0.04 & T11 & Point & -0.0247 & -0.0516 & 0.8826 & $\mathrm{~T} 7$ & Point & -0.0077 & -0.0387 & 0.9405 \\
\hline Multifidus $^{b}$ & 28 & 37.0 & 3.42 & 29.2 & 84.5 & 0 & 0.44 & 0.14 & T11 & Point & -0.0247 & -0.0516 & 0.8826 & $\mathrm{~T} 6$ & Point & -0.0103 & -0.0418 & 0.9719 \\
\hline Multifidus $^{b}$ & 29 & 22.0 & 3.42 & 17.4 & 39.5 & 0 & 0.38 & 0.21 & T10 & Point & -0.0204 & -0.0523 & 0.9080 & $\mathrm{~T} 7$ & Point & -0.0091 & -0.0435 & 0.9529 \\
\hline Multifidus $^{b}$ & 30 & 43.0 & 3.42 & 34.0 & 61.5 & 0 & 0.11 & 0.03 & T10 & Point & -0.0214 & -0.0516 & 0.9142 & $\mathrm{~T} 6$ & Point & -0.0101 & -0.0457 & 0.9805 \\
\hline Multifidus $^{\mathrm{c}}$ & 31 & 35.5 & 3.42 & 28.1 & 35.5 & 0 & 0.04 & 0.01 & T10 & Point & -0.0243 & -0.0517 & 0.9157 & $\mathrm{~T} 7$ & Point & -0.0088 & -0.0474 & 0.9551 \\
\hline Multifidus $b$ & 32 & 19.0 & 3.42 & 15.0 & 47.0 & 0 & 0.01 & 0.01 & T9 & Point & -0.0299 & -0.0464 & 0.9274 & $\mathrm{~T} 6$ & Point & -0.0077 & -0.0426 & 0.9686 \\
\hline Multifidus $^{b}$ & 33 & 39.0 & 3.42 & 30.8 & 66.0 & 0 & 0.25 & 0.08 & T9 & Point & -0.0289 & -0.0445 & 0.9309 & $\mathrm{~T} 6$ & Point & -0.0080 & -0.0552 & 0.9997 \\
\hline Multifidus ${ }^{b}$ & 34 & 16.0 & 3.42 & 12.6 & 65.5 & 0 & 0.01 & 0.01 & T9 & Point & -0.0289 & -0.0445 & 0.9309 & T5 & Point & -0.0077 & -0.0670 & 1.0270 \\
\hline Multifidus $^{b}$ & 35 & 36.0 & 3.42 & 28.5 & 93.0 & 0 & 0.12 & 0.04 & T9 & Point & -0.0281 & -0.0457 & 0.9358 & $\mathrm{~T} 4$ & Point & -0.0122 & -0.0632 & 1.0343 \\
\hline Multifidus $b$ & 36 & 29.0 & 3.42 & 22.9 & 70.0 & 0 & 0.24 & 0.10 & T9 & Point & -0.0253 & -0.0489 & 0.9399 & T5 & Point & -0.0093 & -0.0511 & 1.0013 \\
\hline Multifidus ${ }^{b}$ & 37 & 21.0 & 3.42 & 16.6 & 37.0 & 0 & 0.05 & 0.03 & T9 & Point & -0.0256 & -0.0516 & 0.9417 & $\mathrm{~T} 6$ & Point & -0.0105 & -0.0494 & 0.9792 \\
\hline Multifidus $^{b}$ & 38 & 29.0 & 3.42 & 22.9 & 44.0 & 0 & 0.24 & 0.10 & T8 & Point & -0.0249 & -0.0526 & 0.9651 & T5 & Point & -0.0093 & -0.0511 & 1.0013 \\
\hline Multifidus $b$ & 39 & 25.0 & 3.42 & 19.8 & 44.0 & 0 & 0.11 & 0.05 & T8 & Point & -0.0249 & -0.0528 & 0.9662 & T5 & Point & -0.0103 & -0.0553 & 1.0109 \\
\hline Multifidus $^{b}$ & 40 & 74.0 & 3.42 & 58.5 & 105.0 & 0 & 0.40 & 0.06 & $\mathrm{~T} 8$ & Point & -0.0306 & -0.0496 & 0.9595 & $\mathrm{~T} 3$ & Point & -0.0066 & -0.0787 & 1.0599 \\
\hline Multifidus $^{b}$ & 41 & 34.0 & 3.42 & 26.9 & 67.0 & 0 & 0.38 & 0.13 & $\mathrm{~T} 8$ & Point & -0.0282 & -0.0545 & 0.9689 & $\mathrm{~T} 4$ & Point & -0.0123 & -0.0638 & 1.0357 \\
\hline Multifidus $b$ & 42 & 27.0 & 3.42 & 21.3 & 44.0 & 0 & 0.08 & 0.04 & $\mathrm{~T} 8$ & Point & -0.0263 & -0.0561 & 0.9688 & T5 & Point & -0.0101 & -0.0599 & 1.0088 \\
\hline Multifidus $^{b}$ & 43 & 53.0 & 3.42 & 41.9 & 85.0 & 0 & 0.23 & 0.05 & $\mathrm{~T} 7$ & Point & -0.0266 & -0.0562 & 0.9861 & $\mathrm{~T} 3$ & Point & -0.0061 & -0.0817 & 1.0608 \\
\hline Multifidus $^{b}$ & 44 & 45.0 & 3.42 & 35.6 & 100.5 & 0 & 0.48 & 0.13 & $\mathrm{~T} 7$ & Point & -0.0301 & -0.0529 & 0.9880 & $\mathrm{~T} 2$ & Point & -0.0024 & -0.0935 & 1.0828 \\
\hline Multifidus $b$ & 45 & 35.0 & 3.42 & 27.7 & 48.5 & 0 & 0.11 & 0.04 & $\mathrm{~T} 7$ & Point & -0.0240 & -0.0651 & 0.9931 & $\mathrm{~T} 4$ & Point & -0.0083 & -0.0749 & 1.0375 \\
\hline Multifidus $^{b}$ & 46 & 43.0 & 3.42 & 34.0 & 76.5 & 0 & 0.20 & 0.06 & $\mathrm{~T} 7$ & Point & -0.0296 & -0.0616 & 0.9938 & $\mathrm{~T} 3$ & Point & -0.0054 & -0.0826 & 1.0601 \\
\hline Multifidus $^{b}$ & 47 & 43.0 & 3.42 & 34.0 & 79.0 & 0 & 0.13 & 0.03 & T6 & Point & -0.0296 & -0.0635 & 1.0182 & $\mathrm{~T} 2$ & Point & -0.0024 & -0.0935 & 1.0828 \\
\hline Multifidus $^{b}$ & 48 & 43.0 & 3.42 & 34.0 & 101.0 & 0 & 0.13 & 0.03 & T6 & Point & -0.0296 & -0.0635 & 1.0182 & $\mathrm{~T} 1$ & Point & 0.0002 & -0.1071 & 1.0984 \\
\hline Multifidus $b$ & 49 & 35.0 & 3.42 & 27.7 & 76.5 & 0 & 0.18 & 0.06 & $\mathrm{~T} 6$ & Point & -0.0279 & -0.0690 & 1.0212 & $\mathrm{~T} 2$ & Point & -0.0011 & -0.0981 & 1.0818 \\
\hline Multifidus $b$ & 50 & 22.0 & 3.42 & 17.4 & 49.0 & 0 & 0.09 & 0.05 & $\mathrm{~T} 6$ & Point & -0.0280 & -0.0701 & 1.0210 & $\mathrm{~T} 3$ & Point & -0.0041 & -0.0912 & 1.0588 \\
\hline Multifidus $^{b}$ & 51 & 34.5 & 3.42 & 27.3 & 45.0 & 0 & 0.26 & 0.09 & T5 & Point & -0.0330 & -0.0775 & 1.0419 & $\mathrm{~T} 2$ & Point & -0.0005 & -0.1070 & 1.0799 \\
\hline Multifidus $^{b}$ & 52 & 34.5 & 3.42 & 27.3 & 61.0 & 0 & 0.26 & 0.09 & T5 & Point & -0.0330 & -0.0775 & 1.0419 & $\mathrm{~T} 1$ & Point & 0.0018 & -0.1128 & 1.0971 \\
\hline Multifidus ${ }^{c}$ & 53 & 37.5 & 3.42 & 29.6 & 37.5 & 0 & 0.10 & 0.03 & $\mathrm{~T} 4$ & Point & -0.0211 & -0.0980 & 1.0647 & $\mathrm{~T} 1$ & Point & 0.0046 & -0.1158 & 1.0944 \\
\hline Multifidus $^{b}$ & 54 & 51.0 & 3.42 & 40.3 & 66.0 & 0 & 0.81 & 0.19 & $\mathrm{~T} 4$ & Point & -0.0229 & -0.0962 & 1.0662 & C7 & Point & 0.0132 & -0.1304 & 1.1113 \\
\hline Multifidus $^{b}$ & 55 & 22.0 & 3.42 & 17.4 & 43.0 & 0 & 0.02 & 0.01 & T3 & Point & -0.0200 & -0.1145 & 1.0829 & $\mathrm{C} 7$ & Point & 0.0130 & -0.1295 & 1.1119 \\
\hline Multifidus $^{c}$ & 56 & 47.0 & 3.42 & 37.1 & 47.0 & 0 & 0.04 & 0.01 & T3 & Point & -0.0201 & -0.1156 & 1.0812 & $\mathrm{C} 7$ & Point & 0.0108 & -0.1364 & 1.1100 \\
\hline Multifidus ${ }^{b}$ & 57 & 20.0 & 3.42 & 15.8 & 39.5 & 0 & 0.19 & 0.11 & $\mathrm{~T} 2$ & Point & -0.0193 & -0.1322 & 1.0998 & $\mathrm{C} 6$ & Point & 0.0181 & -0.1433 & 1.1252 \\
\hline Multifidus $^{\mathrm{c}}$ & 58 & 34.0 & 3.42 & 26.9 & 34.0 & 0 & 0.61 & 0.21 & $\mathrm{~T} 1$ & Point & -0.0133 & -0.1481 & 1.1120 & $\mathrm{C} 6$ & Point & 0.0179 & -0.1461 & 1.1248 \\
\hline Multifidus $^{\mathrm{c}}$ & 59 & 48.0 & 3.42 & 37.9 & 48.0 & 0 & 0.58 & 0.14 & $\mathrm{~T} 1$ & Point & -0.0133 & -0.1481 & 1.1120 & $\mathrm{C} 4$ & Point & 0.0164 & -0.1528 & 1.1379 \\
\hline
\end{tabular}

Continued on next page 


\begin{tabular}{|c|c|c|c|c|c|c|c|c|c|c|c|c|c|c|c|c|c|c|}
\hline \multirow[t]{2}{*}{ Muscle } & \multirow[t]{2}{*}{$\#$} & \multirow{2}{*}{$\begin{array}{l}\ell f \\
(\mathrm{~mm})\end{array}$} & \multirow{2}{*}{$\begin{array}{l}\ell^{s} \\
(\mu \mathrm{m})\end{array}$} & \multirow{2}{*}{$\begin{array}{l}\ell_{o}^{f} \\
(\mathrm{~mm})\end{array}$} & \multirow{2}{*}{$\begin{array}{l}\ell^{t} \\
(\mathrm{~mm})\end{array}$} & \multirow{2}{*}{$\begin{array}{l}\alpha \\
(\operatorname{deg})\end{array}$} & \multirow{2}{*}{$\begin{array}{l}\text { Mass } \\
\text { (g) }\end{array}$} & \multirow{2}{*}{$\begin{array}{l}\text { PCSA } \\
\left(\mathrm{cm}^{2}\right)\end{array}$} & \multirow{2}{*}{$\begin{array}{l}\text { Origin } \\
\text { (bone) }\end{array}$} & \multirow[t]{2}{*}{ Form } & \multicolumn{3}{|c|}{ Position (m) } & \multirow{2}{*}{$\begin{array}{l}\text { Insertion } \\
\text { (bone) }\end{array}$} & \multirow[t]{2}{*}{ Form } & \multicolumn{3}{|c|}{ Position (m) } \\
\hline & & & & & & & & & & & $\mathrm{x}$ & $\mathrm{y}$ & $\mathrm{z}$ & & & $\mathrm{x}$ & $\mathrm{y}$ & $\mathrm{z}$ \\
\hline Multifidus ${ }^{b}$ & 60 & 31.0 & 3.42 & 24.5 & 44.5 & 0 & 0.97 & 0.37 & $\mathrm{~T} 1$ & Point & -0.0133 & -0.1481 & 1.1120 & $\mathrm{C} 4$ & Point & 0.0164 & -0.1528 & 1.1379 \\
\hline Multifidus ${ }^{b}$ & 61 & 23.0 & 3.42 & 18.2 & 33.0 & 0 & 0.65 & 0.34 & $\mathrm{C} 7$ & Point & -0.0018 & -0.1617 & 1.1191 & C5 & Point & 0.0178 & -0.1528 & 1.1357 \\
\hline Multifidus $^{\mathrm{c}}$ & 62 & 39.0 & 3.42 & 30.8 & 39.0 & 0 & 0.39 & 0.12 & $\mathrm{C} 7$ & Point & -0.0099 & -0.1659 & 1.1189 & $\mathrm{C} 4$ & Point & 0.0196 & -0.1551 & 1.1401 \\
\hline Multifidus ${ }^{\mathrm{c}}$ & 63 & 36.5 & 3.42 & 28.8 & 36.5 & 0 & 0.35 & 0.11 & $\mathrm{C} 5$ & Point & -0.0022 & -0.1679 & 1.1293 & $\mathrm{C} 3$ & Point & 0.0233 & -0.1618 & 1.1546 \\
\hline Multifidus ${ }^{\mathrm{c}}$ & 64 & 39.0 & 3.42 & 30.8 & 39.0 & 0 & 0.28 & 0.09 & $\mathrm{C} 4$ & Point & 0.0000 & -0.1771 & 1.1401 & $\mathrm{C} 2$ & Point & 0.0207 & -0.1620 & 1.1651 \\
\hline Obliquus capitis inferior & 1 & 35.3 & 2.69 & 35.3 & 12.8 & 0 & 5.40 & 1.45 & $\mathrm{C} 2$ & Surface & 0.0226 & -0.1641 & 1.1702 & $\mathrm{C} 1$ & Surface & -0.0034 & -0.1893 & 1.1850 \\
\hline Obliquus capitis superior & 1 & 21.3 & 3.16 & 18.2 & 33.8 & 0 & 0.63 & 0.33 & $\mathrm{C} 1$ & Surface & -0.0074 & -0.1900 & 1.1858 & Skull & Surface & -0.0097 & -0.1594 & 1.1951 \\
\hline Omohyoid $^{\text {h }}$ & 1 & 117.8 & 2.45 & 129.6 & 51.2 & 0 & 3.26 & 0.24 & Scapula & Line & -0.0829 & -0.1200 & 1.0966 & Hyoid & Line & 0.0034 & -0.2220 & 1.1412 \\
\hline Omohyoid & 2 & 50.5 & 2.45 & 55.6 & 21.0 & 0 & 0.38 & 0.06 & Scapula & Point & -0.0896 & -0.1356 & 1.1008 & Clavicle & Point & -0.0476 & -0.1934 & 1.0781 \\
\hline Rectus capitis anterior & 1 & 9.5 & 2.63 & 9.7 & 13.5 & 0 & 0.21 & 0.20 & $\mathrm{C} 1$ & Line & 0.0038 & -0.1941 & 1.1957 & Skull & Line & 0.0191 & -0.1944 & 1.2059 \\
\hline Rectus capitis lateralis $\mathrm{g}$ & 1 & 6.3 & 2.63 & 6.4 & 11.8 & 0 & 0.07 & 0.10 & $\mathrm{C} 1$ & Line & -0.0063 & -0.1950 & 1.1898 & Skull & Line & -0.0218 & -0.1887 & 1.2020 \\
\hline Rectus capitis posterior major & 1 & 29.0 & 2.83 & 27.7 & 10.0 & 0 & 3.04 & 1.04 & $\mathrm{C} 2$ & Point & 0.0272 & -0.1543 & 1.1661 & Skull & Surface & 0.0036 & -0.1479 & 1.1900 \\
\hline Rectus capitis posterior minor & 1 & 14.5 & 2.99 & 13.1 & 15.5 & 0 & 0.42 & 0.30 & $\mathrm{C} 1$ & Point & 0.0272 & -0.1642 & 1.1821 & Skull & Line & 0.0093 & -0.1479 & 1.1891 \\
\hline Rhomboideus major & 1 & 122.3 & 3.19 & 103.5 & 63.7 & 0 & 10.28 & 0.94 & T3 & Line & -0.0025 & -0.0779 & 1.0673 & Scapula & Line & -0.0958 & -0.0481 & 0.9980 \\
\hline Rhomboideus major & 2 & 115.2 & 3.19 & 97.5 & 60.8 & 0 & 23.16 & 2.25 & $\mathrm{~T} 1$ & Line & 0.0073 & -0.1132 & 1.1111 & Scapula & Line & -0.0807 & -0.0496 & 1.0340 \\
\hline Rhomboideus minor & 1 & 105.5 & 3.19 & 89.3 & 64.5 & 0 & 31.68 & 3.35 & $\mathrm{C} 6$ & Line & 0.0165 & -0.1214 & 1.1288 & Scapula & Line & -0.0571 & -0.0691 & 1.0837 \\
\hline Rotatores ${ }^{\mathrm{c}}$ & 1 & 30.0 & 3.03 & 26.7 & 30.0 & 0 & 0.14 & 0.05 & $\mathrm{~T} 12$ & Point & -0.0181 & -0.0541 & 0.8560 & $\mathrm{~T} 10$ & Point & -0.0076 & -0.0486 & 0.8842 \\
\hline Rotatores $^{\mathrm{c}}$ & 2 & 30.0 & 3.03 & 26.7 & 30.0 & 0 & 0.19 & 0.07 & $\mathrm{~T} 12$ & Point & -0.0185 & -0.0554 & 0.8577 & T10 & Point & -0.0111 & -0.0543 & 0.8877 \\
\hline Rotatores $\mathrm{s}^{\mathrm{c}}$ & 3 & 26.0 & 3.03 & 23.2 & 26.0 & 0 & 0.03 & 0.01 & T11 & Point & -0.0215 & -0.0512 & 0.8836 & T9 & Point & -0.0088 & -0.0446 & 0.9060 \\
\hline Rotatores $^{\mathrm{c}}$ & 4 & 23.0 & 3.03 & 20.5 & 23.0 & 0 & 0.10 & 0.05 & T11 & Point & -0.0193 & -0.0543 & 0.8838 & T9 & Point & -0.0071 & -0.0529 & 0.9093 \\
\hline Rotatores $\mathrm{c}^{\mathrm{c}}$ & 5 & 18.0 & 3.03 & 16.0 & 18.0 & 0 & 0.01 & 0.01 & T10 & Point & -0.0286 & -0.0532 & 0.9145 & T9 & Point & -0.0081 & -0.0507 & 0.9221 \\
\hline Rotatores ${ }^{c}$ & 6 & 20.0 & 3.03 & 17.8 & 20.0 & 0 & 0.02 & 0.01 & T10 & Point & -0.0199 & -0.0541 & 0.9133 & $\mathrm{~T} 8$ & Point & -0.0085 & -0.0492 & 0.9339 \\
\hline Rotatores $\mathrm{c}^{\mathrm{C}}$ & 7 & 26.0 & 3.03 & 23.2 & 26.0 & 0 & 0.19 & 0.08 & T9 & Point & -0.0231 & -0.0506 & 0.9366 & $\mathrm{~T} 7$ & Point & -0.0091 & -0.0521 & 0.9601 \\
\hline Rotatores $\mathrm{s}^{\mathrm{c}}$ & 8 & 13.0 & 3.03 & 11.6 & 13.0 & 0 & 0.02 & 0.02 & T9 & Point & -0.0201 & -0.0533 & 0.9351 & $\mathrm{~T} 8$ & Point & -0.0105 & -0.0522 & 0.9384 \\
\hline Rotatores $\mathrm{c}^{\mathrm{C}}$ & 9 & 15.0 & 3.03 & 13.4 & 15.0 & 0 & 0.19 & 0.13 & T9 & Point & -0.0201 & -0.0533 & 0.9351 & $\mathrm{~T} 8$ & Point & -0.0140 & -0.0552 & 0.9489 \\
\hline Rotatores $\mathrm{s}^{\mathrm{c}}$ & 10 & 8.0 & 3.03 & 7.1 & 8.0 & 0 & 0.03 & 0.03 & $\mathrm{~T} 8$ & Point & -0.0206 & -0.0603 & 0.9670 & $\mathrm{~T} 7$ & Point & -0.0127 & -0.0561 & 0.9668 \\
\hline Rotatores $^{\mathrm{c}}$ & 11 & 24.0 & 3.03 & 21.4 & 24.0 & 0 & 0.03 & 0.01 & T8 & Point & -0.0206 & -0.0603 & 0.9670 & $\mathrm{~T} 6$ & Point & -0.0102 & -0.0583 & 0.9879 \\
\hline
\end{tabular}

Continued on next page 
Table 3.1: Continued from previous page

\begin{tabular}{|c|c|c|c|c|c|c|c|c|c|c|c|c|c|c|c|c|c|c|}
\hline \multirow[t]{2}{*}{ Muscle } & \multirow[t]{2}{*}{$\#$} & \multirow{2}{*}{$\begin{array}{l}\ell f \\
(\mathrm{~mm})\end{array}$} & \multirow{2}{*}{$\begin{array}{l}\ell^{s} \\
(\mu \mathrm{m})\end{array}$} & \multirow{2}{*}{$\begin{array}{l}\ell_{o}^{f} \\
(\mathrm{~mm})\end{array}$} & \multirow{2}{*}{$\begin{array}{l}\ell^{t} \\
(\mathrm{~mm})\end{array}$} & \multirow{2}{*}{$\begin{array}{l}\alpha \\
(\operatorname{deg})\end{array}$} & \multirow{2}{*}{$\begin{array}{l}\text { Mass } \\
\text { (g) }\end{array}$} & \multirow{2}{*}{$\begin{array}{l}\text { PCSA } \\
\left(\mathrm{cm}^{2}\right)\end{array}$} & \multirow{2}{*}{$\begin{array}{l}\text { Origin } \\
\text { (bone) }\end{array}$} & \multirow[t]{2}{*}{ Form } & \multicolumn{3}{|c|}{ Position (m) } & \multirow{2}{*}{$\begin{array}{l}\text { Insertion } \\
\text { (bone) }\end{array}$} & \multirow[t]{2}{*}{ Form } & \multicolumn{3}{|c|}{ Position (m) } \\
\hline & & & & & & & & & & & $\mathrm{x}$ & $\mathrm{y}$ & $\mathrm{z}$ & & & $\mathrm{x}$ & $\mathrm{y}$ & $\mathrm{z}$ \\
\hline Rotatores $^{\mathrm{c}}$ & 12 & 8.0 & 3.03 & 7.1 & 8.0 & 0 & 0.06 & 0.07 & $\mathrm{~T} 7$ & Point & -0.0205 & -0.0617 & 0.9888 & T6 & Point & -0.0142 & -0.0632 & 0.9940 \\
\hline Rotatores $\mathrm{c}$ & 13 & 26.5 & 3.03 & 23.6 & 26.5 & 0 & 0.06 & 0.02 & $\mathrm{~T} 7$ & Point & -0.0255 & -0.0660 & 0.9931 & T5 & Point & -0.0115 & -0.0698 & 1.0166 \\
\hline Rotatores ${ }^{\mathrm{c}}$ & 14 & 9.0 & 3.03 & 8.0 & 9.0 & 0 & 0.03 & 0.04 & $\mathrm{~T} 6$ & Point & -0.0242 & -0.0711 & 1.0194 & T5 & Point & -0.0143 & -0.0725 & 1.0190 \\
\hline Rotatores ${ }^{c}$ & 15 & 38.0 & 3.03 & 33.9 & 38.0 & 0 & 0.03 & 0.01 & T6 & Point & -0.0199 & -0.0719 & 1.0163 & $\mathrm{~T} 4$ & Point & -0.0080 & -0.0826 & 1.0420 \\
\hline Rotatores $^{\mathrm{c}}$ & 16 & 9.0 & 3.03 & 8.0 & 9.0 & 0 & 0.03 & 0.04 & T5 & Point & -0.0189 & -0.0846 & 1.0403 & $\mathrm{~T} 4$ & Point & -0.0103 & -0.0849 & 1.0430 \\
\hline Rotatores $^{\mathrm{c}}$ & 17 & 27.5 & 3.03 & 24.5 & 27.5 & 0 & 0.03 & 0.01 & T5 & Point & -0.0244 & -0.0845 & 1.0420 & $\mathrm{~T} 3$ & Point & -0.0062 & -0.0986 & 1.0600 \\
\hline Rotatores $^{\mathrm{c}}$ & 18 & 7.0 & 3.03 & 6.2 & 7.0 & 0 & 0.05 & 0.07 & $\mathrm{~T} 4$ & Point & -0.0154 & -0.0984 & 1.0601 & $\mathrm{~T} 3$ & Point & -0.0082 & -0.1006 & 1.0608 \\
\hline Rotatores $\mathrm{c}$ & 19 & 26.5 & 3.03 & 23.6 & 26.5 & 0 & 0.05 & 0.02 & $\mathrm{~T} 4$ & Point & -0.0189 & -0.0991 & 1.0629 & $\mathrm{~T} 2$ & Point & -0.0004 & -0.1098 & 1.0780 \\
\hline Rotatores $^{\mathrm{c}}$ & 20 & 31.5 & 3.03 & 28.1 & 31.5 & 0 & 0.04 & 0.01 & $\mathrm{~T} 3$ & Point & -0.0218 & -0.1071 & 1.0832 & $\mathrm{~T} 1$ & Point & 0.0034 & -0.1218 & 1.0950 \\
\hline Rotatores $\mathrm{c}$ & 21 & 27.0 & 3.03 & 24.1 & 27.0 & 0 & 0.23 & 0.09 & $\mathrm{~T} 3$ & Point & -0.0131 & -0.1118 & 1.0760 & $\mathrm{~T} 1$ & Point & 0.0034 & -0.1218 & 1.0950 \\
\hline Rotatores $\mathrm{c}$ & 22 & 19.0 & 3.03 & 16.9 & 19.0 & 0 & 0.07 & 0.04 & $\mathrm{~T} 3$ & Point & -0.0163 & -0.1145 & 1.0801 & $\mathrm{~T} 1$ & Point & 0.0026 & -0.1257 & 1.0943 \\
\hline Rotatores ${ }^{\mathrm{c}}$ & 23 & 30.5 & 3.03 & 27.2 & 30.5 & 0 & 0.24 & 0.08 & $\mathrm{~T} 2$ & Point & -0.0161 & -0.1304 & 1.0979 & $\mathrm{C} 7$ & Point & 0.0081 & -0.1422 & 1.1106 \\
\hline Rotatores $^{\mathrm{c}}$ & 24 & 30.5 & 3.03 & 27.2 & 30.5 & 0 & 0.24 & 0.08 & $\mathrm{~T} 2$ & Point & -0.0142 & -0.1276 & 1.0951 & $\mathrm{C} 7$ & Point & 0.0091 & -0.1402 & 1.1083 \\
\hline Rotatores $^{\mathrm{c}}$ & 25 & 21.0 & 3.03 & 18.7 & 21.0 & 0 & 0.13 & 0.07 & $\mathrm{~T} 2$ & Point & -0.0142 & -0.1276 & 1.0951 & $\mathrm{~T} 1$ & Point & -0.0016 & -0.1319 & 1.0961 \\
\hline Rotatores $^{\mathrm{c}}$ & 26 & 27.5 & 3.03 & 24.5 & 27.5 & 0 & 0.15 & 0.06 & T1 & Point & -0.0115 & -0.1442 & 1.1098 & $\mathrm{C} 6$ & Point & 0.0180 & -0.1423 & 1.1251 \\
\hline Scalenus anterior & 1 & 25.7 & 2.37 & 29.2 & 34.3 & 0 & 0.89 & 0.29 & $\mathrm{C} 7$ & Point & -0.0120 & -0.1671 & 1.1044 & $\mathrm{R} 1$ & Surface & -0.0522 & -0.1747 & 1.0660 \\
\hline Scalenus anterior & 2 & 25.7 & 2.37 & 29.2 & 44.3 & 0 & 0.89 & 0.29 & C6 & Point & -0.0028 & -0.1784 & 1.1099 & R1 & Surface & -0.0522 & -0.1747 & 1.0660 \\
\hline Scalenus anterior & 3 & 25.7 & 2.37 & 29.2 & 41.3 & 0 & 0.89 & 0.29 & C5 & Point & -0.0022 & -0.1829 & 1.1234 & $\mathrm{R} 1$ & Surface & -0.0522 & -0.1747 & 1.0660 \\
\hline Scalenus medius & 1 & 22.3 & 2.65 & 22.7 & 44.8 & 0 & 2.38 & 0.99 & $\mathrm{C} 6$ & Point & -0.0068 & -0.1759 & 1.1191 & $\mathrm{R} 1$ & Surface & -0.0561 & -0.1608 & 1.0788 \\
\hline Scalenus medius & 2 & 39.5 & 2.65 & 40.3 & 30.5 & 0 & 0.76 & 0.18 & $\mathrm{C} 5$ & Point & -0.0043 & -0.1859 & 1.1250 & $\mathrm{R} 1$ & Surface & -0.0561 & -0.1608 & 1.0788 \\
\hline Scalenus medius & 3 & 50.0 & 2.65 & 51.0 & 34.0 & 0 & 2.38 & 0.44 & $\mathrm{C} 4$ & Point & -0.0004 & -0.1901 & 1.1400 & $\mathrm{R} 1$ & Surface & -0.0561 & -0.1608 & 1.0788 \\
\hline Scalenus medius & 4 & 40.3 & 2.65 & 41.1 & 56.8 & 0 & 0.90 & 0.21 & $\mathrm{C} 3$ & Point & 0.0026 & -0.1937 & 1.1537 & $\mathrm{R} 1$ & Surface & -0.0561 & -0.1608 & 1.0788 \\
\hline Scalenus medius & 5 & 100.0 & 2.65 & 102.1 & 19.0 & 0 & 0.90 & 0.08 & $\mathrm{C} 2$ & Point & 0.0037 & -0.1951 & 1.1648 & R1 & Surface & -0.0561 & -0.1608 & 1.0788 \\
\hline Scalenus medius & 6 & 69.0 & 2.65 & 70.4 & 57.0 & 0 & 2.38 & 0.32 & $\mathrm{C} 1$ & Point & -0.0033 & -0.1974 & 1.1852 & $\mathrm{R} 1$ & Surface & -0.0561 & -0.1608 & 1.0788 \\
\hline Scalenus posterior & 1 & 47.4 & 2.48 & 51.6 & 38.6 & 0 & 2.29 & 0.42 & C3 & Point & 0.0037 & -0.1929 & 1.1546 & R1 & Line & -0.0525 & -0.1523 & 1.0858 \\
\hline Semispinalis capitis & 1 & 142.5 & 3.06 & 125.6 & 80.7 & 10 & 1.55 & 0.16 & $\mathrm{~T} 6$ & Point & -0.0318 & -0.0626 & 1.0126 & Skull & Surface & 0.0179 & -0.1228 & 1.1930 \\
\hline Semispinalis capitis & 2 & 142.5 & 3.06 & 125.6 & 55.2 & 10 & 1.55 & 0.16 & T5 & Point & -0.0284 & -0.0743 & 1.0401 & Skull & Surface & 0.0179 & -0.1228 & 1.1930 \\
\hline Semispinalis capitis & 3 & 101.8 & 3.06 & 89.7 & 65.7 & 0 & 3.01 & 0.33 & $\mathrm{~T} 4$ & Point & -0.0267 & -0.0883 & 1.0592 & Skull & Surface & 0.0179 & -0.1228 & 1.1930 \\
\hline Semispinalis capitis & 4 & 96.0 & 3.06 & 84.6 & 34.5 & 0 & 2.35 & 0.26 & $\mathrm{~T} 3$ & Point & -0.0240 & -0.1041 & 1.0791 & Skull & Surface & 0.0179 & -0.1228 & 1.1930 \\
\hline Semispinalis capitis & 5 & 82.3 & 3.06 & 72.5 & 27.7 & 0 & 2.54 & 0.33 & $\mathrm{~T} 2$ & Point & -0.0231 & -0.1216 & 1.0978 & Skull & Surface & 0.0179 & -0.1228 & 1.1930 \\
\hline Semispinalis capitis & 6 & 82.3 & 3.06 & 72.5 & 17.7 & 0 & 2.54 & 0.33 & T1 & Line & -0.0179 & -0.1457 & 1.1126 & Skull & Surface & 0.0179 & -0.1228 & 1.1930 \\
\hline Semispinalis capitis & 7 & 48.8 & 3.06 & 43.0 & 39.8 & 0 & 1.97 & 0.43 & $\mathrm{C} 7$ & Point & -0.0131 & -0.1638 & 1.1160 & Skull & Surface & -0.0014 & -0.1333 & 1.1939 \\
\hline Semispinalis capitis & 8 & 48.8 & 3.06 & 43.0 & 37.3 & 0 & 1.97 & 0.43 & $\mathrm{C} 6$ & Point & -0.0046 & -0.1745 & 1.1192 & Skull & Surface & -0.0014 & -0.1333 & 1.1939 \\
\hline
\end{tabular}

Continued on next page 


\begin{tabular}{|c|c|c|c|c|c|c|c|c|c|c|c|c|c|c|c|c|c|c|}
\hline \multirow[t]{2}{*}{ Muscle } & \multirow[t]{2}{*}{ \# } & \multirow{2}{*}{$\begin{array}{l}\ell f \\
(\mathrm{~mm})\end{array}$} & \multirow{2}{*}{$\begin{array}{l}\ell^{s} \\
(\mu \mathrm{m})\end{array}$} & \multirow{2}{*}{$\begin{array}{l}\ell_{o}^{f} \\
(\mathrm{~mm})\end{array}$} & \multirow{2}{*}{$\begin{array}{l}\ell^{t} \\
(\mathrm{~mm})\end{array}$} & \multirow{2}{*}{$\begin{array}{l}\alpha \\
\text { (deg) }\end{array}$} & \multirow{2}{*}{$\begin{array}{l}\text { Mass } \\
\text { (g) }\end{array}$} & \multirow{2}{*}{$\begin{array}{l}\text { PCSA } \\
\left(\mathrm{cm}^{2}\right)\end{array}$} & \multirow{2}{*}{$\begin{array}{l}\text { Origin } \\
\text { (bone) }\end{array}$} & \multirow[t]{2}{*}{ Form } & \multicolumn{3}{|c|}{ Position (m) } & \multirow{2}{*}{$\begin{array}{l}\text { Insertion } \\
\text { (bone) }\end{array}$} & \multirow[t]{2}{*}{ Form } & \multicolumn{3}{|c|}{ Position (m) } \\
\hline & & & & & & & & & & & $\mathrm{x}$ & $\mathrm{y}$ & $\mathrm{z}$ & & & $\mathrm{x}$ & $\mathrm{y}$ & $\mathrm{z}$ \\
\hline Semispinalis capitis & 9 & 48.8 & 3.06 & 43.0 & 31.3 & 0 & 1.97 & 0.43 & C5 & Point & 0.0015 & -0.1841 & 1.1322 & Skull & Surface & -0.0014 & -0.1333 & 1.1939 \\
\hline Semispinalis capitis & 10 & 42.0 & 3.06 & 37.0 & 39.0 & 0 & 0.80 & 0.20 & $\mathrm{C} 4$ & Point & 0.0015 & -0.1886 & 1.1408 & Skull & Surface & -0.0014 & -0.1333 & 1.1939 \\
\hline Semispinalis cervicis & 1 & 69.5 & 3.91 & 48.0 & 42.0 & 0 & 1.71 & 0.34 & T5 & Point & -0.0279 & -0.0766 & 1.0436 & $\mathrm{C} 6$ & Point & 0.0172 & -0.1391 & 1.1273 \\
\hline Semispinalis cervicis & 2 & 73.3 & 3.91 & 50.7 & 61.7 & 0 & 0.81 & 0.15 & T5 & Point & -0.0279 & -0.0766 & 1.0436 & $\mathrm{C} 4$ & Point & 0.0188 & -0.1520 & 1.1391 \\
\hline Semispinalis cervicis & 4 & 41.5 & 3.91 & 28.7 & 62.5 & 0 & 0.20 & 0.07 & $\mathrm{~T} 4$ & Point & -0.0269 & -0.0882 & 1.0598 & $\mathrm{C} 4$ & Point & 0.0188 & -0.1520 & 1.1391 \\
\hline Semispinalis cervicis & 5 & 63.0 & 3.91 & 43.5 & 48.0 & 0 & 1.32 & 0.29 & $\mathrm{~T} 4$ & Point & -0.0269 & -0.0882 & 1.0598 & $\mathrm{C} 4$ & Point & 0.0188 & -0.1520 & 1.1391 \\
\hline Semispinalis cervicis & 6 & 67.5 & 3.91 & 46.6 & 65.5 & 0 & 0.42 & 0.09 & $\mathrm{~T} 4$ & Point & -0.0269 & -0.0882 & 1.0598 & $\mathrm{C} 2$ & Point & 0.0232 & -0.1576 & 1.1575 \\
\hline Semispinalis cervicis & 7 & 52.0 & 3.91 & 35.9 & 20.5 & 0 & 0.28 & 0.07 & T3 & Point & -0.0203 & -0.1072 & 1.0822 & C5 & Point & 0.0153 & -0.1457 & 1.1330 \\
\hline Semispinalis cervicis & 8 & 46.5 & 3.91 & 32.1 & 40.5 & 0 & 0.28 & 0.08 & $\mathrm{~T} 3$ & Point & -0.0203 & -0.1072 & 1.0822 & $\mathrm{C} 2$ & Point & 0.0232 & -0.1576 & 1.1575 \\
\hline Semispinalis cervicis & 9 & 46.5 & 3.91 & 32.1 & 13.5 & 0 & 0.59 & 0.17 & $\mathrm{~T} 2$ & Point & -0.0181 & -0.1262 & 1.0990 & $\mathrm{C} 4$ & Point & 0.0188 & -0.1520 & 1.1391 \\
\hline Semispinalis cervicis & 10 & 49.5 & 3.91 & 34.2 & 13.0 & 0 & 1.13 & 0.31 & $\mathrm{~T} 1$ & Point & -0.0161 & -0.1444 & 1.1124 & $\mathrm{C} 2$ & Point & 0.0232 & -0.1576 & 1.1575 \\
\hline Semispinalis thoracis ${ }^{h}$ & 1 & 55.4 & 3.80 & 39.3 & 96.6 & 0 & 0.68 & 0.16 & T9 & Point & -0.0312 & -0.0458 & 0.9278 & $\mathrm{~T} 2$ & Point & -0.0028 & -0.0934 & 1.0840 \\
\hline Semispinalis thoracis ${ }^{h}$ & 4 & 55.4 & 3.80 & 39.3 & 89.6 & 0 & 0.68 & 0.16 & $\mathrm{~T} 6$ & Point & -0.0319 & -0.0613 & 1.0143 & $\mathrm{C} 6$ & Point & 0.0169 & -0.1351 & 1.1316 \\
\hline Serratus anterior ${ }^{\mathrm{i}}$ & 1 & 143.2 & 2.40 & 161.3 & 19.3 & 0 & 7.91 & 0.46 & $\mathrm{R} 8$ & Line & -0.1464 & -0.1477 & 0.8547 & Scapula & Line & -0.1062 & -0.0588 & 0.9743 \\
\hline Serratus anterior ${ }^{\mathrm{i}}$ & 2 & 149.0 & 2.40 & 167.9 & 24.0 & 0 & 15.41 & 0.87 & $\mathrm{R} 7$ & Line & -0.1426 & -0.1679 & 0.8687 & Scapula & Line & -0.1133 & -0.0672 & 0.9776 \\
\hline Serratus anterior ${ }^{i}$ & 3 & 176.2 & 2.40 & 198.5 & 13.8 & 0 & 15.97 & 0.76 & $\mathrm{R} 6$ & Line & -0.1221 & -0.2017 & 0.8901 & Scapula & Line & -0.1209 & -0.0779 & 0.9856 \\
\hline Serratus anterior ${ }^{\mathrm{i}}$ & 4 & 146.3 & 2.40 & 164.9 & 38.7 & 0 & 13.17 & 0.76 & R5 & Line & -0.1176 & -0.2024 & 0.9201 & Scapula & Line & -0.1043 & -0.0559 & 0.9752 \\
\hline Serratus anterior $\mathrm{i}$ & 5 & 137.8 & 2.40 & 155.2 & 25.3 & 0 & 7.92 & 0.48 & R4 & Line & -0.1129 & -0.1958 & 0.9518 & Scapula & Line & -0.0996 & -0.0513 & 0.9892 \\
\hline Serratus anterior $\mathrm{i}^{\mathrm{i}}$ & 6 & 119.3 & 2.40 & 134.4 & 34.3 & 0 & 13.78 & 0.97 & R3 & Line & -0.1058 & -0.1963 & 0.9737 & Scapula & Line & -0.0967 & -0.0489 & 0.9962 \\
\hline Serratus anterior $\mathrm{i}$ & 7 & 86.7 & 2.40 & 97.7 & 38.3 & 0 & 20.71 & 2.01 & $\mathrm{R} 2$ & Line & -0.0929 & -0.1685 & 1.0238 & Scapula & Line & -0.0824 & -0.0500 & 1.0297 \\
\hline Serratus anterior ${ }^{\mathrm{i}}$ & 8 & 84.7 & 2.40 & 95.4 & 8.3 & 0 & 23.07 & 2.29 & $\mathrm{R} 1$ & Line & -0.0745 & -0.1606 & 1.0580 & Scapula & Line & -0.0495 & -0.0913 & 1.1055 \\
\hline Serratus posterior superior ${ }^{\mathrm{i}}$ & 1 & 39.5 & 3.59 & 29.7 & 77.5 & 0 & 0.83 & 0.26 & $\mathrm{~T} 1$ & Line & 0.0022 & -0.1053 & 1.1033 & R3 & Line & -0.0597 & -0.1020 & 1.0741 \\
\hline Serratus posterior superior ${ }^{\mathrm{i}}$ & 2 & 40.8 & 3.59 & 30.6 & 81.3 & 0 & 2.13 & 0.66 & $\mathrm{C} 7$ & Line & 0.0153 & -0.1163 & 1.1192 & $\mathrm{R} 2$ & Line & -0.0553 & -0.1274 & 1.0910 \\
\hline Serratus posterior superior ${ }^{\mathrm{i}}$ & 3 & 31.8 & 3.59 & 23.9 & 47.2 & 0 & 1.79 & 0.71 & $\mathrm{C} 6$ & Line & 0.0210 & -0.1326 & 1.1299 & $\mathrm{R} 1$ & Line & -0.0354 & -0.1532 & 1.1018 \\
\hline Spinalis thoracis ${ }^{b}, \mathrm{~h}$ & 1 & 25.0 & 3.55 & 19.0 & 110.0 & 0 & 0.02 & 0.01 & $\mathrm{~T} 12$ & Point & -0.0089 & -0.0438 & 0.8282 & $\mathrm{~T} 6$ & Point & -0.0102 & -0.0410 & 0.9749 \\
\hline Spinalis thoracis ${ }^{b, h}$ & 2 & 54.0 & 3.55 & 41.1 & 140.0 & 0 & 0.32 & 0.07 & $\mathrm{~T} 12$ & Point & -0.0089 & -0.0438 & 0.8282 & $\mathrm{~T} 5$ & Point & -0.0093 & -0.0494 & 1.0057 \\
\hline Spinalis thoracis $\mathrm{b}, \mathrm{h}$ & 3 & 41.3 & 3.55 & 31.4 & 186.0 & 0 & 0.53 & 0.16 & L1 & Point & -0.0075 & -0.0458 & 0.8100 & $\mathrm{~T} 4$ & Point & -0.0090 & -0.0604 & 1.0316 \\
\hline Spinalis thoracis $\mathrm{b}, \mathrm{h}$ & 4 & 47.0 & 3.55 & 35.8 & 219.5 & 0 & 0.78 & 0.21 & L1 & Point & -0.0075 & -0.0458 & 0.8100 & $\mathrm{~T} 4$ & Point & -0.0093 & -0.0690 & 1.0432 \\
\hline
\end{tabular}

Continued on next page 


\begin{tabular}{|c|c|c|c|c|c|c|c|c|c|c|c|c|c|c|c|c|c|c|}
\hline \multirow[t]{2}{*}{ Muscle } & \multirow[t]{2}{*}{$\#$} & \multirow{2}{*}{$\begin{array}{l}\ell f \\
(\mathrm{~mm})\end{array}$} & \multirow{2}{*}{$\begin{array}{l}\ell^{s} \\
(\mu \mathrm{m})\end{array}$} & \multirow{2}{*}{$\begin{array}{l}\ell_{o}^{f} \\
(\mathrm{~mm})\end{array}$} & \multirow{2}{*}{$\begin{array}{l}\ell^{t} \\
(\mathrm{~mm})\end{array}$} & \multirow{2}{*}{$\begin{array}{l}\alpha \\
(\operatorname{deg})\end{array}$} & \multirow{2}{*}{$\begin{array}{l}\text { Mass } \\
\text { (g) }\end{array}$} & \multirow{2}{*}{$\begin{array}{l}\text { PCSA } \\
\left(\mathrm{cm}^{2}\right)\end{array}$} & \multirow{2}{*}{$\begin{array}{l}\text { Origin } \\
\text { (bone) }\end{array}$} & \multirow[t]{2}{*}{ Form } & \multicolumn{3}{|c|}{ Position (m) } & \multirow{2}{*}{$\begin{array}{l}\text { Insertion } \\
\text { (bone) }\end{array}$} & & \multicolumn{3}{|c|}{ Position (m) } \\
\hline & & & & & & & & & & & $\mathrm{x}$ & $\mathrm{y}$ & $\mathrm{z}$ & & & $\mathrm{x}$ & $\mathrm{y}$ & $\mathrm{z}$ \\
\hline Spinalis thoracis ${ }^{\mathrm{b}, \mathrm{h}}$ & 5 & 53.0 & 3.55 & 40.3 & 224.0 & 0 & 1.02 & 0.24 & L1 & Point & -0.0075 & -0.0458 & 0.8100 & $\mathrm{~T} 3$ & Point & -0.0071 & -0.0768 & 1.0600 \\
\hline Spinalis thoracis ${ }^{\mathrm{b}, \mathrm{h}}$ & 6 & 20.0 & 3.55 & 15.2 & 101.5 & 0 & 0.04 & 0.02 & $\mathrm{~T} 12$ & Point & -0.0089 & -0.0438 & 0.8282 & $\mathrm{~T} 7$ & Point & -0.0072 & -0.0390 & 0.9401 \\
\hline Splenius capitis & 1 & 93.1 & 3.16 & 79.5 & 32.9 & 0 & 8.04 & 0.96 & $\mathrm{C} 7$ & Line & 0.0146 & -0.1246 & 1.1227 & Skull & Line & -0.0290 & -0.1798 & 1.2049 \\
\hline Splenius capitis & 2 & 67.7 & 3.16 & 57.8 & 28.3 & 0 & 6.55 & 1.07 & $\mathrm{C} 4$ & Line & 0.0222 & -0.1377 & 1.1462 & Skull & Line & -0.0273 & -0.1531 & 1.2102 \\
\hline Splenius cervicis & 1 & 77.5 & 3.55 & 58.9 & 79.5 & 0 & 2.89 & 0.46 & T3 & Line & -0.0022 & -0.0863 & 1.0722 & C3 & Point & 0.0029 & -0.1931 & 1.1540 \\
\hline Splenius cervicis & 2 & 83.0 & 3.55 & 63.1 & 75.0 & 0 & 5.17 & 0.77 & $\mathrm{~T} 2$ & Line & 0.0035 & -0.1022 & 1.0921 & $\mathrm{C} 1$ & Point & -0.0024 & -0.1959 & 1.1842 \\
\hline Sternocleidomastoid & 1 & 126.7 & 2.94 & 116.3 & 48.8 & 0 & 13.76 & 1.12 & Sternum & Surface & 0.0016 & -0.2165 & 1.0441 & Skull & Surface & -0.0279 & -0.1765 & 1.2033 \\
\hline Sternocleidomastoid & 2 & 118.7 & 2.94 & 109.0 & 87.3 & 0 & 12.00 & 1.04 & Sternum & Surface & -0.0054 & -0.2181 & 1.0430 & Skull & Line & -0.0301 & -0.1580 & 1.2119 \\
\hline Sternocleidomastoid & 3 & 80.0 & 2.94 & 73.0 & 61.0 & 0 & 10.30 & 1.33 & Clavicle & Surface & -0.0318 & -0.2050 & 1.0725 & Skull & Line & -0.0279 & -0.1765 & 1.2033 \\
\hline Sternocleidomastoid & 4 & 112.8 & 2.94 & 103.6 & 55.2 & 0 & 3.15 & 0.29 & Clavicle & Surface & -0.0437 & -0.1999 & 1.0813 & Skull & Line & -0.0192 & -0.1351 & 1.2101 \\
\hline Sternohyoid & 1 & 85.5 & 2.71 & 85.3 & 5.5 & 0 & 1.43 & 0.16 & Sternum & Line & 0.0014 & -0.2014 & 1.0514 & Hyoid & Line & 0.0119 & -0.2303 & 1.1417 \\
\hline Sternohyoid & 2 & 79.0 & 2.71 & 78.8 & 3.0 & 0 & 1.40 & 0.17 & Clavicle & Line & -0.0111 & -0.1984 & 1.0609 & Hyoid & Line & 0.0057 & -0.2261 & 1.1402 \\
\hline Sternothyroid & 1 & 60.0 & 2.10 & 77.0 & 6.5 & 0 & 0.26 & 0.03 & $\mathrm{R} 1$ & Line & -0.0298 & -0.1930 & 1.0520 & Thyroid & Line & 0.0032 & -0.2047 & 1.1218 \\
\hline Sternothyroid & 2 & 50.2 & 2.10 & 64.4 & 10.8 & 0 & 0.39 & 0.06 & Sternum & Line & 0.0014 & -0.2014 & 1.0514 & Thyroid & Line & 0.0064 & -0.2123 & 1.1155 \\
\hline Subclavious & 1 & 36.0 & 2.30 & 42.3 & 54.0 & 0 & 1.51 & 0.34 & $\mathrm{R} 1$ & Surface & -0.0445 & -0.1920 & 1.0522 & Clavicle & Surface & -0.0856 & -0.1735 & 1.1053 \\
\hline Subcostales & 1 & 23.0 & 2.33 & 26.7 & 36.5 & 0 & 0.17 & 0.06 & R11 & Line & -0.0371 & -0.0657 & 0.8831 & R9 & Line & -0.0706 & -0.0523 & 0.9301 \\
\hline Subcostales & 2 & 23.5 & 2.33 & 27.2 & 28.5 & 0 & 0.45 & 0.16 & R10 & Line & -0.0374 & -0.0586 & 0.9180 & $\mathrm{R} 8$ & Line & -0.0680 & -0.0554 & 0.9611 \\
\hline Subcostales & 3 & 26.0 & 2.33 & 30.1 & 17.5 & 0 & 0.72 & 0.23 & R9 & Line & -0.0415 & -0.0563 & 0.9480 & $\mathrm{R} 7$ & Line & -0.0579 & -0.0600 & 0.9892 \\
\hline Subcostales & 4 & 23.5 & 2.33 & 27.2 & 15.5 & 0 & 0.31 & 0.11 & $\mathrm{R} 8$ & Line & -0.0434 & -0.0583 & 0.9741 & R6 & Line & -0.0505 & -0.0691 & 1.0139 \\
\hline Subcostales & 5 & 26.3 & 2.33 & 30.4 & 12.8 & 0 & 0.41 & 0.13 & $\mathrm{R} 7$ & Line & -0.0424 & -0.0658 & 1.0000 & R5 & Line & -0.0544 & -0.0800 & 1.0350 \\
\hline Subcostales & 6 & 10.0 & 2.33 & 11.6 & 10.0 & 0 & 0.05 & 0.04 & $\mathrm{R} 6$ & Line & -0.0463 & -0.0732 & 1.0201 & R5 & Line & -0.0501 & -0.0820 & 1.0387 \\
\hline Subcostales & 7 & 21.8 & 2.33 & 25.2 & 22.8 & 0 & 0.81 & 0.30 & R5 & Line & -0.0510 & -0.0816 & 1.0382 & R3 & Line & -0.0572 & -0.1105 & 1.0710 \\
\hline Subcostales & 8 & 23.3 & 2.33 & 27.0 & 23.3 & 0 & 0.33 & 0.12 & $\mathrm{R} 4$ & Line & -0.0453 & -0.0949 & 1.0580 & $\mathrm{R} 2$ & Line & -0.0504 & -0.1322 & 1.0872 \\
\hline Thyrohyoid & 1 & 24.8 & 2.46 & 27.1 & 4.3 & 0 & 0.95 & 0.33 & Thyroid & Line & 0.0038 & -0.2060 & 1.1202 & Hyoid & Line & 0.0028 & -0.2192 & 1.1416 \\
\hline Transversus thoracis & 1 & 24.7 & 2.90 & 23.0 & 55.3 & 0 & 0.39 & 0.16 & $\mathrm{R} 2$ & Line & -0.0577 & -0.2088 & 1.0158 & Sternum & Line & -0.0126 & -0.2298 & 0.9535 \\
\hline Transversus thoracis & 2 & 25.8 & 2.90 & 24.0 & 36.3 & 0 & 0.38 & 0.15 & R3 & Line & -0.0632 & -0.2248 & 0.9769 & Sternum & Line & -0.0103 & -0.2289 & 0.9431 \\
\hline Transversus thoracis & 3 & 24.7 & 2.90 & 23.0 & 52.3 & 0 & 0.47 & 0.19 & $\mathrm{R} 4$ & Line & -0.0768 & -0.2230 & 0.9498 & Sternum & Line & -0.0027 & -0.2276 & 0.9286 \\
\hline Transversus thoracis & 4 & 25.3 & 2.90 & 23.5 & 34.3 & 0 & 0.38 & 0.15 & R5 & Line & -0.0600 & -0.2350 & 0.9300 & Sternum & Line & -0.0021 & -0.2268 & 0.9171 \\
\hline
\end{tabular}

Continued on next page

\section{$\omega$}




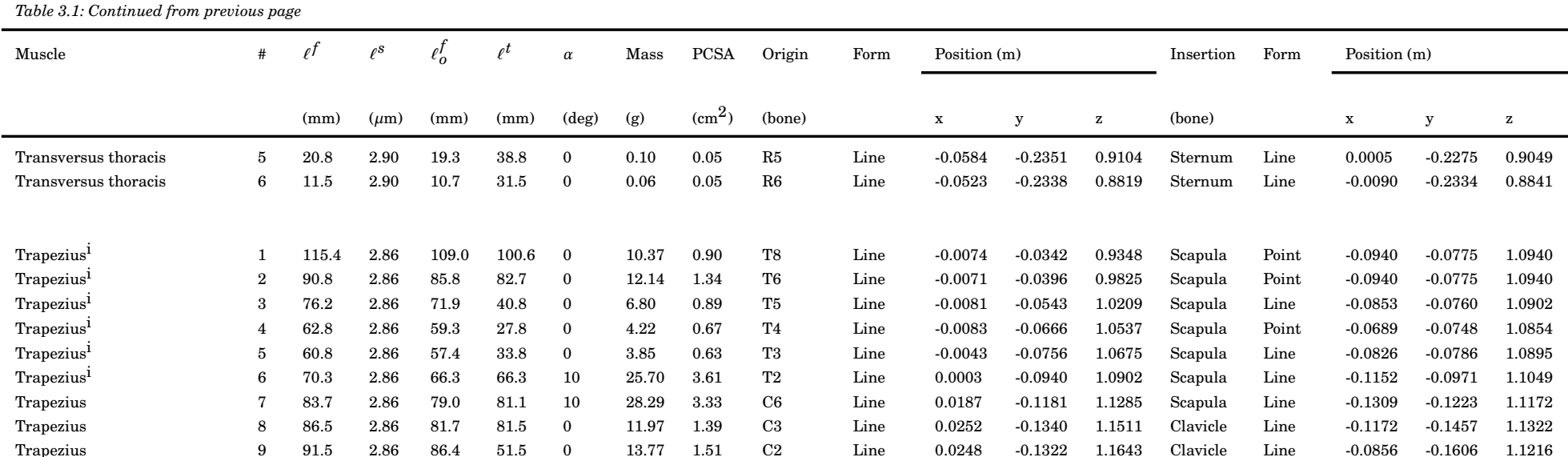

${ }^{a}$ Unless noted otherwise, muscle elements had tendon-muscle fiber-tendon architecture as illustrated in Fig. 3.2 .

${ }^{\mathrm{b}}$ Muscle fibers partially spanned the length of the tendon (see Fig. 3.2p).

${ }^{\mathrm{c}}$ Muscle fibers spanned the full length of the tendon (see Fig. 3.2 . ).

${ }^{d}$ Numbering the elements of the multifidus muscle starts from sixteen. The first fifteen elements belonged to the lumbar region of the spine and were described in our previous study 84

${ }^{e}$ We measured intercostales interni and intercostales externi muscles only at the following levels: between ribs 1 and 2, 5 and 6, and 9 and 10 .

${ }^{\mathrm{f}}$ We measured the levatores costarum longi only at the upper and lower rib levels.

${ }^{\mathrm{g} W e}$ could not get good samples for the sarcomere length measurements from the rectus capitis lateralis muscle. Therefore, we assumed a mean sarcomere length of $2.63 \mu \mathrm{m}$ (calculated mean sarcomere length for the rectus capitis anterior muscle) for this muscle.

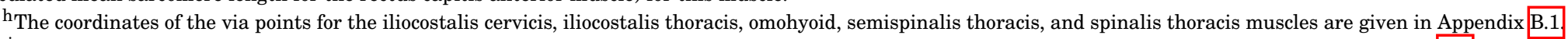

${ }^{\mathrm{i}}$ The mathematical definitions of the wrapping surfaces for the trapezius, serratus anterior, and serratus posterior superior muscles are given in Appendix B.2. 


\subsection{Discussion}

The aim of the present study was to obtain a complete and coherent musculoskeletal dataset for modeling the thoracic and cervical regions of the human spine. For this purpose, we dissected several muscles from a single spine, measured the positions of their attachments at the bones, and obtained three-dimensional geometry of the bones (in the form of STL files). We chose to divide the muscles into a number of muscle-tendon elements to represent their function better in computer models. For every muscle element, we additionally measured the morphological parameters consisting of the fiber length, tendon length, sarcomere length, optimal fiber length, pennation angle, mass, and PCSA. These architectural parameters will enable better simulation of the muscle mechanics and hence will improve the muscle and joint force estimations in such models [35, 74, 91]. For example, Carbone et al. (2016) [43] investigated the sensitivity of their gait model against potential errors in morphological parameters for several muscles. They reported tendon slack length to be the most sensitive parameter, subsequently followed by maximal isometric muscle force, optimal muscle fiber length, and nominal pennation angle.

Morphological parameters of the neck muscles measured in this study are compared with similar anatomical studies in Table 3.2. The PCSAs of the individual elements of a muscle were summed, and a mean value of their optimal fiber lengths was calculated for comparison. PCSAs measured in this study fit well with the range of data reported in the literature. Furthermore, the mean sarcomere lengths of some muscles were higher than those reported in [52] and [38]. The mean sarcomere lengths of up to $3.91 \mu \mathrm{m}$ were measured for the cervicis and capitis muscles which originated from the thoracic sixth vertebra and above. Moreover, the mean optimal fiber lengths were systematically lower compared to [38], but very similar to the data in [52]. Similarly, the comparison of the muscle fiber lengths indicated lower fiber lengths in this study compared to [38]. We attributed this disparity to the differences between the heights of the cadavers measured (154 cm in this study and $171 \mathrm{~cm}$ in [38]). We calculated optimal fiber lengths by dividing measured mean fiber lengths by the mean sarcomere lengths of the muscles and multiplying with $2.7 \mu \mathrm{m}$ (assumed optimal sarcomere length for skeletal muscles [61]). Therefore, the higher sarcomere lengths (in general) and the lower fiber lengths measured in this study explain the discrepancy between the optimal fiber lengths.

Several limitations affect the data presented in this study. Firstly, the architecture of the neck muscles may be affected due to scoliosis around this region [92]. Secondly, we dissected the muscles only from one side of the cadaver. One then needs to assume about the skeletal geometry and muscle architecture for the other side when building a model. It is a common practice to create a left-right symmetrical musculoskeletal model, however, the validity of such assumptions may be questionable [93]. Thirdly, measured architectural parameters 


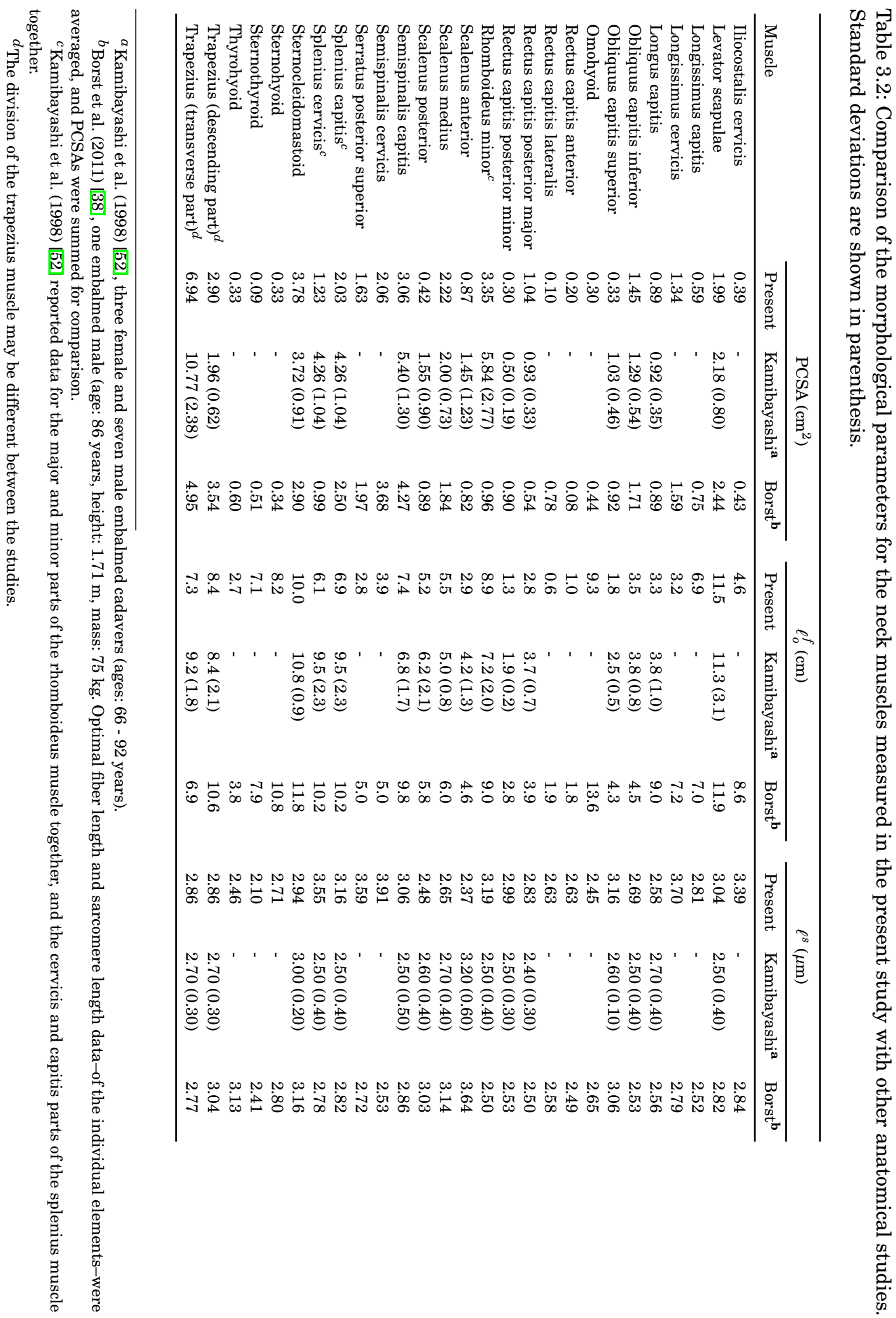


of the muscles may deviate from in vivo. A mean shrinkage of $2 \%$ in muscle fiber lengths was reported when the muscles were fixed in isolation [79]. This suggests higher sarcomere and fiber lengths when measured in vivo and thus likely to cause slight changes in estimated optimal fiber lengths and physiological cross-sectional areas. Fourthly, this dataset was obtained from an older-adult male. Earlier studies discussed that isometric muscle strength declines with aging [94]. Hakkinen et al. (1991) [95] suggested that the decline in isometric strength with aging may be associated with the decrease in the cross-sectional area of the muscle. Thus, we advise taking into account age-related changes in muscle morphology by adjusting the parameters of the Hill-type musculo-tendon models when this dataset is used to study younger populations [91]. For example, the isometric strengths of the muscles can be increased when studying young individuals. Moreover, some studies reported on the gender-related differences in muscle morphology [96-98]. They asserted that men, in general, have greater upper and lower body strength compared to women. Therefore, when modeling female subjects (of similar age as the cadaver in this study), the isometric strengths of the muscles reported in the present study should be adjusted accordingly. Fifthly, we simplified the otherwise complex functions of muscles by dividing them into several muscle-tendon elements. This approach was a choice of modeling as such and required some assumptions. The details and underlying assumptions of this approach were described elsewhere [80]. Sixthly, the subcostales muscle was partially damaged on the right side during the resection of the intercostales muscle. For this reason, we collected the data for this muscle from the left side of the cadaver. Similarly, rectus capitis muscles (anterior and lateralis) were also measured on the left side as they were damaged during the resection of the neighboring muscles. Seventhly, intercostales muscles were measured only at three levels, between ribs 1 and 2, 5 and 6, and 9 and 10. In addition, levatores costarum longi (elements 2,10 , and 12) were measured only at the upper and lower rib levels. It may be reasonable to assume that measured parameters do not vary a lot between different levels for these muscles. Thus, interpolating or averaging of the reported data regarding these muscles can be used when modeling other levels. Lastly, measuring the coordinates of the attachments was impossible for the subcostales and transversus thoracis muscles as they were located inside the ribcage. Therefore, we identified their attachments at the bones and later visually defined their lines-of-action based on the pictures of the corresponding regions and anatomical illustrations given in [99] and [100].

The resection of the muscles around the cervical region of the spine revealed a notable anatomical variability in terms of the muscle attachments with the bones. A good example for this is the scalenus muscle group. Standard anatomy books such as [100] describe the origin attachments of the scalenus anterior at the cervical third (C3) to sixth vertebrae (C6) and scalenus posterior at the $\mathrm{C} 4-\mathrm{C} 6$. Our measurements yet indicated the attachments at the $\mathrm{C} 5-\mathrm{C} 7$ and at the $\mathrm{C} 3$ vertebrae for these muscles, respectively. Such issues of anatomical variability are mentioned in Gray's book, [100]. We found similar variations for certain 
lumbar muscles in this cadaver as discussed in our earlier study [84]. This leads to the conclusion that certain care should be taken if a subject specific model is developed from a generic model or dataset.

Ward et al. (2009) [73] discussed that the lumbar multifidus muscle acts as a stabilizer of the lumbar spine by producing considerable forces due to its architectural design associated with a high cross-sectional area and a low fiber length to muscle length ratio. Bojadsen et al. (2000) [101] and Cornwall et al. (2011) [102] mentioned on the architectural differences of the multifidus muscle in the lumbar and thoracic regions of the spine. Bojadsen et al. (2000) [101] noted a pronounced increase in the tendinous tissue of this muscle in the thoracic region compared to the lumbar region. Furthermore, they postulated that the thoracic multifidus muscle (located on the antagonistic side) is exposed to large strains during the axial rotation of the spine and this seems related to the increase in tendinous tissue, which is much more resistant to deformation than the muscle tissue, in the upper thoracic spine. The morphology of the other deeper back muscles such as rotatores, levatores costarum, and semispinalis muscles has been investigated before, but only qualitatively [102, 103]. In this study, we also measured the transversospinal muscle group including levatores costarum and spinalis thoracis muscles and report new data for them. One common feature of these muscles (rotatores, levatores costarum, spinalis thoracis, and most elements of the multifidus) was that they were mostly tendinous in architecture, and their tendons ran from origin to insertion (musculo-tendon length being equal to tendon length) and were mixed with the muscle fibers. These muscle fibers either spanned the total length of the tendon or partly spanned (both of which are indicated in Table 3.1). The second common feature was that they had relatively small PCSAs implying that the active muscle force contribution will be small in these muscles. Such muscles can be modeled in several ways in computer models, for example as ligaments. In that case, the stiffness of the ligament, i.e. tendon, needs to be known. The stiffness can be calculated from the cross-sectional area, Young's modulus, and length of the tendon [104]. For this purpose, we additionally provide the tendon cross-sectional areas for these muscle elements. Due to their architectural design, we think that these muscles stabilize the spine at different regions preventing our joints from injury during excessive movements. Next to stabilizing function, they may also be responsible for protection by preventing overstretch of the muscle fibers. Further research is planned on the best way of modeling the actions of these muscles in musculoskeletal models of the spine.

In our previous study, we presented an anatomical dataset for modeling the lumbar region of the spine [84]. The dataset reported in this study was obtained from the same cadaver, therefore, it complements our earlier study and make a complete musculoskeletal dataset for modeling the entire human spine. Because the complete dataset was obtained from a single cadaver, it is coherent and eliminates the uncertainties that come with combining musculoskeletal data from different specimens [38, 60]. All the bones and muscles obtained 

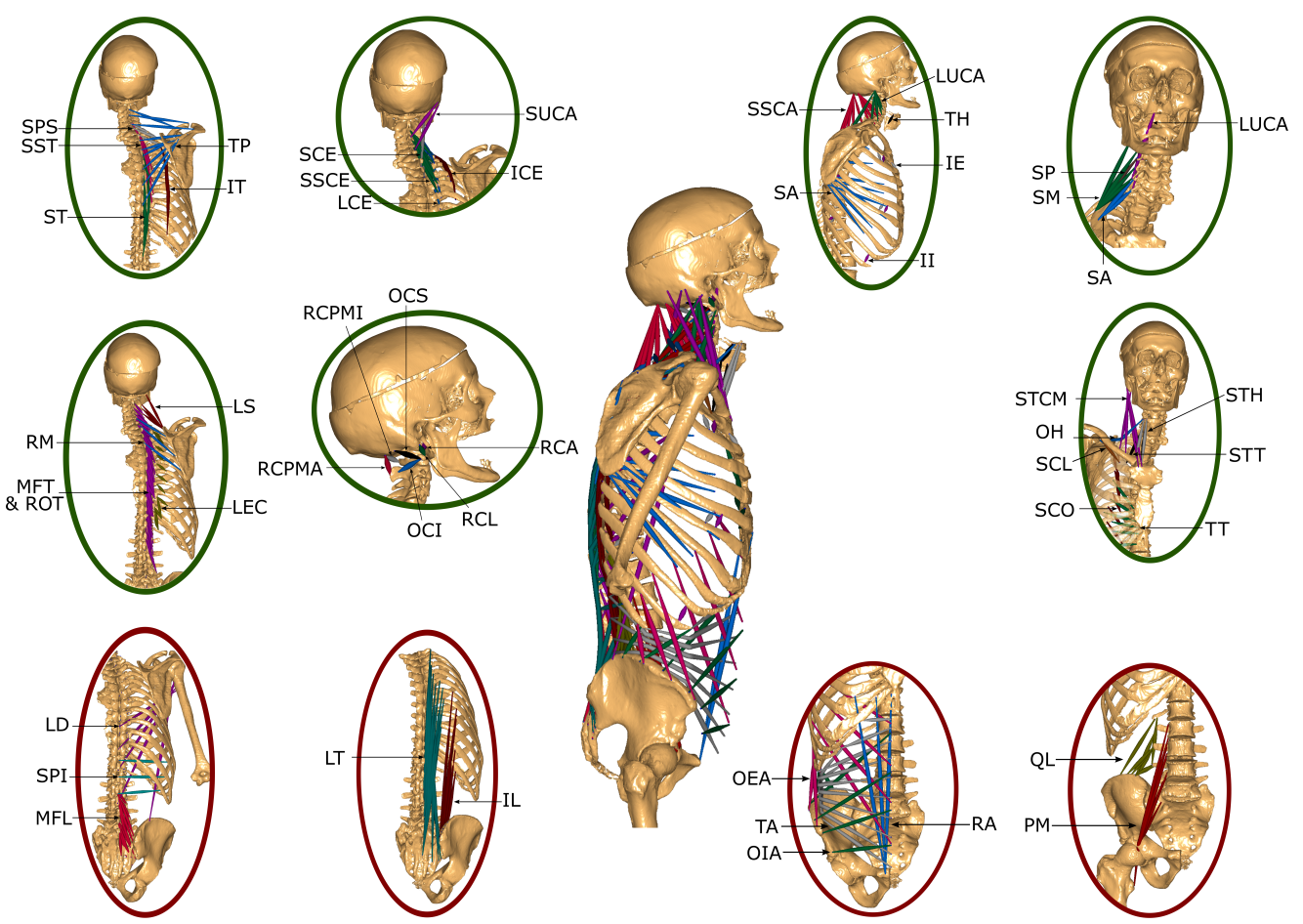

Figure 3.4: The muscles measured in the complete anatomical dataset are highlighted. The muscles presented in this study are emphasized with green circles, whereas the muscles reported in our previous study with red circles [84]. See Appendix B.4 for the abbreviations used for the muscles.

for the complete dataset are illustrated in Fig. 3.4 We hope that the complete dataset will further our understanding of the functioning of the healthy and pathological spine. All the data reported in this paper is shared with the scientific community through

https://www.utwente.nl/en/et/bw/research/projects/twentespinemodel.

\section{Conflict of interest}

None of the authors have any financial or personal relationships with other people or organization that could inappropriately influence their work.

\section{Acknowledgments}

We acknowledge the financial supports by Fonds NutsOhra and the European Research Council 'the BioMechTools project'. Furthermore, we like to thank several colleagues for their help on this work, Vincenzo Carbone and Kenan Niu for useful discussions on musculoskeletal modeling and motion capture systems, Alper Denasi for giving feedback on the 
manuscript, Frans Segerink for providing a laser and giving insights into laser diffraction technique, Andre Sprengers for his help during imaging sessions, and the Radiology Department and the Anatomy Department of the Radboud University Medical Center for their hospitality during the experiment. Finally, we thank Huub Maas from VU University of Amsterdam for sharing his expertise on sarcomere length measurements. 


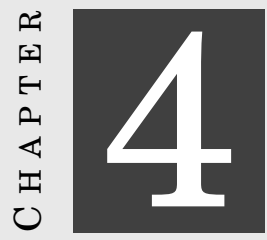

\title{
TWENTE SPINE MODEL: A THOROUGH INVESTIGATION OF THE SPINAL LOADS IN A COMPLETE AND COHERENT MUSCULOSKELETAL MODEL OF THE HUMAN SPINE
}

\begin{abstract}
Although in vivo spinal loads have been previously measured, existing data are limited to certain lumbar and thoracic levels. A detailed investigation of spinal loads would assist with injury prevention and implant design but is unavailable. In this study, we developed a complete and coherent musculoskeletal model of the entire human spine and studied the intervertebral disc compression forces for physiological movements on three anatomical planes. This model incorporates the individual vertebrae at the cervical, thoracic, and lumbar regions, a flexible ribcage, and complete muscle anatomy. Intradiscal pressures were estimated from predicted compressive forces, and these were generally in close agreement with previously measured data. We found that compressive forces at the trunk discs increased during trunk lateral bending and axial rotation of the trunk. During flexion, compressive forces increased in the thoracolumbar and lumbar regions and slightly decreased at the middle thoracic discs. In extension, the forces generally decreased at the thoracolumbar and lumbar discs whereas they slightly increased at the upper and middle thoracic discs. Furthermore, similar to a previous biomechanical model of the cervical spine, our model predicted increased compression forces in neck flexion, lateral bending, and axial rotation, and decreased forces in neck extension.
\end{abstract}

This chapter has been published as: Riza Bayoglu, Pavel. E. Galibarov, Nico Verdonschot, Bart Koopman, Jasper Homminga, "Twente Spine Model: A thorough investigation of the spinal loads in a complete and coherent musculoskeletal model of the human spine”, Medical Engineering and Physics, $2019,68 \mathrm{C}, 35-45$. 


\subsection{Introduction}

Spinal loads have been previously quantified mostly through intervertebral disc pressures or instrumented implants. Several authors have measured in vivo pressures at the lumbar and thoracic discs [3-8]. Differently, resultant forces and moments on lumbar telemetered implants were measured [10]. Although detailed knowledge of spinal loads is available, it is limited to certain levels in the lumbar and thoracic regions due to ethical issues. Also, this data has been valuable for estimating the compressive forces at the intervertebral discs but less so for other forces such as shear forces at the discs or muscle forces. A comprehensive assessment of spinal loads would assist with injury prevention and implant design but is yet unavailable.

Several researchers have developed validated models to explore the nature of the loads within the spine [26-28, 30, 33, 34, 37]. These models have evolved in complexity for representing generic or subject-specific musculoskeletal systems. Compared to generic models, subject-specific models offer more realistic representations of the skeletal and muscular anatomy [38]. The majority of these models included only a certain region of the spine and assumed a single, lumped, rigid body representation of the thorax or neck. The effects of modeling the thorax this way instead of a flexible structure when estimating the lumbar loads were previously studied for flexion [40]. They found moderately lower compressive disc forces but remarkably altered muscle forces. It remains unclear how such modeling approaches would affect force predictions when other movements are investigated. The majority of the previous models have relied on anatomical illustrations for defining muscle lines-of-action and lacked deeper spinal muscles which function as joint stabilizers. Recent studies developed methods for implementing moment arms and cross-sectional areas of major trunk muscles from medical images [33]. Obtaining muscle origins and insertions at the bones precisely is still difficult with current imaging modalities particularly for smaller and deeper muscles [42]. Furthermore, muscle parameters that are needed and influential in the model (physiological cross-sectional area (PCSA) for example, [43]) can not be reliably determined from two-dimensional medical imaging [42, 44]. In essence, there is also a need for a complete and coherent musculoskeletal model of the entire human spine, which is likely to yield realistic spinal loads [38].

Recently we published a musculoskeletal dataset which was measured from one single human body [84, 105]. In this dataset, the coordinates of muscle attachments, architectural parameters of 321 muscle-tendon elements, and the spinal geometry were reported. Furthermore, new data were obtained for several muscles in the thoracic and lumbar regions. The objective of this study is now to investigate the compressive forces at the intervertebral discs throughout the spine during basic trunk and neck movements. For this, we developed and used a complete and coherent musculoskeletal model of the entire human spine (based 
on the previously published dataset) and validated it by comparing calculated spinal loads to in vivo measurements. Subsequently, we used this model to determine the spinal loads at every spinal level, which until now could not be determined. This model represents a subject-specific musculoskeletal system and has several particular features, such as incorporation of the individual vertebrae at the cervical, thoracic, and lumbar regions, flexible ribcage, and comprehensive muscular anatomy.

\subsection{Materials and methods}

\subsubsection{Development of the model}

We developed a musculoskeletal model for the entire human spine in AnyBody Modeling System. This model was based on a coherent anatomical dataset measured from one embalmed cadaver (male, 79 years-old, height: $154 \mathrm{~cm}$, mass: $51 \mathrm{~kg}$ ) [84, 105]. In this model, the bones were idealized as rigid body segments and the muscle-tendon elements as force actuators. The model included 60 segments (vertebrae, ribs, skull, sternum, hyoid, thyrohyoid, clavicles, scapulas, humeri, sacrum, and pelvis) and had $193 \mathrm{DoF}$ (Fig. 4.1). The lumped masses and the centers-of-mass (CoM) of the body segments were defined based on previous reports [34, 106-108]. The hyoid, thyrohyoid, clavicle, scapula, and the humerus segments were included without mass properties as these only functioned as attachment sites for muscles that play a role in lateral bending and axial rotation. Our model does not explicitly incorporate the lower extremity and the shoulder-arm complex nor the body segments and muscles located in these extremities. The arms and other segments are present for further modeling. External forces and moments were applied at the third thoracic vertebrae to model the loads that would be exerted by the shoulder-arm complex [109]. We set the mass moments-of-inertia for all the segments to zero based on the fact that their rotational velocities and accelerations are negligible during basic trunk movements [26].

We utilized previously reconstructed stereolithography (STL) files of the bones [84, 105]. First, spinal geometry was made left-right symmetrical. For this, we established a local reference frame for each vertebra and mirrored its right side in the local sagittal plane [110] in Solidworks 2016 (Fig. 4.1). Similarly, the other bones in the skeletal system also were made left-right symmetrical. Next, the positions and the orientations of the individual segments were adjusted to reconstruct the spinal curvature at the supine position. Finally, muscle attachment sites reported in the global reference frame were expressed in corresponding vertebral frames. 


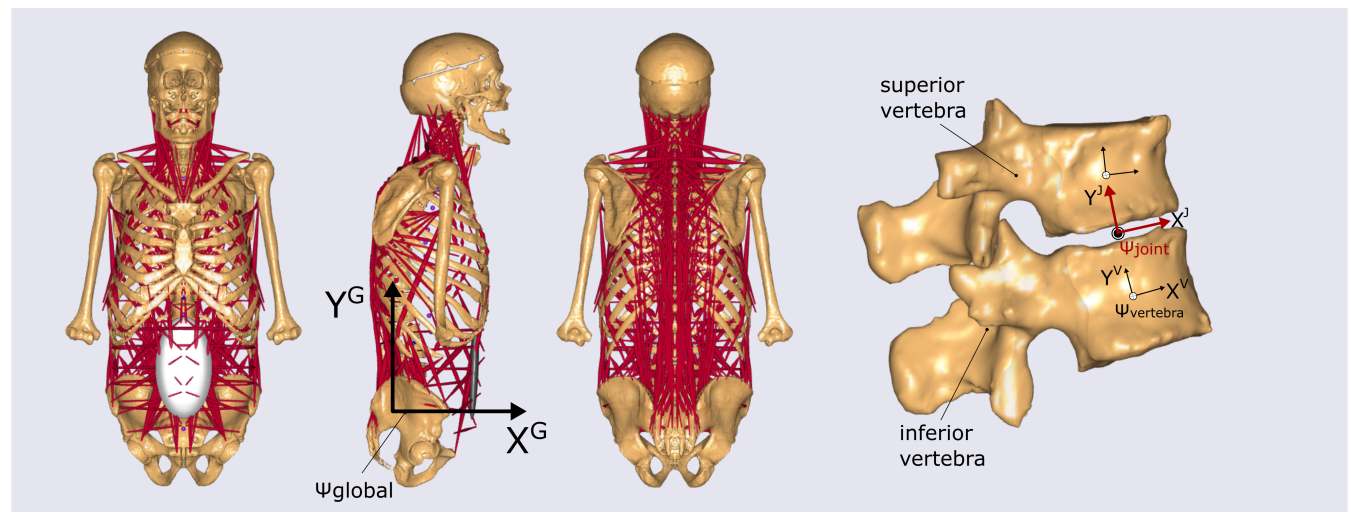

Figure 4.1: The musculoskeletal model developed in this study, bones are displayed in beige and muscle-tendon elements in red. The reference frame shown in the sagittal view is the global reference frame ( $\left.\psi_{\text {global }}\right)$. The vertebral $\left(\psi_{\text {vertebra }}\right)$ and intervertebral disc joint reference frames $\left(\psi_{\text {joint }}\right)$ are illustrated for one motion segment. The origin of the vertebral reference frame is located at the centroid of the vertebral body, the $y$-axis passes through the geometric centers of the inferior and superior end-plates, and the $z$-axis is parallel to the line connecting similar landmarks on the left and right pedicles (orthogonal to the sagittal plane). The orientation of the intervertebral joint reference frame is defined as the average of its corresponding superior and inferior vertebra reference frame. The invertebral disc inclinations were calculated as the average angles of the superior and inferior vertebrae on the sagittal plane. The intervertebral compression force is calculated along the $y$-axis of the joint reference frame (perpendicular to the disc inclined plane) while shear is the force component on the $x z$ plane (parallel to the disc inclined plane).

Our model relied on inverse dynamics principles for predicting muscle activations where an optimization function was employed. Similar to previous models [33, 34], a third degree, polynomial muscle recruitment criterion was implemented [25].

\subsubsection{Muscular anatomy}

In total, 351 muscle-tendon elements (per body side) were included for 49 muscles. The isometric muscle strength was calculated by multiplying its PCSA by the specific muscle tension $\left(100 \mathrm{~N} / \mathrm{cm}^{2}\right)$. Because the data for the intercostales and the levatores costarum longi muscles along the entire ribcage was lacking in the original dataset, we introduced additional elements for these muscles at the missing levels. The morphological parameters for these elements were taken as the averages of the measured data. We also incorporated the abdominal muscle implementation from the human body model of the AnyBody Managed Model Repository. The changes in the length of the transversus abdominis muscle elements and the abdominal volume resulted in a change of intra-abdominal pressure, hence force in these muscles. All the muscles included in this study are listed in Table 4.1 . 
Table 4.1: Muscles included in the model.

\begin{tabular}{ll}
\hline Region & Muscle \\
\hline Cervical spine & $\begin{array}{l}\text { Iliocostalis cervicis, Levator scapulae, Longissimus capitis, Longissimus cervicis } \\
\text { Longus capitis, Obliquus capitis inferior, Obliquus capitis superior, Omohyoid } \\
\text { Rectus capitis anterior, Rectus capitis lateralis, Rectus capitis posterior major } \\
\text { Rectus capitis posterior minor, Scalenus anterior, Scalenus medius, Scalenus posterior } \\
\text { Semispinalis capitis, Semispinalis cervicis, Splenius capitis, Splenius cervicis } \\
\text { Sternocleidomastoid, Sternohyoid, Sternothyroid, Thyrohyoid }\end{array}$ \\
Thoracic spine & $\begin{array}{l}\text { Iliocostalis thoracis, Rhomboideus major, Rhomboideus minor, Rotatores } \\
\text { Semispinalis thoracis, Serratus anterior, Serratus posterior superior, Spinalis thoracis }\end{array}$ \\
& Subclavious, Trapezius \\
& Intercostales externi, Intercostales interni, Levatores costarum \\
Ribcage & Subcostales, Transversus thoracis \\
Lumbar spine & Iliocostalis lumborum, Latissimus dorsi, Longissimus thoracis, Multifidus \\
& Obliquus externus abdominis, Obliquus internus abdominis, Psoas major \\
& $\begin{array}{l}\text { Quadratus lumborum, Rectus abdominis, Serratus posterior inferior } \\
\text { Transversus abdominis }\end{array}$ \\
\hline
\end{tabular}

\subsubsection{Joints}

The intervertebral articulations were modeled as spherical joints. The locations of lumbar and cervical intervertebral joints were based on previous reports [111, 112]. In the thoracic spine, the geometric centers of the intervertebral discs were used. The costosternal articulations between the costal cartilages and the sternum were defined as six DoF joints. The costotransverse and the costovertebral joints were simplified as single-compound revolute joints which allowed rotation about the ribs' cervical axes [34, 113]. We defined the locations and reference frame axes of the aforementioned joints in line with previous studies [22, 114, 115]. Sternoclavicular, acromioclavicular, glenohumeral, and the articulations of the hyoid and the thyroid bones with the $\mathrm{C} 3$ and $\mathrm{C} 4$ vertebrae, respectively, were modeled as six DoF joints. No movement was prescribed at these joints implying that they were rigidly connected to the segments they articulated with.

The elastic behavior of the intervertebral joints was modeled as a lumped stiffness induced by the ligaments, discs, and the facet joints using linear torsion springs in three directions. In addition, the tension/compression stiffnesses for the costovertebral articulations and the tension/compression, shear, and the bending stiffnesses for the costotransverse articulations were defined using the stiffness data reported in previous studies (Table 4.2).

Soft constraints were applied for prescribing the movement at the compound-costotransverse and costovertebral joints and the costosternal joints. These constraints allowed minimal deviation from the idealized joints by minimizing the total elastic energy of deformation and determined the movement at these joints. This approach facilitated defining the oth- 
Table 4.2: The stiffness data adapted from the literature 23.117 .120$.

\begin{tabular}{|c|c|c|c|c|c|c|c|}
\hline Joints & $\begin{array}{l}\text { Flexion } \\
{[\mathrm{Nm} / \mathrm{deg}]}\end{array}$ & $\begin{array}{l}\text { Extension } \\
{[\mathrm{Nm} / \mathrm{deg}]}\end{array}$ & $\begin{array}{l}\text { Lateral } \\
\text { bending } \\
{[\mathrm{Nm} / \mathrm{deg}]}\end{array}$ & $\begin{array}{l}\text { Axial } \\
\text { rotation } \\
{[\mathrm{Nm} / \mathrm{deg}]}\end{array}$ & $\begin{array}{l}\text { Tension } \\
{[\mathrm{kN} / \mathrm{m}]}\end{array}$ & $\begin{array}{l}\text { Compression } \\
{[\mathrm{kN} / \mathrm{m}]}\end{array}$ & $\begin{array}{l}\text { Shear } \\
{[\mathrm{kN} / \mathrm{m}]}\end{array}$ \\
\hline \multicolumn{8}{|l|}{ Intervertebral } \\
\hline $\mathrm{C} 0 / \mathrm{C} 1$ & 0.04 & 0.02 & 0.09 & 0.06 & fixed & fixed & fixed \\
\hline $\mathrm{C} 1 / \mathrm{C} 2$ & 0.06 & 0.05 & 0.09 & 0.07 & fixed & fixed & fixed \\
\hline $\mathrm{C} 2-\mathrm{T} 1$ & 0.43 & 0.73 & 0.68 & 1.16 & fixed & fixed & fixed \\
\hline $\mathrm{T} 1-\mathrm{T} 12$ & 2.65 & 3.25 & 2.27 & 2.60 & fixed & fixed & fixed \\
\hline $\mathrm{T} 12-\mathrm{S} 1$ & 1.80 & 2.60 & 2.30 & 6.90 & fixed & fixed & fixed \\
\hline Costovertebral & fixed & fixed & fixed & fixed & 4.9 & 49 & fixed \\
\hline Costotransverse & 0.12 & 0.12 & 0.12 & 0.17 & 4.9 & 49 & 123 \\
\hline
\end{tabular}

erwise mechanically over-constrained nature of the costosternal joints in a kinematically determinant way [116].

\subsubsection{Comparison with in vivo intradiscal pressures}

We compared the model's prediction of spinal loading with the previous in vivo studies [3-8]. For this, the same tasks investigated in these studies were simulated, and the estimated intradiscal pressures were compared. We only included experimental data measured from healthy discs. The intradiscal pressures (in MPa) were calculated according to Eq. (4.1):

$$
I D P_{\text {model }}=\frac{F_{\mathrm{C}}}{D A \times C F}
$$

where $F_{\mathrm{C}}$ represents the predicted compressive force (in $\mathrm{N}$ ) at the intervertebral joint (Fig. 4.1), $D A$ represents the disc cross-sectional area (in $\mathrm{mm}^{2}$ ), and $C F$ represents a correction factor needed when calculating compression forces from intradiscal pressures [12]. Dreischarf et al. (2013) reported this correction factor to be $0.66 \pm 0.11$. Therefore, we calculated the intradiscal pressures by using three values for the correction factor $\left(C F_{\min }\right.$ $=0.55, C F_{\text {mean }}=0.66$, and $C F_{\max }=0.77$ ). For comparison purposes, the median values were plotted, and the error bars placed over the model's estimates represent the range due using different correction factors $\left(C F_{\min }\right.$ and $\left.C F_{\max }\right)$ (Figs. 4.2 and 4.3 . The pressure magnitudes measured at the L3/L4 disc were corrected by subtracting $0.25 \mathrm{MPa}$ as suggested by Nachemson and Elfstrom et al. (1970) [1, 3]. Intervertebral disc cross-sectional areas were quantified from the medical images and are given in Table 4.3 . 
Table 4.3: Intervertebral disc cross-sectional areas quantified from the CT images.

\begin{tabular}{ll}
\hline Disc & Area $\left(\mathrm{mm}^{2}\right)$ \\
\hline T6/T7 & 667 \\
T7/T8 & 727 \\
T9/T10 & 852 \\
T10/T11 & 954 \\
L3/L4 & 1367 \\
L4/L5 & 1398 \\
\hline
\end{tabular}

\subsubsection{Simulated tasks}

We simulated several quasi-static tasks with the model. Each task started from the standing upright position and finished when the specified maneuver (such as trunk flexion) was achieved. We performed these simulations for trunk and neck regions separately (referred to as trunk and neck tasks). For neck tasks, we distributed the total motion over the intervertebral joints between the skull and T1 segments (hence no trunk motion). Similarly, for the trunk tasks, we only specified the motion between $\mathrm{T} 1$ and pelvis segments (no neck motion) and partitioned the motion accordingly. The partitioning of the motion across each intervertebral joint took place according to the spinal rhythm matrices derived from previous reports [121-[126]. Furthermore, the total trunk motion was distributed between the pelvis and thoracic and lumbar regions of the spine. The amount of pelvic rotation in flexion and extension was calculated by using the lumbo-pelvic ratios reported by Tafazzol et al. (2014) [127]. 20\%, 73\%, and 83\% thoracic motion (out of the total rotation in the lumbar and the thoracic intervertebral joints) was used for the trunk flexion, extension, lateral bending, and axial rotation tasks, respectively. For the trunk tasks, we simulated standing upright, flexion $\left(60^{\circ}\right)$, extension $\left(15^{\circ}\right)$, lateral bending $\left(30^{\circ}\right)$, and axial rotation $\left(30^{\circ}\right)$ maneuvers. For the neck tasks, standing upright, flexion $\left(45^{\circ}\right)$, extension $\left(15^{\circ}\right)$, lateral bending $\left(20^{\circ}\right)$, and axial rotation $\left(20^{\circ}\right)$ were simulated. All the simulations were batch-processed and post-processed in Python 3.6 environment using AnyPyTools library.

\subsection{Results}

\subsubsection{Comparison of spinal loading with in vivo measurements}

Calculated intradiscal pressures at the lumbar region (L4/L5 and L3/L4 discs in Fig. 4.2) were highly correlated to previous experimental measurements [3]-7] $(\mathrm{R}=0.91$ and $\mathrm{RMSE}$ = 0.16). However, the model slightly underpredicted the compressive loading at the L3/L4 disc for certain postures. When the two levels were analyzed separately, the following correspondences were found: $\mathrm{L} 4 / \mathrm{L} 5(\mathrm{R}=0.95$ and $\mathrm{RMSE}=0.11)$ and $\mathrm{L} 3 / \mathrm{L} 4(\mathrm{R}=0.95$ and RMSE $=0.21$ ). The thoracic disc pressures (T6-T8 and T9-T11 discs in Fig. 4.3) predicted by the model were generally within the range previously reported [8], though a good correlation 

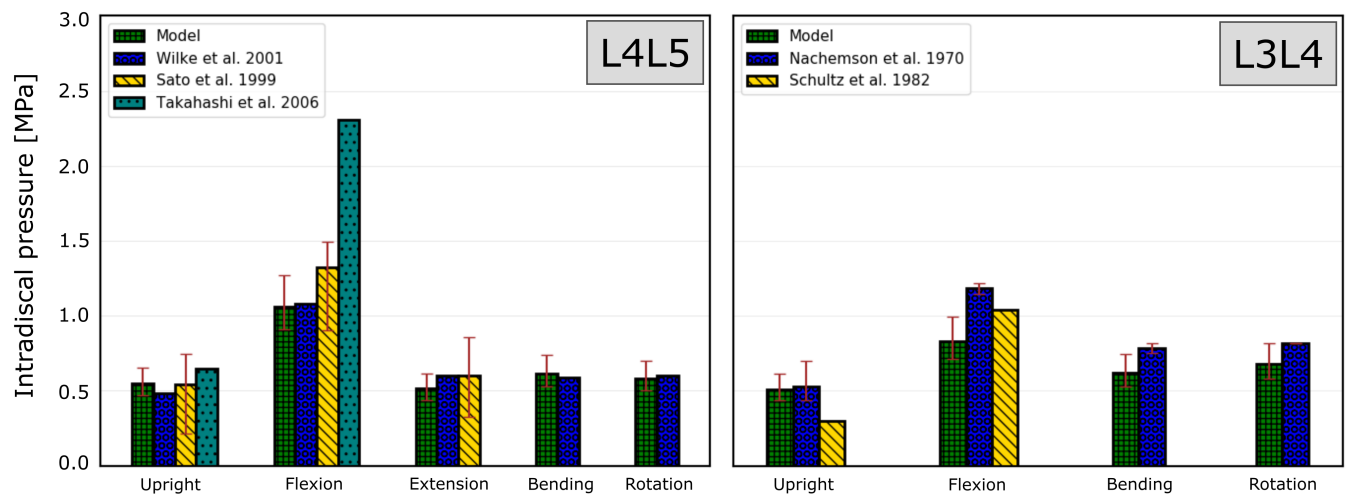

Figure 4.2: Intradiscal pressures at the L4/L5 and L3/L4 discs. The error bars placed over the model's predictions represent the range due using the minimum and maximum correction factors [12] whereas those placed over the experimental data show intersubject variance.

was lacking $(\mathrm{R}=0.45$ and $\mathrm{RMSE}=0.23$ ). The model predicted the trends (the pressure change compared to upright position) qualitatively well except for the lower thoracic discs in extension. Furthermore, the model captured the relative pressure levels between the middle and lower thoracic discs well except in lateral bending where our model predicted higher pressures at the middle thoracic discs than the lower levels. Moreover, the model predicted the trends well for the upright posture that the pressure levels (median values) from the highest to the lowest (similar to previous experiments) were as follows: T6-T8, T9-T11, L4/L5, and L3/L4. Further, we compared normalized resultant loads (as the percentage of the load at the upright standing) predicted by the model with the loads quantified from the instrumented devices implanted at the L1 vertebrae in Fig. 4.4 The model's estimates were within the measured range except in extension where the model overpredicted the normalized loads.

\subsubsection{Comparison of muscle function with previous studies}

Muscle forces and their activation patterns computed by the model were compared to previous modeling and experimental studies in Table 4.4. Although force magnitudes differed, in general, our model predicted similar activation trends to previous experimental and computational studies [4, 37, 128]. For example, erector spinae muscle group was prevalent in flexion and also played a role in lateral bending and axial rotation of the trunk. Latissimus dorsi was active during all the movements and was dominant in axial rotation. Similar to EMG measurements, except for extension, rectus abdominis was inactive. Obliquus externus abdominis and obliquus internus abdominis muscles followed similar patterns, and they were mainly active during trunk flexion, lateral bending, and axial rotation. 

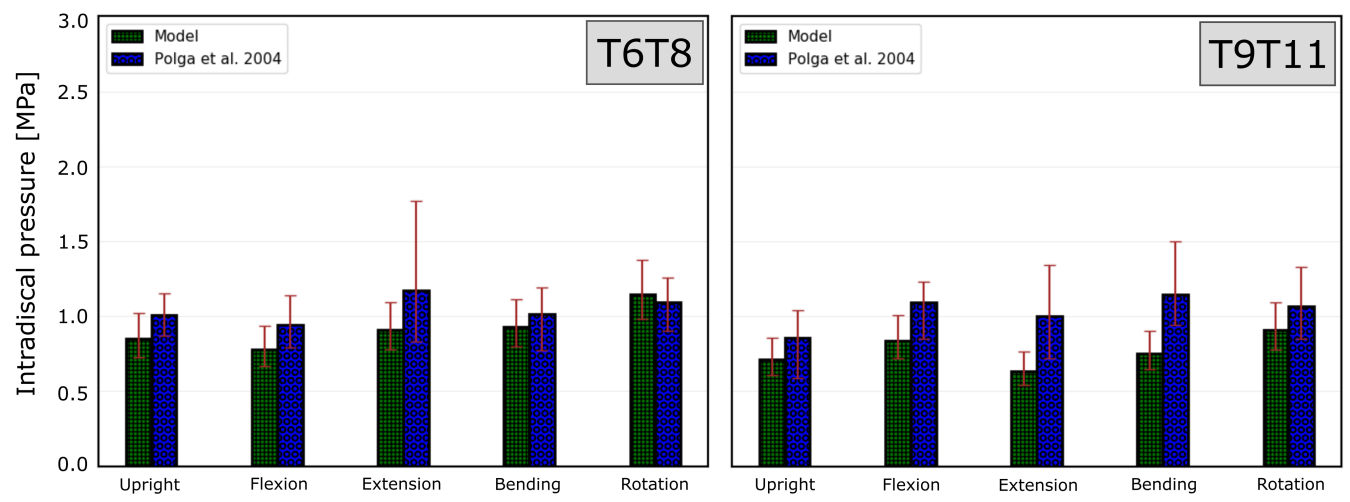

Figure 4.3: Intradiscal pressures at the middle (T6-T8) and lower T9-T11 thoracic discs. Our estimates were computed as the averages of the pressures calculated at the T6/T7 \& $\mathrm{T} 7 / \mathrm{T} 8$, and T9/T10 \& T10/T11 discs.

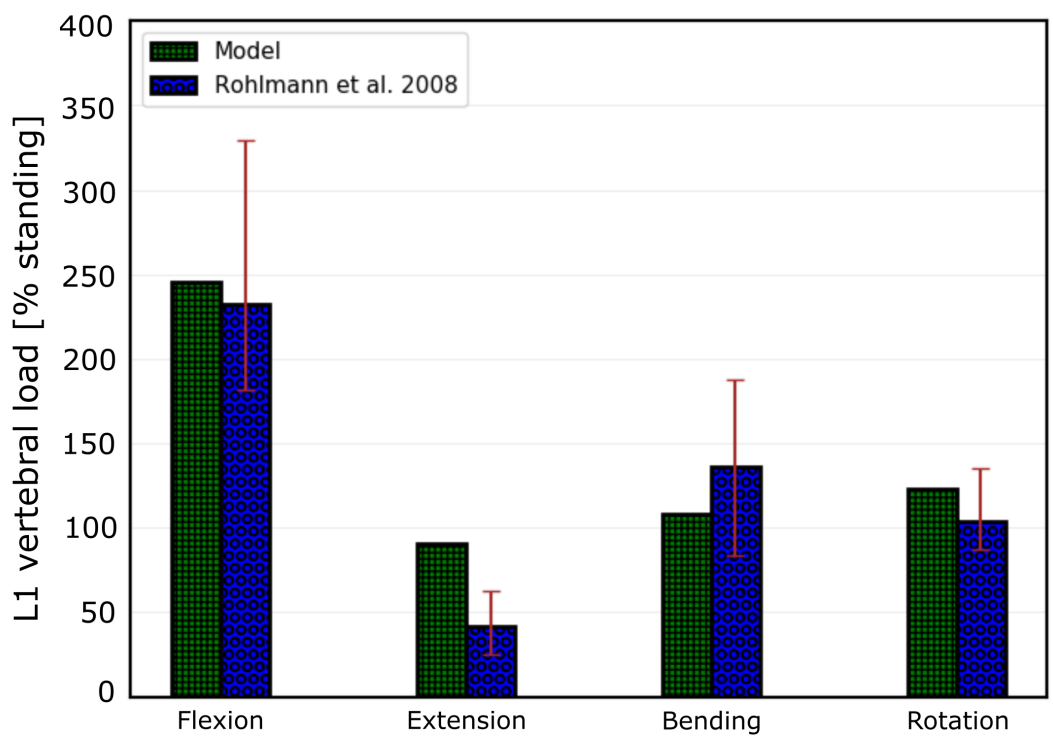

Figure 4.4: Resultant forces (normalized to standing) at the L1 vertebra. These forces were calculated as the average of the loads at the T12/L1 and L1/L2 disc joints. The error bars indicate the variance measured in two patients [10].

\subsubsection{Intervertebral disc joint forces}

\subsubsection{Compressive and shear forces at the standing upright position}

Compressive and anterior-posterior shear forces predicted at the intervertebral disc joints during standing were expressed as percentages of the total body weight (BW) and are shown in Fig. 4.5. The smallest and the largest compressive forces were found at the $\mathrm{C} 0 / \mathrm{C} 1$ (14\%) 
Table 4.4: Predicted forces $(\mathrm{N})$ in major trunk muscles compared to previous studies.

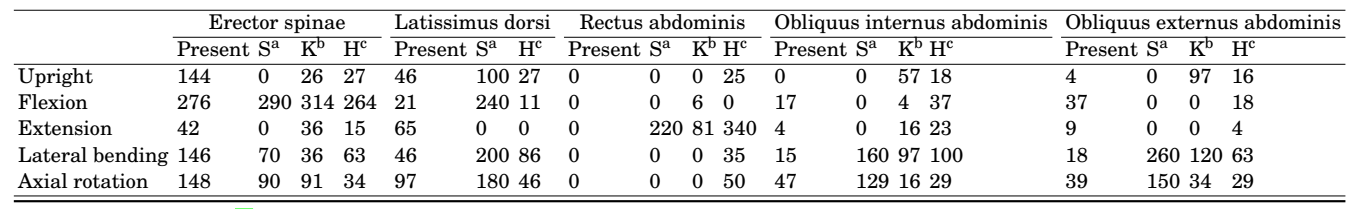

a Schultz et al. (1982) 4

b Khurelbaatar et al. (2015)

${ }^{\mathrm{c}}$ Han et al. (2012) 128

Table 4.5: Intervertebral disc inclinations on the sagittal plane.

\begin{tabular}{ll}
\hline Disc & Angle $\left(^{\circ}\right)$ \\
\hline C2/C3 & 0.0 \\
C3/C4 & -14.3 \\
C4/C5 & -23.6 \\
C5/C6 & -27.0 \\
C6/C7 & -30.3 \\
C7/T1 & -36.0 \\
T1/T2 & -37.3 \\
T2/T3 & -37.2 \\
T3/T4 & -34.9 \\
T4/T5 & -31.2 \\
T5/T6 & -22.0 \\
T6/T7 & -10.9 \\
T7/T8 & -6.4 \\
T8/T9 & -0.2 \\
T9/T10 & 3.6 \\
T10/T11 & 5.3 \\
T11/T12 & 9.4 \\
T12/L1 & 11.6 \\
L1/L2 & 9.0 \\
L2/L3 & 1.6 \\
L3/L4 & -12.2 \\
L4/L5 & -35.6 \\
L5/S1 & -44.3 \\
\hline
\end{tabular}

and L5/S1 (101\%) joints, respectively. The compressive forces increased from cervical to lumbar, and there were some fluctuations at the thoracolumbar junction and middle lumbar levels. The median values for the cervical (C0-T1), thoracic (T1-L1), and lumbar (L1-S1) regions were $20 \%, 70 \%$, and $95 \%$ of body weight, respectively.

Shear forces were remarkably lower than the compressive forces throughout the spine, and they were relatively higher at the upper thoracic and lower lumbar levels. The lowest and the highest shear forces were found at the $\mathrm{C} 3 / \mathrm{C} 4(0.2 \%)$ and L5/S1 (61\%) joints, respectively. Regional medians were as follows: cervical (4\%), thoracic (7\%), and lumbar (28\%). The correlation analysis between the disc inclinations (Table 4.5) and shear forces did not reveal a significant relation $(\mathrm{R}=0.64)$ across all discs. However, when the three regions were analyzed separately, we found good to strong relations between disc inclination and shear force: cervical $(R=0.69)$, thoracic $(R=0.95)$, and lumbar $(R=0.99)$. 


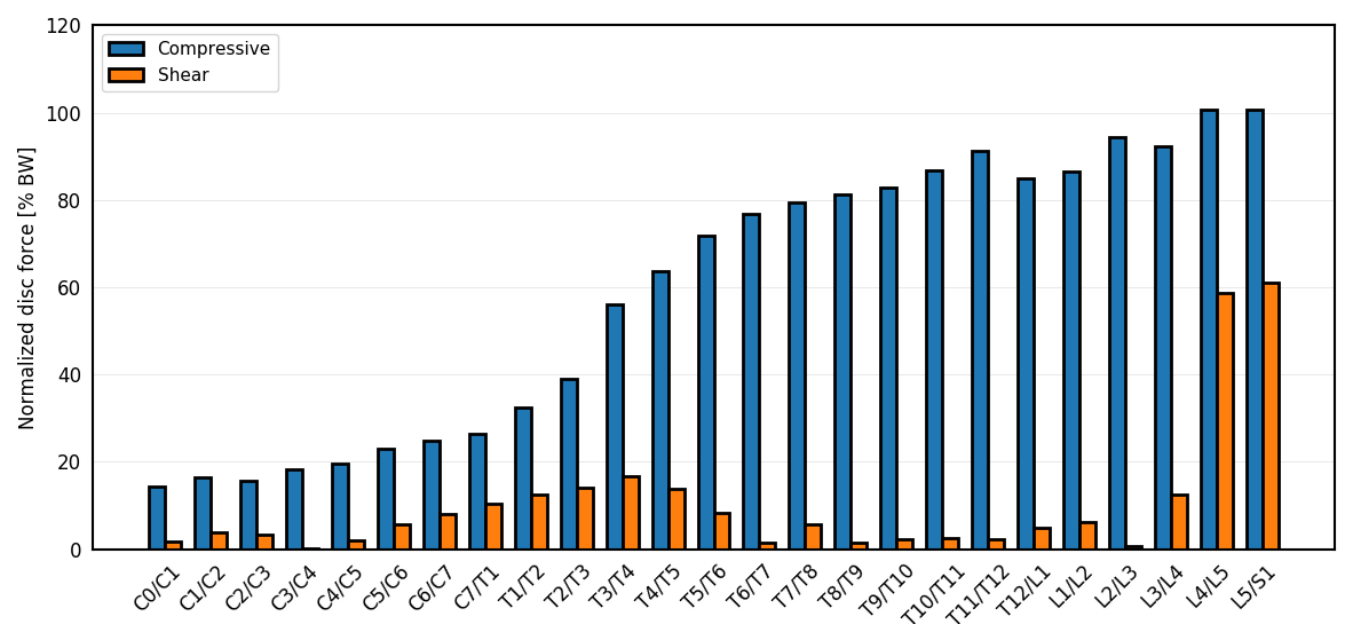

Figure 4.5: Compressive and anterior-posterior shear forces at the intervertebral disc joints during standing upright. The forces were expressed as percentages of the total body weight (BW) of the cadaver.

\subsubsection{Compressive forces during trunk tasks}

We present the model's compressive forces at the thoracic and lumbar discs during the motion for the trunk (Figs. 4.6 and 4.7): standing upright, flexion, extension, lateral bending, and axial rotation. The forces were computed as percentages of the total body weight of the cadaver.

In general, flexion caused the largest spinal loads at the lumbar and thoracic discs at the final positions among all the maneuvers. When the same trunk rotations were considered $\left(30^{\circ}\right.$ flexion, lateral bending, and axial rotation), axial rotation generally induced higher loading at the thoracic discs, whereas forward flexion induced higher loads in the lumbar discs. The disc forces mainly increased with increasing trunk motion except in extension where they decreased at the lower thoracic and lumbar discs. Furthermore, in general, the forces increased caudally.

In forward flexion, the compressive forces at the upper and lower thoracic discs as well as the lumbar discs gradually increased whereas they slightly decreased at the middle thoracic discs with increased trunk inclination. At $60^{\circ}$ trunk flexion, the highest and lowest forces were noted for the L5/S1 (250\%) and T3/T4 (49\%) joints, respectively.

In extension, the forces in the thoracolumbar and lumbar regions slightly decreased until around $10^{\circ}$, after which they gradually increased. The forces at the upper and middle thoracic joints slightly increased, and the lower thoracic discs had smaller compression 

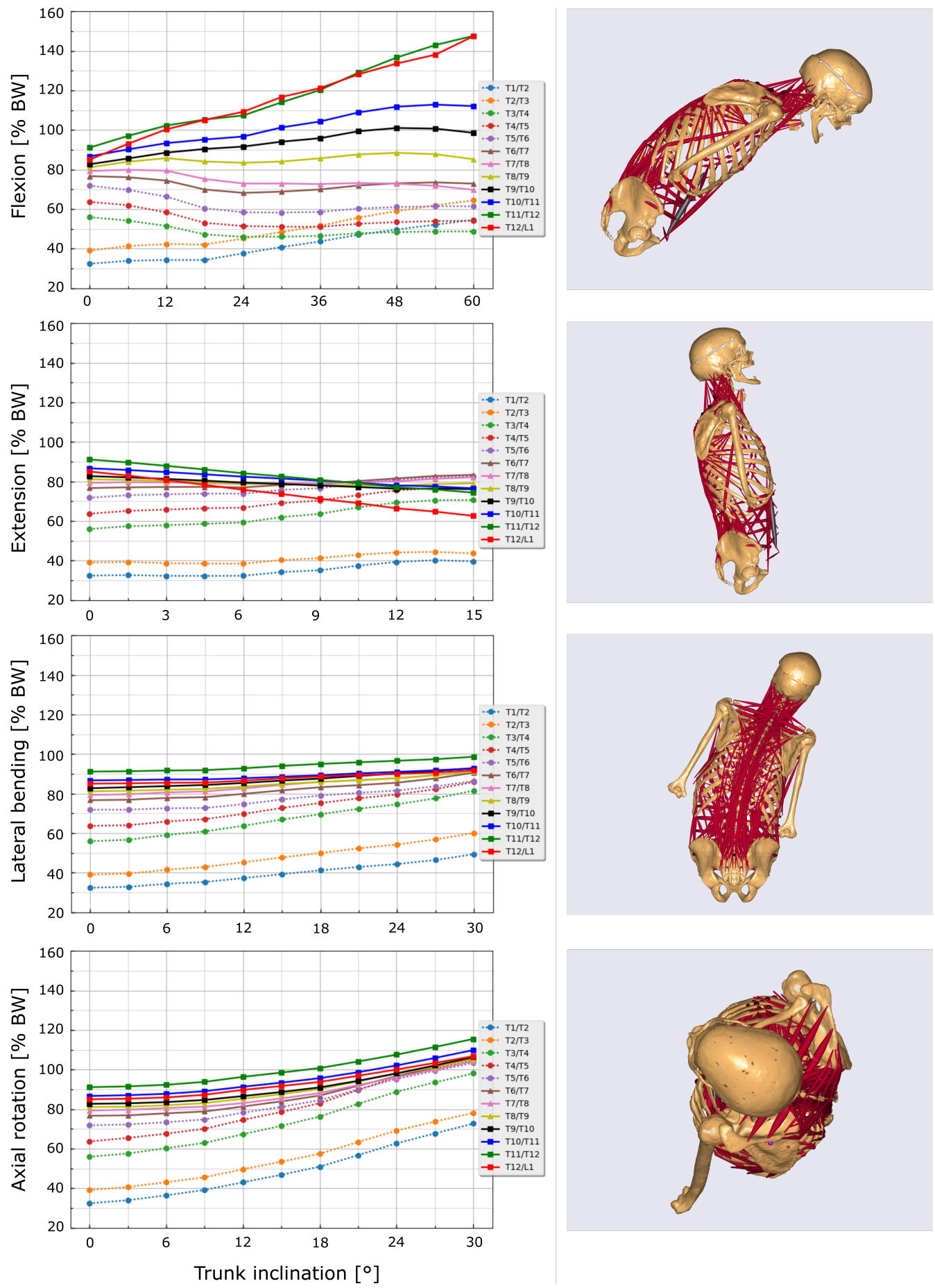

Figure 4.6: Disc compressive forces in the thoracic spine as functions of trunk inclination. The end postures in each task are shown next to the plots. 

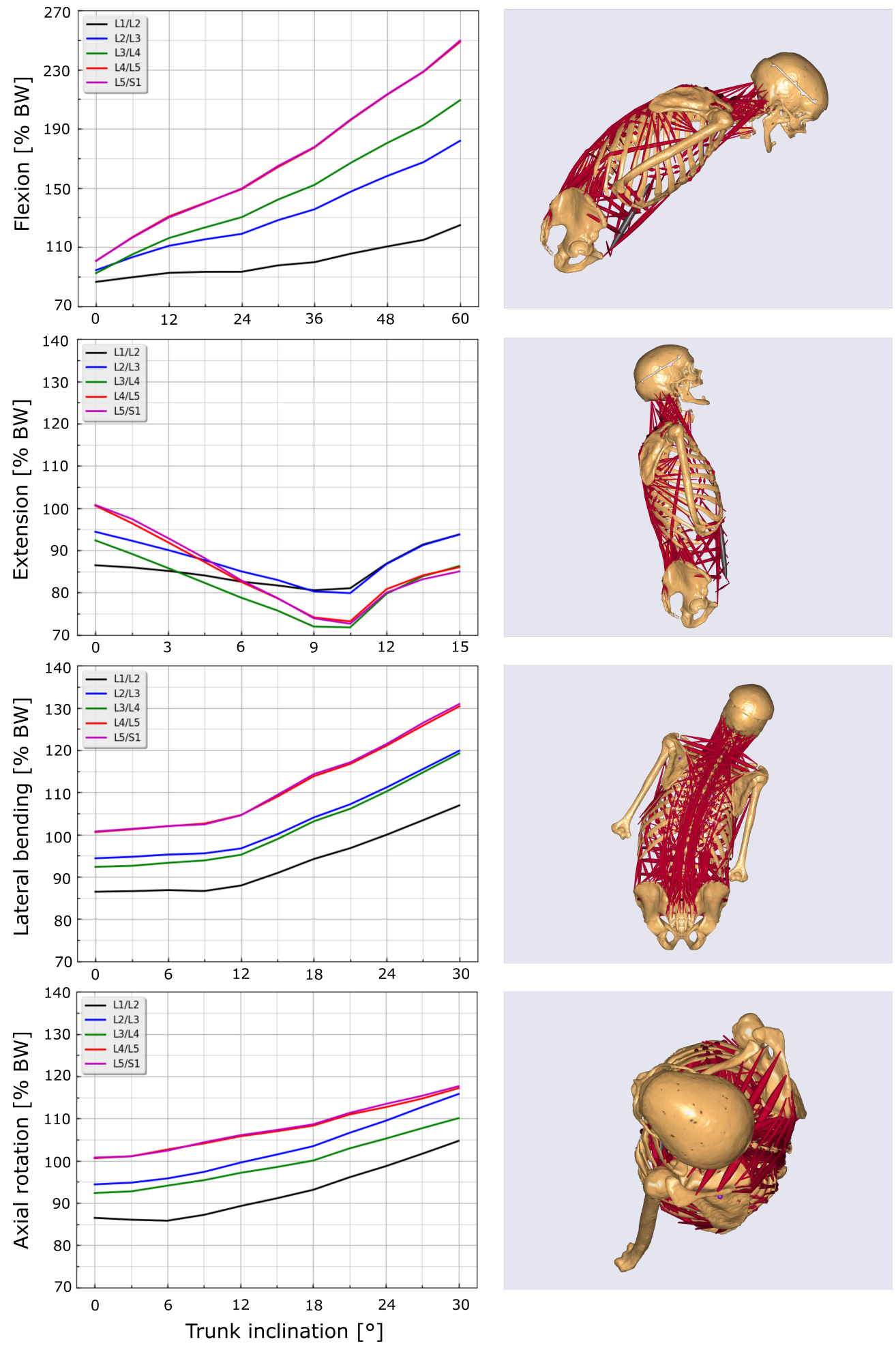

Figure 4.7: Disc compressive forces in the lumbar spine as functions of trunk inclination. The end postures in each task are shown next to the plots. 
(10-23\% decrease) compared to upright posture. At the end of the maneuver, the L2/L3 disc had the highest compression (94\%), and the T1/T2 disc had the lowest (40\%).

Lateral bending increased the compression at all the discs gradually and almost linearly, around 6 to $25 \%$ rise at the thoracic discs and 20 to $30 \%$ rise at the lumbar discs. The highest and lowest forces were predicted for the L5/S1 (131\%) and T1/T2 (49\%) joints, respectively.

Similar to lateral bending, axial rotation increased the disc compressions almost linearly at all the joints. Compared to lateral bending, axial rotation led to relatively higher increases in the thoracic region yet smaller in the lumbar region. We found the largest and smallest forces as $118 \%$ at the L5/S1 joint and $73 \%$ at the $\mathrm{T} 1 / \mathrm{T} 2$ joint, respectively.

\subsubsection{Compressive forces during neck tasks}

The compressive forces at the cervical discs (Fig. 4.8) were much lower than those of the lumbar and thoracic discs. In general, the forces increased in flexion, lateral bending, and axial rotation but decreased in extension. Lateral bending induced the largest forces at the lower discs (43\% at $\mathrm{C} 7 / \mathrm{T} 1)$ whereas axial rotation did at the upper discs (56\% at $\mathrm{C} 1 / \mathrm{C} 2)$.

In flexion, the highest and lowest forces were noted at the C7/T1 (37\%) and C2/C3 (21\%) joints, respectively. Compared to the neutral position, the force increases ranged from $3 \%$ (C6/C7) to $17 \%(\mathrm{C} 0 / \mathrm{C} 1)$.

In extension, the compressions gradually decreased except at the $\mathrm{C5} / \mathrm{C} 6$ and $\mathrm{C} 6 / \mathrm{C} 7 \mathrm{discs}$ where they almost did not change compared to neutral position. The model predicted the highest and lowest forces at the $\mathrm{C} 6 / \mathrm{C} 7(25 \%)$ and $\mathrm{C} 0 / \mathrm{C} 1(9 \%)$ joints, respectively.

During early lateral bending, the forces did not change much until around $4^{\circ}$ but consistently increased afterward at all the joints roughly linearly. The highest and lowest forces were found at the $\mathrm{C} 7 / \mathrm{T} 1$ (43\%) and C4/C5 (32\%) discs.

In axial rotation, the forces first did not change much until around $10-14^{\circ}$ but increased rapidly afterward at all the joints. At the final position, the upper joints were loaded the largest. All the joints had significantly higher forces at the end of the maneuver when compared to upright position. The $\mathrm{C} 1 / \mathrm{C} 2$ joint had the largest force (56\%) and $\mathrm{C} 4 / \mathrm{C} 5$ (35\%) the smallest. 

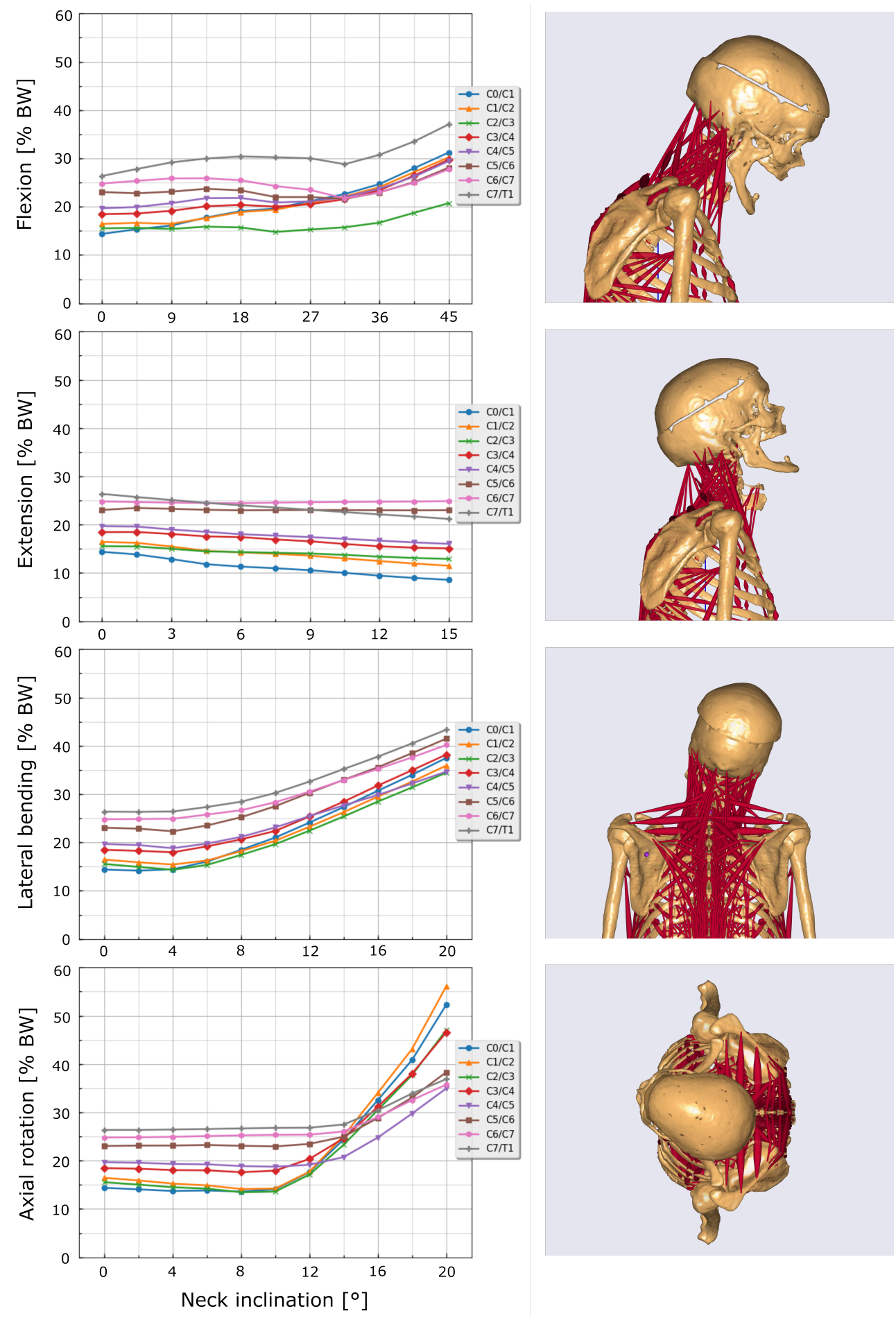

Figure 4.8: Disc compressive forces in the cervical spine as functions of neck inclination. The end postures in each task are shown next to the plots. 


\subsection{Discussion and conclusion}

Accurate characterization of spinal loads is fundamental to understanding normal and pathological spine functioning [129], injury prevention during occupational tasks [28], optimization of surgical interventions, and pre-clinical testing of implants. In the past, spinal loads have been measured through intervertebral disc pressures and telemetered vertebral implants [3]-8, 10]. Despite these previous experimental studies, our understanding of the relation between the movement and the spinal loads remains largely unexplored mainly due to technical difficulties in experimentation and the related ethical concerns. Musculoskeletal modeling accommodates great opportunities to study spine biomechanics in depth and to estimate in vivo loads.

In this study, we developed a complete and coherent musculoskeletal model of the entire human spine by incorporating the anatomical dataset reported in earlier work [84, 105]. When developing this model, skeletal and muscle morphologies were reconstructed from this dataset while segment mass properties, joint definitions, and stiffness properties, and spinal rhythms were implemented based on previous investigations. Since all the muscle and skeletal data were obtained from one single spine, our model essentially represents a subjectspecific musculoskeletal system and, unlike generic models, it does not require combining anatomical data from other datasets [38]. This model includes body segments, mechanical joints to mimic their articulations, and muscle groups and can predict spinal loading using an inverse dynamic approach. The inherent redundancy in the musculoskeletal system is solved using an optimization criterion in which muscle activations are computed based on minimization of muscle fatigue. Our model has several particular features, such as the incorporation of individual vertebrae (cervical, thoracic, and lumbar), flexible ribcage, and comprehensive muscular anatomy which included various deeper muscles in the spine. Incorporation of non-rigid cervical and thoracic spines enables more realistic and detailed investigations of the muscle activation patterns and the spinal loads [40]. These features might be particularly important when studying the joints connecting different regions of the spinal column (for example, $\mathrm{C} 7 / \mathrm{T} 1$ joint) as the changes in moment arms of the muscles spanning these joints would be better implemented.

We calculated the intradiscal pressures from predicted compressive forces at the disc joints during physiological trunk movements. For this, we simulated several trunk postures similar to previous experiments where in vivo pressures at certain lumbar and thoracic discs were measured. Disc pressures estimated by our model at the L4/L5 and L3/L4 levels were overall in good agreement $(\mathrm{R}=0.91)$ with the previous data from several investigations [37]. For the third lumbar disc, we used the data measured in two different studies [3, 4] These studies reported intradiscal pressures in a broad range $(0.3-0.7 \mathrm{MPa})$ and found increases in pressures during trunk flexion. Our model underpredicted the compression 
at this disc level during flexion and side lateral bending but predicted the increase trends compared to upright position well (Fig. 4.2). Polga et al. (2004) reported the pressures at the middle and lower thoracic discs from six healthy individuals [8]. Polga's data also showed significant variations for all the trunk postures yet relatively higher variations in extension and lateral bending. Nevertheless, the model's estimations were generally within the range reported, and it predicted the change in pressures compared to upright position qualitatively well except for the lower discs during extension (Fig. 4.3). In addition, the model captured the relative pressures between the middle and lower discs (except in lateral bending) in accordance with the measurements. These variances between the in vivo data, as well as the discrepancies in our findings, can perhaps be explained by subject-specific factors such as spinal curvature and rhythm or simplified modeling of the arms as lumped masses [86]. Finally, our estimations at the thoracic and lumbar levels during an upright position were in excellent agreement with the experimental data. We found that the pressures decreased caudally from $0.85 \mathrm{MPa}$ at T6-T8 and $0.71 \mathrm{MPa}$ at T9-T11 to $0.51 \mathrm{MPa}$ at L3/L4 but then increased to $0.55 \mathrm{MPa}$ at L4/L5 disc [1]. In this study, we generally achieved good agreements with the previous in vivo measurements at the lumbar and thoracic discs. This demonstrated a preliminary validation of our model and gave us the confidence to study the development of the compression forces at the intervertebral discs during basic activities of daily living. It is important to note that further validation of the model in terms of muscle forces and joint moments under more demanding activities is necessary. This will enable to expand the model's credibility for investigating occupational tasks such as manual materials handling and will be the subject of future research.

During standing, predicted compressive forces (\% BW) at all levels (Fig. 4.5) were consistently higher than those reported in previous computational models [28, 34, 37, 40, 130] but similar to measured data. For example, previous models' estimations at the L4/L5 disc ranged from $52 \%$ to $89 \%$ BW whereas in vivo data (derived from Dreischarf's review paper [1]) indicated huge variations: Wilke et al. (2001) [6] (71\% - 99\%), Sato et al. (1999) [5] (22\% - 143\%), and Takahashi et al. (2006) [7] (54\% - 75\%). We found compression of $101 \%$ BW at the L4/L5 disc during standing. For the T9/T10 disc, previous models found ratios ranging from $31 \%$ to $77 \%$ while data from Polga et al. (2004) [8] suggests between $\sim 33 \%$ and $\sim 81 \%$ (estimated from the reported range and disc areas [131]). We found a comparable compressive force of $83 \% \mathrm{BW}$ at the T9/T10 level. We saw that similar variations existed for the third lumbar and middle thoracic discs and that our estimations fit within the in vivo data reported. Furthermore, our predictions of compression forces were closest to those reported from a generic musculoskeletal model of the thoracolumbar spine which assumed a single-lumped rigid body representation of the neck and overpredicted intradiscal pressures at the thoracic discs [34]. Predicted anterior-posterior shear forces during standing (Fig. 4.5] were also generally higher than those found in the aforementioned models which reported varying shear force distributions at the lumbar spine. Previous models estimated the forces 
between $\sim 4 \%$ and $\sim 10 \% \mathrm{BW}$ at the first lumbar disc compared to the current study (6\%) and the experimental measurements $\sim 10 \%$ [10]. This indicated that our prediction of the anterior-posterior shear at the L1/L2 level was in accordance with the previous models and the in vivo data. Unfortunately, we are unaware of any experimental data to validate our predictions further at the other levels. The variations between the previous models and our cadaver-based model are possibly due to the differences in the simulated muscle and skeletal anatomy or modeling practices.

Dreischarf et al. (2016) [12] elaborated that the compressive forces at the healthy intervertebral discs can be estimated from the intradiscal pressures during all the maneuvers studied in this work except extension. They found increasing facet joint forces, decreasing disc forces and discussed the non-proportionality of the pressure and compression force during extension because the pressure increased in vivo []. In extension, we found decreasing compression forces at the thoracolumbar discs and slightly increasing forces at the middle thoracic discs with increased trunk inclinations (Fig. 4.6. At the lumbar discs, the forces descended during early extension but increased later for trunk angles larger than $\sim 10^{\circ}$ (Fig. 4.7). This increase was caused by saturated activities of deep muscles such as spinalis thoracis, rotatores, and multifidus to balance the external loads around the thoracolumbar joints. Due to this force increase at the L1/L2 joint (although a decrease at the T12/L1 joint), our model, similar to the previous models [33, 37, 128], overpredicted the normalized loads at the L1 level in extension (Fig. 4.4). Although the rises at the middle thoracic discs and the drops at the lumbar discs during early extension were in accordance with the in vivo data, the decreases at the lower thoracic discs and the increases in the lumbar region were in disagreement [8, 10]. These suggest that our predictions for extension might not be very reliable after all and necessitates a further study. In line with the experimental studies [6, 8, 10], we found increased compressions at all the trunk discs during lateral bending and axial rotation maneuvers. Similarly, our simulations indicated increased compressive forces at the lower thoracic discs and lumbar regions and slightly decreased forces at the middle thoracic discs during trunk flexion. These findings were also in agreement with the in vivo measurements and differed from a previous thoracolumbar spine model which reported higher compression forces and thus intradiscal pressures (compared to upright position) at the middle thoracic discs [34].

The predicted compressive forces at the cervical discs were significantly lower than those of the thoracic and lumbar discs (Fig. 4.8). Due to the lack of in vivo data on cervical disc pressures, it was not possible to validate our findings for the cervical levels. But when comparing our model's findings to those of previous models, they give similar results. Snijders et al. (1991) [132] found in their cervical biomechanical model that disc forces increased during flexion, reached their minimums in extension, increased in lateral bending, and did not change much until $35^{\circ}$ axial rotation but increased fast with further axial rotation. 
Our findings for the cervical joints were in close agreement with these observations. We found that the disc forces increased significantly after $\sim 14^{\circ}$ in axial rotation instead of $35^{\circ}$. Furthermore, the normalized compressive forces at the neutral position were similar to a previous cervico-thoraco-lumbar spine model [37].

Several limitations affect the data presented in this work. Firstly, our model used a simplified approach for modeling the shoulder-arm complex. This practice facilitated including trunk muscles such as latissimus dorsi and trapezius that played roles during lateral bending and axial rotation. We estimated and applied external forces and moments at the third thoracic vertebrae to model the loads due to these extremities [109]. Thus, our model is not usable for tasks that cause large arm forces. Secondly, we used a simple muscle model where force-length and force-velocity relationships were not considered. For simplicity, we opt to not include them as some muscle parameters such as optimal fiber length and tendon slack length would require further calibrations. Thirdly, the intervertebral disc articulations were simplified as spherical joints which lacked translational DoFs. Recent studies discussed that neglecting translational DoFs and thus assuming fixed locations for joint centers of axial rotation might affect the joint reaction and muscle forces [133, 134]. Fourthly, we did not model the facet joints. Earlier studies discussed that the facet joints will cause a decrease in the transmission of forces through the anterior column during extension [12, 33]. Fifthly, we did not include spinal ligaments but included their collective rotational stiffnesses linearly in the intervertebral and ribcage joints. The lack of ligaments or simplifying their non-linear mechanical behaviors in the model would change the load sharing patterns between the muscles and thus affect our findings [135]. Sixthly, our cadaver-based model was not scaled to match anthropometric features of the subjects volunteered in the previous in vivo measurements of spinal loads. We found it more realistic to compare disc pressures as was done in this study rather than applying complex scaling protocols which were not available. Seventhly, this subject-specific model represents an elderly male with a body height of $154 \mathrm{~cm}$ and a mass of $51 \mathrm{~kg}$. Thus, the applicability of the findings presented in this work might be limited when individuals with different anthropometric features and morphologies are studied. Finally, we refer the reader to our previous works where limitations peculiar to the anatomical dataset used in this model were described in detail [84, 105].

\section{Conflict of interest}

Pavel E. Galibarov works in AnyBody Technology A/S. None of the other authors have any financial or personal relationships with other people or organization that could inappropriately influence their work. 


\section{Funding}

We thankfully acknowledge the financial support by fonds NutsOhra (1302-032).

\section{Acknowledgments}

We thank Dominika Ignasiak and Stephen J. Ferguson from Institute for Biomechanics, Switzerland for giving us the opportunity to study their thoracolumbar spine model.

Ethical Approval: Not required. 


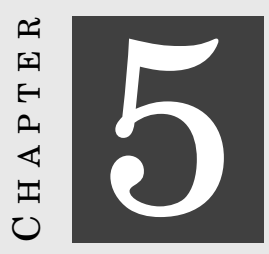

\title{
SENSITIVITY OF MUSCLE AND INTERVERTEBRAL DISC FORCE COMPUTATIONS TO VARIATIONS IN MUSCLE ATTACHMENT SITES
}

\begin{abstract}
The current paper aims at assessing the sensitivity of muscle and intervertebral disc force computations against potential errors in modeling muscle attachment sites. We perturbed each attachment location in a complete and coherent musculoskeletal model of the human spine and quantified the changes in muscle and disc forces during standing upright, flexion, lateral bending, and axial rotation of the trunk. Although the majority of the muscles caused minor changes (less than 5\%) in the disc forces, certain muscle groups, for example, quadratus lumborum, altered the shear and compressive forces as high as $353 \%$ and $17 \%$, respectively. Furthermore, percent changes were higher in the shear forces than in the compressive forces. Our analyses identified certain muscles in the ribcage (intercostales interni and intercostales externi) and lumbar spine (quadratus lumborum and longissimus thoracis) as being more influential for computing muscle and disc forces. Furthermore, the disc forces at the L4/L5 joint were the most sensitive against muscle attachment sites, followed by T6/T7 and T12/L1 joints. Presented findings suggest that modeling muscle attachment sites based on solely anatomical illustrations might lead to erroneous evaluation of internal forces and promote using anatomical datasets where these locations were accurately measured. When developing a personalized model of the spine, certain care should also be paid especially for the muscles indicated in this work.
\end{abstract}

This chapter has been submitted for publication as: Riza Bayoglu, Ogulcan Guldeniz, Nico Verdonschot, Bart Koopman, Jasper Homminga, "Sensitivity of muscle and intervertebral disc force computations to variations in muscle attachment sites". 


\subsection{Introduction}

Computational models of the musculoskeletal system provide great opportunities to improve existing diagnosis and treatment practices for musculoskeletal and neuromuscular disorders. In the spine, detailed assessments of internal loads in the spinal tissues and muscle coordination strategies during trunk movement can help to achieve these clinical objectives [2]. For example, Bruno et al. (2017) elaborated on the higher rates of vertebral fractures in the middle thoracic and thoracolumbar regions by computing vertebral compressive forces and the associated risk factors for a series of daily activities [136]. The credibility of such biomechanical analyses depends largely on how well the models represent the true skeletal and muscular anatomy in all its complexity. Since muscle lines-of-action determine torque contributions at the joints, modeling muscle attachment sites at the bones-that is muscle origin, insertion, and via points-requires special attention for accurate assessment of joint and muscle forces.

In the past years, attachments of major spinal muscles were reported mostly through anatomical drawings that were created in detailed dissection studies on the lumbar spine [42, 53, [58, 64] and cervical spine [38, 52]. These illustrations were prepared by visually inspecting the tendinous attachments of muscle bundles at the bones and estimating average locations across the cadavers studied rather than measuring the positions. Subsequently, the majority of generic musculoskeletal models adopted these illustrations for defining muscle lines-of-action [26-28, 34]. Other studies introduced novel techniques to adjust muscle paths and cross-sectional areas of major trunk muscles based on 2D medical images [31, 33, 41]. Recently, we completed an entire anatomical dataset measured from one single human spine [84, 105]. In this dataset, the coordinates of muscle attachment sites (quantified by optical tracking technology), spinal geometry, and comprehensive morphological parameters of 321 muscle-tendon elements were reported. Subsequently, a complete and coherent musculoskeletal model for the entire human spine (the Twente Spine Model) was developed based on this dataset [137]. With musculoskeletal models in general, and thus with ours, it is an important question that how sensitive the outcomes are to errors in the input data.

Previously, several authors investigated the sensitivity of their models against the locations of intervertebral center of rotation [138], spine rhythms and abdominal pressure [85], ligament stiffness [139], and vertebral morphology [48]. Muscle recruitment during trunk exertions is dependent on muscle lines-of-action, however, there is only one study which aimed to investigate the sensitivity of muscle and intervertebral disc force computations against muscle attachment sites in the spine. Nussbaum et al. (1995) varied lines-of-action of some lumbar muscles within anatomically feasible ranges to reflect inter-individual differences and studied the effects on predicted muscle and disc forces [140]. Unfortunately, 
their model included only a few muscle groups, and the findings were limited to a single disc level in the lumbar spine. Thus, the aim of the present study is to assess the effects of variations in muscle attachment sites on the computed muscle and intervertebral disc forces in a musculoskeletal model of the spine. We focused on potential measurement errors in muscle origin, insertion, and via point locations and subsequent implementation in musculoskeletal models. Specifically, each attachment location was systematically perturbed, and the global reaction of the muscular system to the corresponding perturbation was calculated. We also analyzed the changes in compressive and shear forces at the T6/T7, $\mathrm{T} 12 / \mathrm{L} 1$, and $\mathrm{L} 4 / 5$ discs.

\subsection{Materials and methods}

The Twente Spine Model was used to assess the sensitivity of muscle and intervertebral disc joint reaction force computations to muscle attachment locations [137]. This model was based on a complete and coherent anatomical dataset measured from one male cadaver (height: $154 \mathrm{~cm}$, mass: $51 \mathrm{~kg}$ ) [84, 105] and was made right to left symmetrical (see Fig. 5.1). It included 60 bony segments (cervical, thoracic, and lumbar vertebrae, ribs, skull, sternum, hyoid, thyrohyoid, clavicles, scapulas, humeri, sacrum, and pelvis) and 351 muscle-tendon force actuators (per body side, see Table 5.1) and had 193 degrees-of-freedom (DoF). Intervertebral articulations were modeled as spherical, costo-vertebral and costotransverse articulations as compound revolute, and the costo-sternal articulations as six DoF joints. Further, the lines-of-action of each muscle-tendon actuator (muscle element) was constructed by its origin and insertion where the force is transferred along its path. We used additional via points for muscle elements with a curved lines-of-action to represent their path better. At via points, muscle force is transferred in the direction of a line that bisects the angle formed by the muscle on either side of the point. The isometric strength of each muscle element was calculated by multiplying its physiological-cross sectional area (PCSA) by the specific muscle tension $\left(100 \mathrm{~N} / \mathrm{cm}^{2}\right.$, [141]. For simplicity, muscle force-length and force-velocity relationships were not considered. The model relied on inverse dynamics and employs a static optimization criterion (cubic polynomial) for computing muscle forces [142].

The Twente Spine Model's estimations of intradiscal pressures have previously been validated against in vivo disc pressures and loads from instrumented vertebral implants both quantitatively and qualitatively [137]. Briefly, the same tasks-consisting of the flexion, extension, lateral bending, and axial rotation of the trunk-were simulated, and intradiscal pressures were calculated from predicted disc compressive forces. Good correlations between calculated and measured intradiscal pressures were found for the lumbar (L3/L4 and L4/L5) and thoracic (T6 - T8 and T9 - T11) discs. Furthermore, normalized resultant loads (as the percentage of the load at the upright position) at the L1 vertebra were also in good agreement with the experimental data [10]. However, the model overpredicted the 

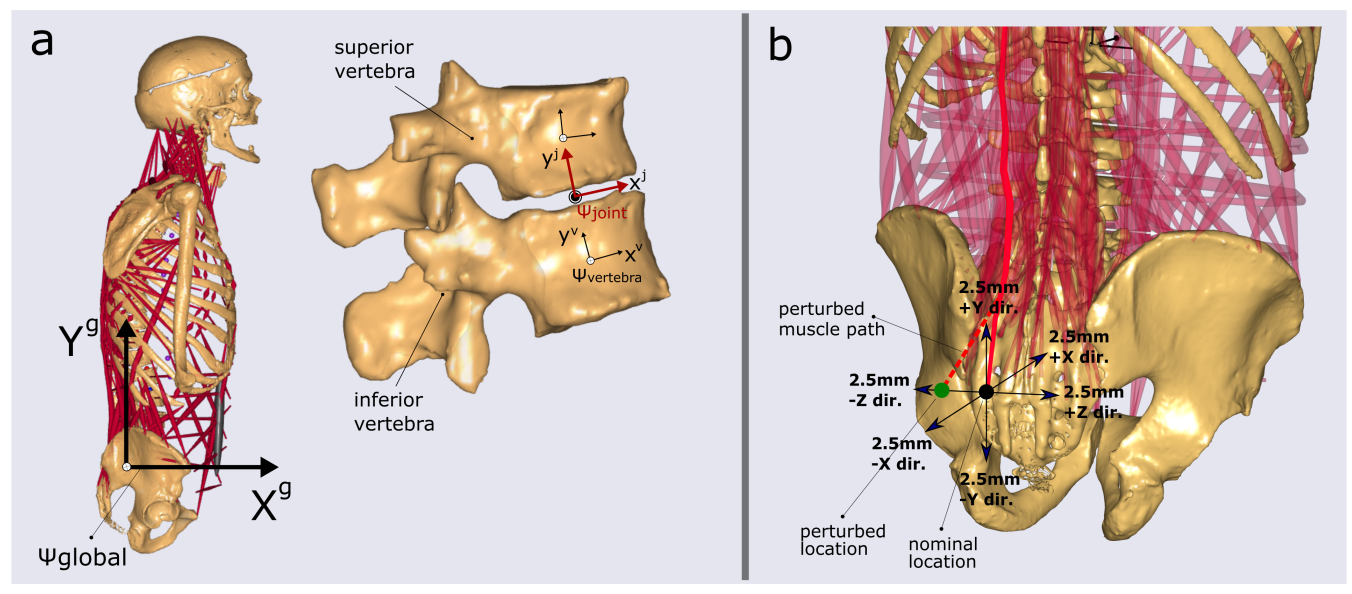

Figure 5.1: (a) The musculoskeletal model used in this study, bones are displayed in beige and muscle-tendon elements in red. The reference frame depicted in the sagittal view is the global reference frame $\left(\psi_{\text {global }}\right)$. In the global frame, $+X^{g},+Y^{g}$, and $+Z^{g}$-axes point anteriorly, cranially, and laterally (to the right side of the cadaver), respectively. The vertebral ( $\left.\psi_{\text {vertebra }}\right)$ and intervertebral disc joint reference frames $\left(\psi_{\text {joint }}\right)$ are shown for one motion unit. The orientations of the joint reference frames were defined as the average of their corresponding superior and inferior vertebrae reference frames [143]. The compressive force at the intervertebral disc is calculated along the $y^{j}$-axis of the joint reference frame while the shear is the force component on the $x z$ plane. (b) Here the perturbations of the muscle attachment locations are illustrated. In this example, the measured location (nominal) was perturbed $2.5 \mathrm{~mm}$ along the negative $Z^{g}$-axis. The modified muscle path due to this perturbation is indicated by the red dashed line.

normalized loads during trunk extension.

Several quasi-static trunk movements were simulated with the model: standing upright, flexion, lateral bending, and axial rotation (see Table 5.2). Each movement started from the upright posture, lasted ten seconds, and finished when the prescribed movement was achieved. We only specified the motion between the first thoracic vertebra and pelvis segments (hence, no motion at the cervical joints) and distributed the total motion across the thoracolumbar joints in accordance with previous reports [121-[127].

In this study, we only investigated the trunk muscles since our focus was to assess the sensitivity of internal force predictions within this region. In total, 22032 (918 attachment locations-total number of origins, insertions, and via points-per side $\mathrm{x} 6$ directions $\mathrm{x} 4$ movements) perturbations were simulated. In every simulation, a single attachment point was perturbed from its nominal location along one direction, and all the other attachments were left intact (Fig. 5.1p). However, the attachment points shared by different muscle elements were perturbed simultaneously. Since this model was built right to left symmetrical on the 
Table 5.1: Muscles included in the Twente Spine Model [137]. In this study, only the muscles located in the thoracic spine, ribcage, and lumbar spine were perturbed. Abbreviations used for the muscle names are indicated inside the parenthesizes.

\begin{tabular}{ll}
\hline Region & Muscle \\
\hline Cervical spine & Iliocostalis cervicis, Levator scapulae, Longissimus capitis, Longissimus cervicis \\
& Longus capitis, Obliquus capitis inferior, Obliquus capitis superior, Omohyoid \\
Rectus capitis anterior, Rectus capitis lateralis, Rectus capitis posterior major \\
Rectus capitis posterior minor, Scalenus anterior, Scalenus medius, Scalenus posterior \\
Semispinalis capitis, Semispinalis cervicis, Splenius capitis, Splenius cervicis \\
Sternocleidomastoid, Sternohyoid, Sternothyroid, Thyrohyoid
\end{tabular}

\section{Perturbed muscles}

Thoracic spine Iliocostalis thoracis (IT), Rotatores (ROT), Semispinalis thoracis (SST)

Serratus posterior superior (SPS), Spinalis thoracis (ST), Trapezius (TP)

Rhomboideus major (RMMA), Rhomboideus minor (RMMI), Serratus anterior (SA)

Subclavius (SCL)

Ribcage

Intercostales externi (IE), Intercostales interni (II), Levatores costarum (LEC)

Subcostales (SCO), Transversus thoracis (TT)

Lumbar spine

Iliocostalis lumborum (IL), Longissimus thoracis (LT), Multifidus (MF)

Obliquus externus abdominis (OEA), Obliquus internus abdominis (OIA), Psoas major (PM)

Quadratus lumborum (QL), Rectus abdominis (RA), Serratus posterior inferior (SPI)

Latissimus dorsi (LD)

sagittal plane, perturbations of the muscles residing at the right side were also reflected at the left side. Each location was perturbed $\pm 2.5 \mathrm{~mm}$ along the global $\left(\psi_{\text {global }}\right)+X^{g},+Y^{g}$, and $+Z^{g}$-axes.

We computed the relative changes (as the percent of the nominal value) in predicted compressive and shear forces at the middle-thoracic (T6/T7), thoracolumbar (T12/L1), and lumbar (L4/L5) discs due to the perturbations. The compression force was calculated along the $y^{j}$-axis of the intervertebral joint reference frame $\left(\psi_{\text {joint }}\right)$ while the anterior-posterior (AP) and medial-lateral (ML) shear forces were the components along the $x^{j}$ and $z^{j}$-axes, respectively (Fig. 5.1). The percent changes in the disc forces were computed for standing upright $\left(0^{\circ}\right), 30^{\circ} \& 60^{\circ}$ flexion, $15^{\circ} \& 30^{\circ}$ lateral bending, and $15^{\circ} \& 30^{\circ}$ axial rotation.

Similar to earlier studies in the lower extremity [51, 144], a metric-overall sensitivity index-was calculated for each perturbation. This index is essentially a measure of the combined reaction of all muscle elements. Overall sensitivity index (OSI) is calculated as the total percent change in the muscle momentum contributions by using Eq. 5.1

$$
O S I=\frac{\Sigma_{i} \int_{0}^{T}\left|F_{\text {perturbed }, i}^{m t}(t)-F_{\text {nominal }, i}^{m t}(t)\right| d t}{\Sigma_{i} \int_{0}^{T} F_{\text {nominal }, i}^{m t}(t) d t} \times 100 \%
$$


In this equation, $F_{\text {perturbed,i }}^{m t}(t)$ and $F_{\text {nominal,i }}^{m t}(t)$ stand for the perturbed and nominal muscle-tendon actuator forces (averages of the left and right sides, $i$ : muscle element) and $t$ is the duration between two consecutive solution steps $(t=1$ second and $T=10$ seconds). In Eq. 5.1. predicted forces due to perturbations were subtracted from the corresponding nominal values, and the differences were integrated over each time step. To calculate the percent change, the cumulative change in momentum was divided by the nominal cumulative momentum. To avoid zero denominators, nominal muscle forces were set to $0.1 \mathrm{~N}$ when smaller than $0.1 \mathrm{~N}$ ). Finally, grand maximal values of OSI were obtained for each muscle from their corresponding elements along six directions. OSIs were calculated for standing upright, flexion $\left(60^{\circ}\right)$, lateral bending $\left(30^{\circ}\right)$, and axial rotation $\left(30^{\circ}\right)$.

All analyses were run on a desktop computer with 32 cores of an Intel Xeon Gold 6130 (Intel Core, Intel, Santa Clara, CA) and 32 gigabytes of RAM. The simulations were batchprocessed and post-processed in Python 3.6 environment using AnyPyTools library [145].

\subsection{Results}

Changes in the compressive and shear disc forces due to perturbations were calculated for each trunk posture and are presented in Fig. 5.2 For each muscle, the maximal increases and decreases in force magnitudes were obtained for its attachment sites along six directions (positive and negative $+X^{g},+Y^{g}$, and $+Z^{g}$-axes). In this table, changes are emphasized by coloring, where red indicates relatively higher increases and blue does higher decreases. Furthermore, nominal disc reaction forces and the corresponding minimum and maximum values are given in Table 5.2 .

The perturbations in most of the muscle groups had only negligible effects (typically below $5 \%$ ) on predicted disc forces. However, the increases and decreases as high as $353 \%$ and $172 \%$ respectively were found for shear forces. These were $17 \%$ and $7 \%$ for the compression forces. Disc forces were most sensitive to perturbations in certain muscle groups located in the ribcage and lumbar regions. The perturbations in the psoas major attachments caused the largest increase in the compressive forces $(84 \mathrm{~N})$ at the L4/L5 joint during $30^{\circ}$ axial rotation, and longissimus thoracis and rotatores resulted in the largest decrease $(32 \mathrm{~N})$ at the $\mathrm{T} 12 / \mathrm{L} 1$ joint during $60^{\circ}$ flexion. Intercostales externi caused the highest increase in the shear forces $(45 \mathrm{~N})$ at the T6/T7 joint, and quadratus lumborum led to the highest decrease $(74 \mathrm{~N})$ at the $\mathrm{T} 12 / \mathrm{L} 1$ joint, both during $30^{\circ}$ axial rotation. When all the joints and postures were considered, on average, quadratus lumborum had the most influence on predicted disc forces, followed by intercostales interni, longissimus thoracis, and intercostales externi. Furthermore, disc forces at the L4/L5 joint were the most sensitive against muscle attachment sites, followed by T6/T7 and T12/L1 joints. Although higher force magnitude changes were found for the compressive forces, percent changes were generally higher for the shear forces 
Table 5.2: Nominal intervertebral disc reaction forces [N]. Minimum and maximum values due to perturbations are given inside the parentheses, respectively.

\begin{tabular}{|c|c|c|c|c|c|c|c|c|}
\hline & & Upright & $\begin{array}{l}\text { Flexion } \\
\left(30^{\circ}\right)\end{array}$ & $\begin{array}{l}\text { Flexion } \\
\left(60^{\circ}\right)\end{array}$ & $\begin{array}{l}\text { Lateral } \\
\text { bending } \\
\left(15^{\circ}\right)\end{array}$ & $\begin{array}{l}\text { Lateral } \\
\text { bending } \\
\left(30^{\circ}\right)\end{array}$ & $\begin{array}{l}\text { Axial } \\
\text { rotation } \\
\left(15^{\circ}\right)\end{array}$ & $\begin{array}{l}\text { Axial } \\
\text { rotation } \\
\left(30^{\circ}\right)\end{array}$ \\
\hline \multirow[t]{3}{*}{ T6/T7 } & AP shear & $8(-3,37)$ & $-49(-57,-41)$ & $-65(-84,-58)$ & $17(9,36)$ & $25(14,41)$ & $42(33,57)$ & $66(40,111)$ \\
\hline & Compression & $384(357,447)$ & $344(330,354)$ & $365(344,384)$ & $408(398,459)$ & $453(443,512)$ & $420(411,477)$ & $529(519,574)$ \\
\hline & ML shear & $0(0,0)$ & $0(0,0)$ & $0(0,0)$ & $-12(-20,-4)$ & $-30(-42,-26)$ & $-7(-16,1)$ & $-15(-52,11)$ \\
\hline \multirow[t]{3}{*}{ T12/L1 } & AP shear & $24(21,33)$ & $-66(-70,-62)$ & $-248(-256,-243)$ & $26(23,33)$ & $33(31,40)$ & $27(24,33)$ & $37(29,42)$ \\
\hline & Compression & $425(409,444)$ & $584(579,590)$ & $738(706,806)$ & $439(430,457)$ & $460(456,482)$ & $459(456,482)$ & $534(507,569)$ \\
\hline & ML shear & $0(0,0)$ & $0(0,0)$ & $0(0,0)$ & $18(11,20)$ & $29(20,32)$ & $6(20,32)$ & $4(2,13)$ \\
\hline \multirow[t]{3}{*}{ L4/L5 } & AP shear & $-294(-350,-263)$ & $-456(-463,-450)$ & $-592(-620,-576)$ & $-335(-496,-325)$ & $-440(-483,426)$ & $-322(-378,-3055)$ & $-352(-423,-337)$ \\
\hline & Compression & $503(477,546)$ & $821(815,828)$ & $1245(1213,1311)$ & $545(533,613)$ & $652(631,693)$ & $535(522,587)$ & $586(567,671)$ \\
\hline & ML shear & $0(0,0)$ & $0(0,0)$ & $0(0,0)$ & $-24(-27,-17)$ & $-43(-51,-33)$ & $22(18,25)$ & $41(37,45)$ \\
\hline
\end{tabular}

(anterior-posterior and medial-lateral). The computed disc forces were also influenced by trunk angle and posture but the effect differed between different muscle groups.

Grand OSI corresponding to each muscle is shown as stacked columns in Fig. 5.3. Similar to the changes in disc forces, relatively higher values were found for certain muscles (quadratus lumborum, intercostales interni, intercostales externi, longissimus thoracis, and iliocostalis lumborum) located in the ribcage and lumbar regions. For all other muscle groups, cumulative OSI was below $10 \%$.

\subsection{Discussion}

It was previously asserted that insecurities in data and assumptions embedded in musculoskeletal models of the spine, such as vertebral geometry, ligament material properties, and spinal movement, would affect predictions of internal forces. Zander et al. (2016) found that variations in the center of rotation of the intervertebral discs considerably affected muscle activities and disc forces in the lumbar spine [138]. Arshad et al. (2016) investigated the effects of using different spinal rhythms and intra-abdominal pressures [85]. They concluded that there were significant effects on disc and muscle forces and that these increased with trunk flexion. Putzer et al. (2016b) found that anatomical variations of vertebral body height, disc height, transverse process width, and the curvature of the lumbar spine greatly influenced the joint force predictions at the L4/L5 disc [48]. Recently, Ignasiak et al. (2016) found that modeling the thorax as a single lumped rigid body instead of a flexible structure resulted in moderately lower compressive disc forces but significantly changed muscle forces [40]. Furthermore, Nussbaum et al. (1995) studied the effects of inter-individual variations in muscle lines-of-action in a simple biomechanical model on muscle and joint forces at an erect posture under externally applied moments on the frontal and sagittal planes [140]. They found that predicted L3/L4 compression forces could change by more than $100 \mathrm{~N}$ while L3/L4 shear forces could change by more than $50 \mathrm{~N}$. 


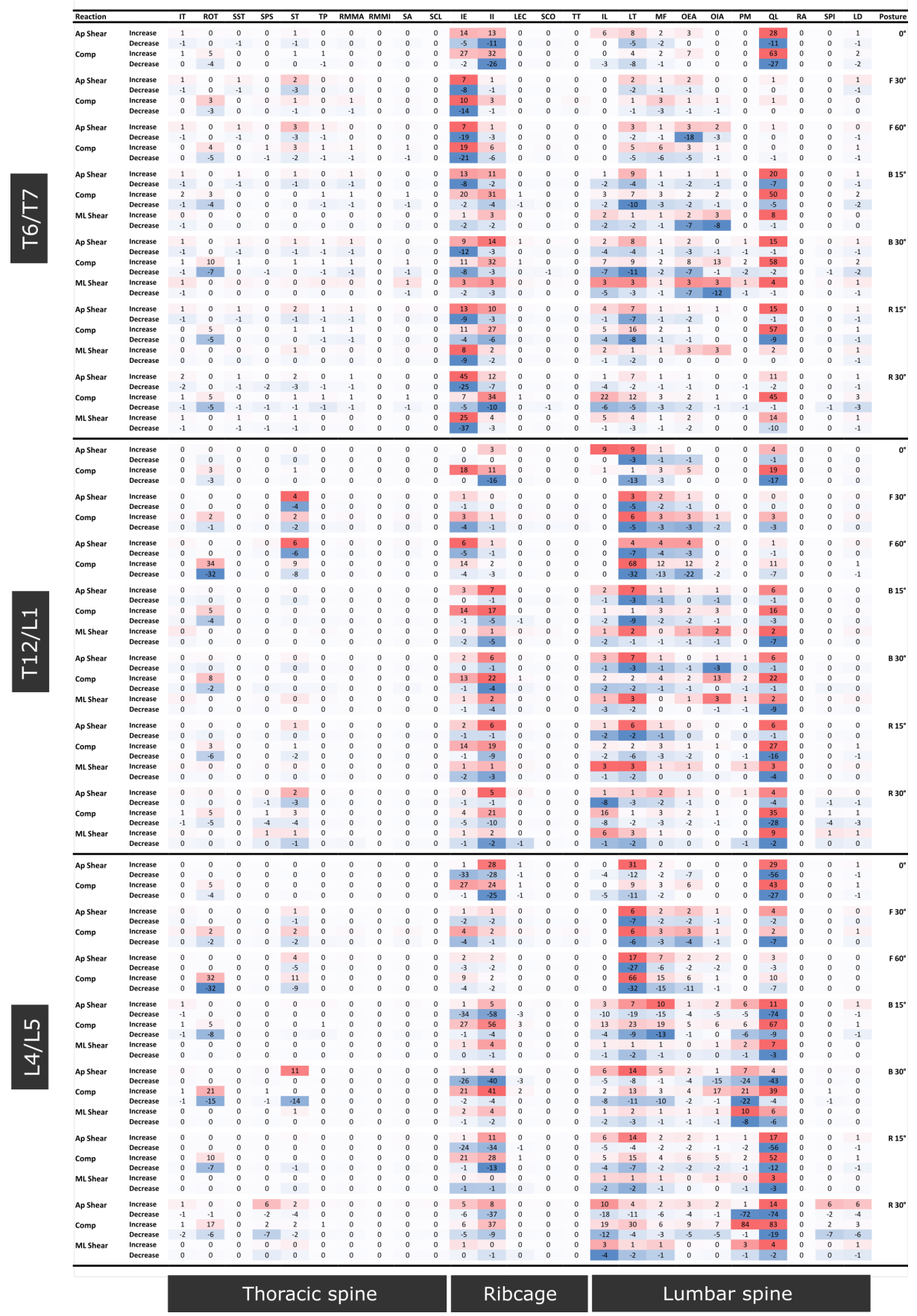

Figure 5.2: Changes in the compressive and shear forces at the T6/T7, T12/L1, and L4/L5 discs due to perturbations in muscle groups. These values were calculated at the following trunk postures: upright position $\left(0^{\circ}\right), 30^{\circ} \& 60^{\circ}$ flexion $\left(\mathrm{F} 30^{\circ} \& \mathrm{~F} 60^{\circ}\right), 15^{\circ} \& 30^{\circ}$ lateral bending $\left(\mathrm{B} 15^{\circ} \& \mathrm{~B} 30^{\circ}\right)$, and $15^{\circ} \& 30^{\circ}$ axial rotation $\left(\mathrm{R} 15^{\circ} \& \mathrm{R} 30^{\circ}\right)$. For each muscle, the maximal increases and decreases in force magnitudes were calculated for its attachment sites along six directions (positive and negative $+X^{g},+Y^{g}$, and $+Z^{g}$-axes). Larger changes are emphasized by coloring, where red indicates relatively higher increases and blue indicates higher decreases. 


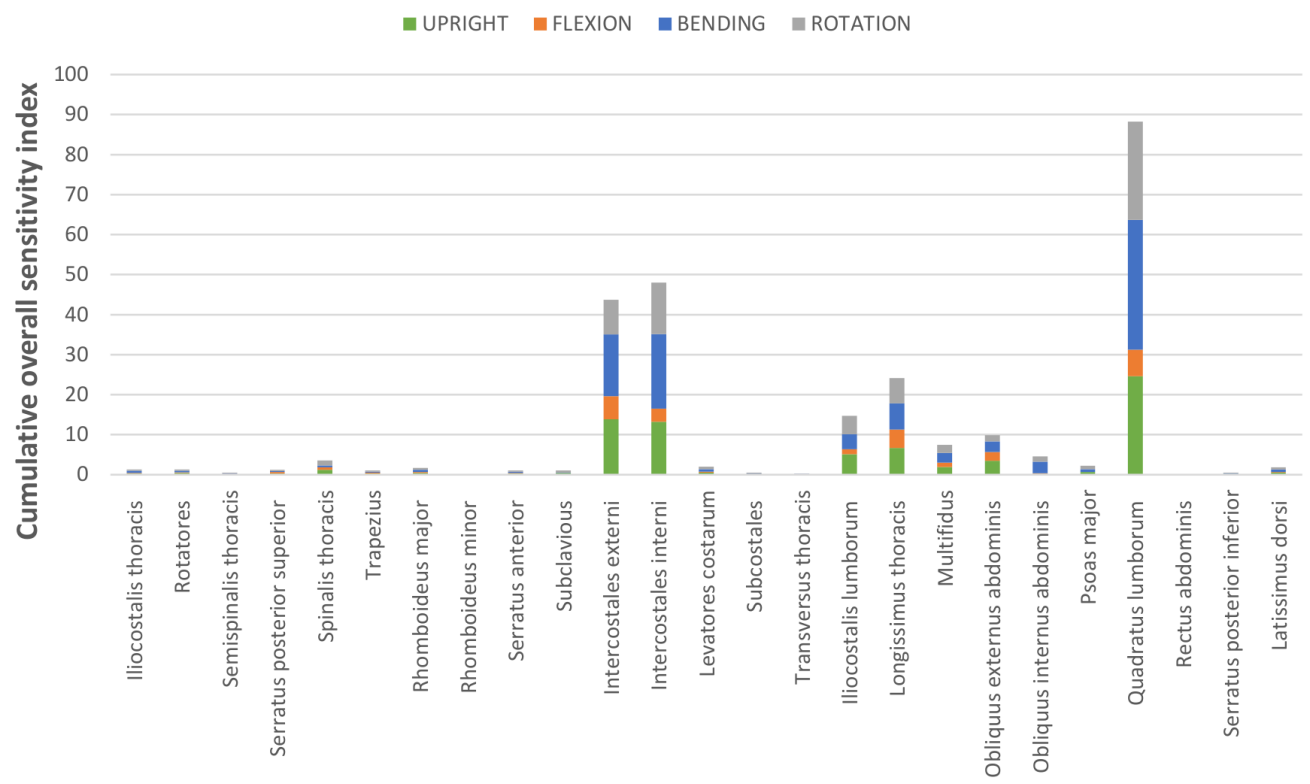

Muscles

Figure 5.3: Grand maximal values of OSI are shown as stacked column charts. In this depiction, the height of each bar corresponds to the sum of the maximal values for each muscle in each movement.

In the current study, we investigated the sensitivity of muscle and intervertebral disc force predictions to potential errors in modeling muscle attachment sites (muscle origin, insertion, and via points). For this, we used a previously validated model of the entire human spine [137], perturbed each attachment location $\pm 2.5 \mathrm{~mm}$ along six anatomical directions, and quantified the changes in muscle and disc forces during upright position, flexion, bending, and rotation of the trunk. Similar to previous studies [51, [144], we computed OSI for each perturbation. This index is a measure of the collective reaction of all muscles to maintain the joint moments. The error magnitude studied in this work can be introduced during the measurement of the attachment locations in cadaveric studies and their implementation in musculoskeletal models or due to inter-individual anatomical variations. Based on our experience in measuring and defining attachment locations of spinal muscles, this is a large but realistically possible error.

Our simulations indicated that small errors in modeling the muscle attachment sites caused slight to considerable changes in the computed disc forces (Fig. 5.2 and Table 5.2). Although for the majority of muscles the changes were minor (less than $5 \%$ ), for some muscles, for example, quadratus lumborum, the change in disc shear and compressive force was as high as $353 \%$ and $17 \%$, respectively. In general, we found that the percent changes in disc shear 
forces were higher for the changes in the muscle lines-of-action than the compression forces. This was attributed to the fact that disc compressive forces were much larger than disc shear forces. Thus, for the same absolute force change, we would observe larger percentile changes in shear forces. Furthermore, intervertebral disc forces at the L4/L5 joint were the most sensitive against muscle attachment locations, and the T6/T7 joint showed higher sensitivity than the T12/L1 joint.

Our analyses suggest that muscle recruitment patterns were most sensitive for the attachments of certain muscle groups positioned in the ribcage and lumbar spine (Fig. 5.3). These muscles were (in order of sensitivity) quadratus lumborum, intercostales interni, longissimus thoracis, and intercostales externi. These muscle groups have multiple elements that attach to several bones and contribute to the moments at the costo-sternal, compound costo-vertebral and costo-transverse, and lumbar intervertebral joints during trunk movements. These findings indicate that the modified moment arms made these muscles less suitable to contribute to these joint moments and favored other muscles for maintaining the needed joint moments [51, 144]. Similar to the changes in the disc forces, relatively higher sensitivities were found for these muscles. This indicates the dependence of spinal loads to muscle recruitment patterns and hence to muscle attachment locations. Other muscle groups had much lower sensitivity values implying that errors involved in modeling their locations would have a much smaller impact on muscle function and internal loads. Moreover, figure 5.3 depicts that the changes in the muscle forces were also dependent on the simulated tasks. The computation of muscle forces was most sensitive in trunk lateral bending followed by upright posture, trunk rotation, and trunk flexion. In a similar study, Nussbaum et al. (1995) elaborated that spinal forces were substantially altered for moderate changes in the lines-of-action of the oblique muscles but were minimally changed for the vertically oriented muscles [140]. We also found that disc forces were sensitive to external and internal oblique muscles as well as some vertically oriented muscles such as iliocostalis lumborum, longissimus thoracis, and quadratus lumborum. However, our analyses did not identify the oblique muscles as being most influential nor vertically oriented muscles as least influential. The discrepancies in the findings can perhaps be due to using different spinal morphology and modeling practices or to simplifications of the muscular anatomy.

Several (limiting) assumptions affect the findings of this study. Firstly, we used a simple muscle model which only took into account isometric strengths for modulating force exertion. We choose to not to represent force-length and force-velocity relationships as this would require further calibrating muscles' optimal fiber and tendon slack lengths for which there is no data. Secondly, the movement at the intervertebral disc articulations was simplified by using spherical joints which were constrained for translational DoFs. Ghezelbash et al. (2015) found low to moderate impact on model predictions and suggested that intervertebral translational DoFs can be ignored for small trunk angles in flexion [146]. 
Furthermore, Senteler et al. (2017) investigated the sensitivity of disc force predictions to center of rotation (COR) locations [134]. They asserted that a posterior COR in an upright position and an anterior COR in flexion would optimize the lumbar joint loads. Thirdly, we did not incorporate spinal ligaments but implemented their collective rotational stiffnesses linearly in the intervertebral and ribcage joints. Thus, excluding the ligaments or simplifying their non-linear mechanical behaviors could alter the load sharing mechanism between the muscles and affect our findings [135, [139]. For example, Putzer et al. (2016b) found that implementing stiffer ligaments could cause a shift of the loads to the lower lumbar disc levels [139]. Fourthly, the facet joints between the adjacent vertebrae were not modeled. It was previously discussed that these joints will decrease the forces carried through the anterior column during trunk extension [12, 33]. Extension was, however, not studied in this study. Fifthly, the data presented in this work was obtained by using a subject-specific model of the spine where anatomical variations in spinal geometry and muscle morphology were not accounted for. Previous studies found that the spinal curvature had a major influence on calculated internal loads [48, 86, 136]. Briggs et al. (2007) reported strong correlations between thoracic spine curvature and the vertebral loads and thoracic curvature and muscle forces [86]. They discussed that a higher kyphosis angle resulted in larger normalized flexion moments and the disc forces. Thus, generalizing our findings to other musculoskeletal representations of the spinal morphologies requires further study. Lastly, we used a frequently used optimization criterion which minimized muscle fatigue for muscle recruitment. Implementing a different performance criterion might affect the findings. Nevertheless, we do not feel these assumptions will alter the qualitative behavior of the model much.

The majority of the previous generic musculoskeletal models of the spine relied on the anatomical illustrations provided in the previous dissection studies for defining muscle lines-of-action. Our findings suggest that for most muscles this only moderately affects the results, but for some muscles this approach might lead to erroneous evaluation of the muscle function and spinal loads. We would thus recommend using anatomical datasets in which muscle attachment locations were measured precisely or using imaging methods to acquire muscle paths. In this line, certain care should also be taken when morphing an anatomical dataset for developing a personalized model of the spine, particularly for the muscles which affect spine biomechanics to a large extent as identified in this study.

\section{Disclosure statement}

None is required.

\section{Funding}

This study was supported by a research grant by fonds NutsOhra (1302-032). 
5 


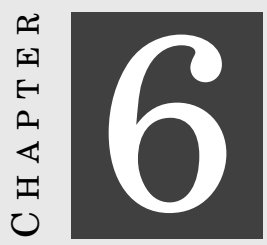

\title{
A COUPLED SET OF KINEMATIC AND ELECTROMYOGRAPHIC DATA FOR VALIDATION OF SPINAL MUSCULOSKELETAL MODELS
}

\begin{abstract}
Validation of internal spinal loads, as estimated by musculoskeletal models, is essential before use for clinical research. Surface electromyography is an indirect but noninvasive technique that can help in the validation process. In this study, we measured trunk muscle activities and movements simultaneously in fourteen healthy individuals during isometric and dynamic trunk exertions. Segmental rotations were calculated for measured spinal levels, and muscle activation patterns were investigated. Despite some gender and body height related differences in activation patterns, consistent muscle activation trends were found in most static and dynamic exertions. Measured trunk tasks can be isometrically or dynamically simulated in inverse dynamics based spinal musculoskeletal models, and predicted and measured muscle activations can then be compared to verify musculoskeletal predictions.
\end{abstract}

In preparation to be submitted as: Riza Bayoglu, Gerhard van den Noort, Nico Verdonschot, Bart Koopman, Jasper Homminga, "A coupled set of kinematic and electromyographic data for validation of spinal musculoskeletal models". 


\subsection{Introduction}

Computational models can elucidate the relations between locomotion, muscle function, and spinal loads [28, 34]. Muscle and joint forces estimated through musculoskeletal simulations of trunk movements can help in the treatment of neuromuscular and musculoskeletal disorders [1, 2]. An essential concern before applying such models to clinical practice is that their fidelity must be critically assessed by identifying any forms of error and uncertainty in the simulations and sensititivies to these uncertainties [147]. Commonly, surface electromyography (sEMG) is used to verify models' predictions of internal loading [4, 30, 33, 36, 109, 148]. In this scheme, measured trunk movement (quasi-static or dynamic) is simulated, and predicted muscle activations are compared to measured muscle activities. As spinal loads are regulated by the way we move our trunk [85], it is essential to implement subject-specific measurements of spinal kinematics and muscle activities together in such protocols.

Several researchers measured in vivo spine movement by using medical imaging or motion capture techniques. For example, the movements in the sagittal plane (flexion and extension) were quantified for the lumbar [122, 124, 149] and cervical spines [150, 151]. Similarly, vertebral movements in the frontal and transverse planes were measured for the lumbar and thoracic regions [123, 125, 126, 152-154]. Some authors used inertial sensors to investigate the movement coordination within the trunk under dynamic conditions [127, 155]. Previously, sEMG activities of the major torso and abdominal muscles were investigated for discriminating between healthy and pathological spines [14, 156, 157]. These studies showed that sEMG can be reliably and non-invasively used for assessing abnormal muscle function during physiological trunk movements. Although spinal kinematics and sEMG activities of major trunk muscles were simultaneously measured and used for validating specific models previously [30, 109, 148], the use of this data remained limited only to these models because measured spinal kinematics was not reported. Moreover, a further understanding of the muscle function among individuals with different body height and gender is essential for trend validations of the muscle activities predicted in spinal musculoskeletal models. Thus, the main objective of this study was to measure segment kinematics and muscle activations simultaneously and synchronized during physiological static and dynamic trunk movements. This coupled dataset will enable implementing spinal movement and muscle activations together for validation of spinal musculoskeletal models. Additionally, we aimed to assess potential differences in muscle coordination strategies among healthy individuals with different body height and gender.

\subsection{Material and methods}

Fourteen healthy participants volunteered for this study, and signed an informed consent form. A local ethical committee confirmed that no ethical approval was necessary for this 
study. The subjects were recruited from the university students and staff. They were selected and divided into three groups according to their body height and gender (see Table 6.1). The first group (short male) included males with an average body height of $1.63 \mathrm{~m}$ (range: 1.61 $1.66 \mathrm{~m}$ ). The second group (short female) consisted of females with an average body height of $1.62 \mathrm{~m}$ (range: $1.57-1.65 \mathrm{~m}$ ), and the third group (tall male) included male subjects with an average body height of $1.82 \mathrm{~m}$ (range: $1.74-1.95 \mathrm{~m}$ ). Furthermore, each subject met the following criteria to participate in this study. The inclusion criteria were such that the subject:

- was healthy with no history of disorder on the back and spine that can influence its movement,

- have a BMI between 17 and 25,

- fit within one of the aforementioned groups with regard to body height and gender.

The exclusion criteria were such that the subject:

- had an history of disorders related to spinal movement,

- was over weighted (BMI > 25),

- had extensively trained his back or abdominal muscles,

- was pregnant.
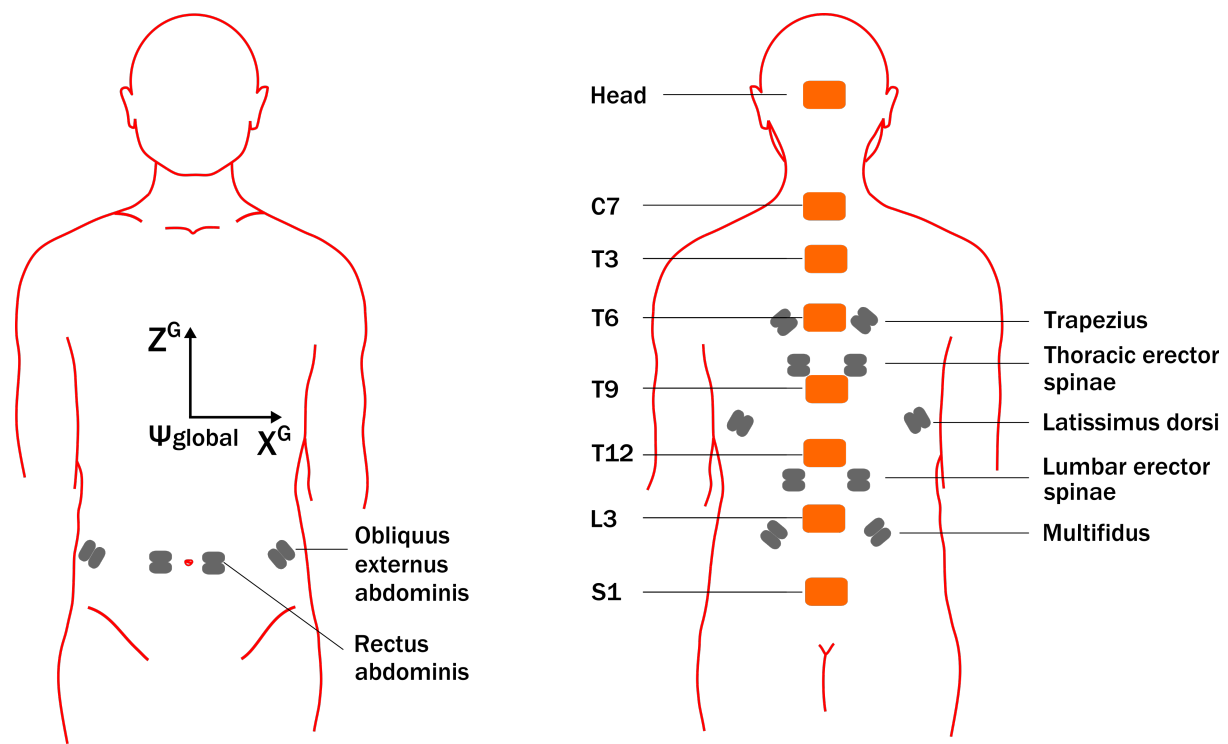

Figure 6.1: Locations and orientations of the sensors are depicted. Orange boxes indicate inertial measurement units (IMU), and EMG electrodes are displayed in gray. 
Table 6.1: Demographic features of the subject groups.

\begin{tabular}{|c|c|c|c|c|c|c|c|}
\hline Subject & Sex & Age & $\begin{array}{l}\text { Weight } \\
(\mathrm{kg})\end{array}$ & $\begin{array}{l}\text { Height } \\
\text { (m) }\end{array}$ & $\begin{array}{l}\mathrm{BMI} \\
\left(\mathrm{kg} / \mathrm{m}^{2}\right)\end{array}$ & $\begin{array}{l}\text { Trunk length } \\
\text { (m) }\end{array}$ & Group \\
\hline 1 & $\mathrm{~m}$ & 26 & 62 & 1.74 & 20 & 0.52 & 3 \\
\hline 2 & $\mathrm{~m}$ & 24 & 70 & 1.74 & 23 & 0.54 & 3 \\
\hline 3 & $\mathrm{f}$ & 23 & 59 & 1.64 & 22 & 0.49 & 2 \\
\hline 4 & $\mathrm{~m}$ & 21 & 74 & 1.84 & 22 & 0.55 & 3 \\
\hline 5 & $\mathrm{f}$ & 19 & 49 & 1.60 & 19 & 0.46 & 2 \\
\hline 6 & $\mathrm{~m}$ & 29 & 62 & 1.63 & 23 & 0.48 & 1 \\
\hline 7 & $\mathrm{~m}$ & 26 & 76 & 1.83 & 23 & 0.56 & 3 \\
\hline 8 & $\mathrm{~m}$ & 24 & 76 & 1.95 & 20 & 0.57 & 3 \\
\hline 9 & $\mathrm{f}$ & 29 & 44 & 1.57 & 18 & 0.44 & 2 \\
\hline 10 & $\mathrm{f}$ & 21 & 56 & 1.65 & 21 & 0.49 & 2 \\
\hline 11 & $\mathrm{~m}$ & 27 & 61 & 1.61 & 24 & 0.43 & 1 \\
\hline 12 & $\mathrm{~m}$ & 22 & 62 & 1.64 & 23 & 0.44 & 1 \\
\hline 13 & $\mathrm{f}$ & 23 & 45 & 1.60 & 18 & 0.41 & 2 \\
\hline 14 & $\mathrm{~m}$ & 33 & 62 & 1.66 & 22 & 0.53 & 1 \\
\hline All & & 24.8 (3.7) & $61.3(10.1)$ & $1.69(0.10)$ & $21.3(1.9)$ & $0.49(0.05)$ & \\
\hline Group 1 & & $26.0(4.6)$ & $61.7(0.5)$ & $1.63(0.02)$ & $23.3(0.4)$ & $0.45(0.05)$ & 1 \\
\hline Group 2 & & $23.0(3.7)$ & $52.0(6.7)$ & $1.62(0.03)$ & $19.9(1.8)$ & $0.47(0.03)$ & 2 \\
\hline Group 3 & & $24.2(2.2)$ & $71.6(5.9)$ & $1.82(0.09)$ & $21.6(1.4)$ & $0.55(0.02)$ & 3 \\
\hline
\end{tabular}

\subsubsection{Measurement of muscle activities}

Porti7 system (Twente Medical Systems International B.V., the Netherlands) was used for measuring electromyographic (EMG) activities of spinal and abdominal muscles. Bipolar snap-on $\mathrm{Ag} / \mathrm{AgCl}$ electrodes (42 $\mathrm{mm} \times 24 \mathrm{~mm}$, Covidien Kendall, Ireland) were used. In order to reduce movement artifacts in the signal, shielded cables were used to transfer raw EMG signals from the electrodes to the Porti device. The data was captured at a sample rate of $2048 \mathrm{~Hz}$. A graphical user interface was developed in MatLab 2017a (MathWorks, Inc., United States.) and used for recording and processing EMG signals.

Surface electromyography (sEMG) was applied bilaterally at seven locations (see Fig. 6.1). Following the guidelines provided mostly by the SENIAM project for placing the electrodes [158]:

1. Rectus abdominis: $4 \mathrm{~cm}$ lateral to the midline with the lower electrode at the level of the umbilicus.

2. Obliquus externus abdominis: above the anterior superior iliac spine, halfway between the iliac crest and the ribs, oriented at a slightly oblique angle.

3. Multifidus: along the line from the caudal tip of the posterior superior iliac spine to the interspace between L1 and L2 vertebrae at the level of L5 spinous process. 
4. Lumbar erector spinae: two fingers width lateral to the spinous process of L1 vertebra, orientated caudally.

5. Thoracic erector spinae: two fingers width lateral to the spinous process of $\mathrm{T} 8$ vertebra, orientated caudally.

6. Latisimus dorsi: lateral to the T9 vertebra, over the muscle belly and orientated slightly upwards.

7. Trapezius: at two-thirds of the line from the trigonum spinea to the T8 vertebra along a direction from the $\mathrm{T} 8$ vertebra to the acromion.

Maximum voluntary contraction (MVC) trials were done for normalization of the EMG signals. For this, subjects performed several MVC trials that specifically targeted abdominal and back muscles [159]. During these trials, subjects were verbally encouraged to apply their maximal effort. Each trial was repeated twice with 2 minutes of rest in between to prevent the muscle fatigue.

\subsubsection{Measurement of spinal movements}

We measured three-dimensional movements of the spine at several levels by using inertial measurement units (IMU) from Xsens Awinda (Xsens MTw, Xsens Technologies, the Netherlands). These IMUs ( $47 \mathrm{~mm}$ x $31 \mathrm{~mm}$ x $13 \mathrm{~mm}$ ) were secured to the subjects' skin by velcro hooks or a headband (Fig. 6.1): sacrum (S1), spinous processes of the L3, T12, T9, T6, T3, and C7 vertebrae, and the head [153]. Before placing the IMUs, important anatomical landmarks were palpated and marked with a whiteboard marker [160, Chapter 1].

Each inertial unit is equipped with a Kalman filter that combines the signals of triaxial accelerometers, magnetometers, and gyroscopes. The accuracy of these sensors was reported to be $0.3^{\circ}$ for the roll and pitch angles, and $0.73^{\circ}$ for the yaw angle [161]. The movement data were recorded at $100 \mathrm{~Hz}$ and was transferred to the Awinda station through a Bluetooth protocol. We used trigger signals to synchronize the signals measured through inertial units and EMG electrodes. The orientations of the IMUs are expressed in an Earth-fixed global reference frame. In this global frame, $Z$-axis is pointing upwards opposite to the direction of the gravitational acceleration (see Fig. 6.1). The $X$-axis is determined with the magnetic field of the earth, the positive $X$-axis is pointing in the direction of the magnetic North (laterally, to the left of the subject).

\subsubsection{Experimental tasks}

All experiments were performed under the guidance of the same researcher. Subjects performed static and dynamic tasks in three anatomical planes while we simultaneously 
measured the rotation of the spine and muscle activities. Each task was repeated three times. In each dynamic task, subjects started from a relaxed standing position, kept their arms at the sides, and moved in a self-paced manner to their maximum voluntary ranges of motion and subsequently back to the starting position. The following dynamic tasks were measured: trunk flexion, extension, lateral bending to the right and left, and axial rotation to the right and left. During static tasks, subjects kept certain trunk postures for ten seconds. The total trunk rotation was quantified from the sensor at the T12 level. This rotation is calculated as the change in orientation compared to the upright position and is referred to as the trunk angle. The following trunk postures were investigated: trunk flexion $\left(20^{\circ}, 40^{\circ}\right.$, and $\left.60^{\circ}\right)$, extension $\left(10^{\circ}\right.$ and $\left.20^{\circ}\right)$, lateral bending to both sides $\left(20^{\circ}\right.$ and $30^{\circ}$ ), and axial rotation to both sides $\left(15^{\circ}\right.$ and $\left.30^{\circ}\right)$.

\subsubsection{Data analysis}

EMG data was processed in MatLab 2017. Raw signals were high-pass filtered with a second order Butterworth filter with a cutoff frequency of $200 \mathrm{~Hz}$. Subsequently, signals were rectified and low-pass filtered at $6 \mathrm{~Hz}$ with a second order Butterworth filter. For each subject, signals from the MVC trials were grouped and stitched together to make one signal. A moving average filter was then applied with a window size of $500 \mathrm{~ms}$ to this signal for calculating maximal muscle activations. Afterward, the maximal values were used for normalizing the EMG signals measured during static and dynamic tasks.

The Cardan angles were calculated from the rotation matrices [127, 155]. According to the right hand rule, a positive angle around $X$-axis corresponds to flexion, a positive angle around $Y$-axis to lateral bending to the left, and a positive angle around $Z$-axis to axial rotation to the left.

\subsection{Results}

\subsubsection{Static tasks}

Muscle activities recorded during maintanences of the static tasks were calculated as percentages of the corresponding maximal values from MVC trials and are shown in Table 6.2 and Fig. 6.2, Fig. 6.3, and Fig. 6.4 In these figures, mean activations over all subjects were plotted, and error bars indicate one standard deviations. In the majority of the static and dynamic tasks, consistent muscle activation patterns were found.

In flexion, normalized EMG activations of rectus abdominis muscle nearly did not change with increased trunk angle (Fig. 6.2). Obliquus externus abdominis had slightly decreased activations compared to the upright position but almost unchanged activations during flexion. Activities of the right and left multifidus first increased until $40^{\circ}$ but later slightly 
decreased. Although smaller in magnitude, lumbar erector spinae muscle displayed similar activation trends as multifidus. Thoracic erector spinae, latissimus dorsi, and trapezius muscles had similar activation patterns which gradually increased with trunk flexion.
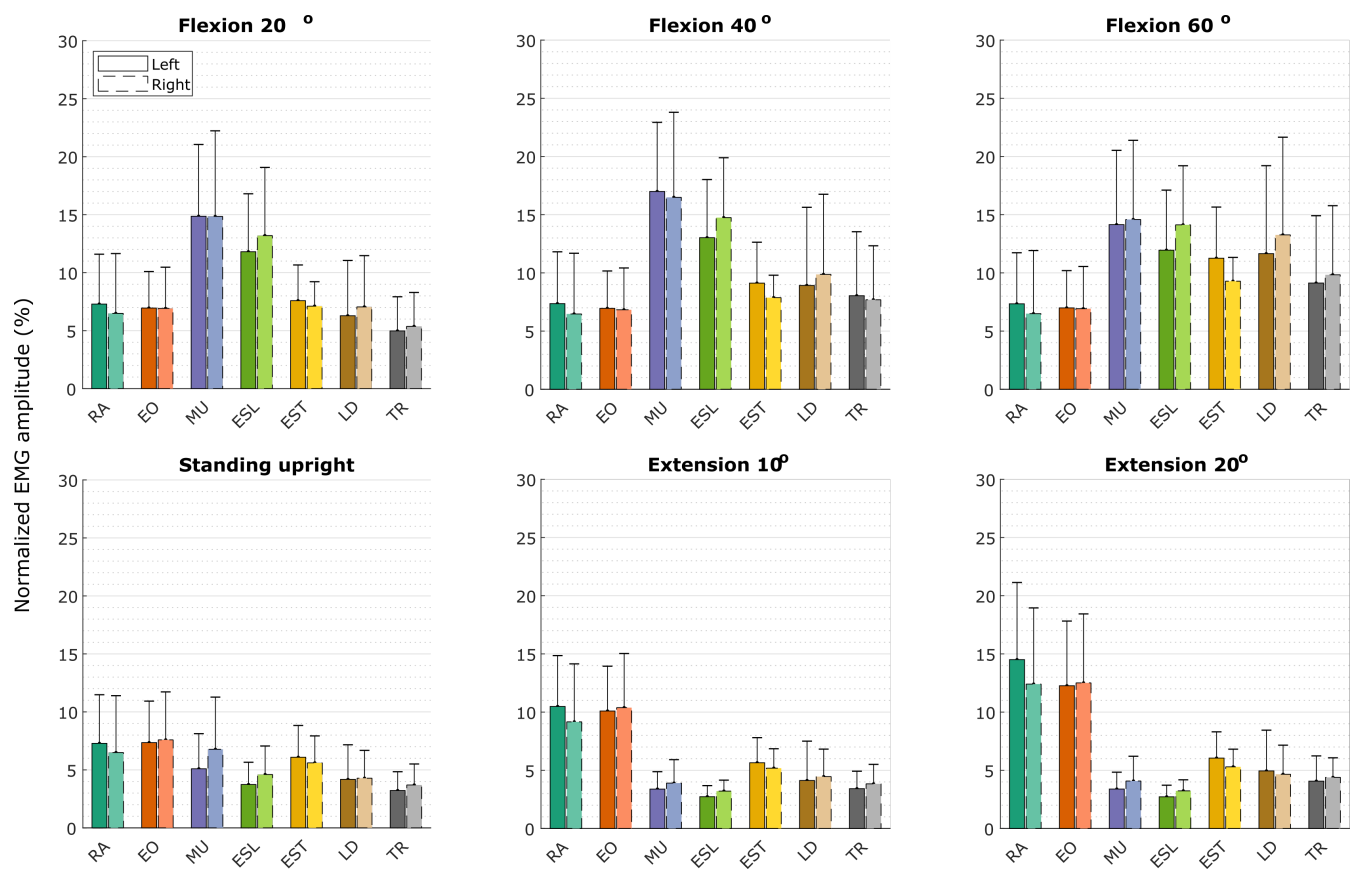

Figure 6.2: Mean MVC-normalized EMG activations during maintanences of the static postures: standing upright, flexion, and extension of the trunk. The error bars indicate one standard deviations. The abbreviations used for the muscle names are as follow: rectus abdominis (RA), obliquus externus abdominis (EO), multifidus (MU), lumbar erector spinae (ESL), thoracic erector spinae (EST), latissimus dorsi (LD), and trapezius (TR). 

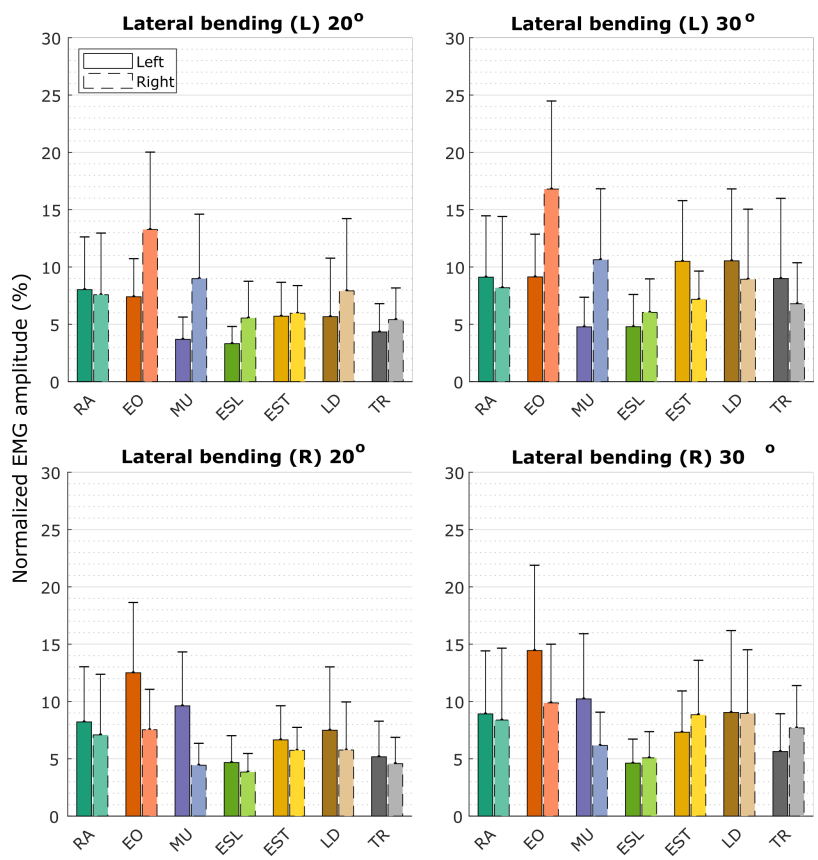

Figure 6.3: Mean MVC-normalized EMG activations during lateral bending to the left (L) and right $(\mathrm{R})$ sides.
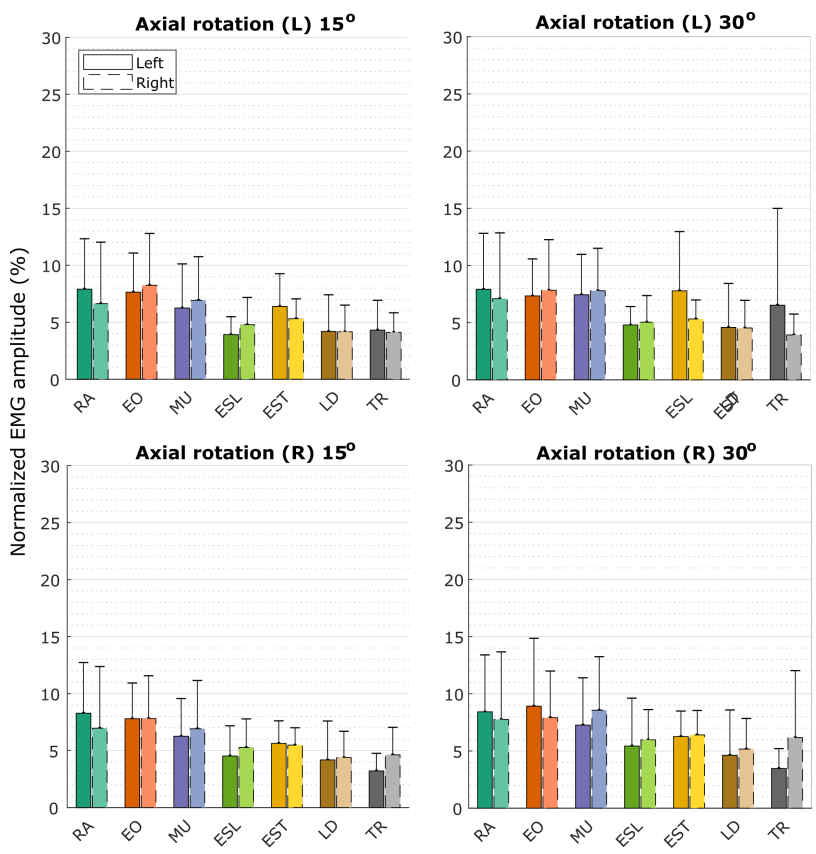

Figure 6.4: Mean MVC-normalized EMG activations during axial rotation to the left (L) and right $(R)$ sides. 
In extension, activities of the abdominal muscles gradually increased with trunk extension (Fig. 6.2). Multifidus and lumbar erector spinae muscles had moderately decreased activations during early extension but were practically constant afterwards. Thoracic erector spinae first had a slight decrease at $10^{\circ}$ and a slight increase at $20^{\circ}$. Latissimus dorsi and trapezius muscle activities slightly increased during extension.

In lateral bending, rectus abdominis muscle had moderately increased activations (Fig. 6.3. Contralateral obliquus externus abdominis were more active than the ipsilateral side, and their activations both increased with increasing trunk bending. Ipsilateral multifidus and erector spinae activations first decreased at $20^{\circ}$ but later increased at $30^{\circ}$. Contralateral multifidus and lumbar erector spinae presented much higher activations than the ipsilateral side having generally increasing activations during lateral bending. Activities of latissimus dorsi and trapezius muscles increased during lateral bending.

In axial rotation, rectus abdominis and latissimus dorsi muscles were active but had none to slight increases with axial rotation (Fig. 6.4. Contralateral obliquus externus abdominis muscle presented increased contraction state, while the ipsilateral side had nearly unchanged activation. Activities of multifidus, lumbar erector spinae, and trapezius muscles slightly increased. Thoracic erector spinae muscle did not show consistent activation trends. Ipsilateral trapezius had higher increases compared to the contralateral side during left and right axial rotations.

When three subject groups are compared, some variations in the EMG patterns were noted (see Table 6.3 and Table 6.4. In group 1 and group 3, obliquus externus abdominis muscle during flexion displayed practically similar activation levels compared to the upright position. In group 3 , the activity of thoracic erector spinae in extension nearly did not change compared to the upright position. Furthermore, in group 2, contralateral obliquus externus abdominis had decreased activity during axial rotation contrary to an increase when all the subjects are considered. 


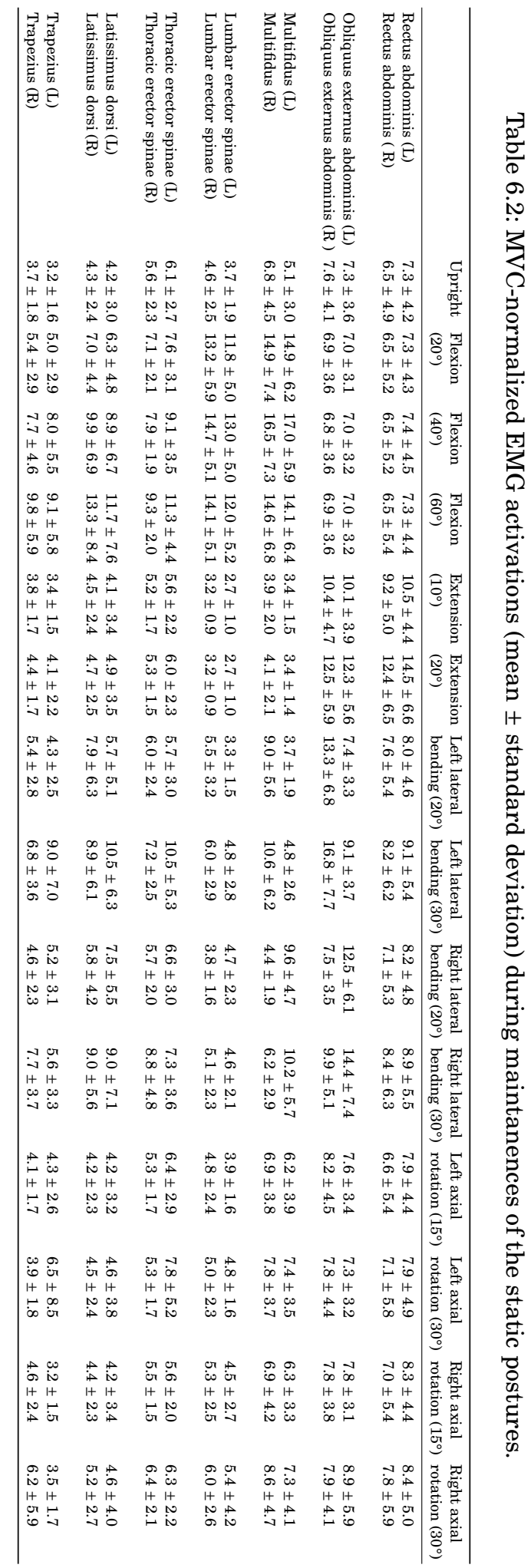




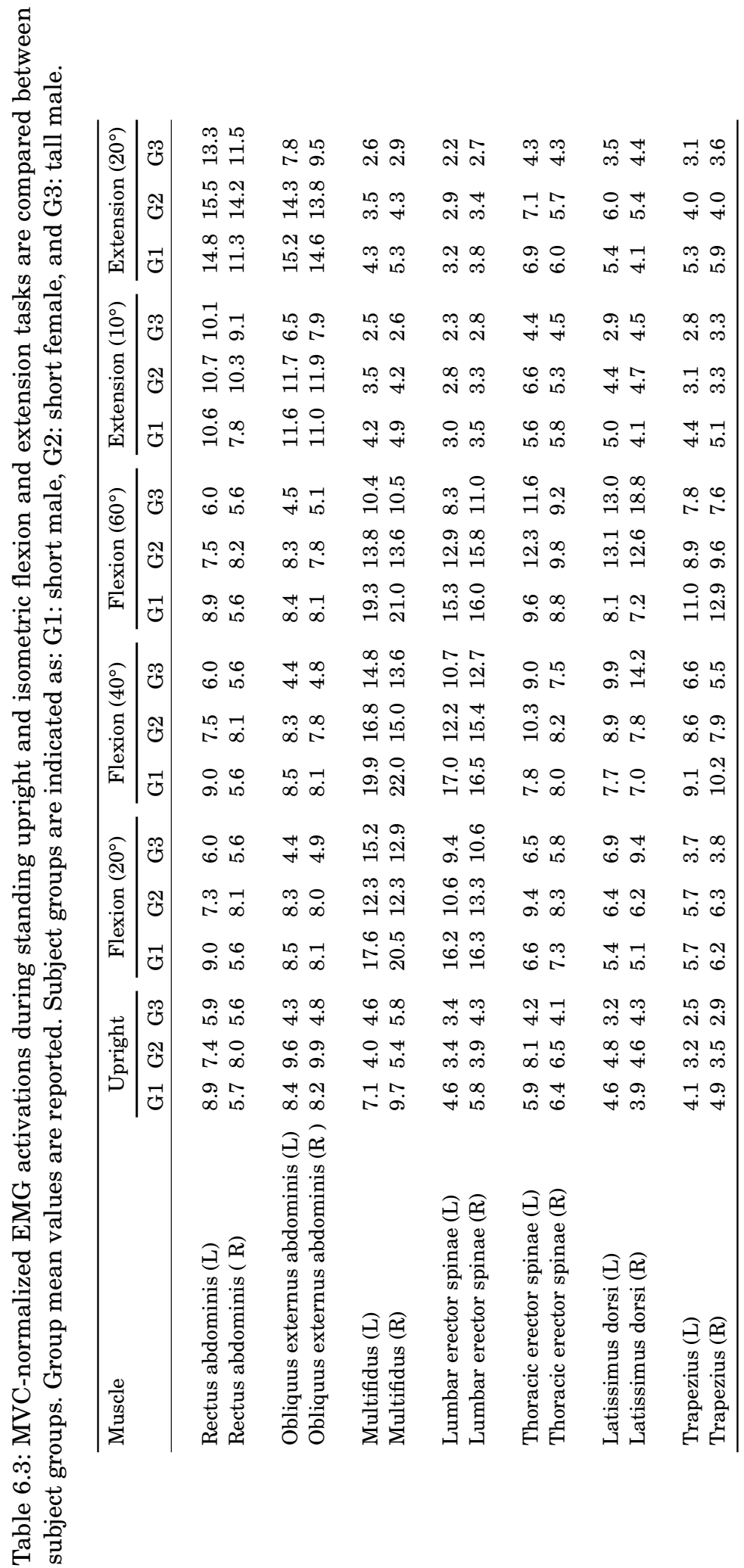




\subsubsection{Dynamic tasks}

Mean muscle activities measured during performances of the dynamics tasks were calculated as percentages of the corresponding maximal values (MVC) and are shown in Fig. 6.5, Fig. 6.6 and Fig. 6.7.

During early trunk flexion ( 25\%), multifidus and lumbar erector spinae muscles were more active than the other muscle groups (Fig. 6.5. Afterwards, their activities gradually decreased with further flexion. The activities of the thoracic spine muscles generally increased beyond early flexion. Abdominal muscles (particularly rectus abdominis) reached much higher activations towards the full range of movement.

Activities of the abdominal muscles gradually increased during trunk extension while lumbar region muscles had practically steady and negligible activations (Fig. 6.5). Thoracic muscle group had increasing contractions after around 30\% MVC.
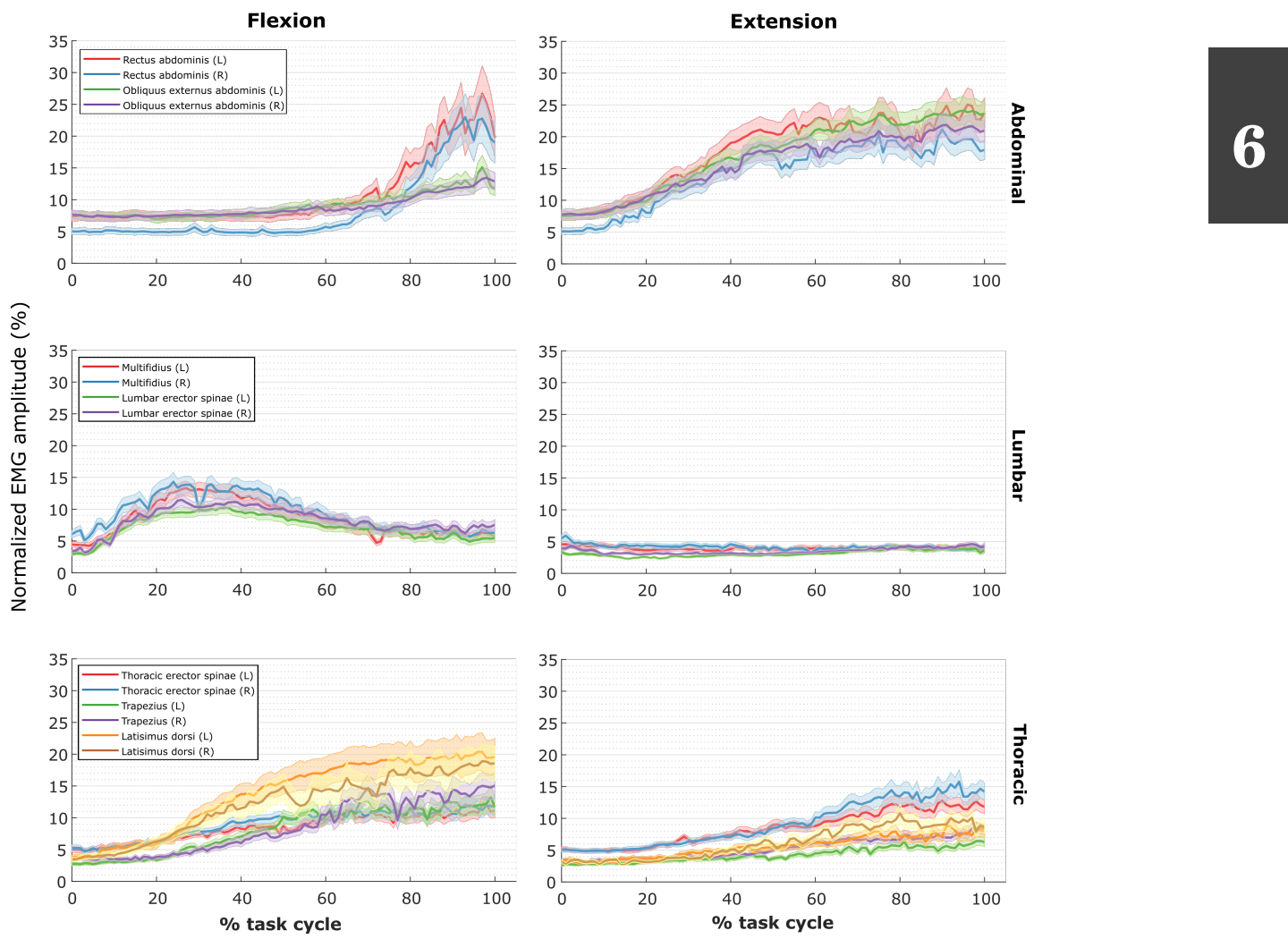

Figure 6.5: Mean MVC-normalized EMG activations during dynamic flexion and extension tasks. The horizontal axes represent percent task cycles. The shaded areas around the mean curves show the mean standard deviations. 
During lateral bending, activities of the right and left obliquus externus abdominis and left rectus abdominis muscles, in general, showed an increasing trend while right rectus abdominis had nearly constant activation (Fig. 6.6). Multifidus and ipsilateral lumbar erector spinae developed higher activations but contralateral lumbar erector spinae had nearly unchanged activation. Ipsilateral lumbar muscles had, in general, relatively higher activations than the contralateral sides beyond the mid-range. Ipsilateral thoracic muscles presented larger activations than the contralateral muscles, and the increases started around midway the full cycle.

During axial rotation of the trunk, ipsilateral abdominal muscles had higher increases than the contralateral muscles, and the activity of contralateral rectus abdominis almost did not change (Fig. 6.7). Lumbar muscles displayed increasing activation trends except for contralateral lumbar erector spinae which had nearly constant activation level during the full cycle. Similar to lateral bending, activities of ipsilateral thoracic muscles were higher

Lateral bending $(L)$
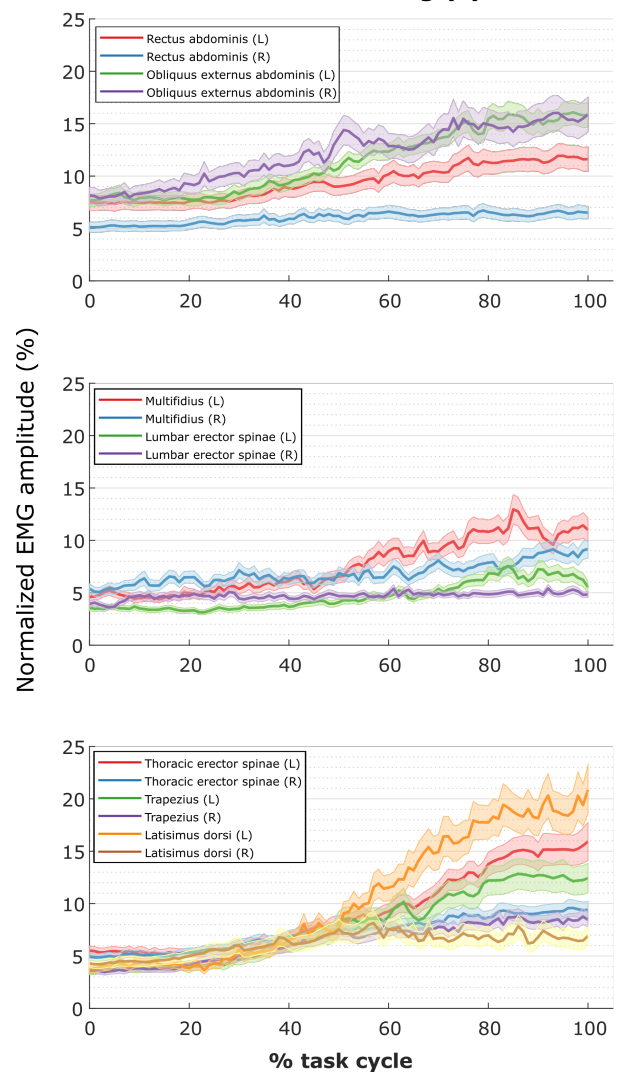
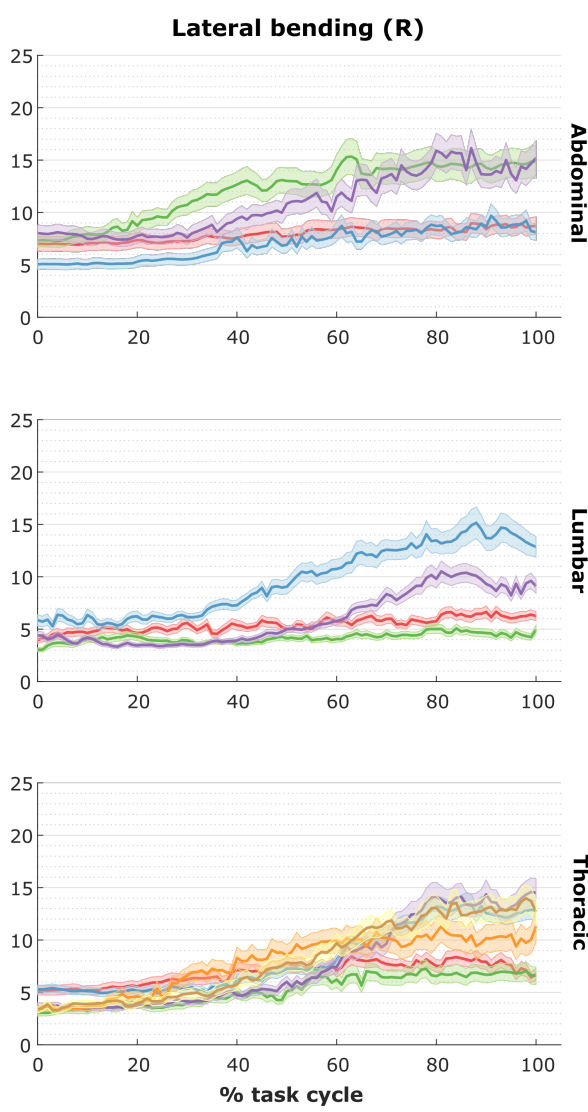

Figure 6.6: Mean MVC-normalized EMG activations during dynamic lateral bending tasks. The horizontal axes represent percent task cycles. The shaded areas around the mean curves show the mean standard deviations. 

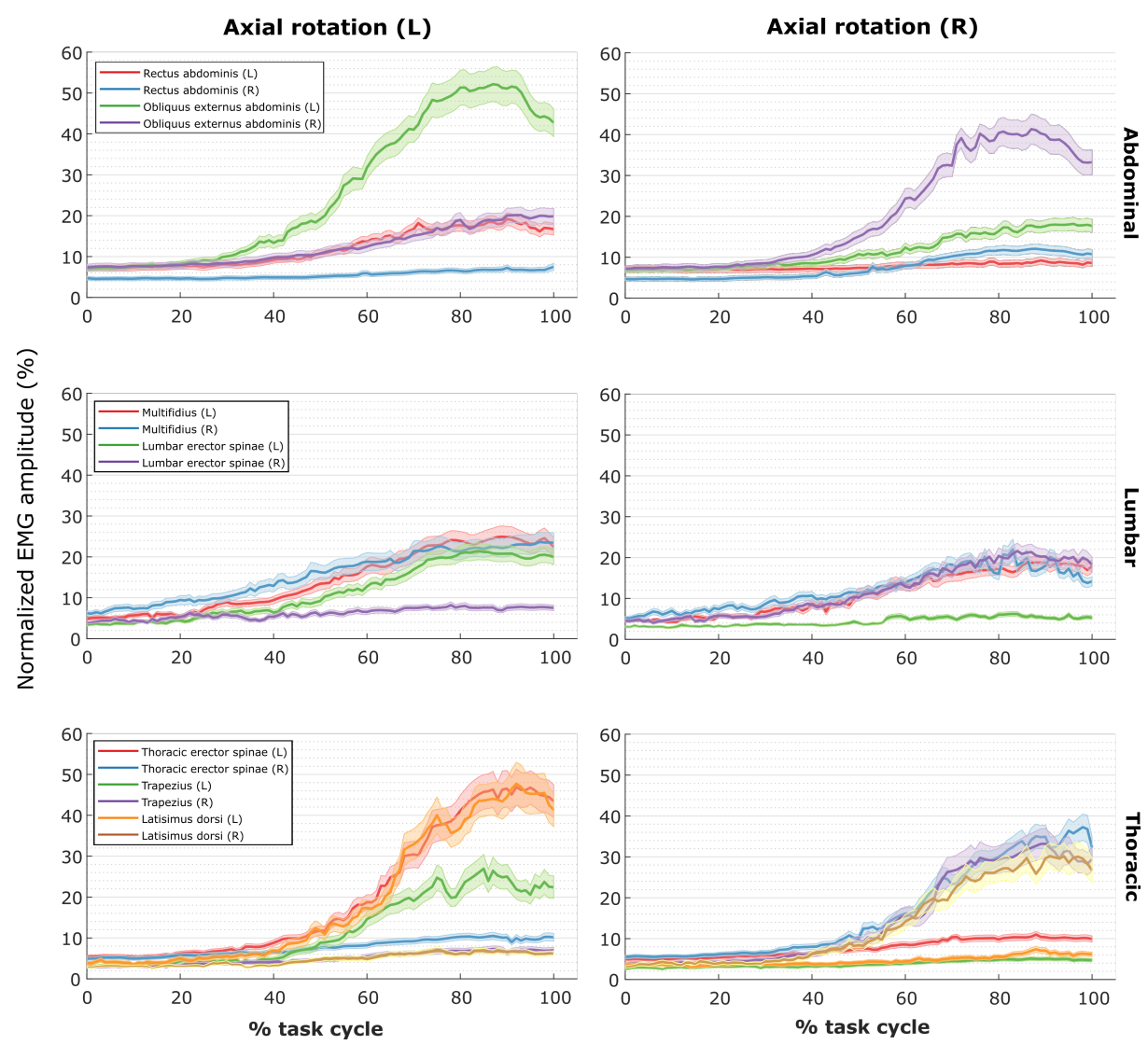

Figure 6.7: Mean MVC-normalized EMG activations during dynamic axial rotation tasks. The horizontal axes represent percent task cycles. The shaded areas around the mean curves show the mean standard deviations.

than the contralateral sides which had similar exertion levels as in the upright posture.

In group 1 (short male), compared to tall male and short female, the abdominal muscle activities started to increase after $60 \%$ of the task cycle, and lumbar muscles had fluctuations between $20 \%$ and $60 \%$ cycle during extension.

\subsection{Discussion}

In this study, we found that studied trunk movements resulted from complex interactions of various muscle groups. In most static and dynamic exertions, consistent muscle activation trends were noted. Lumbar and thoracic muscles were active during early and late trunk flexion respectively (Fig.6.5). Abdominal muscles presented higher activations mainly when close to maximum ranges of movement in flexion. In extension, thoracic and abdominal muscles were primarily active while multifidus and lumbar erector spinae presented almost no 
activity. Obliquus externus abdominis and multifidus muscles bilaterally and lumbar erector spinae ipsilaterally contributed to lateral bending in the frontal plane (Fig. 6.6). Ipsilateral thoracic muscles had higher activations than the contralateral muscles, and the contractions started to increase mid-lateral bending. During axial rotation, ipsilateral abdominal and thoracic muscles displayed much higher activity compared to contralateral muscles (Fig. 6.7). Starting early axial rotation, bilateral multifidus and ipsilateral lumbar erector spinae muscles had relatively higher contractions than in the upright position. These activation patterns of the back and abdominal muscles are largely in agreement with previous detailed investigations which used surface or needle EMG techniques [14, 156, 157, 162-164]. Our findings, however, contradict with a previous study which found higher activation levels in contralateral obliquus externus abdominis muscle during dynamic axial rotations from sitting position [14]. Also for isometric axial rotation, we found that the contralateral muscle had relatively higher activation than the ipsilateral side, which was in agreement with another study [157]. The discrepancy for the dynamic case might result from crosstalk from the obliquus internus abdominis muscle during intermediate to maximum axial rotation or from the differences in the experimental procedure.

During isometric flexion, in the short male and tall male groups, the activity of obliquus externus abdominis muscle almost did not change compared to the upright position whereas short female group had slightly decreased activations. In isometric axial rotation, contralateral obliquus externus abdominis in short female group had decreased activation, unlike male groups which had increased activations. Furthermore, the activities of rectus abdominis and obliquus externus abdominis muscles in short male group had increases only beyond $60 \%$ of the range of movement in dynamic extension. Multifidus and lumbar erector spinae muscles in this group had first an increase and later a decrease in activation contrary to short female and tall male groups where these muscles had nearly constant activations. These findings indicate gender and body height related differences in muscle activation patterns, particularly for obliquus externus abdominis muscle. Thus, we suggest that these factors should be considered when using muscle activation trends in validating musculoskeletal predictions of internal loads. However, a further research on larger subject groups is needed to assess the significance of these findings.

This study had some limitations. Firstly, the limited number of subjects (fourteen) that volunteered for this study hinders assessing the significance of the EMG findings, particularly for the variations due to differences with respect to gender and body height. Secondly, interpretations of the normalized EMG activities for the dynamic tasks should be done carefully. Soft tissue artifacts and muscle cross talks might be present in kinematic data and EMG, especially for large trunk movements. These artifacts might also affect our findings for the isometric exertions but likely to a lesser extent. Furthermore, normalizing the EMG signals with respect to time for the dynamic cases has its limitations [165]. Subjects were 
instructed to move on their self-paces to achieve maximum ranges of movement, which resulted in inter-subject variabilities for the durations of the movements. This also meant that the timing of activation was variable between repeated trials and different subjects. It is expected that sudden amplitude changes present for individual subjects would result in much smoother signals due to time normalizations of the average values across the subjects.

Knowledge of muscle coordination strategies that are employed during trunk movements provides an indirect but non-invasive option to validate musculoskeletal predictions [147]. Although activation trends of certain trunk muscles have been investigated previously, the applicability of those findings to model validation schemes remained limited since the geometry of movement was not measured nor reported on in earlier studies [30, 109, 148, 157]. The existence of mixed results in the literature as to activation patterns can be ascribed to inter-individual differences in muscle coordination strategies as well as the subject-specific factors [157]. In an attempt to further understand complex relationships between muscle function and locomotion, we measured back and abdominal muscle activities and trunk movements simultaneously during isometric and dynamic exertions. Measured trunk tasks can be isometrically or dynamically simulated in inverse dynamics based spinal musculoskeletal models, and predicted and measured muscle activations can then be compared to validate musculoskeletal predictions.

\section{Acknowledgements}

We thankfully acknowledge the financial support by fonds NutsOhra (1302-032). We also thank the Biomedical Signals and Systems Group at the University of Twente for providing us with the inertial measurement systems and sharing their help and experience. 


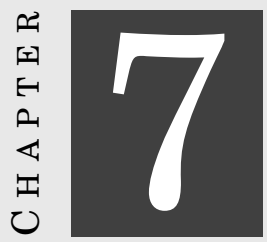

COMBINING MUSCULOSKELETAL AND FINITE ELEMENT MODELING TO PREDICT SPINAL FAILURE UNDER PHYSIOLOGICAL LOAD CASES. AN EXPLORATORY STUDY

Karlijn H.J. Groenen, Riza Bayoglu, Yvette M. van der Linden, Christopher P. Cop, Petra M. Braam, Dennis Janssen, Nico Verdonschot, Esther Tanck 


\subsection{Introduction}

When spinal bone metastases in patients with cancer progress, there is a risk of developing pathological fractures with pain and possibly also neurological injury becoming clinically evident. Since such adverse events dramatically affect the quality of life of the patient, they should be prevented as much as possible [166-168]. Preventing skeletal complications requires an accurate assessment of the fracture risk. Due to the absence of validated guidelines or an established predefined set of risk factors, however, it is at the moment unfeasible for clinicians to assess the fracture risk [169].

Quantitative computed tomography (QCT)-based case-specific finite element (FE) models show great promise to improve these fracture risk predictions. Previous work has demonstrated that FE models comprising single vertebrae can provide accurate predictions of vertebral stiffness and strength, when compared to in vitro experiments [170-175]. In this thesis we developed an FE model consisting of three consecutive vertebrae with corresponding discs (3-segment spinal unit), thereby providing a more realistic anatomical configuration with load bearing discs and vertebral bodies above and below the vertebral body of interest.

When assessing fracture risk not only bone strength should be considered, but also the loads applied to the bone should be taken into account. Current FE models on failure behavior mostly use fairly simple loading conditions, for example resembling upright standing, mainly to simulate experimental loading conditions in order to validate the models. Such simplified load cases, however, do not resemble the loads imposed on the spine during activities in daily life and might, therefore, be suboptimal for in vivo fracture risk prediction. In fact, loading scenarios such as forward flexion, lateral bending, or axial rotation, have demonstrated to affect the risk of fracture initiation in metastatically affected vertebrae [176].

The in vivo loads acting on the spinal column are a complex combination of joint contact forces, ligament forces, and muscle forces. Hence, incorporating joint contact forces, ligament forces, and muscle forces into the loading profile results in a more physiological loading condition. These forces can be estimated using musculoskeletal modeling. Musculoskeletal models apply mechanical principles to a system composed of bones, muscles, and ligaments in order to calculate joint contact forces resulting from gravitational force and the forces produced by the muscles and ligaments. As such, the load imposed on the spine during daily activities can be determined. Applying these sophisticated loading regimes to patientspecific FE models could provide more physiological fracture predictions.

In this exploratory study, we applied the workflow for generating FE models of 3-segment spinal units to a first patient case [177]. We combined musculoskeletal modeling and FE 
modeling and aimed to demonstrate that modeling physiological load cases instead of a simple loading regime can significantly affect failure predictions by patient-specific $\mathrm{FE}$ models in a patient with spinal bone metastases.

\subsection{Methods}

\subsubsection{Prospective patient study}

The Department of Radiotherapy (Radboud university medical center) conducts a randomized prospective study in which patients with symptomatic spinal bone metastases are randomized between conventional radiotherapy ( 1 x 8 Gy) or stereotactic radiotherapy (1 x $20 \mathrm{~Gy}$ ) to relieve pain (palliative treatment) (Principal Investigator: dr. Petra Braam, RACOST, ClinicalTrials.gov Identifier: NCT02407795) [12]. Ethical approval was obtained by the local medical ethical committee. Patients with a maximum of two consecutive or noncontiguous vertebrae involved by tumor, no or mild neurological signs, and a life expectancy of $>6$ weeks are included in this study. Patients with spinal instability or neurological deficit resulting from bony compression of neural structures, or a pathological or impending fracture needing surgical fixation are not eligible for inclusion. Further in- and exclusion criteria are depicted in Table 7.1

Before and three months after radiotherapy treatment patients undergo QCT and magnetic resonance imaging (MRI) scanning. In addition, patients fill out questionnaires on physical activity (IPAQ), quality of life (EORTC QLQ-C15-PAL and EORTC QLQ-BM22), and pain (BPI), but these are not included in the current exploratory study. Through their medical records, the patients are followed until either a fracture occurs, or until death, as competing risk, whichever occurs first. The occurrence of a (radiological) vertebral fracture or collapse is confirmed using CT and/or MRI scans.

\subsubsection{Patient case}

For this exploratory study, our department collaborates with the RACOST study team to study the predictive power of the FE model. We selected one patient from the patient study (female; 64 years at inclusion, body weight: $69 \mathrm{~kg}$; body height: $179 \mathrm{~cm}$; primary tumor: melanoma). This patient had an osteolytic lesion predominantly in the anterior border of the fourth lumbar (L4) vertebral body (Fig. 7.1). Approximately 50\% of the vertebral body was affected by tumor tissue. Pre-treatment, alignment of the spine was normal and there was no collapse of the vertebral body. The posterior elements of the spine were not affected by metastatic tissue. Three months after radiotherapy ( 1 x 20 Gy), radiological evaluation demonstrated a minor collapse of the L4 endplate (Fig. 7.1). At that time, the patient did not report symptoms indicating this collapse. However, 11 months after treatment, the patient's 
Table 7.1: In- and exclusion criteria.

\section{Inclusion criteria}

- Histologically proven solid tumor with radiological diagnosis of spinal metastases

- Pain score minimum 2 on 11 -point scale $(0=$ no pain to $10=$ worst imaginable pain $)$

- Maximum of 2 consecutive or noncontiguous vertebrae involved by tumor at current level of interest

- No or mild neurological signs (radiculopathy, dermatomal sensory change, and decreased

muscle strength of involved extremity)

- Karnofsky performance status $\geq 60$

- World Health Organization performance status $\geq 2$

- Life expectancy $>6$ weeks

- Age $\geq 18$ years

- Non-pregnant, non-lactating female patients. Sexually active patients of childbearing potential must implement effective contraceptive practices during study.

- Written informed consent

\section{Exclusion criteria}

- History of previous radiotherapy to the spine at the current level of interest or overlapping location

- Spinal instability or neurological deficit resulting from bony compression of neural structures

- Pathological fracture or impending fracture needing surgical fixation

- Prior surgery to the spine at the current level of interest or overlapping location

- Clinical signs of spinal cord compression or severe neurological deficits

- Patients with a pacemaker such that MRI cannot be performed or the treatment cannot be delivered safely

- Patients not able to undergo MRI

- Earlier nuclear medicine treatment, for example strontium 89 treatment

- Pregnancy

- Altered mental status that would prohibit the understanding and giving of informed consent

pain severely increased. Subsequent radiographs revealed a further increased collapse of the L4 endplate (reported as being a pathological fracture) as well as ventral and dorsal retropulsion of bony fragments, presumably also in the spinal canal (Fig. 7.1). At this point, the patient had no sign of neurological compromise.

\subsubsection{Obtaining loading conditions using musculoskeletal modeling}

We used the Twente Spine Model to predict compression and shear forces at the intervertebral joints during physiological movements [137]. In brief, the Twente Spine Model is a complete and coherent musculoskeletal model (MSM) of the entire human spine and is based on two anatomical datasets obtained from one embalmed cadaver (male; 79 years; height: $154 \mathrm{~cm}$; mass: $51 \mathrm{~kg}$ [ [84, 105]. In this left-right symmetrical MSM, bones were idealized as rigid body segments and muscle-tendon elements as force actuators (Fig. 7.2). A local reference frame for each vertebra was established. The origin of the local reference frame was located at the centroid of the vertebral body and the reference axes were based on vertebral landmarks [110]. The model uses the inverse dynamics method to predict muscle and joint forces based on an optimization function which minimizes the muscle fatigue. 

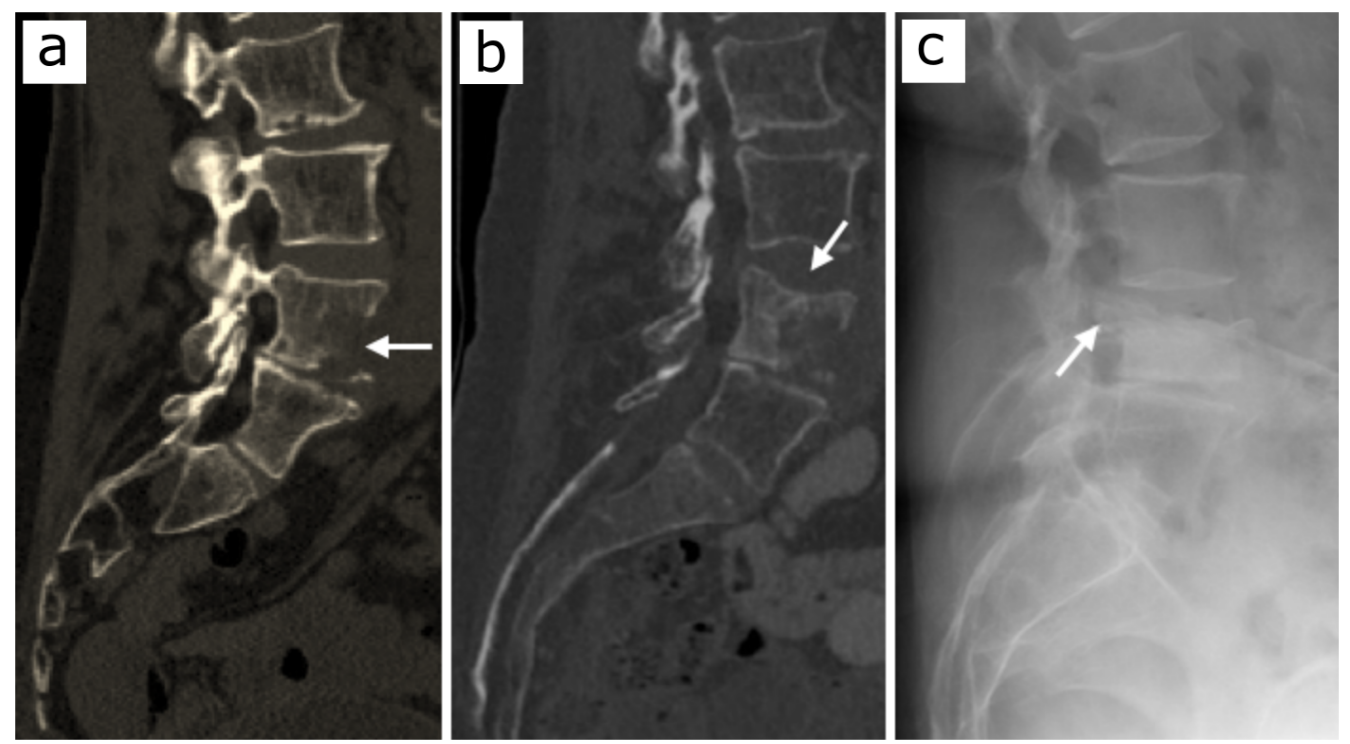

Figure 7.1: Medical imaging of the metastatically affected fourth lumbar (L4) vertebra. (a) CT scan pre-treatment showing the lytic lesion (arrow); (b) CT scan three months after radiotherapy treatment showing a minor collapse of the L4 endplate (arrow); (c) X-ray 11 months after treatment showing a pathological fracture as well as ventral and dorsal (arrow) retropulsion of bony fragments.

In total, 351 muscle-tendon elements (per body side) were included for 49 muscles. Intervertebral articulations were modeled as spherical joints with the center of rotation based on literature [111]. In the MSM we modeled the stiffness provided by the discs, ligaments, and the facet joints using linear torsion springs in three directions and applied these to the intervertebral joints.

To enable more realistic comparisons between the MSM and FE model, we scaled the MSM's body height and weight to represent the patient presented in this study. To obtain a scaled MSM, the spinal geometry (bone geometry, joint, and muscle attachment locations) was scaled linearly in three dimensions based on body height ratios. In addition, physiological cross sectional areas (PCSA) of the muscles were scaled based on the strength scaling factor (subject weight / model weight) $)^{2 / 3}$.

We simulated several quasi-static tasks with the scaled MSM. Each task started from the upright standing position and finished when the prescribed movement was achieved. We only defined the motion between $\mathrm{T} 1$ and pelvis segments and partitioned the movement accordingly. In addition, the total trunk motion was distributed between the pelvis and thoracic and lumbar regions of the spine. The following maneuvers were simulated: 


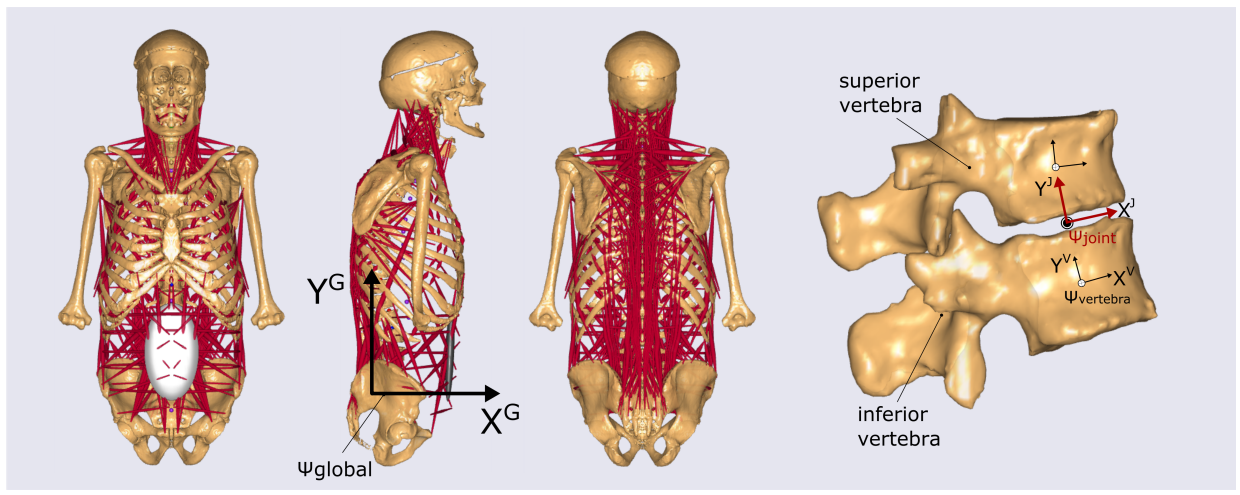

Figure 7.2: The musculoskeletal model (the Twente Spine Model [137]) used in this study, bones are displayed in yellow and muscle-tendon elements in red. The reference frame shown in the sagittal view is the global reference frame ( $\left.\psi_{\text {global }}\right)$. Vertebral reference frame $\left(\psi_{\text {vertebra }}\right)$ and (intervertebral) joint reference frame $\left(\psi_{\text {joint }}\right)$ are illustrated for one motion segment. The intervertebral compression force is calculated along the y-axis of the joint reference frame.

- Flexion $\left(45^{\circ}\right.$ and $\left.90^{\circ}\right)$

- Extension $\left(15^{\circ}\right)$

- Right lateral bending $\left(15^{\circ}\right)$

- Left lateral bending $\left(15^{\circ}\right)$

The resultant joint reaction forces and the positions of the L2/L3 joint during these maneuvers were calculated with respect to the L5 local reference frame.

\subsubsection{FE model generation}

An FE model of the patient's L3-L5 spine was generated using the workflow for generating case-specific FE models of 3-segment spinal units described previously [177]. The patient's pre-treatment QCT scan was used as input for the FE model. In short, quantitative computed tomography (QCT) images were retrieved (scan settings: $120 \mathrm{kVp}, 300 \mathrm{mAs}$, slice thickness $1.0 \mathrm{~mm}$, pitch 0.938 , standard reconstruction, in plane resolution 0.977 $\mathrm{mm}$ ). Geometric information for the vertebral bone and intervertebral discs (IVDs) was retrieved by segmenting the QCT images (Materialise Mimics 14.0, Materialise NV, Leuven, Belgium). The IVD was discretized into a nucleus pulposus (NP) and annulus fibrosis (AF), with the NP area constituting approximately $40 \%$ of the total disc area [178, 179]. The lesion was segmented by an experienced musculoskeletal radiologist. Vertebrae, lesion, and discs were converted into a tetrahedral solid mesh (HyperMesh 2017.1, HyperWorks, Altair Engineering, Inc. Troy, Michigan, United States). 
The patient was scanned on top of a calibration phantom, which was positioned at the level of the affected vertebra (Image Analysis, Columbia, Kentucky, United States). Using this phantom, the CT intensities of voxels representing bone tissue were converted into calcium equivalent values. Subsequently, isotropic material properties were assigned to each element based on the work by Keyak et al. (2005) [180]. For the upper and lower vertebrae, material behavior was assumed to be linear elastic. In contrast, non-linear elastic-plastic material behavior was assigned to the middle vertebra. More specifically, when the yield stress was reached, plasticity was induced and further defined by an initial ideal plastic phase, subsequent softening, and a final ideal plastic phase. Within the IVD, the NP and AF were modeled using hyperelastic Mooney-Rivlin material models. The AF was further modeled as a composite material with embedded rebar elements used to represent the collagen fiber reinforcement. The fibers were placed circumferentially at $\pm 20^{\circ}$ with the disc's local transverse plane and only acted in tension. Material properties were obtained from literature (NP: $\mathrm{C} 10=0.2 ; \mathrm{C} 01=0.045 ;$ bulk modulus $(\mathrm{K})=10^{6} \mathrm{MPa}$. AF ground substance: $\mathrm{C} 10=0.21 ; \mathrm{C} 01$ $=0.045 ; \mathrm{K}=1.2 \times 10^{6} \mathrm{MPa}$. Fibers: Young's modulus $(\mathrm{E})=650 \mathrm{MPa}$; Poisson's ratio $\left.(\mathrm{v})=0.33\right)$.

\subsubsection{FE simulations}

Both the experimental and physiological boundary conditions were, consecutively, applied to the model. The workflow for simulating the experimental setup has been described previously [177]. The specimen was loaded in axial compression until failure.

To apply the physiological boundary conditions, the global coordinate system of the FE model was transformed to align with the local reference frame of the L5 vertebra in the MSM. This way, L5, being the lower vertebra of the 3-segment spinal unit, could be constrained from translations and rotations in any direction. Subsequently, the FE model was transformed by visually aligning the FE and MSM L5 vertebrae. On the proximal end of the selected 3segment spinal unit, the L2/L3 joint resultant joint reactions forces and positions for all load cases obtained from the MSM were applied to the FE model. The loads and displacements were applied to a node of which the location matched the L2/L3 joint location of the MSM. This node was connected to the upper vertebra using rigid body elements type 2 (RBE2). The load cases were applied in a combined force- and displacement-controlled manner and in two consecutive steps. The first step resembled upright standing and in the second step the flexion, extension, and lateral bending maneuvers were simulated. 
Step I. Pre-conditioning simulating upright standing:

- A compressive force in the $\mathrm{y}$-direction equal to force in $\mathrm{y}$ at $\mathrm{t}=0$ calculated by the MSM was prescribed, representing upright standing.

- Displacements in the $\mathrm{x}$ - and z- directions were constrained.

Step II. Load application simulating the maneuvers:

- The forces in the y-direction calculated by the MSM, depending on the maneuvers, were prescribed.

- Translation in the forward (+x, flexion), backward (-x, extension), left (-z, left lateral bending), or right (+z, right lateral bending) direction only was prescribed, of which the magnitude corresponded to the magnitude of MSM's L2/L3 joint translation.

- Displacements in the irrelevant directions (i.e. z- direction for flexion/extension and xdirection for lateral bending) were constrained.

The FE simulations were performed with MSC Marc (MSC.MARC2013r1; MSC Software Corporation, Santa Ana, California, United States).

\subsubsection{Data analysis}

In order to compare the structural response of both models, displacements and forces predicted by the MSM were compared to those calculated by the FE model. Subsequently, fracture locations were qualitatively compared between the load cases. In order to compare loads that induced plasticity between axial compression and physiological movements, the load at initial plasticity in axial compression was considered. For this, we calculated the volume percentage of plastic elements (i.e. the summed volume of all elements with plasticity divided over the total volume of L4) at the end of the physiological movements and, subsequently, determined the axial reaction force at the increment in which the same volume percentage elements had turned plastic in the experimental loading configuration.

\subsection{Results}

\subsubsection{Comparison between displacements and forces predicted by the MSM and FE model}

Table 7.2 depicts the displacements and forces at the final stage of the applied movements (flexion, extension, lateral bending) predicted by the MSM and FE model. For flexion (45 $\left.{ }^{\circ}\right)$ and extension, the magnitude of the reaction forces in the $\mathrm{x}$ - direction was smaller in the $\mathrm{FE}$ 
Table 7.2: Comparison of the displacements and reaction forces at the L2/L3 joint at the end of the simulations of the MSM and FE model. For all movements, the force in the $y$ - direction and displacements in the $\mathrm{x}$ - and $\mathrm{z}$ - directions were prescribed. Therefore, the values predicted by the FE are very similar to the values predicted by the MSM (highlighted in italic). For comparing the responses of the FE and MSM, for flexion and extension the displacement in the $\mathrm{y}$ - direction and force in the $\mathrm{x}$ - direction should be considered and for lateral bending the displacement in $\mathrm{y}$ - direction and force in the z- direction are of most interest (highlighted in black). Numbers lower than 0.001 were considered to be 0 . Displacements are given in milimeters and forces in Newtons.

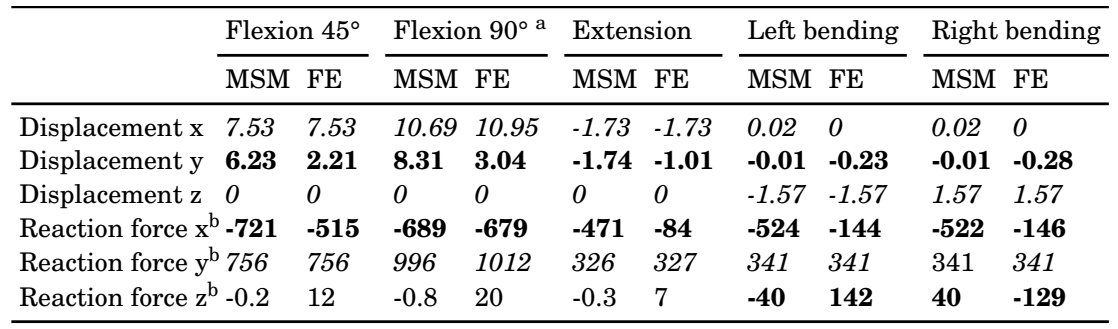

a This FE simulation did not converge to a point where the full MSM displacement in the $\mathrm{x}$ - direction was reached. For comparison with the MSM, the stage of the MSM in which a similar displacement in the $\mathrm{x}$ - direction was predicted is depicted.

b We corrected for differences in definitions obtained by the MSM and FE model, by flipping the sign of the reaction forces calculated by the FE model.

model than in the MSM model. Similarly, the displacements predicted in the y-direction were smaller in the FE compared to the MSM model. For the $90^{\circ}$ flexion task, the predicted reaction forces in the $\mathrm{x}$-direction were similar for both models, but the displacements were not.

Conversely, for lateral bending, the reaction forces in the z- direction were larger in the $\mathrm{FE}$ model than in the MSM model. In addition, the displacements in $\mathrm{y}$ - direction predicted by the FE model were larger than the displacements predicted by the MSM.

\subsubsection{Comparison between physiological movements and the experimental setup}

While in extension and lateral bending no plasticity was observed, a few elements, mainly on the surface of the lesion, became plastic in $45^{\circ}$ flexion (Fig. 7.3 . In $90^{\circ}$ flexion the region of plasticity had slightly increased when compared to the $45^{\circ}$ flexion movement (Fig. 7.3 .

The load to failure of the specimen in the experimental setup was $3741 \mathrm{~N}$. At this point of failure, plasticity was observed at the posterior part of the vertebral body including its posterior wall (Fig. 7.4 . 

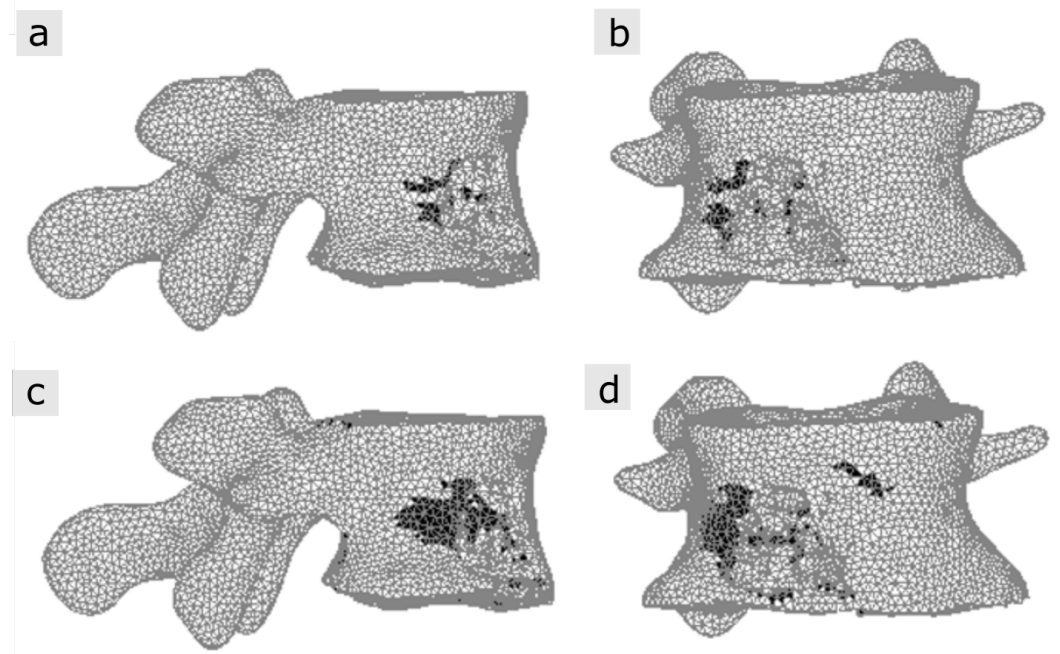

Figure 7.3: Plasticity (elements shown in black) induced in L4 by $45^{\circ}$ flexion (a-b) and $90^{\circ}$ flexion (c-d). Sagittal and frontal views are shown.
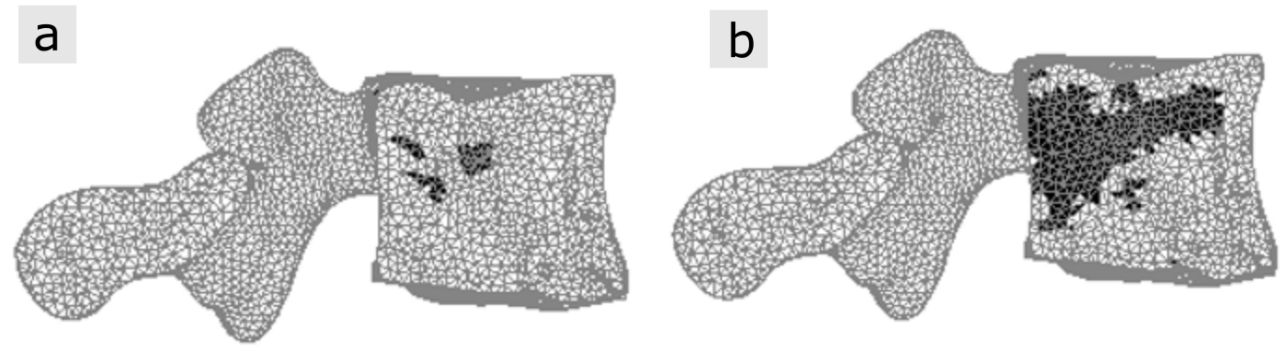

Figure 7.4: Plasticity (elements shown in black) induced in L4 by an axial compressive load at (a) initial plasticity and (b) structural failure. Cross-sections through the midsagittal plane are shown.

Of the physiological movements, only in flexion plasticity was observed. To compare the spinal loads under flexion and axial compression, we calculated the volume percentage of plastic elements at $45^{\circ}$ flexion and, subsequently, determined the axial reaction force at the increment in which the same volume percentage elements had turned plastic in the experimental loading configuration (hereinafter referred to as axial compression at initial plasticity) (Fig. 7.4). The load at initial plasticity in axial compression was $2267 \mathrm{~N}$.

Figure 7.5 illustrates the total joint reaction forces in the end stages of the different physiological loading conditions, as well as the axial reaction forces at initial plasticity and structural failure in the experimental loading configuration. The total joint reaction force 


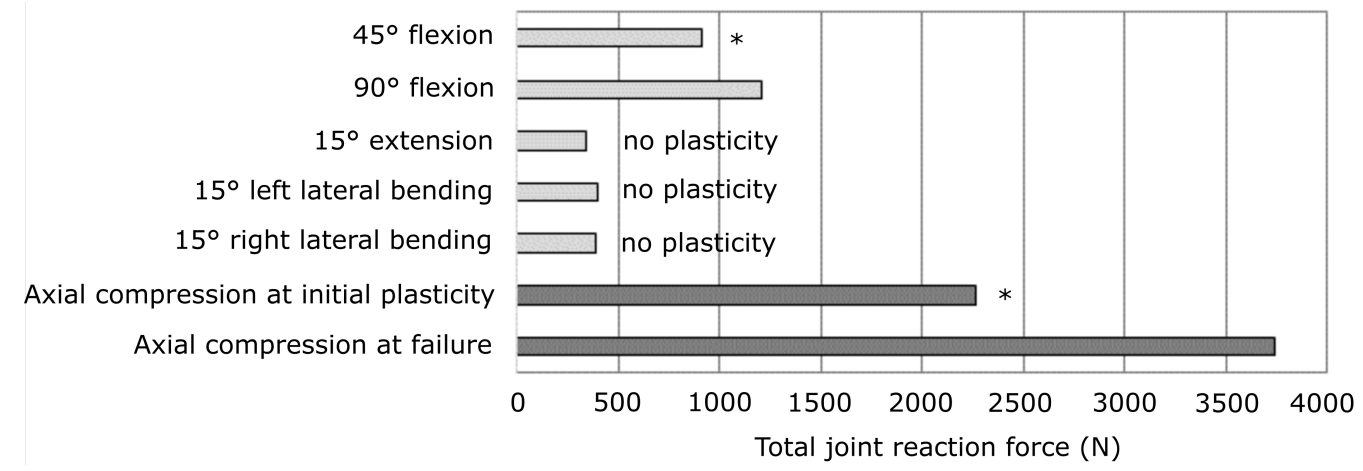

Figure 7.5: Total joint reaction forces calculated by the FE model for the end stages of the different physiological loading conditions (light grey bars) as well as the axial reaction forces in the experimental loading configuration (dark grey bars). For the experimental loading configuration, the axial reaction force at the increment at which the volume percentage of plastic elements was the same as the volume percentage of plastic elements in $45^{\circ}$ flexion (axial compression at initial plasticity) and the axial reaction force at structural failure are depicted. It is shown that the total joint reaction force in flexion was more than two times lower than the force needed to induce the same amount of plasticity under axial compression (bars indicated with *).

at $45^{\circ}$ flexion $(915 \mathrm{~N})$, in which some plasticity was observed, was considerably lower than the force required to induce a similar volume percentage of plastic elements in axial compression $(2267 \mathrm{~N})$. Furthermore, the force needed to induce failure in axial compression $(3741 \mathrm{~N})$ appeared to be approximately nine times higher than the total joint reaction forces induced by extension (338 N) and lateral bending (left: $396 \mathrm{~N}$; right: $393 \mathrm{~N}$ ).

\subsection{Discussion}

In this exploratory study physiological load cases predicted using musculoskeletal modeling were applied to an FE model of a real patient with spinal bone metastases who was treated within the prospective RACOST study, and were compared with a load case resembling an in vitro experimental setup. The goal of this study was to demonstrate that modeling physiological load cases can have a significant effect on failure predictions when compared to the simulation of a simple loading regime.

Of the physiological load cases, only flexion caused plasticity, although this did not directly lead to structural failure of the vertebral body. Although only a minor amount of plasticity was seen at $45^{\circ}$ of flexion, the total joint reaction force in flexion was more than two times lower than the force needed to induce the same amount of plasticity under axial compression. This may indicate that the vertebra under study was more prone to failure under flexion 
than compression. Also, the location in which plasticity occurred differed between the two loading configurations. Whereas in axial compression plasticity was mostly seen in the posterior part of the vertebral body, in flexion plasticity was mainly observed around the lesion located in the anterior part of the vertebral body. This emphasizes the importance of testing multiple physiological loading configurations when predicting the in vivo fracture risk.

The patient described in the current study sustained a pathological fracture of L4 during follow-up 11 months after stereotactic radiotherapy ( 1 x 20 Gy). When loading the L3-L5 specimen in axial compression, however, failure was not predicted to occur in L4, but in L3 (data not shown). For this reason, we assigned elastic rather than elastic-plastic material behavior to L3 and L5. It is unknown what movement or activity caused the major collapse in the patient's L4, which may have been different from axial compression. This, therefore, again implies that different loading configurations should be considered when predicting the in vivo fracture risk.

For flexion and extension the magnitude of the reaction forces were smaller in the FE model than in the MSM, whereas for lateral bending the reaction forces were larger in the $\mathrm{FE}$ model. When underestimating the forces while plasticity being absent, one should be careful with concluding that plasticity is not induced by that specific movement. The absence of plasticity could be caused by the underestimation of the applied forces. Conversely, when the FE model overestimated the forces predicted by the MSM while plasticity being absent, it could be safely concluded that that particular movement did not induce plasticity. Thus, it is highly unlikely that $15^{\circ}$ lateral bending would introduce plasticity in this patient.

This study has a considerable number of limitations and should be considered as an exploratory study to assess in vivo fracture risk. Firstly, we used a combined force-controlled and displacement-controlled simulation. Ideally, a purely force-controlled simulation should have been used, thereby prescribing only forces in the $\mathrm{x}-$, $\mathrm{y}$ - and $\mathrm{z}$ - directions. However, adopting this approach led to either numerical convergence problems or non-physiological failure patterns, movements, or reaction forces. An alternative approach would be to prescribe displacements in all directions. However, when performing purely displacement controlled simulations, similar problems were observed. These problems were likely due to a mismatch in dimensions of the MSM and FE model. The MSM was linearly scaled to the FE model based on body height, while differences in spinal curvature and vertebral morphology were ignored, resulting in geometrical differences between the MSM and FE model. Consequently, applying the displacements of the MSM L2/L3 joint to the FE model resulted in non-physiological deformations of the spine. Eventually, the resulting joint reaction forces and positions were directly applied to the FE model, which, due to the mismatch in spinal curvature, vertebral morphology, and joint location, may have induced 
differences in the applied overall joint loads.

Furthermore, the MSM explicitly modeled joint stiffness by using torsional springs, representing the stiffness of the discs, ligaments and facet joints. In contrast, the FE model only included the intervertebral discs and did not take into account stiffness due to spinal ligaments. A discrepancy in joint stiffness between the MSM and FE model may have contributed to the convergence problems. Moreover, we observed that the forces and displacements predicted by the MSM did not fully match the forces and displacements calculated by the FE model. This may also be due to the discrepancy in spinal dimensions and joint stiffness.

The movements simulated in the current study are not very demanding for the spine. Tasks like lifting an object, forward bending while carrying a weight, or walking downstairs will most likely cause higher loads in the spine. However, the MSM does not have the ability to simulating such movements yet. Hence, future MSM studies on vertebral loads should take these more demanding task in account to enable a more robust probing of vertebral strength.

\subsection{Conclusion}

In conclusion, this study demonstrated a first attempt to combine musculoskeletal and FE modeling in spinal bone metastases in a real patient case. Modeling physiological load cases instead of a simple loading regime significantly affected the failure predictions, indicating that for in vivo fracture prediction physiological load cases should be considered. Substantial improvements regarding the FE model, MSM, as well as combining MSMs and FE models are required in order to be able to predict plasticity under physiological loads.

\section{Acknowledgments}

We would like to thank Gill McColl for her help with the data management of the RACOST study, and Jasper Homminga and Bart Koopman for their help with developing the musculoskeletal model. 


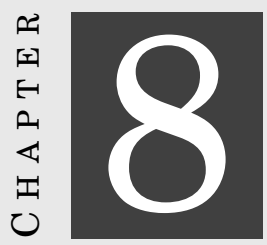

GENERAL DISCUSSION

This chapter provides a general discussion on the key findings of this dissertation and reflects on current limitations and future works. 


\subsection{Discussion}

The human spine undergoes substantial loads while maintaining essential functions and is prone to several diseases and complications. Understanding the nature of these loads can help to identify underlying conditions that lead to spinal pathologies as well as to improve existing treatment options. The current literature indicates that the spinal loads in healthy individuals were previously investigated for several activities of daily life, but that these measurements were limited to only certain thoracic and lumbar discs [1]. A detailed investigation of the load distribution throughout the spine can further our understanding of its in vivo functioning but is largely unavailable. The primary objective of this thesis work was to develop a validated-complete and coherent musculoskeletal model of the entire human spine for investigating the spinal loads. This chapter reflects on the key findings of this dissertation and provides future perspectives for further improvement and application of the developed model.

\subsubsection{Anatomical aspects}

In Chapters 2 \& 3, an anatomical dataset featuring musculoskeletal parameters needed for developing a complete and coherent musculoskeletal model of the entire human spine was obtained. This dataset, entitled as the Twente Spine Dataset, was measured by medical imaging and detailed dissection of one embalmed male cadaver (body height: $154 \mathrm{~cm}$, mass: $51 \mathrm{~kg}$ ). Muscles were divided into a number of muscle-tendon elements (actuators) for which the coordinates of attachment sites (origin, insertion, and via points) and architectural parameters were reported. The architectural muscle parameters include fiber length, tendon length, sarcomere length, optimal fiber length, pennation angle, mass, and physiological cross-sectional area (PCSA) which were previously shown to affect musculoskeletal predictions to a large extent [43].

Borst et al. (2011) reported attachment locations and architectural parameters of all neck muscles from one cadaver [38]. Other researchers measured only some of the muscle parameters from several cadavers and illustrated muscle attachment sites in the lumbar and cervical regions [42, 52-58]. Differently from these earlier studies [42, 52-58], the Twente Spine Dataset was measured from a single cadaver. Thus, this dataset enables developing a coherent musculoskeletal model for the entire human spine and eliminates uncertainties intrinsic in piecing anatomical data from different spines [60, 76]. Furthermore, this dataset provides new data for several muscle groups such as serratus posterior inferior, rotatores, multifidus, levatores costarum, spinalis, semispinalis, subcostales, transversus thoracis, and intercostales muscles. As such, the Twente Spine Dataset comprises the first complete and consistent atlas for modeling the human spine. Furthermore, muscle parameters measured 
in the Twente Spine Dataset were seen to fit within the range of data presented in previous anatomical reports. For measured muscles, relatively shorter optimal fiber lengths were calculated compared to the other studies, and this was primarily linked to the differences between the heights of the cadavers dissected $(154 \mathrm{~cm}$ in this study and around $170 \mathrm{~cm}$ in other studies). The mean sarcomere lengths of some muscles located around the neck were higher than those given in [38, 52].

Several muscle groups-presented in the Twente Spine Dataset-had multiple attachments at different bones, and anatomical variabilities on muscle attachment sites were found. For example, scalenus anterior muscle had origin attachments at the cervical fifth (C5) to seventh vertebrae (C7) whereas standard anatomy books such as Gray et al. (1973) [100] illustrates the origin attachments of this muscle at the cervical third (C3) to sixth vertebrae (C6). These differences were previously described in Gray's book [100] and can be attributed to the variations in the anatomy of the specimens and the differences in the measurement techniques. It can be of a great value to know if these variabilities would lead to significant differences in muscle coordination strategies and can be linked to existing spinal pathologies. Furthermore, such variabilities in muscle attachment sites make developing subject-specific models from anatomical atlases somewhat challenging. In the recent years, some authors developed novel methods to extract moment arms and cross-sectional areas of major trunk muscles from medical images and implement those to create subject-specific models from generic atlases [31, 33, 41]. Although such protocols will work great for large muscle groups, they are bound to technical difficulties with measuring attachment sites of the small and deeper muscles accurately. Existing imaging techniques have great potential to create fully customized, subject-specific models but should be improved to tackle these limiting factors [50, 136].

Investigation of the transversospinal muscle anatomy (including levatores costarum and spinalis thoracis muscles) revealed apparent differences in their morphologies compared to other muscle groups which had tendon-muscle fiber-tendon compositions (Chapter 3). Firstly, most elements of the multifidus, spinalis thoracis, levatores costarum, and rotatores had tendons running from origin to insertion attachments, and the tendons were mixed with muscle tissues. In these muscles, muscle fibers either spanned the full length of the tendon or partially did. Secondly, these muscle elements had very small PCSAs. Considering that active force contributions would be small in these elements, a different approach rather than employing Hill-based muscle models can be exercised, which should focus on the passive behavior (for example, modeling them as ligaments). Their morphologies signal that they might essentially be responsible for stabilizing our joints beyond physiological movements [71, 102, 103]. More research is needed to identify other possible functions of these muscles and to model their actions. 


\subsubsection{Estimation of spinal loads}

In vivo assessment of the internal loads in the spine has been previously done in a number of studies where intervertebral disc pressures [3]-9] or instrumented implant loads [10, 11] were measured. When measuring intradiscal pressures, typically a pressure transducer is placed inside the nucleus pulposus region of the discs, and subjects are instructed to maintain certain trunk postures. Differently, resultant forces and moments are measured through instrumented implants (used to replace lumbar vertebral bodies) during quasistatic or dynamic activities. As both measurement techniques are invasive by nature, there have been limitations as to measured spinal levels and activities. Moreover, shear forces at the intervertebral discs or muscle forces can not be estimated from previously measured intradiscal pressures. Unfortunately, these limitations hinder extending our understanding of the interplay between spinal loads and locomotion. In short, there is a need for a detailed assessment of the internal load distribution throughout the spinal column.

Computational models representing the musculoskeletal system enable a non-invasive and comprehensive assessment of the spinal loads. In the last decade, there have been a large number of models developed to investigate the internal loading within the cervical spine [35, 36], lumbar spine [26-32], thoracolumbar spine [33, 34], and cervico-thoracolumbar spine [37]. Historically, these models were created for representing generic or subject-specific musculoskeletal systems with increasing complexity. Generic models of the spine generally incorporate averaged muscle data measured from several individuals but skeletal data from one single person. In such representations, scaling between the skeletal geometries of the spines and the muscle parameters should be considered to ensure a realistic anatomy. However, a physiologically-sound scaling protocol has not been previously designed nor applied to existing generic datasets or spine models [26-28, 37, 40]. Some of these models have been validated against experimentally measured intervertebral disc pressures, muscle activations, or maximal isometric joint moments. Furthermore, the majority of these models used anatomical illustrations to specify muscle attachment sites and did not include deeper spinal muscles. Some authors presented novel techniques towards implementing subject-specific muscle paths and architectural parameters from medical images [31, 33, 41]. However, these methods are subject to technical limitations and need further improvements. For example, lines-of-action of deeper and smaller muscles might not be reliably measured, and some muscle parameters such as PCSA, sarcomere length, and optimal fiber length can not be accurately estimated from two-dimensional images [42, 44]. In Chapter 4 a musculoskeletal model of the entire human spine (the Twente Spine Model) was created based on the dataset presented in Chapters 2 \& 3 This model includes cervical, thoracic, and lumbar regions, a flexible ribcage, and a complete muscular anatomy which included several deeper muscles in the trunk and ribcage. Attachment sites and architectural parameters of all the muscles were implemented precisely as measured in 
the dataset. Since a coherent set of musculoskeletal data was used, this model essentially represents a subject-specific musculoskeletal system. It has been previously postulated that subject-specific models (compared to generic models) include better representations of the musculoskeletal anatomy [38, 39]. While it is true that the Twente Spine Model is a highly subject-specific model and may not represent average subject populations, it comprises a realistic musculoskeletal anatomy and should, thus, yield physiological prediction trends which are valid for healthy spines.

In Chapter 4 basic physiological movements such as flexion, extension, lateral bending, and axial rotation of the spine from a standing upright position were simulated by using the Twente Spine Model. The joint and muscle forces were computed by using an inverse static approach and optimization criterion which minimized muscle fatigue. Intervertebral disc compressive forces in the trunk and neck regions were presented as functions of the trunk and neck inclinations, respectively. Results indicated these forces were very dependent on the movement direction and magnitude and disc location. Among all the maneuvers, trunk flexion generally induced the largest compressive loads at the thoracic ( $\sim 150 \% \mathrm{BW}$ at the $\mathrm{T} 11 / \mathrm{T} 12 \mathrm{disc})$ and lumbar ( $250 \% \mathrm{BW}$ at the L5/S1 disc) intervertebral discs at the final positions. For the same trunk angles ( $30^{\circ}$ flexion, lateral bending, and axial rotation), axial rotation of the trunk caused the largest loads at the thoracic discs (as high as $\sim 117 \% \mathrm{BW}$ at the T11/T12 disc), whereas forward flexion induced the largest loads at the lumbar discs ( $\sim 165 \%$ BW at the L5/S1 disc). Furthermore, disc compressions generally increased caudally and with increasing trunk motion but in extension at which they dropped at the lumbar and lower thoracic levels. During all trunk maneuvers, the compression force at the T11/T12 disc was generally higher compared to the $\mathrm{T} 12 / \mathrm{L} 1$ disc. The compressive forces at the cervical discs were significantly lower than those of the thoracolumbar discs during executions of the neck tasks. Cervical disc forces generally increased during neck flexion, lateral bending, and axial rotation but gradually decreased in extension. Lateral bending caused relatively higher loads at the lower cervical discs (43\% BW at the $\mathrm{C} 7 / \mathrm{T} 1$ level) whereas axial rotation induced the largest loads at the upper discs (as high as 56\% BW at the $\mathrm{C} 1 / \mathrm{C} 2$ level) during final stages of the maneuver.

In this thesis, spinal loads predicted by using the Twente Spine Model were compared to previous in vivo measurements from several subjects [3- $[$, 10]. For this, quasi-static trunk tasks as measured in these studies were simulated, and resulting intradiscal pressures at some thoracic and lumbar discs and normalized resultant loads were calculated. In general, good agreements with the in vivo measurements were found for the intradiscal pressures at the L4/L5 and L3/L4 levels. The compression forces at the L3/L4 disc during forward flexion and side bending were slightly underpredicted, but the increase trends compared to the upright position were predicted well in the model. Calculated disc pressures at the T6-T8 and T9-T11 levels were generally within the reported range. In addition, the changes compared 
to the upright position were captured well except for the lower thoracic discs during extension. The relative pressures between the middle and lower thoracic discs were in accordance with the in vivo data except for lateral bending. Furthermore, calculated disc pressures for the upright position were in excellent agreement with the experimental data. The pressures dropped caudally from $0.85 \mathrm{MPa}$ at T6-T8 and $0.71 \mathrm{MPa}$ at T9-T11 to $0.51 \mathrm{MPa}$ at L3/L4 but later increased to $0.55 \mathrm{MPa}$ at L4/L5 disc [1]. Lastly, the model' estimations of the normalized resultant loads (as the percentage of the load at upright standing) were within the reported range for the L1 vertebrae. However, in extension, the model overpredicted the normalized loads. It was not possible to validate predicted compression forces at the cervical discs since no in vivo intradiscal pressure data on healthy discs were available. Snijders et al. (1991) [132] found in his cervical model that disc forces increased during flexion, decreased in extension, increased in lateral bending, and increased during axial rotation after around $35^{\circ}$ neck inclination. Cervical disc compression forces calculated in the Twente Spine Model were in good agreement with these findings. Moreover, compressive forces at the standing upright position (expressed as percentages of the total body weight) were comparable to those reported in a cervico-thoraco-lumbar spine model [37]. The variations in the measured data and the differences with our findings can be explained by several subject-specific and modeling features. These features are multifactorial and include differences in movement coordination [85, 127, 155] and spinal curvature [86, 136], and the idealization of the arms as lumped masses, simplification of the mechanical function of the spinal ligaments [135, 139] and the lack of facet joints [12,33] in the model. Although some discrepancies, generally good agreements with the previously measured in vivo spinal loads were found. This showed a first validation of the Twente Spine Model for an attempt to predict the compression forces at the intervertebral discs during basic activities of daily life. It should be emphasized that relatively light activities were simulated with the model for the validation process. A further validation in terms of muscle forces and joint moments during more demanding tasks is necessary. This will contribute to the credibility of the model for studying occupational tasks such as manual materials handling and should be the study topic for a future research.

Disc compressive forces (in \% BW) predicted in the Twente Spine Model for the upright position were systematically higher than the predictions of previous models for all spinal levels [28, 34, 37, 40, 130], but were within measured in vivo data range. Predicted compressive forces were closest to those presented from a generic, thoracolumbar, spine model which implemented a single-lumped rigid body representation of the neck and overpredicted thoracic intradiscal pressures [34]. Furthermore, anterior-posterior shear forces at the standing position were also generally higher than those reported in earlier models. Earlier models found shear forces ranging from $\sim 4 \%$ to $\sim 10 \% \mathrm{BW}$ at the L1/L2 disc, compared to this study (6\% BW) and the experimental data $\% 10 \mathrm{BW}$ [10]. This showed that the shear force predicted at the L1/L2 level was in agreement with the in vivo data and previous models. Unfortunately, comparing shear forces calculated at the other spinal levels was not 
possible since in vivo data was not available. The differences between the findings of the earlier works and the cadaver-based model can possibly be explained due to differences in represented muscular and skeletal anatomies or modeling assumptions.

\subsubsection{Sensitivity of musculoskeletal predictions}

In the past, several researchers investigated the sensitivity of their model to certain musculoskeletal parameters such as vertebral geometry, ligament properties, spinal rhythms, intervertebral disc joint locations or modeling assumptions [40, 48, 85, 138]. They discussed that the prediction of the intervertebral disc and muscle forces were significantly affected by the studied parameters. In Chapter 5 , the sensitivity of muscle and disc force (compressive and shear) computations against potential errors in modeling muscle attachment sites (origin, insertion, and via points) were investigated. Biomechanical simulations showed that small errors in modeling the muscle attachment sites affected predicted disc forces inconsiderably to significantly. The majority of the muscle groups had negligible effects (typically less than 5\% change) in the disc forces, while some muscles such as quadratus lumborum, changed the compressive and shear forces as high as 17\% and 353\% (T6/T7 disc), respectively. In addition, disc shear forces were generally more sensitive (in percentage) to changes in the muscle attachment sites than disc compressive forces. This was primarily due to the fact that nominal compressive forces were much higher compared to nominal shear forces.

Muscle forces, as well as the recruitment patterns, were also sensitive to perturbations in attachment locations, particularly of certain muscles in the ribcage and lumbar region. These muscles were quadratus lumborum, intercostales interni, longissimus thoracis, and intercostales externi which had attachments at several bones. They contribute to joint moments at the costo-sternal, costo-vertebral, costo-transverse or lumbar intervertebral articulations. Presented findings show that altered moment arms (increased or decreased) made these muscles less favorable and required other muscles to maintain the joint moments [51, 144]. Higher sensitivity indexes computed for these muscles were associated with the changes in disc forces. This shows the dependency of computed spinal loads on muscle activation patterns and, thus, to their attachment locations. Nussbaum et al. (1995) found that spinal forces were very sensitive to moderate changes in the lines-of-action of oblique abdominal muscles but were less sensitive for vertically oriented muscles [140]. Chapter 5 presented that disc forces were sensitive to attachments of the external and internal abdominals and some vertically oriented muscles. However, the simulations did not indicate oblique muscles as yielding the highest sensitivity nor the vertical muscles being the least influential. These differences found between the studies can be explained due to incorporating different spinal anatomies and modeling assumptions or the representation of the spinal anatomy. 
When developing a generic model, it is a general practice to use anatomical illustrations for defining muscle lines-of-action. The results presented in this thesis indicate that this practice only slightly affects model predictions for most muscles, while considerable errors on model predictions can result for certain muscles. It is, thus, advised to use anatomical datasets where muscle attachment locations were quantified precisely. Furthermore, particular attention should be given when morphing muscle attachments for developing personalized models of the spine, especially for the muscles highlighted in this thesis.

\subsubsection{Model validation aspects}

It is crucial to gain confidence with model predictions before use in basic or applied clinical research. Models of complex biological systems should be critically evaluated to characterize any forms of uncertainty and error in simulation outputs [147]. This can be achieved by comparing outputs of interest with experimentally measured data. Because the musculoskeletal information that can be accessed non-invasively is limited, qualitative and indirect comparisons are often used. Hence, model validation has been inherently a challenging task for spinal musculoskeletal models. Surface electromyography is an indirect but non-invasive technique that can help in the validation process. For example, predicted muscle activities or forces of spinal muscles can be compared with electromyography recordings [30, 33, 109].

In Chapter 6 trunk movements and electromyographic activities of the major trunk and abdominal muscles were measured simultaneously in fourteen healthy subjects during isometric and dynamic trunk exertions. Several muscle groups played roles during basic trunk movements (flexion, extension, lateral bending, and axial rotation of the spine) and, consistent muscle activation patterns (among three subject groups) were found for most static and dynamics tasks. For example, abdominal muscles had higher activations towards the maximum ranges of movement in dynamic flexion. Furthermore, lumbar erector spinae ipsilaterally and obliquus externus abdominis and multifidus muscles bilaterally contributed to lateral bending. Despite these similarities between the subject groups, some gender and body height related differences in activation patterns were found. For example, the activity of obliquus externus abdominis muscle during isometric flexion did not change much compared to the upright position in the short male and tall male groups, but it slightly decreased in the short female group. Therefore, presented discrepancies should be taken into consideration when validating predicted muscle activations. A further study on larger population groups is necessary to determine the significance of these findings. Measured muscle activation trends of the back and abdominal muscles were mostly in line with previous studies [14, 156, 157, 162-164]. Measured spinal kinematics can be isometrically or dynamically simulated by using the data presented in this thesis, and muscle activation 
patterns can be compared to justify predictions of internal loading.

\subsubsection{Clinical application}

An accurate prediction of the fracture risk in a metastasized spine is essential for planning effective treatment methods for improving the quality of life of the patient. In Chapter 7 . musculoskeletal and finite element models were pipelined to study a patient case. This patient had a metastatic lesion at the fourth lumbar vertebra (L4), which eventually led to a pathological fracture. A finite element model of the L3-L5 spinal unit-including intervertebral discs-was developed and virtually tested under two different loading schemes. Namely, an axial compression force as typically applied in conventional in vitro tests or physiological loading conditions predicted using musculoskeletal modeling were used. The Twente Spine Model presented in Chapter 4 was scaled to match with the patient's body height and mass, and intervertebral disc forces were computed for basic trunk movements. Computer simulations showed that only flexion movement caused plasticity at the L4 vertebra, and that the disc force during $45^{\circ}$ flexed position was significantly lower than the axial force which resulted in the same plasticity level. This implied that the metastasized vertebra was more likely to fail under flexion loads than under only a compression load. This chapter provided a workflow which pipelined finite element and musculoskeletal models to study a clinical problem and suggests that physiological loading conditions should be considered when assessing vertebral fracture risks.

\subsubsection{Limitations and future work}

The findings discussed in this dissertation were affected by several limitations which are primarily related to the musculoskeletal model (Chapter 4) and anatomical dataset (Chapter 2 \& Chapter 3). Some major limiting factors will be briefly summarized here, and recommendations regarding for future works will be addressed. Firstly, masses and centers-of-mass of the body segments were defined by using previously reported anthropometrical ratios [106-108]. These parameters are likely to affect musculoskeletal predictions and can be extracted from the CT and MRI images reported with the dataset. Secondly, the developed model did not incorporate spinal ligaments but simplified their rotational stiffnesses at the intervertebral and ribcage joints. The missing ligaments or simplification of their mechanical function would alter muscle forces since passive ligaments contribute to force equilibriums at the crossed joints [135, 139]. Including the spinal ligaments in the model per se is not a difficult task, but requires relying on literature data. The elasticity of the spinal ligaments is affected by several factors such as age, mobility, and spinal level and changes considerably across previous studies [181, [182]. Thus, model calibration for optimizing ligament properties becomes critical for reliable assessment of the joint and soft tissue loads. With future advancements in the imaging modalities, it might be possible to 
implement ligament attachment sites and mechanical parameters from provided medical images. Thirdly, facet joints were not explicitly modeled in the Twente Spine Model. However, their contributions to rotational stiffnesses were accounted for in the disc stiffness coefficients. It was previously discussed that the facet joints will decrease the forces transferred through the anterior column during extension [12, 33]. Implementation of the facet joints will provide a more realistic characterization of the spinal loads for movements which involve large contact forces at these articulations. Fourthly, force-length and force-velocity relationships modulating muscle force production were not included. Although fiber lengths and tendon lengths were available in the dataset, tendon slack lengths require further calibrations. For example, we can calibrate the tendon lengths such that the muscles have their optimal fiber lengths at certain body postures. When we know those body postures corresponding to the optimal fiber lengths, we can set the joint positions in the model and adjust the tendon length for each muscle. Furthermore, a passive muscle force is produced when the muscle is stretched beyond its optimal fiber length. Incorporating these features will enable to assess active and passive muscle force contributions and should be considered in next generations of the model. Fifthly, the representation of the shoulder-arm complex can be improved. External forces and moments were applied at the third thoracic vertebrae to model the loads due to these extremities. Three-dimensional geometries of these segments are available in the dataset. These body segments can be included and the movements at the shoulder-arm joints can be simulated in the model. Sixthly, spherical joints were used for modeling the intervertebral disc articulations. Translational movements can also be modeled in the future [133, 134, 146]. This would allow a more realistic simulation of the movement at these joints and should be considered when studying applications where small changes in muscles' moment arms could affect the predictions much. Seventhly, when available, more realistic movements can be simulated at the costo-sternal and compound costo-vertebral and costo-transverse joints [183, 184]. In addition, it was found during dissection studies that the cadaver had slight scoliosis around the cervical region and that the fifth lumbar vertebra was fused to the sacrum. Thus, the architecture of the muscles around these regions might be affected by these abnormalities [78, 92]. Furthermore, morphological muscle parameters measured in vitro might be different from in vivo.

Subsequent chapters had also limitations. In Chapter 5, an optimization criterion minimizing the muscle fatigue was adapted to calculate the joint and muscle forces. Using a different performance criterion might affect the findings. In addition, the limited number of participants for EMG measurements makes it difficult to support the findings associated with the differences in gender and body height (Chapter 6). Furthermore, some attention should be given when interpreting the normalized muscle activities and kinematics for the dynamic movements in Chapter 7 since muscle cross talks and soft tissue artifacts might be present. Using needle EMG instead of surface EMG will help to eliminate the muscle cross talks to some extent but is also subject to ethical considerations. Lastly, the 
Twente Spine Model was linearly scaled to match the body height and mass of the patient in Chapter 7 A more realistic, subject-specific model might be developed by also considering the spinal curvature, muscle morphology, vertebral morphology, gender, age [41, 136].

\subsubsection{Conclusion}

The main contribution of this dissertation is the development of a complete and coherent musculoskeletal model of the entire human spine. Compared to existing models, this cadaverbased model includes the most comprehensive and realistic musculoskeletal anatomy of the spine and enables a thorough investigation of the spinal joint and muscle forces. Future work should be realized to improve the validity of the developed model for studying basic and clinical research questions. 



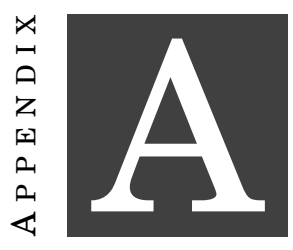

APPENDIX A

\section{A.1 Registration of the bones}

Registration of the STL files of the bones with the point clouds (highlighted as red dots) is illustrated in Fig. A.1. A mean error of $0.78 \mathrm{~mm}$ was found for all the bones. STL geometry files (defined in the same reference frame as for the muscle attachments) are available through https://www. utwente.nl/en/et/bw/research/projects/twentespinemodel.

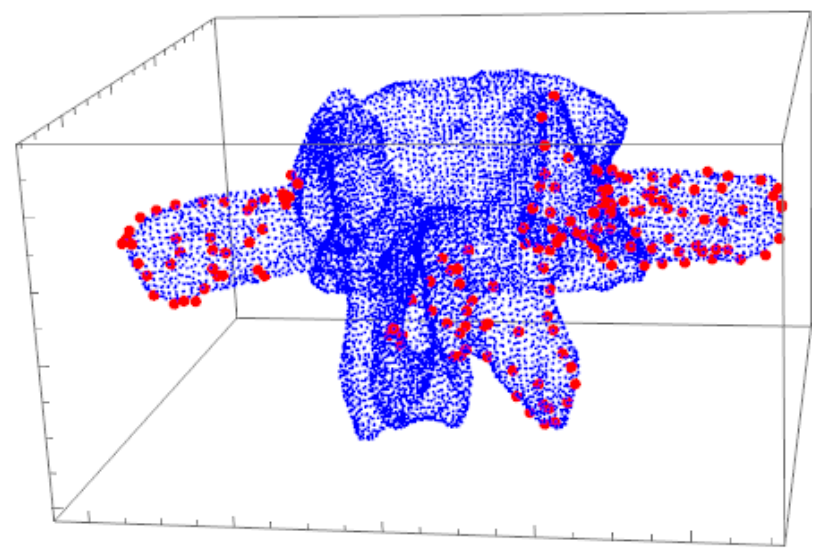

Figure A.1: Illustration of the registration of the bones with the point clouds. The geometry of L2 was plotted together with its point cloud. 


\section{A.2 Mathematical descriptions of wrapping surfaces}

Estimation of wrapping surfaces is illustrated in Fig. A.2 In this figure, red dots represent the point cloud measured over the bone surfaces of the femur and the pelvis. In this case, a cylinder was estimated for the psoas major muscle.

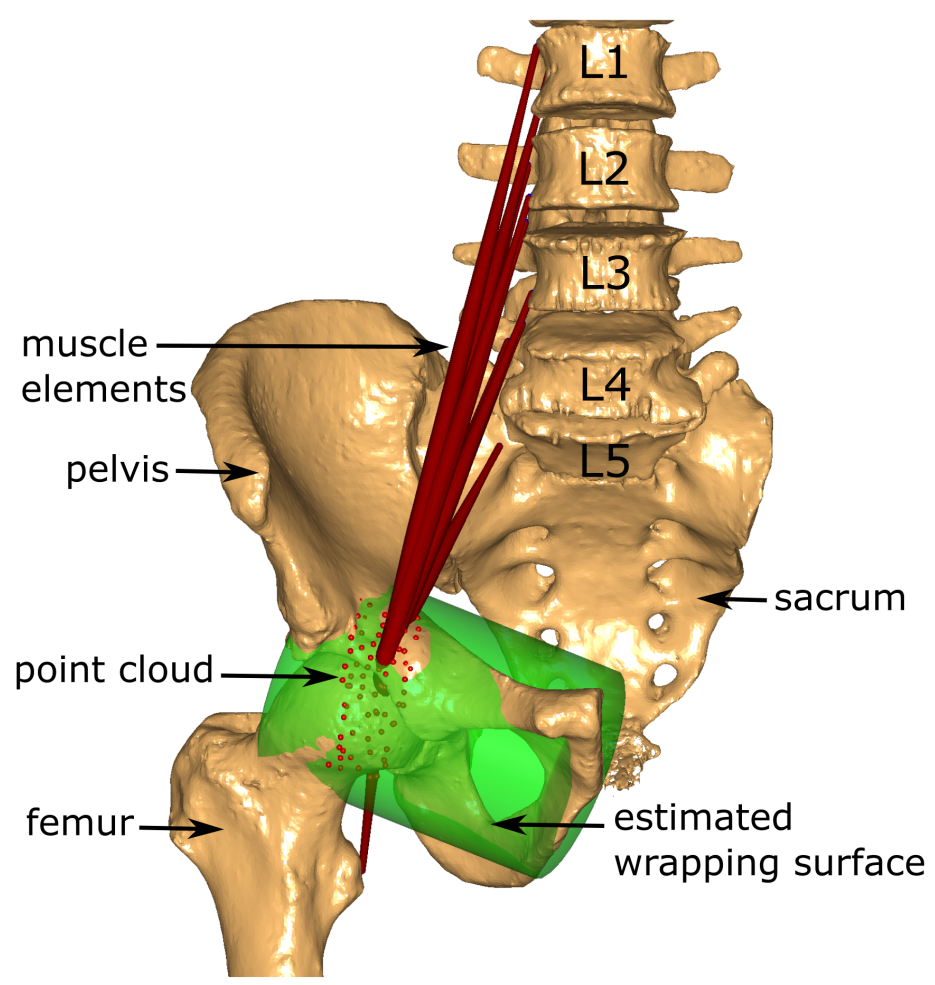

Figure A.2: Illustration of the wrapping surface for the psoas major muscle. 


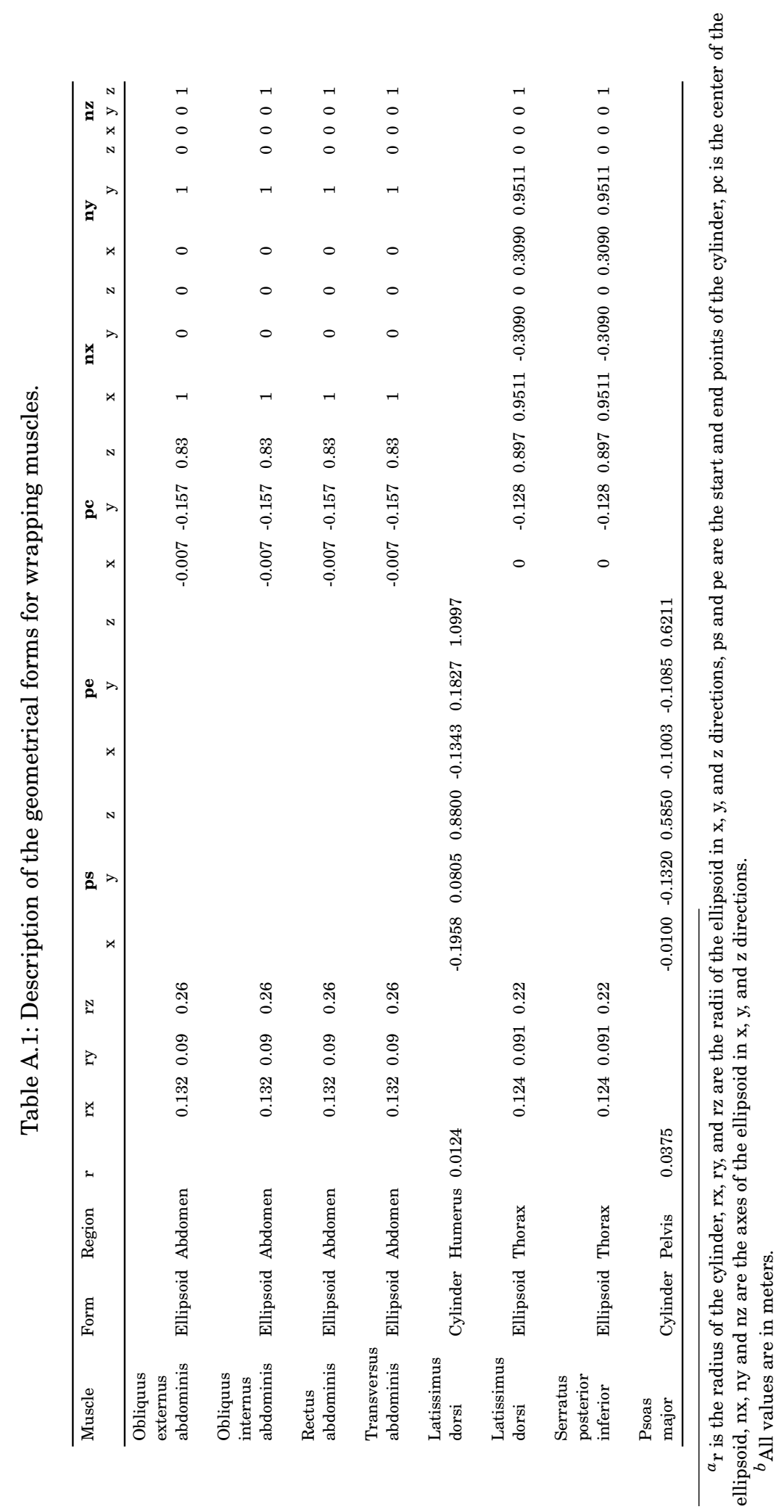




\section{A.3 Coordinates of the via attachments for muscle elements with a curved line-of-action.}

Table A.2: Coordinates of via attachments with respect to the global reference frame.

\begin{tabular}{|c|c|c|c|c|c|c|}
\hline \multirow[t]{2}{*}{ Muscle } & \multirow[t]{2}{*}{$\#$} & \multirow[t]{2}{*}{ Via } & \multirow[t]{2}{*}{ Bone } & \multicolumn{3}{|c|}{ Position (m) } \\
\hline & & & & $\mathrm{x}$ & $\mathrm{y}$ & $\mathrm{z}$ \\
\hline Iliocostalis lumborum & 1 & 1 & L1 & -0.0747 & -0.0593 & 0.8211 \\
\hline Iliocostalis lumborum & 1 & 2 & $\mathrm{~L} 2$ & -0.0695 & -0.0647 & 0.7875 \\
\hline Iliocostalis lumborum & 1 & 3 & L3 & -0.0652 & -0.0696 & 0.7589 \\
\hline Iliocostalis lumborum & 1 & 4 & $\mathrm{~L} 4$ & -0.0620 & -0.0732 & 0.7387 \\
\hline Iliocostalis lumborum & 1 & 5 & L5 & -0.0594 & -0.0770 & 0.7235 \\
\hline Iliocostalis lumborum & 2 & 1 & $\mathrm{R} 11$ & -0.0718 & -0.0546 & 0.8499 \\
\hline Iliocostalis lumborum & 2 & 2 & $\mathrm{~T} 12$ & -0.0704 & -0.0581 & 0.8383 \\
\hline Iliocostalis lumborum & 2 & 3 & $\mathrm{~L} 1$ & -0.0682 & -0.0616 & 0.8211 \\
\hline Iliocostalis lumborum & 2 & 4 & L2 & -0.0638 & -0.0675 & 0.7875 \\
\hline Iliocostalis lumborum & 2 & 5 & L3 & -0.0602 & -0.0717 & 0.7589 \\
\hline Iliocostalis lumborum & 2 & 6 & $\mathrm{~L} 4$ & -0.0575 & -0.0755 & 0.7387 \\
\hline Iliocostalis lumborum & 2 & 7 & L5 & -0.0555 & -0.0770 & 0.7235 \\
\hline Iliocostalis lumborum & 3 & 1 & $\mathrm{R} 10$ & -0.0707 & -0.0496 & 0.8911 \\
\hline Iliocostalis lumborum & 3 & 2 & $\mathrm{R} 11$ & -0.0660 & -0.0546 & 0.8499 \\
\hline Iliocostalis lumborum & 3 & 3 & $\mathrm{~T} 12$ & -0.0647 & -0.0591 & 0.8383 \\
\hline Iliocostalis lumborum & 3 & 4 & $\mathrm{~L} 1$ & -0.0627 & -0.0636 & 0.8211 \\
\hline Iliocostalis lumborum & 3 & 5 & $\mathrm{~L} 2$ & -0.0590 & -0.0700 & 0.7875 \\
\hline Iliocostalis lumborum & 3 & 6 & L3 & -0.0557 & -0.0737 & 0.7589 \\
\hline Iliocostalis lumborum & 3 & 7 & $\mathrm{~L} 4$ & -0.0534 & -0.0776 & 0.7387 \\
\hline Iliocostalis lumborum & 3 & 8 & L5 & -0.0517 & -0.0770 & 0.7235 \\
\hline Iliocostalis lumborum & 4 & 1 & R9 & -0.0755 & -0.0453 & 0.9286 \\
\hline Iliocostalis lumborum & 4 & 2 & $\mathrm{R} 10$ & -0.0707 & -0.0497 & 0.8911 \\
\hline Iliocostalis lumborum & 4 & 3 & $\mathrm{R} 11$ & -0.0657 & -0.0535 & 0.8530 \\
\hline Iliocostalis lumborum & 4 & 4 & $\mathrm{~T} 12$ & -0.0637 & -0.0587 & 0.8383 \\
\hline Iliocostalis lumborum & 4 & 5 & L1 & -0.0616 & -0.0640 & 0.8211 \\
\hline Iliocostalis lumborum & 4 & 6 & $\mathrm{~L} 2$ & -0.0573 & -0.0709 & 0.7875 \\
\hline Iliocostalis lumborum & 4 & 7 & L3 & -0.0536 & -0.0747 & 0.7589 \\
\hline Iliocostalis lumborum & 4 & 8 & $\mathrm{~L} 4$ & -0.0509 & -0.0790 & 0.7387 \\
\hline Iliocostalis lumborum & 4 & 9 & L5 & -0.0490 & -0.0770 & 0.7235 \\
\hline Iliocostalis lumborum & 5 & 1 & $\mathrm{R} 8$ & -0.0777 & -0.0494 & 0.9622 \\
\hline Iliocostalis lumborum & 5 & 2 & $\mathrm{R} 9$ & -0.0736 & -0.0450 & 0.9300 \\
\hline Iliocostalis lumborum & 5 & 3 & $\mathrm{R} 10$ & -0.0698 & -0.0460 & 0.9000 \\
\hline Iliocostalis lumborum & 5 & 4 & $\mathrm{R} 11$ & -0.0645 & -0.0505 & 0.8587 \\
\hline Iliocostalis lumborum & 5 & 5 & $\mathrm{~T} 12$ & -0.0620 & -0.0576 & 0.8383 \\
\hline Iliocostalis lumborum & 5 & 6 & $\mathrm{~L} 1$ & -0.0597 & -0.0647 & 0.8211 \\
\hline Iliocostalis lumborum & 5 & 7 & L2 & -0.0554 & -0.0719 & 0.7875 \\
\hline Iliocostalis lumborum & 5 & 8 & L3 & -0.0517 & -0.0755 & 0.7589 \\
\hline Iliocostalis lumborum & 5 & 9 & $\mathrm{~L} 4$ & -0.0492 & -0.0800 & 0.7387 \\
\hline Iliocostalis lumborum & 5 & 10 & L5 & -0.0472 & -0.0770 & 0.7235 \\
\hline Iliocostalis lumborum & 6 & 1 & $\mathrm{R} 7$ & -0.0685 & -0.0537 & 0.9881 \\
\hline Iliocostalis lumborum & 6 & 2 & $\mathrm{R} 8$ & -0.0662 & -0.0477 & 0.9626 \\
\hline Iliocostalis lumborum & 6 & 3 & R9 & -0.0633 & -0.0449 & 0.9313 \\
\hline Iliocostalis lumborum & 6 & 4 & $\mathrm{R} 10$ & -0.0610 & -0.0467 & 0.9050 \\
\hline Iliocostalis lumborum & 6 & 5 & $\mathrm{R} 11$ & -0.0579 & -0.0513 & 0.8700 \\
\hline Iliocostalis lumborum & 6 & 6 & $\mathrm{~T} 12$ & -0.0550 & -0.0592 & 0.8383 \\
\hline Iliocostalis lumborum & 6 & 7 & $\mathrm{~L} 1$ & -0.0535 & -0.0672 & 0.8211 \\
\hline Iliocostalis lumborum & 6 & 8 & L2 & -0.0505 & -0.0743 & 0.7875 \\
\hline Iliocostalis lumborum & 6 & 9 & L3 & -0.0479 & -0.0771 & 0.7589 \\
\hline Iliocostalis lumborum & 6 & 10 & $\mathrm{~L} 4$ & -0.0461 & -0.0815 & 0.7387 \\
\hline Iliocostalis lumborum & 6 & 11 & L5 & -0.0447 & -0.0768 & 0.7235 \\
\hline Longissimus thoracis & 1 & 1 & $\mathrm{~T} 4$ & -0.0415 & -0.0673 & 1.0310 \\
\hline Longissimus thoracis & 1 & 2 & T5 & -0.0392 & -0.0580 & 1.0110 \\
\hline Longissimus thoracis & 1 & 3 & $\mathrm{~T} 6$ & -0.0362 & -0.0510 & 0.9855 \\
\hline
\end{tabular}

Continued on next page 


\begin{tabular}{|c|c|c|c|c|c|c|}
\hline \multirow[t]{2}{*}{ Muscle } & \multirow[t]{2}{*}{ \# } & \multirow[t]{2}{*}{ Via } & \multirow[t]{2}{*}{ Bone } & \multicolumn{3}{|c|}{ Position (m) } \\
\hline & & & & $\mathrm{x}$ & $\mathrm{y}$ & $\mathrm{z}$ \\
\hline Longissimus thoracis & 1 & 4 & $\mathrm{~T} 7$ & -0.0335 & -0.0462 & 0.9620 \\
\hline Longissimus thoracis & 1 & 5 & $\mathrm{~T} 7$ & -0.0310 & -0.0410 & 0.9396 \\
\hline Longissimus thoracis & 1 & 6 & $\mathrm{~T} 8$ & -0.0284 & -0.0359 & 0.9172 \\
\hline Longissimus thoracis & 1 & 7 & T9 & -0.0260 & -0.0365 & 0.8971 \\
\hline Longissimus thoracis & 1 & 8 & $\mathrm{~T} 10$ & -0.0238 & -0.0365 & 0.8773 \\
\hline Longissimus thoracis & 1 & 9 & $\mathrm{~T} 11$ & -0.0210 & -0.0395 & 0.8538 \\
\hline Longissimus thoracis & 1 & 10 & $\mathrm{~T} 12$ & -0.0181 & -0.0425 & 0.8282 \\
\hline Longissimus thoracis & 1 & 11 & $\mathrm{~L} 1$ & -0.0151 & -0.0463 & 0.8014 \\
\hline Longissimus thoracis & 1 & 12 & L2 & -0.0122 & -0.0481 & 0.7772 \\
\hline Longissimus thoracis & 1 & 13 & L3 & -0.0100 & -0.0491 & 0.7572 \\
\hline Longissimus thoracis & 2 & 1 & $\mathrm{~T} 7$ & -0.0386 & -0.0450 & 0.9590 \\
\hline Longissimus thoracis & 2 & 2 & $\mathrm{~T} 8$ & -0.0353 & -0.0450 & 0.9300 \\
\hline Longissimus thoracis & 2 & 3 & T9 & -0.0315 & -0.0365 & 0.8971 \\
\hline Longissimus thoracis & 2 & 4 & $\mathrm{~T} 10$ & -0.0292 & -0.0365 & 0.8773 \\
\hline Longissimus thoracis & 2 & 5 & $\mathrm{~T} 11$ & -0.0266 & -0.0395 & 0.8538 \\
\hline Longissimus thoracis & 2 & 6 & $\mathrm{~T} 12$ & -0.0236 & -0.0425 & 0.8282 \\
\hline Longissimus thoracis & 2 & 7 & $\mathrm{~L} 1$ & -0.0205 & -0.0463 & 0.8014 \\
\hline Longissimus thoracis & 2 & 8 & L2 & -0.0178 & -0.0481 & 0.7772 \\
\hline Longissimus thoracis & 2 & 9 & L3 & -0.0155 & -0.0491 & 0.7572 \\
\hline Longissimus thoracis & 2 & 10 & L4 & -0.0127 & -0.0472 & 0.7329 \\
\hline Longissimus thoracis & 2 & 11 & L5 & -0.0105 & -0.0445 & 0.7140 \\
\hline Longissimus thoracis & 3 & 1 & $\mathrm{~T} 7$ & -0.0438 & -0.0439 & 0.9532 \\
\hline Longissimus thoracis & 3 & 2 & $\mathrm{~T} 8$ & -0.0402 & -0.0445 & 0.9262 \\
\hline Longissimus thoracis & 3 & 3 & T9 & -0.0362 & -0.0490 & 0.8980 \\
\hline Longissimus thoracis & 3 & 4 & $\mathrm{~T} 10$ & -0.0335 & -0.0365 & 0.8773 \\
\hline Longissimus thoracis & 3 & 5 & $\mathrm{~T} 11$ & -0.0303 & -0.0395 & 0.8538 \\
\hline Longissimus thoracis & 3 & 6 & $\mathrm{~T} 12$ & -0.0267 & -0.0425 & 0.8282 \\
\hline Longissimus thoracis & 3 & 7 & L1 & -0.0230 & -0.0463 & 0.8014 \\
\hline Longissimus thoracis & 3 & 8 & L2 & -0.0198 & -0.0481 & 0.7772 \\
\hline Longissimus thoracis & 3 & 9 & L3 & -0.0170 & -0.0491 & 0.7572 \\
\hline Longissimus thoracis & 3 & 10 & L4 & -0.0137 & -0.0472 & 0.7329 \\
\hline Longissimus thoracis & 3 & 11 & L5 & -0.0111 & -0.0445 & 0.7140 \\
\hline Longissimus thoracis & 4 & 1 & T5 & -0.0467 & -0.0545 & 1.0047 \\
\hline Longissimus thoracis & 4 & 2 & T6 & -0.0433 & -0.0485 & 0.9790 \\
\hline Longissimus thoracis & 4 & 3 & $\mathrm{~T} 7$ & -0.0405 & -0.0455 & 0.9580 \\
\hline Longissimus thoracis & 4 & 4 & $\mathrm{~T} 8$ & -0.0365 & -0.0442 & 0.9280 \\
\hline Longissimus thoracis & 4 & 5 & T9 & -0.0319 & -0.0493 & 0.8930 \\
\hline Longissimus thoracis & 4 & 6 & $\mathrm{~T} 10$ & -0.0298 & -0.0365 & 0.8773 \\
\hline Longissimus thoracis & 4 & 7 & $\mathrm{~T} 11$ & -0.0267 & -0.0395 & 0.8538 \\
\hline Longissimus thoracis & 4 & 8 & $\mathrm{~T} 12$ & -0.0234 & -0.0425 & 0.8282 \\
\hline Longissimus thoracis & 4 & 9 & $\mathrm{~L} 1$ & -0.0198 & -0.0463 & 0.8014 \\
\hline Longissimus thoracis & 4 & 10 & $\mathrm{~L} 2$ & -0.0165 & -0.0481 & 0.7772 \\
\hline Longissimus thoracis & 4 & 11 & L3 & -0.0140 & -0.0491 & 0.7572 \\
\hline Longissimus thoracis & 4 & 12 & L4 & -0.0108 & -0.0472 & 0.7329 \\
\hline Longissimus thoracis & 5 & 1 & T9 & -0.0439 & -0.0438 & 0.9255 \\
\hline Longissimus thoracis & 5 & 2 & $\mathrm{~T} 10$ & -0.0389 & -0.0495 & 0.8920 \\
\hline Longissimus thoracis & 5 & 3 & $\mathrm{~T} 11$ & -0.0333 & -0.0395 & 0.8538 \\
\hline Longissimus thoracis & 5 & 4 & $\mathrm{~T} 12$ & -0.0295 & -0.0425 & 0.8282 \\
\hline Longissimus thoracis & 5 & 5 & $\mathrm{~L} 1$ & -0.0255 & -0.0463 & 0.8014 \\
\hline Longissimus thoracis & 5 & 6 & L2 & -0.0220 & -0.0481 & 0.7772 \\
\hline Longissimus thoracis & 5 & 7 & L3 & -0.0190 & -0.0491 & 0.7572 \\
\hline Longissimus thoracis & 5 & 8 & L4 & -0.0155 & -0.0472 & 0.7329 \\
\hline Longissimus thoracis & 5 & 9 & L5 & -0.0127 & -0.0445 & 0.7140 \\
\hline Longissimus thoracis & 5 & 10 & Sacrum & -0.0087 & -0.0370 & 0.6866 \\
\hline Longissimus thoracis & 6 & 1 & $\mathrm{~T} 6$ & -0.0482 & -0.0470 & 0.9762 \\
\hline Longissimus thoracis & 6 & 2 & $\mathrm{~T} 7$ & -0.0440 & -0.0430 & 0.9475 \\
\hline Longissimus thoracis & 6 & 3 & $\mathrm{~T} 8$ & -0.0402 & -0.0450 & 0.9210 \\
\hline Longissimus thoracis & 6 & 4 & T9 & -0.0367 & -0.0490 & 0.8971 \\
\hline Longissimus thoracis & 6 & 5 & $\mathrm{~T} 10$ & -0.0340 & -0.0365 & 0.8773 \\
\hline Longissimus thoracis & 6 & 6 & $\mathrm{~T} 11$ & -0.0305 & -0.0395 & 0.8538 \\
\hline Longissimus thoracis & 6 & 7 & $\mathrm{~T} 12$ & -0.0270 & -0.0425 & 0.8282 \\
\hline
\end{tabular}

Continued on next page 


\begin{tabular}{|c|c|c|c|c|c|c|}
\hline \multirow[t]{2}{*}{ Muscle } & \multirow[t]{2}{*}{ \# } & \multirow[t]{2}{*}{ Via } & \multirow[t]{2}{*}{ Bone } & \multicolumn{3}{|c|}{ Position (m) } \\
\hline & & & & $\mathrm{x}$ & $\mathrm{y}$ & $\mathrm{z}$ \\
\hline Longissimus thoracis & 6 & 8 & $\mathrm{~L} 1$ & -0.0230 & -0.0463 & 0.8014 \\
\hline Longissimus thoracis & 6 & 9 & $\mathrm{~L} 2$ & -0.0195 & -0.0481 & 0.7772 \\
\hline Longissimus thoracis & 6 & 10 & L3 & -0.0167 & -0.0491 & 0.7572 \\
\hline Longissimus thoracis & 6 & 11 & L4 & -0.0132 & -0.0472 & 0.7329 \\
\hline Longissimus thoracis & 6 & 12 & L5 & -0.0105 & -0.0445 & 0.7140 \\
\hline Longissimus thoracis & 12 & 1 & $\mathrm{~T} 12$ & -0.0240 & -0.0425 & 0.8282 \\
\hline Longissimus thoracis & 12 & 2 & $\mathrm{~L} 1$ & -0.0270 & -0.0463 & 0.8014 \\
\hline Longissimus thoracis & 12 & 3 & $\mathrm{~L} 2$ & -0.0297 & -0.0481 & 0.7772 \\
\hline Longissimus thoracis & 12 & 4 & L3 & -0.0320 & -0.0491 & 0.7572 \\
\hline Longissimus thoracis & 12 & 5 & L4 & -0.0347 & -0.0472 & 0.7329 \\
\hline Longissimus thoracis & 12 & 6 & L5 & -0.0369 & -0.0445 & 0.7140 \\
\hline Longissimus thoracis & 12 & 7 & Sacrum & -0.0400 & -0.0370 & 0.6866 \\
\hline Longissimus thoracis & 13 & 1 & $\mathrm{~T} 11$ & -0.0280 & -0.0395 & 0.8538 \\
\hline Longissimus thoracis & 13 & 2 & $\mathrm{~T} 12$ & -0.0296 & -0.0425 & 0.8282 \\
\hline Longissimus thoracis & 13 & 3 & $\mathrm{~L} 1$ & -0.0310 & -0.0463 & 0.8014 \\
\hline Longissimus thoracis & 13 & 4 & L2 & -0.0323 & -0.0481 & 0.7772 \\
\hline Longissimus thoracis & 13 & 5 & L3 & -0.0334 & -0.0491 & 0.7572 \\
\hline Longissimus thoracis & 13 & 6 & L4 & -0.0348 & -0.0472 & 0.7329 \\
\hline Longissimus thoracis & 13 & 7 & L5 & -0.0358 & -0.0445 & 0.7140 \\
\hline Longissimus thoracis & 13 & 8 & Sacrum & -0.0373 & -0.0370 & 0.6866 \\
\hline Longissimus thoracis & 14 & 1 & $\mathrm{~T} 10$ & -0.0292 & -0.0365 & 0.8773 \\
\hline Longissimus thoracis & 14 & 2 & $\mathrm{~T} 11$ & -0.0288 & -0.0395 & 0.8538 \\
\hline Longissimus thoracis & 14 & 3 & $\mathrm{~T} 12$ & -0.0284 & -0.0425 & 0.8282 \\
\hline Longissimus thoracis & 14 & 4 & $\mathrm{~L} 1$ & -0.0278 & -0.0463 & 0.8014 \\
\hline Longissimus thoracis & 14 & 5 & L2 & -0.0276 & -0.0481 & 0.7772 \\
\hline Longissimus thoracis & 14 & 6 & L3 & -0.0272 & -0.0491 & 0.7572 \\
\hline Longissimus thoracis & 14 & 7 & L4 & -0.0268 & -0.0472 & 0.7329 \\
\hline Longissimus thoracis & 14 & 8 & L5 & -0.0265 & -0.0445 & 0.7140 \\
\hline Longissimus thoracis & 14 & 9 & Sacrum & -0.0261 & -0.0370 & 0.6866 \\
\hline Longissimus thoracis & 15 & 1 & $\mathrm{~T} 9$ & -0.0270 & -0.0365 & 0.8971 \\
\hline Longissimus thoracis & 15 & 2 & $\mathrm{~T} 10$ & -0.0252 & -0.0365 & 0.8773 \\
\hline Longissimus thoracis & 15 & 3 & $\mathrm{~T} 11$ & -0.0229 & -0.0395 & 0.8538 \\
\hline Longissimus thoracis & 15 & 4 & $\mathrm{~T} 12$ & -0.0204 & -0.0425 & 0.8282 \\
\hline Longissimus thoracis & 15 & 5 & $\mathrm{~L} 1$ & -0.0179 & -0.0463 & 0.8014 \\
\hline Longissimus thoracis & 15 & 6 & L2 & -0.0155 & -0.0481 & 0.7772 \\
\hline Longissimus thoracis & 15 & 7 & L3 & -0.0137 & -0.0491 & 0.7572 \\
\hline Longissimus thoracis & 15 & 8 & L4 & -0.0113 & -0.0472 & 0.7329 \\
\hline Longissimus thoracis & 15 & 9 & L5 & -0.0096 & -0.0445 & 0.7140 \\
\hline Longissimus thoracis & 15 & 10 & Sacrum & -0.0070 & -0.0370 & 0.6866 \\
\hline Longissimus thoracis & 16 & 1 & $\mathrm{~T} 8$ & -0.0278 & -0.0359 & 0.9172 \\
\hline Longissimus thoracis & 16 & 2 & T9 & -0.0260 & -0.0365 & 0.8971 \\
\hline Longissimus thoracis & 16 & 3 & $\mathrm{~T} 10$ & -0.0242 & -0.0365 & 0.8773 \\
\hline Longissimus thoracis & 16 & 4 & $\mathrm{~T} 11$ & -0.0222 & -0.0395 & 0.8538 \\
\hline Longissimus thoracis & 16 & 5 & $\mathrm{~T} 12$ & -0.0198 & -0.0425 & 0.8282 \\
\hline Longissimus thoracis & 16 & 6 & $\mathrm{~L} 1$ & -0.0175 & -0.0463 & 0.8014 \\
\hline Longissimus thoracis & 16 & 7 & $\mathrm{~L} 2$ & -0.0153 & -0.0481 & 0.7772 \\
\hline Longissimus thoracis & 16 & 8 & L3 & -0.0136 & -0.0491 & 0.7572 \\
\hline Longissimus thoracis & 16 & 9 & $\mathrm{~L} 4$ & -0.0115 & -0.0472 & 0.7329 \\
\hline Longissimus thoracis & 16 & 10 & L5 & -0.0098 & -0.0445 & 0.7140 \\
\hline Longissimus thoracis & 17 & 1 & $\mathrm{~T} 7$ & -0.0280 & -0.0365 & 0.9412 \\
\hline Longissimus thoracis & 17 & 2 & $\mathrm{~T} 8$ & -0.0261 & -0.0359 & 0.9172 \\
\hline Longissimus thoracis & 17 & 3 & T9 & -0.0244 & -0.0365 & 0.8971 \\
\hline Longissimus thoracis & 17 & 4 & $\mathrm{~T} 10$ & -0.0227 & -0.0365 & 0.8773 \\
\hline Longissimus thoracis & 17 & 5 & $\mathrm{~T} 11$ & -0.0208 & -0.0395 & 0.8538 \\
\hline Longissimus thoracis & 17 & 6 & $\mathrm{~T} 12$ & -0.0187 & -0.0425 & 0.8282 \\
\hline Longissimus thoracis & 17 & 7 & $\mathrm{~L} 1$ & -0.0165 & -0.0463 & 0.8014 \\
\hline Longissimus thoracis & 17 & 8 & L2 & -0.0145 & -0.0481 & 0.7772 \\
\hline Longissimus thoracis & 17 & 9 & L3 & -0.0128 & -0.0491 & 0.7572 \\
\hline Longissimus thoracis & 17 & 10 & $\mathrm{~L} 4$ & -0.0108 & -0.0472 & 0.7329 \\
\hline Longissimus thoracis & 17 & 11 & L5 & -0.0092 & -0.0445 & 0.7140 \\
\hline Longissimus thoracis & 18 & 1 & $\mathrm{~T} 6$ & -0.0290 & -0.0405 & 0.9749 \\
\hline
\end{tabular}

Continued on next page 


\begin{tabular}{|c|c|c|c|c|c|c|}
\hline \multirow[t]{2}{*}{ Muscle } & \multirow[t]{2}{*}{ \# } & \multirow[t]{2}{*}{ Via } & \multirow[t]{2}{*}{ Bone } & \multicolumn{3}{|c|}{ Position (m) } \\
\hline & & & & $\mathrm{x}$ & $\mathrm{y}$ & $\mathrm{z}$ \\
\hline Longissimus thoracis & 18 & 2 & $\mathrm{~T} 7$ & -0.0263 & -0.0365 & 0.9412 \\
\hline Longissimus thoracis & 18 & 3 & $\mathrm{~T} 8$ & -0.0244 & -0.0359 & 0.9172 \\
\hline Longissimus thoracis & 18 & 4 & T9 & -0.0228 & -0.0365 & 0.8971 \\
\hline Longissimus thoracis & 18 & 5 & $\mathrm{~T} 10$ & -0.0212 & -0.0365 & 0.8773 \\
\hline Longissimus thoracis & 18 & 6 & $\mathrm{~T} 11$ & -0.0193 & -0.0395 & 0.8538 \\
\hline Longissimus thoracis & 18 & 7 & $\mathrm{~T} 12$ & -0.0174 & -0.0425 & 0.8282 \\
\hline Longissimus thoracis & 18 & 8 & L1 & -0.0152 & -0.0463 & 0.8014 \\
\hline Longissimus thoracis & 18 & 9 & $\mathrm{~L} 2$ & -0.0132 & -0.0481 & 0.7772 \\
\hline Longissimus thoracis & 18 & 10 & L3 & -0.0115 & -0.0491 & 0.7572 \\
\hline Longissimus thoracis & 18 & 11 & $\mathrm{~L} 4$ & -0.0097 & -0.0472 & 0.7329 \\
\hline Longissimus thoracis & 19 & 1 & $\mathrm{~T} 6$ & -0.0323 & -0.0630 & 1.0200 \\
\hline Longissimus thoracis & 19 & 2 & $\mathrm{~T} 7$ & -0.0285 & -0.0405 & 0.9749 \\
\hline Longissimus thoracis & 19 & 3 & $\mathrm{~T} 7$ & -0.0254 & -0.0365 & 0.9412 \\
\hline Longissimus thoracis & 19 & 4 & $\mathrm{~T} 8$ & -0.0233 & -0.0359 & 0.9172 \\
\hline Longissimus thoracis & 19 & 5 & T9 & -0.0215 & -0.0365 & 0.8971 \\
\hline Longissimus thoracis & 19 & 6 & $\mathrm{~T} 10$ & -0.0198 & -0.0365 & 0.8773 \\
\hline Longissimus thoracis & 19 & 7 & $\mathrm{~T} 11$ & -0.0177 & -0.0395 & 0.8538 \\
\hline Longissimus thoracis & 19 & 8 & $\mathrm{~T} 12$ & -0.0154 & -0.0425 & 0.8282 \\
\hline Longissimus thoracis & 19 & 9 & L1 & -0.0132 & -0.0463 & 0.8014 \\
\hline Longissimus thoracis & 19 & 10 & L2 & -0.0110 & -0.0481 & 0.7772 \\
\hline Longissimus thoracis & 19 & 11 & L3 & -0.0092 & -0.0491 & 0.7572 \\
\hline Longissimus thoracis & 20 & 1 & $\mathrm{~T} 4$ & -0.0267 & -0.0657 & 1.0357 \\
\hline Longissimus thoracis & 20 & 2 & T5 & -0.0248 & -0.0494 & 1.0057 \\
\hline Longissimus thoracis & 20 & 3 & $\mathrm{~T} 6$ & -0.0228 & -0.0405 & 0.9749 \\
\hline Longissimus thoracis & 20 & 4 & $\mathrm{~T} 7$ & -0.0205 & -0.0365 & 0.9412 \\
\hline Longissimus thoracis & 20 & 5 & $\mathrm{~T} 8$ & -0.0190 & -0.0359 & 0.9172 \\
\hline Longissimus thoracis & 20 & 6 & T9 & -0.0177 & -0.0365 & 0.8971 \\
\hline Longissimus thoracis & 20 & 7 & $\mathrm{~T} 10$ & -0.0164 & -0.0365 & 0.8773 \\
\hline Longissimus thoracis & 20 & 8 & $\mathrm{~T} 11$ & -0.0150 & -0.0395 & 0.8538 \\
\hline Longissimus thoracis & 20 & 9 & $\mathrm{~T} 12$ & -0.0134 & -0.0425 & 0.8282 \\
\hline Longissimus thoracis & 20 & 10 & $\mathrm{~L} 1$ & -0.0115 & -0.0463 & 0.8014 \\
\hline Longissimus thoracis & 20 & 11 & $\mathrm{~L} 2$ & -0.0100 & -0.0481 & 0.7772 \\
\hline Longissimus thoracis & 20 & 12 & L3 & -0.0086 & -0.0491 & 0.7572 \\
\hline Longissimus thoracis & 21 & 1 & $\mathrm{~T} 4$ & -0.0232 & -0.0787 & 1.0599 \\
\hline Longissimus thoracis & 21 & 2 & T5 & -0.0206 & -0.0494 & 1.0057 \\
\hline Longissimus thoracis & 21 & 3 & $\mathrm{~T} 6$ & -0.0190 & -0.0405 & 0.9749 \\
\hline Longissimus thoracis & 21 & 4 & $\mathrm{~T} 7$ & -0.0175 & -0.0365 & 0.9412 \\
\hline Longissimus thoracis & 21 & 5 & $\mathrm{~T} 8$ & -0.0164 & -0.0359 & 0.9172 \\
\hline Longissimus thoracis & 21 & 6 & T9 & -0.0154 & -0.0365 & 0.8971 \\
\hline Longissimus thoracis & 21 & 7 & $\mathrm{~T} 10$ & -0.0144 & -0.0365 & 0.8773 \\
\hline Longissimus thoracis & 21 & 8 & $\mathrm{~T} 11$ & -0.0134 & -0.0395 & 0.8538 \\
\hline Longissimus thoracis & 21 & 9 & $\mathrm{~T} 12$ & -0.0122 & -0.0425 & 0.8282 \\
\hline Longissimus thoracis & 21 & 10 & L1 & -0.0108 & -0.0463 & 0.8014 \\
\hline Longissimus thoracis & 21 & 11 & L2 & -0.0096 & -0.0481 & 0.7772 \\
\hline Longissimus thoracis & 22 & 1 & $\mathrm{~T} 3$ & -0.0204 & -0.1071 & 1.0832 \\
\hline Longissimus thoracis & 22 & 2 & $\mathrm{~T} 4$ & -0.0193 & -0.0787 & 1.0599 \\
\hline Longissimus thoracis & 22 & 3 & T5 & -0.0170 & -0.0494 & 1.0057 \\
\hline Longissimus thoracis & 22 & 4 & $\mathrm{~T} 6$ & -0.0158 & -0.0405 & 0.9749 \\
\hline Longissimus thoracis & 22 & 5 & $\mathrm{~T} 7$ & -0.0143 & -0.0365 & 0.9412 \\
\hline Longissimus thoracis & 22 & 6 & $\mathrm{~T} 8$ & -0.0132 & -0.0359 & 0.9172 \\
\hline Longissimus thoracis & 22 & 7 & T9 & -0.0124 & -0.0365 & 0.8971 \\
\hline Longissimus thoracis & 22 & 8 & $\mathrm{~T} 10$ & -0.0117 & -0.0365 & 0.8773 \\
\hline Longissimus thoracis & 22 & 9 & $\mathrm{~T} 11$ & -0.0106 & -0.0395 & 0.8538 \\
\hline Longissimus thoracis & 22 & 10 & $\mathrm{~T} 12$ & -0.0095 & -0.0425 & 0.8282 \\
\hline Longissimus thoracis & 22 & 11 & L1 & -0.0083 & -0.0463 & 0.8014 \\
\hline Longissimus thoracis & 23 & 1 & $\mathrm{~T} 2$ & -0.0216 & -0.1209 & 1.0976 \\
\hline Longissimus thoracis & 23 & 2 & T3 & -0.0208 & -0.1071 & 1.0832 \\
\hline Longissimus thoracis & 23 & 3 & $\mathrm{~T} 4$ & -0.0197 & -0.0787 & 1.0599 \\
\hline Longissimus thoracis & 23 & 4 & T5 & -0.0169 & -0.0494 & 1.0057 \\
\hline Longissimus thoracis & 23 & 5 & T6 & -0.0153 & -0.0405 & 0.9749 \\
\hline Longissimus thoracis & 23 & 6 & $\mathrm{~T} 7$ & -0.0135 & -0.0365 & 0.9412 \\
\hline
\end{tabular}

Continued on next page 


\begin{tabular}{|c|c|c|c|c|c|c|}
\hline \multirow[t]{2}{*}{ Muscle } & \multirow[t]{2}{*}{ \# } & \multirow[t]{2}{*}{ Via } & \multirow[t]{2}{*}{ Bone } & \multicolumn{3}{|c|}{ Position (m) } \\
\hline & & & & $\mathrm{x}$ & $\mathrm{y}$ & $\mathrm{z}$ \\
\hline Longissimus thoracis & 23 & 7 & $\mathrm{~T} 8$ & -0.0123 & -0.0359 & 0.9172 \\
\hline Longissimus thoracis & 23 & 8 & $\mathrm{~T} 9$ & -0.0113 & -0.0365 & 0.8971 \\
\hline Longissimus thoracis & 23 & 9 & $\mathrm{~T} 10$ & -0.0103 & -0.0365 & 0.8773 \\
\hline Longissimus thoracis & 23 & 10 & $\mathrm{~T} 11$ & -0.0090 & -0.0395 & 0.8538 \\
\hline Longissimus thoracis & 23 & 11 & $\mathrm{~T} 12$ & -0.0078 & -0.0425 & 0.8282 \\
\hline Psoas major & 1 & 1 & Pelvis & -0.0738 & -0.1499 & 0.6250 \\
\hline Psoas major & 2 & 1 & Pelvis & -0.0738 & -0.1499 & 0.6250 \\
\hline Psoas major & 2 & 2 & L5 & -0.0510 & -0.1290 & 0.6800 \\
\hline Psoas major & 2 & 3 & L4 & -0.0366 & -0.1158 & 0.7150 \\
\hline Psoas major & 3 & 1 & Pelvis & -0.0738 & -0.1499 & 0.6250 \\
\hline Psoas major & 3 & 2 & L5 & -0.0573 & -0.1262 & 0.6800 \\
\hline Psoas major & 3 & 3 & L4 & -0.0469 & -0.1113 & 0.7150 \\
\hline Psoas major & 4 & 1 & Pelvis & -0.0738 & -0.1499 & 0.6250 \\
\hline Psoas major & 4 & 2 & L5 & -0.0523 & -0.1215 & 0.6800 \\
\hline Psoas major & 5 & 1 & Pelvis & -0.0738 & -0.1499 & 0.6250 \\
\hline Psoas major & 5 & 2 & L5 & -0.0577 & -0.1300 & 0.6800 \\
\hline Psoas major & 5 & 3 & L4 & -0.0475 & -0.1174 & 0.7150 \\
\hline Psoas major & 5 & 4 & L3 & -0.0359 & -0.1030 & 0.7550 \\
\hline Psoas major & 6 & 1 & Pelvis & -0.0738 & -0.1499 & 0.6250 \\
\hline Psoas major & 6 & 2 & L5 & -0.0585 & -0.1328 & 0.6800 \\
\hline Psoas major & 6 & 3 & L4 & -0.0485 & -0.1220 & 0.7150 \\
\hline Psoas major & 6 & 4 & L3 & -0.0372 & -0.1097 & 0.7550 \\
\hline Psoas major & 6 & 5 & L2 & -0.0283 & -0.0998 & 0.7870 \\
\hline Psoas major & 7 & 1 & Pelvis & -0.0738 & -0.1499 & 0.6250 \\
\hline Psoas major & 7 & 2 & L5 & -0.0600 & -0.1360 & 0.6800 \\
\hline Psoas major & 7 & 3 & L4 & -0.0513 & -0.1272 & 0.7150 \\
\hline Psoas major & 7 & 4 & L3 & -0.0414 & -0.1170 & 0.7550 \\
\hline Psoas major & 7 & 5 & L2 & -0.0334 & -0.1088 & 0.7870 \\
\hline Psoas major & 8 & 1 & Pelvis & -0.0738 & -0.1499 & 0.6250 \\
\hline Psoas major & 8 & 2 & L5 & -0.0513 & -0.1310 & 0.6800 \\
\hline Psoas major & 8 & 3 & L4 & -0.0374 & -0.1190 & 0.7150 \\
\hline Psoas major & 9 & 1 & Pelvis & -0.0738 & -0.1499 & 0.6250 \\
\hline Psoas major & 9 & 2 & L5 & -0.0560 & -0.1365 & 0.6800 \\
\hline Psoas major & 9 & 3 & L4 & -0.0445 & -0.1280 & 0.7150 \\
\hline Psoas major & 9 & 4 & L3 & -0.0318 & -0.1185 & 0.7550 \\
\hline Psoas major & 10 & 1 & Pelvis & -0.0738 & -0.1499 & 0.6250 \\
\hline Psoas major & 10 & 2 & L5 & -0.0565 & -0.1370 & 0.6800 \\
\hline Psoas major & 10 & 3 & $\mathrm{~L} 4$ & -0.0455 & -0.1287 & 0.7150 \\
\hline Psoas major & 10 & 4 & L3 & -0.0330 & -0.1195 & 0.7550 \\
\hline Transversus abdominis & 2 & 1 & $\mathrm{~L} 4$ & -0.1156 & -0.1596 & 0.7063 \\
\hline Transversus abdominis & 3 & 1 & L4 & -0.0780 & -0.0670 & 0.7450 \\
\hline Transversus abdominis & 3 & 2 & L4 & -0.1052 & -0.0925 & 0.7447 \\
\hline Transversus abdominis & 3 & 3 & L3 & -0.1157 & -0.1100 & 0.7430 \\
\hline Transversus abdominis & 3 & 4 & $\mathrm{~L} 4$ & -0.1166 & -0.1604 & 0.7175 \\
\hline Transversus abdominis & 4 & 1 & L3 & -0.0780 & -0.0660 & 0.7620 \\
\hline Transversus abdominis & 4 & 2 & L3 & -0.1052 & -0.0925 & 0.7560 \\
\hline Transversus abdominis & 4 & 3 & L3 & -0.1157 & -0.1100 & 0.7524 \\
\hline Transversus abdominis & 4 & 4 & $\mathrm{~L} 4$ & -0.1176 & -0.1612 & 0.7288 \\
\hline Transversus abdominis & 5 & 1 & $\mathrm{~L} 2$ & -0.0780 & -0.0630 & 0.7880 \\
\hline Transversus abdominis & 5 & 2 & L3 & -0.1052 & -0.0925 & 0.7700 \\
\hline Transversus abdominis & 5 & 3 & L3 & -0.1167 & -0.1089 & 0.7602 \\
\hline Transversus abdominis & 5 & 4 & L3 & -0.1186 & -0.1619 & 0.7400 \\
\hline Transversus abdominis & 6 & 1 & L1 & -0.0780 & -0.0610 & 0.8160 \\
\hline Transversus abdominis & 6 & 2 & L2 & -0.1052 & -0.0925 & 0.7850 \\
\hline Transversus abdominis & 6 & 3 & L3 & -0.1176 & -0.1077 & 0.7679 \\
\hline Transversus abdominis & 6 & 4 & L3 & -0.1196 & -0.1627 & 0.7513 \\
\hline Transversus abdominis & 7 & 1 & L3 & -0.1206 & -0.1635 & 0.7625 \\
\hline
\end{tabular}




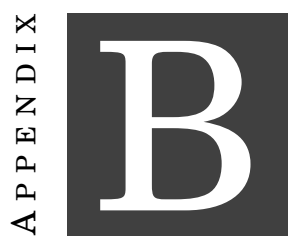

APPENDIX B

\section{B.1 Coordinates of the via attachments}

Table B.1: Coordinates of the via attachments for the muscle elements with a curved lines-of-action. The coordinates are given with respect to the global reference frame.

\begin{tabular}{|c|c|c|c|c|c|c|}
\hline \multirow[t]{2}{*}{ Muscle } & \multirow[t]{2}{*}{ \# } & \multirow[t]{2}{*}{ Via } & \multirow[t]{2}{*}{ Bone } & \multicolumn{3}{|c|}{ Position (m) } \\
\hline & & & & $\mathrm{x}$ & $\mathrm{y}$ & $\mathrm{z}$ \\
\hline Iliocostalis cervicis & 1 & 1 & $\mathrm{R} 1$ & -0.0316 & -0.1504 & 1.1040 \\
\hline Iliocostalis cervicis & 1 & 2 & $\mathrm{R} 2$ & -0.0405 & -0.1248 & 1.0969 \\
\hline Iliocostalis cervicis & 1 & 3 & $\mathrm{R} 3$ & -0.0505 & -0.1008 & 1.0770 \\
\hline Iliocostalis cervicis & 1 & 4 & $\mathrm{R} 4$ & -0.0573 & -0.0811 & 1.0560 \\
\hline Iliocostalis cervicis & 2 & 1 & $\mathrm{R} 1$ & -0.0322 & -0.1520 & 1.1039 \\
\hline Iliocostalis cervicis & 2 & 2 & $\mathrm{R} 2$ & -0.0413 & -0.1222 & 1.0941 \\
\hline Iliocostalis cervicis & 2 & 3 & $\mathrm{R} 3$ & -0.0501 & -0.1015 & 1.0779 \\
\hline Iliocostalis cervicis & 3 & 1 & $\mathrm{R} 1$ & -0.0327 & -0.1511 & 1.1033 \\
\hline Iliocostalis cervicis & 3 & 2 & $\mathrm{R} 2$ & -0.0383 & -0.1235 & 1.0951 \\
\hline Iliocostalis thoracis & 1 & 1 & $\mathrm{R} 6$ & -0.0670 & -0.0595 & 1.0148 \\
\hline Iliocostalis thoracis & 1 & 2 & $\mathrm{R} 7$ & -0.0679 & -0.0535 & 0.9909 \\
\hline Iliocostalist thoracis & 1 & 3 & $\mathrm{R} 8$ & -0.0698 & -0.0477 & 0.9628 \\
\hline Iliocostalis thoracis & 1 & 4 & $\mathrm{R} 9$ & -0.0690 & -0.0452 & 0.9308 \\
\hline Iliocostalis thoracis & 1 & 5 & R10 & -0.0677 & -0.0475 & 0.8994 \\
\hline Iliocostalis thoracis & 2 & 1 & $\mathrm{R} 5$ & -0.0612 & -0.0684 & 1.0378 \\
\hline Iliocostalis thoracis & 2 & 2 & $\mathrm{R} 6$ & -0.0632 & -0.0599 & 1.0160 \\
\hline Iliocostalis thoracis & 2 & 3 & $\mathrm{R} 7$ & -0.0649 & -0.0528 & 0.9899 \\
\hline Iliocostalis thoracis & 2 & 4 & $\mathrm{R} 8$ & -0.0677 & -0.0474 & 0.9642 \\
\hline Iliocostalis thoracis & 2 & 5 & $\mathrm{R} 9$ & -0.0668 & -0.0445 & 0.9330 \\
\hline Iliocostalis thoracis & 3 & 1 & $\mathrm{R} 4$ & -0.0579 & -0.0818 & 1.0569 \\
\hline Iliocostalis thoracis & 3 & 2 & $\mathrm{R} 5$ & -0.0611 & -0.0678 & 1.0356 \\
\hline Iliocostalis thoracis & 3 & 3 & $\mathrm{R} 6$ & -0.0644 & -0.0596 & 1.0150 \\
\hline Iliocostalis thoracis & 3 & 4 & $\mathrm{R} 7$ & -0.0661 & -0.0529 & 0.9898 \\
\hline Iliocostalis thoracis & 3 & 5 & $\mathrm{R} 8$ & -0.0698 & -0.0477 & 0.9628 \\
\hline Omohyoid & 1 & 1 & $\mathrm{~T} 1$ & -0.0411 & -0.1799 & 1.1004 \\
\hline Semispinalis thoracis & 1 & 1 & $\mathrm{~T} 3$ & -0.0084 & -0.0859 & 1.0671 \\
\hline Semispinalis thoracis & 1 & 2 & $\mathrm{~T} 4$ & -0.0141 & -0.0784 & 1.0503 \\
\hline Semispinalis thoracis & 1 & 3 & T5 & -0.0185 & -0.0673 & 1.0269 \\
\hline Semispinalis thoracis & 1 & 4 & $\mathrm{~T} 6$ & -0.0191 & -0.0640 & 1.0103 \\
\hline Semispinalis thoracis & 1 & 5 & $\mathrm{~T} 7$ & -0.0199 & -0.0578 & 0.9851 \\
\hline Semispinalis thoracis & 1 & 6 & $\mathrm{~T} 8$ & -0.0275 & -0.0487 & 0.9556 \\
\hline Semispinalis thoracis & 2 & 1 & $\mathrm{~T} 2$ & -0.0045 & -0.1044 & 1.0888 \\
\hline Semispinalis thoracis & 2 & 2 & $\mathrm{~T} 3$ & -0.0084 & -0.0998 & 1.0746 \\
\hline
\end{tabular}




\begin{tabular}{|c|c|c|c|c|c|c|}
\hline \multirow[t]{2}{*}{ Muscle } & \multirow[t]{2}{*}{ \# } & \multirow[t]{2}{*}{ Via } & \multirow[t]{2}{*}{ Bone } & \multicolumn{3}{|c|}{ Position (m) } \\
\hline & & & & $\mathrm{x}$ & $\mathrm{y}$ & $\mathrm{z}$ \\
\hline Semispinalis thoracis & 2 & 3 & $\mathrm{~T} 4$ & -0.0184 & -0.0812 & 1.0508 \\
\hline Semispinalis thoracis & 2 & 4 & $\mathrm{~T} 5$ & -0.0200 & -0.0684 & 1.0247 \\
\hline Semispinalis thoracis & 2 & 5 & $\mathrm{~T} 6$ & -0.0209 & -0.0624 & 1.0075 \\
\hline Semispinalis thoracis & 2 & 6 & $\mathrm{~T} 7$ & -0.0280 & -0.0524 & 0.9834 \\
\hline Semispinalis thoracis & 3 & 1 & $\mathrm{~T} 1$ & 0.0027 & -0.1160 & 1.1031 \\
\hline Semispinalis thoracis & 3 & 2 & $\mathrm{~T} 2$ & -0.0052 & -0.1120 & 1.0900 \\
\hline Semispinalis thoracis & 3 & 3 & $\mathrm{~T} 3$ & -0.0113 & -0.0954 & 1.0721 \\
\hline Semispinalis thoracis & 3 & 4 & $\mathrm{~T} 4$ & -0.0186 & -0.0824 & 1.0541 \\
\hline Semispinalis thoracis & 3 & 5 & $\mathrm{~T} 5$ & -0.0217 & -0.0656 & 1.0178 \\
\hline Semispinalis thoracis & 3 & 6 & $\mathrm{~T} 6$ & -0.0238 & -0.0634 & 1.0096 \\
\hline Semispinalis thoracis & 4 & 1 & $\mathrm{C} 7$ & 0.0099 & -0.1345 & 1.1154 \\
\hline Semispinalis thoracis & 4 & 2 & $\mathrm{~T} 1$ & 0.0055 & -0.1306 & 1.1100 \\
\hline Semispinalis thoracis & 4 & 3 & $\mathrm{~T} 2$ & -0.0053 & -0.1125 & 1.0907 \\
\hline Semispinalis thoracis & 4 & 4 & $\mathrm{~T} 3$ & -0.0149 & -0.0974 & 1.0709 \\
\hline Semispinalis thoracis & 4 & 5 & $\mathrm{~T} 4$ & -0.0188 & -0.0850 & 1.0542 \\
\hline Semispinalis thoracis & 4 & 6 & $\mathrm{~T} 5$ & -0.0248 & -0.0745 & 1.0376 \\
\hline Spinalis thoracis & 1 & 1 & $\mathrm{~T} 8$ & -0.0118 & -0.0396 & 0.9503 \\
\hline Spinalis thoracis & 1 & 2 & $\mathrm{~T} 9$ & -0.0134 & -0.0382 & 0.9257 \\
\hline Spinalis thoracis & 1 & 3 & $\mathrm{~T} 10$ & -0.0131 & -0.0389 & 0.8958 \\
\hline Spinalis thoracis & 1 & 4 & $\mathrm{~T} 11$ & -0.0129 & -0.0366 & 0.8794 \\
\hline Spinalis thoracis & 1 & 5 & $\mathrm{~T} 12$ & -0.0109 & -0.0402 & 0.8538 \\
\hline Spinalis thoracis & 2 & 1 & $\mathrm{~T} 7$ & -0.0114 & -0.0452 & 0.9833 \\
\hline Spinalis thoracis & 2 & 2 & $\mathrm{~T} 8$ & -0.0136 & -0.0410 & 0.9610 \\
\hline Spinalis thoracis & 2 & 3 & $\mathrm{~T} 9$ & -0.0126 & -0.0403 & 0.9277 \\
\hline Spinalis thoracis & 2 & 4 & $\mathrm{~T} 10$ & -0.0125 & -0.0393 & 0.9038 \\
\hline Spinalis thoracis & 2 & 5 & $\mathrm{~T} 11$ & -0.0119 & -0.0392 & 0.8766 \\
\hline Spinalis thoracis & 2 & 6 & $\mathrm{~T} 12$ & -0.0104 & -0.0415 & 0.8524 \\
\hline Spinalis thoracis & 3 & 1 & $\mathrm{~T} 5$ & -0.0156 & -0.0592 & 1.0169 \\
\hline Spinalis thoracis & 3 & 2 & $\mathrm{~T} 6$ & -0.0223 & -0.0580 & 1.0022 \\
\hline Spinalis thoracis & 3 & 3 & $\mathrm{~T} 7$ & -0.0203 & -0.0520 & 0.9785 \\
\hline Spinalis thoracis & 3 & 4 & $\mathrm{~T} 8$ & -0.0228 & -0.0440 & 0.9559 \\
\hline Spinalis thoracis & 3 & 5 & T9 & -0.0210 & -0.0450 & 0.9201 \\
\hline Spinalis thoracis & 3 & 6 & $\mathrm{~T} 10$ & -0.0180 & -0.0450 & 0.9058 \\
\hline Spinalis thoracis & 3 & 7 & $\mathrm{~T} 11$ & -0.0175 & -0.0439 & 0.8816 \\
\hline Spinalis thoracis & 3 & 8 & $\mathrm{~T} 12$ & -0.0133 & -0.0444 & 0.8455 \\
\hline Spinalis thoracis & 4 & 1 & $\mathrm{~T} 5$ & -0.0158 & -0.0620 & 1.0227 \\
\hline Spinalis thoracis & 4 & 2 & $\mathrm{~T} 6$ & -0.0223 & -0.0570 & 1.0022 \\
\hline Spinalis thoracis & 4 & 3 & $\mathrm{~T} 7$ & -0.0203 & -0.0510 & 0.9785 \\
\hline Spinalis thoracis & 4 & 4 & $\mathrm{~T} 8$ & -0.0228 & -0.0430 & 0.9559 \\
\hline Spinalis thoracis & 4 & 5 & $\mathrm{~T} 9$ & -0.0210 & -0.0450 & 0.9201 \\
\hline Spinalis thoracis & 4 & 6 & $\mathrm{~T} 10$ & -0.0180 & -0.0463 & 0.9058 \\
\hline Spinalis thoracis & 4 & 7 & $\mathrm{~T} 11$ & -0.0175 & -0.0439 & 0.8816 \\
\hline Spinalis thoracis & 4 & 8 & $\mathrm{~T} 12$ & -0.0133 & -0.0444 & 0.8455 \\
\hline Spinalis thoracis & 5 & 1 & $\mathrm{~T} 4$ & -0.0147 & -0.0620 & 1.0311 \\
\hline Spinalis thoracis & 5 & 2 & $\mathrm{~T} 5$ & -0.0223 & -0.0580 & 1.0022 \\
\hline Spinalis thoracis & 5 & 3 & $\mathrm{~T} 6$ & -0.0203 & -0.0512 & 0.9785 \\
\hline Spinalis thoracis & 5 & 4 & $\mathrm{~T} 7$ & -0.0228 & -0.0441 & 0.9559 \\
\hline Spinalis thoracis & 5 & 5 & $\mathrm{~T} 8$ & -0.0219 & -0.0447 & 0.9380 \\
\hline Spinalis thoracis & 5 & 6 & $\mathrm{~T} 9$ & -0.0210 & -0.0474 & 0.9201 \\
\hline Spinalis thoracis & 5 & 7 & $\mathrm{~T} 10$ & -0.0180 & -0.0453 & 0.9058 \\
\hline Spinalis thoracis & 5 & 8 & $\mathrm{~T} 11$ & -0.0175 & -0.0439 & 0.8816 \\
\hline Spinalis thoracis & 5 & 9 & $\mathrm{~T} 12$ & -0.0133 & -0.0444 & 0.8455 \\
\hline Spinalis thoracis & 6 & 1 & T9 & -0.0102 & -0.0389 & 0.9179 \\
\hline Spinalis thoracis & 6 & 2 & $\mathrm{~T} 10$ & -0.0131 & -0.0389 & 0.8958 \\
\hline Spinalis thoracis & 6 & 3 & $\mathrm{~T} 11$ & -0.0129 & -0.0366 & 0.8794 \\
\hline Spinalis thoracis & 6 & 4 & $\mathrm{~T} 12$ & -0.0109 & -0.0402 & 0.8538 \\
\hline
\end{tabular}

\section{B.2 Mathematical definitions of the wrapping surfaces}




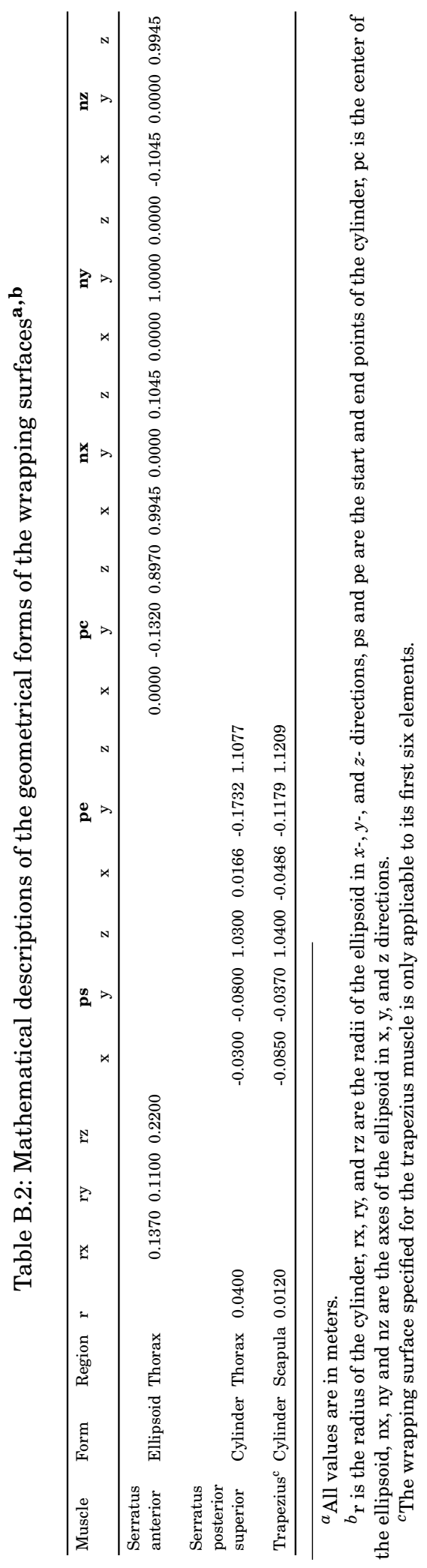




\section{B.3 Architectural tendon parameters}

Table B.3: Architectural tendon parameters for the deeper back muscles ${ }^{\mathrm{a}}$.

\begin{tabular}{|c|c|c|c|c|}
\hline Muscle & $\#$ & $\begin{array}{l}\text { Length } \\
(\mathrm{mm})\end{array}$ & $\begin{array}{l}\text { Mass } \\
(\mathrm{g})\end{array}$ & $\begin{array}{l}\text { Area } \\
\left(\mathrm{cm}^{2}\right)\end{array}$ \\
\hline Levatores costarum & 1 & 28.0 & 0.06 & 0.018 \\
\hline Levatores costarum & 2 & 49.0 & 0.03 & 0.005 \\
\hline Levatores costarum & 3 & 29.0 & 0.06 & 0.017 \\
\hline Levatores costarum & 4 & 29.0 & 0.07 & 0.020 \\
\hline Levatores costarum & 5 & 26.0 & 0.02 & 0.006 \\
\hline Levatores costarum & 6 & 33.0 & 0.05 & 0.013 \\
\hline Levatores costarum & 7 & 39.0 & 0.03 & 0.006 \\
\hline Levatores costarum & 8 & 35.0 & 0.06 & 0.014 \\
\hline Levatores costarum & 9 & 44.0 & 0.05 & 0.009 \\
\hline Levatores costarum & 10 & 59.0 & 0.16 & 0.023 \\
\hline Levatores costarum & 11 & 42.0 & 0.07 & 0.014 \\
\hline Levatores costarum & 12 & 72.0 & 0.08 & 0.009 \\
\hline Levatores costarum & 13 & 40.0 & 0.04 & 0.008 \\
\hline Multifidus & 19 & 95.0 & 0.09 & 0.008 \\
\hline Multifidus & 20 & 113.5 & 0.37 & 0.027 \\
\hline Multifidus & 21 & 139.0 & 0.26 & 0.016 \\
\hline Multifidus & 22 & 41.0 & 0.02 & 0.004 \\
\hline Multifidus & 23 & 53.0 & 0.02 & 0.003 \\
\hline Multifidus & 24 & 102.0 & 0.07 & 0.006 \\
\hline Multifidus & 25 & 79.0 & 0.04 & 0.004 \\
\hline Multifidus & 26 & 47.0 & 0.09 & 0.016 \\
\hline Multifidus & 27 & 57.0 & 0.02 & 0.003 \\
\hline Multifidus & 28 & 84.5 & 0.08 & 0.008 \\
\hline Multifidus & 29 & 39.5 & 0.08 & 0.017 \\
\hline Multifidus & 30 & 61.5 & 0.04 & 0.005 \\
\hline Multifidus & 31 & 35.5 & 0.01 & 0.002 \\
\hline Multifidus & 32 & 47.0 & 0.01 & 0.002 \\
\hline Multifidus & 33 & 66.0 & 0.16 & 0.020 \\
\hline Multifidus & 34 & 65.5 & 0.07 & 0.009 \\
\hline Multifidus & 35 & 93.0 & 0.08 & 0.007 \\
\hline Multifidus & 36 & 70.0 & 0.09 & 0.011 \\
\hline Multifidus & 37 & 37.0 & 0.03 & 0.007 \\
\hline Multifidus & 38 & 44.0 & 0.09 & 0.017 \\
\hline Multifidus & 39 & 44.0 & 0.06 & 0.011 \\
\hline Multifidus & 40 & 105.0 & 0.17 & 0.013 \\
\hline Multifidus & 41 & 67.0 & 0.18 & 0.022 \\
\hline Multifidus & 42 & 44.0 & 0.03 & 0.006 \\
\hline Multifidus & 43 & 85.0 & 0.03 & 0.003 \\
\hline Multifidus & 44 & 100.5 & 0.05 & 0.004 \\
\hline Multifidus & 45 & 48.5 & 0.05 & 0.009 \\
\hline Multifidus & 46 & 76.5 & 0.15 & 0.016 \\
\hline Multifidus & 47 & 79.0 & 0.06 & 0.006 \\
\hline Multifidus & 48 & 101.0 & 0.04 & 0.003 \\
\hline Multifidus & 49 & 76.5 & 0.11 & 0.012 \\
\hline Multifidus & 50 & 49.0 & 0.04 & 0.007 \\
\hline Multifidus & 51 & 45.0 & 0.06 & 0.011 \\
\hline Multifidus & 52 & 61.0 & 0.06 & 0.008 \\
\hline Multifidus & 53 & 37.5 & 0.02 & 0.004 \\
\hline Multifidus & 54 & 66.0 & 0.15 & 0.019 \\
\hline
\end{tabular}

Continued on next page 
Table B.3: Continued from previous page

\begin{tabular}{|c|c|c|c|c|}
\hline Muscle & \# & $\begin{array}{l}\text { Length } \\
(\mathrm{mm})\end{array}$ & $\begin{array}{l}\text { Mass } \\
(\mathrm{g})\end{array}$ & $\begin{array}{l}\text { CSA } \\
\left(\mathrm{cm}^{2}\right)\end{array}$ \\
\hline Multifidus & 55 & 43.0 & 0.01 & 0.002 \\
\hline Multifidus & 56 & 47.0 & 0.02 & 0.004 \\
\hline Multifidus & 57 & 39.5 & 0.04 & 0.008 \\
\hline Multifidus & 58 & 34.0 & 0.21 & 0.051 \\
\hline Multifidus & 59 & 48.0 & 0.04 & 0.007 \\
\hline Multifidus & 60 & 44.5 & 0.21 & 0.039 \\
\hline Multifidus & 61 & 33.0 & 0.13 & 0.033 \\
\hline Multifidus & 62 & 39.0 & 0.1 & 0.021 \\
\hline Multifidus & 63 & 36.5 & 0.06 & 0.014 \\
\hline Multifidus & 64 & 39.0 & 0.01 & 0.002 \\
\hline Rotatores & 1 & 30.0 & 0.02 & 0.006 \\
\hline Rotatores & 2 & 30.0 & 0.02 & 0.006 \\
\hline Rotatores & 3 & 26.0 & 0.09 & 0.029 \\
\hline Rotatores & 4 & 23.0 & 0.03 & 0.011 \\
\hline Rotatores & 5 & 18.0 & 0.01 & 0.005 \\
\hline Rotatores & 6 & 20.0 & 0.01 & 0.004 \\
\hline Rotatores & 7 & 26.0 & 0.01 & 0.003 \\
\hline Rotatores & 8 & 13.0 & 0.02 & 0.013 \\
\hline Rotatores & 9 & 15.0 & 0.01 & 0.006 \\
\hline Rotatores & 10 & 8.0 & 0.03 & 0.031 \\
\hline Rotatores & 11 & 24.0 & 0.03 & 0.010 \\
\hline Rotatores & 12 & 8.0 & 0.02 & 0.021 \\
\hline Rotatores & 13 & 26.5 & 0.02 & 0.006 \\
\hline Rotatores & 14 & 9.0 & 0.03 & 0.028 \\
\hline Rotatores & 15 & 38.0 & 0.03 & 0.007 \\
\hline Rotatores & 16 & 9.0 & 0.02 & 0.018 \\
\hline Rotatores & 17 & 27.5 & 0.02 & 0.006 \\
\hline Rotatores & 18 & 7.0 & 0.01 & 0.012 \\
\hline Rotatores & 19 & 26.5 & 0.01 & 0.003 \\
\hline Rotatores & 20 & 31.5 & 0.02 & 0.005 \\
\hline Rotatores & 21 & 27.0 & 0.02 & 0.006 \\
\hline Rotatores & 22 & 19.0 & 0.01 & 0.004 \\
\hline Rotatores & 23 & 30.5 & 0.08 & 0.022 \\
\hline Rotatores & 24 & 30.5 & 0.08 & 0.022 \\
\hline Rotatores & 25 & 21.0 & 0.04 & 0.016 \\
\hline Rotatores & 26 & 27.5 & 0.03 & 0.009 \\
\hline Spinalis thoracis & 1 & 110.0 & 0.07 & 0.005 \\
\hline Spinalis thoracis & 2 & 140.0 & 0.07 & 0.004 \\
\hline Spinalis thoracis & 3 & 186.0 & 0.15 & 0.007 \\
\hline Spinalis thoracis & 4 & 219.5 & 0.15 & 0.006 \\
\hline Spinalis thoracis & 5 & 224.0 & 0.15 & 0.006 \\
\hline Spinalis thoracis & 6 & 101.5 & 0.07 & 0.006 \\
\hline
\end{tabular}

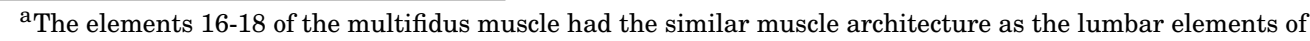
this muscle (tendon-muscle-tendon architecture). Therefore, we did not measure mass and cross-sectional area of their tendons. 


\section{B.4 Abbreviations used for the muscles}

Table B.4: Abbreviations used for the muscles illustrated in Fig. 3.4

\begin{tabular}{|c|c|}
\hline Muscle name & Abbreviation \\
\hline Iliocostalis cervicis & ICE \\
\hline Iliocostalis thoracis & IT \\
\hline Intercostales externi & IE \\
\hline Intercostales interni & II \\
\hline Levator scapulae & LS \\
\hline Levatores costarum & LEC \\
\hline Longissimus capitis & LCA \\
\hline Longisssimus cervicis & LCE \\
\hline Longus capitis & LUCA \\
\hline Multifidus thoracic & MFT \\
\hline Obliquus capitis inferior & OCI \\
\hline Obliquus capitis superior & OCS \\
\hline Omohyoid & $\mathrm{OH}$ \\
\hline Rectus capitis anterior & $\mathrm{RCA}$ \\
\hline Rectus capitis lateralis & RCL \\
\hline Rectus capitis posterior major & RCPMA \\
\hline Rectus capitis posterior minor & RCPMI \\
\hline Rhomboideus (major and minor) & $\mathrm{RM}$ \\
\hline Rotatores & ROT \\
\hline Scalenus anterior & SAN \\
\hline Scalenus medius & SM \\
\hline Scalenus posterior & SP \\
\hline Semispinalis capitis & SSCA \\
\hline Semispinalis cervicis & SSCE \\
\hline Semispinalis thoracis & SST \\
\hline Serratus anterior & SA \\
\hline Serratus posterior superior & SPS \\
\hline Spinalis thoracis & ST \\
\hline Splenius capitis & SUCA \\
\hline Splenius cervicis & SCE \\
\hline Sternocleidomastoid & STCM \\
\hline Sternohyoid & STH \\
\hline Sternothyroid & STT \\
\hline Subclavious & SCL \\
\hline Subcostales & $\mathrm{SCO}$ \\
\hline Thyrohyoid & TH \\
\hline Transversus thoracis & TT \\
\hline Trapezius & $\mathrm{TP}$ \\
\hline Iliocostalis lumborum & IL \\
\hline Latissimus dorsi & $\mathrm{LD}$ \\
\hline Longissimus thoracis & LT \\
\hline Multifidus lumbar & MFL \\
\hline Obliquus externus abdominis & OEA \\
\hline Obliquus internus abdominis & OIA \\
\hline Psoas major & PM \\
\hline Quadratus lumborum & $\mathrm{QL}$ \\
\hline Rectus abdominis & RA \\
\hline Serratus posterior inferior & SPI \\
\hline Transversus abdominis & $\mathrm{TA}$ \\
\hline
\end{tabular}




\section{BIBLIOGRAPHY}

[1] M. Dreischarf, A. Shirazi-Adl, N. Arjmand, A. Rohlmann, and H. Schmidt, "Estimation of loads on human lumbar spine: a review of in vivo and computational model studies," Journal of biomechanics, vol. 49, no. 6, pp. 833-845, 2016.

[2] F. Hug and K. Tucker, "Muscle coordination and the development of musculoskeletal disorders," Exercise and sport sciences reviews, vol. 45, no. 4, pp. 201-208, 2017.

[3] A. Nachemson and G. Elfstrom, "Intravital dynamic pressure measurements in lumbar discs," Scand J Rehabil Med, vol. 2, no. suppl 1, pp. 1-40, 1970.

[4] A. Schultz, G. Andersson, R. Ortengren, K. Haderspeck, and A. Nachemson, "Loads on the lumbar spine. validation of a biomechanical analysis by measurements of intradiscal pressures and myoelectric signals.," J Bone Joint Surg Am, vol. 64, no. 5, pp. 713-720, 1982.

[5] K. Sato, S. Kikuchi, and T. Yonezawa, "In vivo intradiscal pressure measurement in healthy individuals and in patients with ongoing back problems," Spine, vol. 24, no. 23, p. 2468, 1999.

[6] H.-J. Wilke, P. Neef, B. Hinz, H. Seidel, and L. Claes, "Intradiscal pressure together with anthropometric data-a data set for the validation of models," Clinical Biomechanics, vol. 16, pp. S111-S126, 2001.

[7] I. Takahashi, S.-i. Kikuchi, K. Sato, and N. Sato, "Mechanical load of the lumbar spine during forward bending motion of the trunk-a biomechanical study," Spine, vol. 31 , no. 1, pp. 18-23, 2006.

[8] D. J. Polga, B. P. Beaubien, P. M. Kallemeier, K. P. Schellhas, W. D. Lew, G. R. Buttermann, and K. B. Wood, "Measurement of in vivo intradiscal pressure in healthy thoracic intervertebral discs," Spine, vol. 29, no. 12, pp. 1320-1324, 2004.

[9] A. R. Meir, J. C. Fairbank, D. A. Jones, D. S. McNally, and J. P. Urban, "High pressures and asymmetrical stresses in the scoliotic disc in the absence of muscle loading," Scoliosis, vol. 2, no. 1, p. 4, 2007.

[10] A. Rohlmann, F. Graichen, R. Kayser, A. Bender, and G. Bergmann, "Loads on a telemeterized vertebral body replacement measured in two patients," Spine, vol. 33, no. 11, pp. 1170-1179, 2008.

[11] M. Dreischarf, L. Albiol, T. Zander, R. Arshad, F. Graichen, G. Bergmann, H. Schmidt, and A. Rohlmann, "In vivo implant forces acting on a vertebral body replacement 
during upper body flexion,” Journal of biomechanics, vol. 48, no. 4, pp. 560-565, 2015 .

[12] M. Dreischarf, A. Rohlmann, R. Zhu, H. Schmidt, and T. Zander, "Is it possible to estimate the compressive force in the lumbar spine from intradiscal pressure measurements? a finite element evaluation," Medical engineering \& physics, vol. 35, no. 9, pp. 1385-1390, 2013.

[13] J. Cholewicki and S. M. McGill, "Mechanical stability of the in vivo lumbar spine: implications for injury and chronic low back pain," Clinical biomechanics, vol. 11, no. 1, pp. 1-15, 1996.

[14] N. Farahpour, H. Younesian, and F. Bahrpeyma, "Electromyographic activity of erector spinae and external oblique muscles during trunk lateral bending and axial rotation in patients with adolescent idiopathic scoliosis and healthy subjects," Clinical Biomechanics, vol. 30, no. 5, pp. 411-417, 2015.

[15] D. Winter, A. Fuglevand, and S. Archer, "Crosstalk in surface electromyography: theoretical and practical estimates," Journal of Electromyography and Kinesiology, vol. 4 , no. 1, pp. 15-26, 1994.

[16] D. Gagnon, A. Plamondon, and C. Larivière, "A biomechanical comparison between expert and novice manual materials handlers using a multi-joint emg-assisted optimization musculoskeletal model of the lumbar spine," Journal of biomechanics, vol. 49, no. 13, pp. 2938-2945, 2016.

[17] L. Bresnahan, R. G. Fessler, and R. N. Natarajan, "Evaluation of change in muscle activity as a result of posterior lumbar spine surgery using a dynamic modeling system,” Spine, vol. 35, no. 16, pp. E761-E767, 2010.

[18] M. Wessels, J. J. Homminga, E. E. Hekman, and G. J. Verkerke, "A novel anchoring system for use in a nonfusion scoliosis correction device," The Spine Journal, vol. 14 , no. 11 , pp. 2740-2747, 2014.

[19] A. B. Schultz and J. O. Galante, "A mathematical model for the study of the mechanics of the human vertebral column," Journal of biomechanics, vol. 3, no. 4, pp. 405-416, 1970 .

[20] M. Panjabi and A. A. White III, "A mathematical approach for three-dimensional analysis of the mechanics of the spine," Journal of Biomechanics, vol. 4, no. 3, pp. 203-211, 1971.

[21] M. M. Panjabi, “Three-dimensional mathematical model of the human spine structure," Journal of Biomechanics, vol. 6, no. 6, pp. 671-680, 1973. 
[22] A. Schultz, T. Belytschko, T. Andriacchi, and J. Galante, "Analog studies of forces in the human spine: mechanical properties and motion segment behavior," Journal of Biomechanics, vol. 6, no. 4, pp. 373-383, 1973.

[23] T. Andriacchi, A. Schultz, T. Belytschko, and J. Galante, "A model for studies of mechanical interactions between the human spine and rib cage," Journal of biomechanics, vol. 7, no. 6, pp. 497-507, 1974.

[24] A. Erdemir, S. McLean, W. Herzog, and A. J. van den Bogert, "Model-based estimation of muscle forces exerted during movements," Clinical Biomechanics, vol. 22, no. 2, pp. 131-154, 2007.

[25] J. Rasmussen, M. Damsgaard, and M. Voigt, "Muscle recruitment by the $\mathrm{min} / \mathrm{max}$ criterion - a comparative numerical study," Journal of biomechanics, vol. 34, no. 3, pp. 409-415, 2001.

[26] M. De Zee, L. Hansen, C. Wong, J. Rasmussen, and E. B. Simonsen, "A generic detailed rigid-body lumbar spine model," Journal of biomechanics, vol. 40, no. 6, pp. 1219-1227, 2007.

[27] M. Christophy, N. A. F. Senan, J. C. Lotz, and O. M. O’Reilly, "A musculoskeletal model for the lumbar spine," Biomechanics and modeling in mechanobiology, vol. 11, no. 1-2, pp. 19-34, 2012.

[28] D. Gagnon, N. Arjmand, A. Plamondon, A. Shirazi-Adl, and C. Larivière, "An improved multi-joint emg-assisted optimization approach to estimate joint and muscle forces in a musculoskeletal model of the lumbar spine," Journal of biomechanics, vol. 44, no. 8, pp. 1521-1529, 2011.

[29] M. E. Raabe and A. M. Chaudhari, "An investigation of jogging biomechanics using the full-body lumbar spine model: Model development and validation," Journal of biomechanics, vol. 49, no. 7, pp. 1238-1243, 2016.

[30] F. Ghezelbash, Z. El Ouaaid, A. Shirazi-Adl, A. Plamondon, and N. Arjmand, "Trunk musculoskeletal response in maximum voluntary exertions: A combined measurement-modeling investigation," Journal of biomechanics, vol. 70, pp. 124 $133,2018$.

[31] A. Eskandari, N. Arjmand, A. Shirazi-Adl, and F. Farahmand, "Subject-specific 2d/3d image registration and kinematics-driven musculoskeletal model of the spine," Journal of Biomechanics, vol. 57, pp. 18-26, 2017.

[32] M. Akhavanfar, H. Kazemi, A. Eskandari, and N. Arjmand, "Obesity and spinal loads; a combined $\mathrm{mr}$ imaging and subject-specific modeling investigation," Journal of biomechanics, vol. 70, pp. 102-112, 2018. 
[33] A. G. Bruno, M. L. Bouxsein, and D. E. Anderson, "Development and validation of a musculoskeletal model of the fully articulated thoracolumbar spine and rib cage," Journal of biomechanical engineering, vol. 137, no. 8, p. 081003, 2015.

[34] D. Ignasiak, S. Dendorfer, and S. J. Ferguson, "Thoracolumbar spine model with articulated ribcage for the prediction of dynamic spinal loading," Journal of biomechanics, vol. 49, no. 6, pp. 959-966, 2016.

[35] A. N. Vasavada, S. Li, and S. L. Delp, "Influence of muscle morphometry and moment arms on the moment-generating capacity of human neck muscles," Spine, vol. 23, no. 4, pp. 412-422, 1998.

[36] K. J. Netto, A. F. Burnett, J. P. Green, and J. P. Rodrigues, "Validation of an emgdriven, graphically based isometric musculoskeletal model of the cervical spine," Journal of biomechanical engineering, vol. 130, no. 3, p. 031014, 2008.

[37] T. Khurelbaatar, K. Kim, and Y. H. Kim, "A cervico-thoraco-lumbar multibody dynamic model for the estimation of joint loads and muscle forces," Journal of biomechanical engineering, vol. 137, no. 11, p. 111001, 2015.

[38] J. Borst, P. A. Forbes, R. Happee, and D. H. Veeger, "Muscle parameters for musculoskeletal modelling of the human neck," Clinical Biomechanics, vol. 26, no. 4, pp. 343-351, 2011.

[39] V. Carbone, R. Fluit, P. Pellikaan, M. Van Der Krogt, D. Janssen, M. Damsgaard, L. Vigneron, T. Feilkas, H. Koopman, and N. Verdonschot, "Tlem 2.0-a comprehensive musculoskeletal geometry dataset for subject-specific modeling of lower extremity," Journal of biomechanics, vol. 48, no. 5, pp. 734-741, 2015.

[40] D. Ignasiak, S. J. Ferguson, and N. Arjmand, "A rigid thorax assumption affects model loading predictions at the upper but not lower lumbar levels," Journal of biomechanics, vol. 49, no. 13, pp. 3074-3078, 2016.

[41] D. E. Anderson, J. M. D’Agostino, A. G. Bruno, R. K. Manoharan, and M. L. Bouxsein, "Regressions for estimating muscle parameters in the thoracic and lumbar trunk for use in musculoskeletal modeling," Journal of biomechanics, vol. 45, no. 1, pp. 66-75, 2012.

[42] S. L. Delp, S. Suryanarayanan, W. M. Murray, J. Uhlir, and R. J. Triolo, "Architecture of the rectus abdominis, quadratus lumborum, and erector spinae," Journal of biomechanics, vol. 34, no. 3, pp. 371-375, 2001.

[43] V. Carbone, M. van der Krogt, H. F. Koopman, and N. Verdonschot, "Sensitivity of subject-specific models to hill muscle-tendon model parameters in simulations of gait," Journal of biomechanics, vol. 49, no. 9, pp. 1953-1960, 2016. 
[44] S. H. Brown and M. E. Gerling, "Importance of sarcomere length when determining muscle physiological cross-sectional area: a spine example," Proceedings of the Institution of Mechanical Engineers, Part H: Journal of Engineering in Medicine, vol. 226, no. 5, pp. 384-388, 2012.

[45] S. M. McGill, "A myoelectrically based dynamic three-dimensional model to predict loads on lumbar spine tissues during lateral bending," Journal of biomechanics, vol. 25 , no. 4, pp. 395-414, 1992.

[46] A. Abouhossein, B. Weisse, and S. J. Ferguson, "A multibody modelling approach to determine load sharing between passive elements of the lumbar spine," Computer methods in biomechanics and biomedical engineering, vol. 14, no. 06, pp. 527-537, 2011.

[47] T. Zander, M. Dreischarf, H. Schmidt, G. Bergmann, and A. Rohlmann, "Spinal loads as influenced by external loads: a combined in vivo and in silico investigation," Journal of biomechanics, vol. 48, no. 4, pp. 578-584, 2015.

[48] M. Putzer, I. Ehrlich, J. Rasmussen, N. Gebbeken, and S. Dendorfer, "Sensitivity of lumbar spine loading to anatomical parameters," Journal of biomechanics, vol. 49, no. 6, pp. 953-958, 2016.

[49] H.-J. Wilke, A. Kienle, S. Maile, V. Rasche, and N. Berger-Roscher, "A new dynamic six degrees of freedom disc-loading simulator allows to provoke disc damage and herniation," European Spine Journal, vol. 25, no. 5, pp. 1363-1372, 2016.

[50] S. S. Blemker, D. S. Asakawa, G. E. Gold, and S. L. Delp, "Image-based musculoskeletal modeling: Applications, advances, and future opportunities," Journal of magnetic resonance imaging, vol. 25, no. 2, pp. 441-451, 2007.

[51] V. Carbone, M. Van der Krogt, H. Koopman, and N. Verdonschot, "Sensitivity of subject-specific models to errors in musculo-skeletal geometry," Journal of biomechanics, vol. 45, no. 14, pp. 2476-2480, 2012.

[52] L. K. Kamibayashi and F. J. Richmond, "Morphometry of human neck muscles," Spine, vol. 23, no. 12 , pp. 1314-1323, 1998.

[53] N. Bogduk, J. E. Macintosh, and M. J. Pearcy, "A universal model of the lumbar back muscles in the upright position.," Spine, vol. 17, no. 8, pp. 897-913, 1992.

[54] N. Bogduk, M. Pearcy, and G. Hadfield, "Anatomy and biomechanics of psoas major," Clinical Biomechanics, vol. 7, no. 2, pp. 109-119, 1992.

[55] N. Bogduk, G. Johnson, and D. Spalding, "The morphology and biomechanics of latissimus dorsi," Clinical Biomechanics, vol. 13, no. 6, pp. 377-385, 1998. 
[56] J. E. Macintosh and N. Bogduk, "The attachments of the lumbar erector spinae.," Spine, vol. 16, no. 7, pp. 783-792, 1991.

[57] J. E. Macintosh, F. Valencia, N. Bogduk, and R. R. Munro, "The morphology of the human lumbar multifidus," Clinical Biomechanics, vol. 1, no. 4, pp. 196-204, 1986.

[58] S. Phillips, S. Mercer, and N. Bogduk, "Anatomy and biomechanics of quadratus lumborum," Proceedings of the Institution of Mechanical Engineers, Part H: Journal of Engineering in Medicine, vol. 222, no. 2, pp. 151-159, 2008.

[59] N. Arjmand, D. Gagnon, A. Plamondon, A. Shirazi-Adl, and C. Lariviere, "Comparison of trunk muscle forces and spinal loads estimated by two biomechanical models," Clinical Biomechanics, vol. 24, no. 7, pp. 533-541, 2009.

[60] M. K. Horsman, H. F. Koopman, F. Van der Helm, L. P. Prosé, and H. Veeger, "Morphological muscle and joint parameters for musculoskeletal modelling of the lower extremity," Clinical biomechanics, vol. 22, no. 2, pp. 239-247, 2007.

[61] M. D. K. Breteler, C. W. Spoor, and F. C. Van der Helm, "Measuring muscle and joint geometry parameters of a shoulder for modeling purposes," Journal of biomechanics, vol. 32, no. 11, pp. 1191-1197, 1999.

[62] J. E. Macintosh and N. Bogduk, "1987 volvo award in basic science: The morphology of the lumbar erector spinae.," Spine, vol. 12, no. 7, pp. 658-668, 1987.

[63] H. Cross, R. West, and T. Dutson, "Comparison of methods for measuring sarcomere length in beef semitendinosus muscle," Meat science, vol. 5, no. 4, pp. 261-266, 1981.

[64] S. H. Brown, S. R. Ward, M. S. Cook, and R. L. Lieber, "Architectural analysis of human abdominal wall muscles: implications for mechanical function," Spine, vol. 36 , no. 5 , p. $355,2011$.

[65] P. Besl andN and D. McKay, "A method for registration of 3-d shapes," IEEE Trans. Patt. Anal. Mach. Intell, pp. 14-2, 1992.

[66] P. Pellikaan, M. van der Krogt, V. Carbone, R. Fluit, L. Vigneron, J. Van Deun, N. Verdonschot, and H. Koopman, "Evaluation of a morphing based method to estimate muscle attachment sites of the lower extremity," Journal of biomechanics, vol. 47, no. 5, pp. 1144-1150, 2014.

[67] F. C. Van der Helm, H. Veeger, G. Pronk, L. Van der Woude, and R. Rozendal, "Geometry parameters for musculoskeletal modelling of the shoulder system," Journal of biomechanics, vol. 25, no. 2, pp. 129-144, 1992. 
[68] M. E. Gerling and S. H. Brown, "Architectural analysis and predicted functional capability of the human latissimus dorsi muscle," Journal of anatomy, vol. 223, no. 2, pp. 112-122, 2013.

[69] G. J. Regev, C. W. Kim, A. Tomiya, Y. P. Lee, H. Ghofrani, S. R. Garfin, R. L. Lieber, and S. R. Ward, "Psoas muscle architectural design, in vivo sarcomere length range, and passive tensile properties support its role as a lumbar spine stabilizer," Spine, vol. 36, no. 26, pp. E1666-E1674, 2011.

[70] H. Veeger, F. Van der Helm, L. Van Der Woude, G. Pronk, and R. Rozendal, "Inertia and muscle contraction parameters for musculoskeletal modelling of the shoulder mechanism,” Journal of biomechanics, vol. 24, no. 7, pp. 615-629, 1991.

[71] S. R. Ward, A. Tomiya, G. J. Regev, B. E. Thacker, R. C. Benzl, C. W. Kim, and R. L. Lieber, "Passive mechanical properties of the lumbar multifidus muscle support its role as a stabilizer," Journal of biomechanics, vol. 42, no. 10, pp. 1384-1389, 2009 .

[72] S. R. Ward, C. M. Eng, L. H. Smallwood, and R. L. Lieber, "Are current measurements of lower extremity muscle architecture accurate?," Clinical orthopaedics and related research, vol. 467, no. 4, pp. 1074-1082, 2009.

[73] S. R. Ward, C. W. Kim, C. M. Eng, L. J. Gottschalk IV, A. Tomiya, S. R. Garfin, and R. L. Lieber, "Architectural analysis and intraoperative measurements demonstrate the unique design of the multifidus muscle for lumbar spine stability," The Journal of Bone and Joint Surgery. American volume., vol. 91, no. 1, p. 176, 2009.

[74] F. E. Zajac, "Muscle and tendon properties models scaling and application to biomechanics and motor," Critical reviews in biomedical engineering, vol. 17, no. 4, pp. 359-411, 1989.

[75] T. T. Dao and M.-C. H. B. Tho, "Assessment of parameter uncertainty in rigid musculoskeletal simulation using a probabilistic approach," Journal of Musculoskeletal Research, vol. 18, no. 03, p. 1550013, 2015.

[76] V. Carbone, R. Fluit, P. Pellikaan, M. Van Der Krogt, D. Janssen, M. Damsgaard, L. Vigneron, T. Feilkas, H. F. Koopman, and N. Verdonschot, "Tlem 2.0-a comprehensive musculoskeletal geometry dataset for subject-specific modeling of lower extremity," Journal of biomechanics, vol. 48, no. 5, pp. 734-741, 2015.

[77] F. Bustami, "A new description of the lumbar erector spinae muscle in man.," Journal of anatomy, vol. 144, p. 81, 1986. 
[78] N. K. Mahato, "Pars inter-articularis and laminar morphology of the terminal lumbar vertebra in lumbosacral transitional variations," North American journal of medical sciences, vol. 5, no. 6, p. 357, 2013.

[79] A. Cutts, "Shrinkage of muscle fibres during the fixation of cadaveric tissue.," Journal of anatomy, vol. 160, p. 75, 1988.

[80] F. C. Van der Helm and R. Veenbaas, "Modelling the mechanical effect of muscles with large attachment sites: application to the shoulder mechanism," Journal of biomechanics, vol. 24, no. 12, pp. 1151-1163, 1991.

[81] S. M. Walker and G. R. Schrodt, "I segment lengths and thin filament periods in skeletal muscle fibers of the rhesus monkey and the human," The Anatomical Record, vol. 178, no. 1, pp. 63-81, 1974.

[82] B. W. Infantolino, M. J. Ellis, and J. H. Challis, "Individual sarcomere lengths in whole muscle fibers and optimal fiber length computation," The Anatomical Record, vol. 293, no. 11, pp. 1913-1919, 2010.

[83] J. Langenderfer, S. A. Jerabek, V. B. Thangamani, J. E. Kuhn, and R. E. Hughes, "Musculoskeletal parameters of muscles crossing the shoulder and elbow and the effect of sarcomere length sample size on estimation of optimal muscle length," Clinical Biomechanics, vol. 19, no. 7, pp. 664-670, 2004.

[84] R. Bayoglu, L. Geeraedts, K. H. Groenen, N. Verdonschot, B. Koopman, and J. Homminga, "Twente spine model: A complete and coherent dataset for musculo-skeletal modeling of the lumbar region of the human spine," Journal of biomechanics, vol. 53, pp. 111-119, 2017.

[85] R. Arshad, T. Zander, M. Dreischarf, and H. Schmidt, "Influence of lumbar spine rhythms and intra-abdominal pressure on spinal loads and trunk muscle forces during upper body inclination," Medical Engineering and Physics, vol. 38, no. 4, pp. 333-338, 2016.

[86] A. M. Briggs, J. H. Van Dieën, T. V. Wrigley, A. M. Greig, B. Phillips, S. K. Lo, and K. L. Bennell, "Thoracic kyphosis affects spinal loads and trunk muscle force," Physical therapy, vol. 87, no. 5, pp. 595-607, 2007.

[87] K.-S. Han, A. Rohlmann, T. Zander, and W. R. Taylor, "Lumbar spinal loads vary with body height and weight," Medical Engineering and Physics, vol. 35, no. 7, pp. 969-977, 2013.

[88] E. M. Arnold, S. R. Ward, R. L. Lieber, and S. L. Delp, "A model of the lower limb for analysis of human movement," Annals of biomedical engineering, vol. 38, no. 2, pp. 269-279, 2010. 
[89] G. Valente, L. Pitto, D. Testi, A. Seth, S. L. Delp, R. Stagni, M. Viceconti, and F. Taddei, "Are subject-specific musculoskeletal models robust to the uncertainties in parameter identification?," PLoS One, vol. 9, no. 11, p. e112625, 2014.

[90] L. Modenese, E. Ceseracciu, M. Reggiani, and D. G. Lloyd, "Estimation of musculotendon parameters for scaled and subject specific musculoskeletal models using an optimization technique," Journal of biomechanics, vol. 49, no. 2, pp. 141-148, 2016.

[91] D. G. Thelen, "Adjustment of muscle mechanics model parameters to simulate dynamic contractions in older adults," Journal of biomechanical engineering, vol. 125, no. 1, pp. 70-77, 2003.

[92] M. Fidler and R. Jowett, "Muscle imbalance in the aetiology of scoliosis," Bone \& Joint Journal, vol. 58, no. 2, pp. 200-201, 1976.

[93] R. Bayoglu, L. Geeraedts, K. Groenen, N. Verdonschot, B. Koopman, and J. Homminga, "How valid are left-right symmetrical musculo-skeletal models?," Proceedings of the $22^{\text {nd }}$ Congress of the European Society of Biomechanics; 2016 July 10-13; Lyon, France., 2016.

[94] S. V. Brooks and J. A. Faulkner, "Skeletal muscle weakness in old age: underlying mechanisms.," Medicine and science in sports and exercise, vol. 26, no. 4, pp. 432439, 1994.

[95] K. Häkkinen and A. Häkkinen, "Muscle cross-sectional area, force production and relaxation characteristics in women at different ages," European journal of applied physiology and occupational physiology, vol. 62, no. 6, pp. 410-414, 1991.

[96] J. J. Morrow and W. Hosler, "Strength comparisons in untrained men and trained women athletes.," Medicine and science in sports and exercise, vol. 13, no. 3, pp. 194-197, 1981.

[97] D. A. Lewis, E. Kamon, and J. L. Hodgson, "Physiological differences between genders implications for sports conditioning," Sports medicine, vol. 3, no. 5, pp. 357-369, 1986.

[98] W. R. Frontera, V. A. Hughes, K. J. Lutz, and W. J. Evans, "A cross-sectional study of muscle strength and mass in 45-to 78-yr-old men and women," Journal of applied physiology, vol. 71, no. 2, pp. 644-650, 1991.

[99] J.-H. Kim, H.-S. Won, I.-H. Chung, and I.-B. Kim, “The enigmatic subcostal muscle: Anatomical study with application to spine and chest pain syndromes and avoidance of confusion on imaging," Clinical Anatomy, vol. 28, no. 8, pp. 1017-1021, 2015 . 
[100] H. Gray, R. Warwick, and P. L. Williams, Gray's anatomy, 35th edition. Longman, 1973.

[101] T. Bojadsen, E. Silva, A. Rodrigues, and A. Amadio, "Comparative study of mm. multifidi in lumbar and thoracic spine," Journal of Electromyography and Kinesiology, vol. 10 , no. 3 , pp. 143-149, 2000.

[102] J. Cornwall, M. D. Stringer, and M. Duxson, "Functional morphology of the thoracolumbar transversospinal muscles," Spine, vol. 36, no. 16, pp. E1053-E1061, 2011.

[103] W. Langenberg and S. Jüschke, "Morphologie und innervation der mm. levatores costarum und ihre beziehung zu den $\mathrm{mm}$. intertransversarii laterales lumborum. untersuchungen an mensch und känguruh," Zeitschrift für Anatomie und Entwicklungsgeschichte, vol. 130, no. 3, pp. 255-264, 1970.

[104] G. J. Meijer, J. Homminga, E. E. Hekman, A. Veldhuizen, and G. J. Verkerke, “The effect of three-dimensional geometrical changes during adolescent growth on the biomechanics of a spinal motion segment," Journal of biomechanics, vol. 43, no. 8, pp. 1590-1597, 2010.

[105] R. Bayoglu, L. Geeraedts, K. H. Groenen, N. Verdonschot, B. Koopman, and J. Homminga, "Twente spine model: A complete and coherent dataset for musculo-skeletal modeling of the thoracic and cervical regions of the human spine," Journal of biomechanics, vol. 58, pp. 52-63, 2017.

[106] D. J. Pearsall, J. G. Reid, and L. A. Livingston, "Segmental inertial parameters of the human trunk as determined from computed tomography," Annals of biomedical engineering, vol. 24, no. 2, pp. 198-210, 1996.

[107] M. K. J. de Jager, Mathematical head-neck models for acceleration impacts. CopyPrint, 2000.

[108] P. De Leva, “Adjustments to zatsiorsky-seluyanov's segment inertia parameters," Journal of biomechanics, vol. 29, no. 9, pp. 1223-1230, 1996.

[109] N. Arjmand, D. Gagnon, A. Plamondon, A. Shirazi-Adl, and C. Lariviere, "A comparative study of two trunk biomechanical models under symmetric and asymmetric loadings," Journal of biomechanics, vol. 43, no. 3, pp. 485-491, 2010.

[110] I. A. Stokes, "Three-dimensional terminology of spinal deformity: A report presented to the scoliosis research society by the scoliosis research society working group on 3-d terminology of spinal deformity.," Spine, vol. 19, no. 2, pp. 236-248, 1994.

[111] M. J. Pearcy and N. Bogduk, "Instantaneous axes of rotation of the lumbar intervertebral joints.," Spine, vol. 13, no. 9, pp. 1033-1041, 1988. 
[112] J. Dvorak, M. Panjabi, J. Novotny, and J. Antinnes, "In vivo flexion/extension of the normal cervical spine," Journal of Orthopaedic Research, vol. 9, no. 6, pp. 828-834, 1991.

[113] P. Minotti and C. Lexcellent, "Geometric and kinematic modelling of a human costal slice,” Journal of biomechanics, vol. 24, no. 3-4, pp. 213-221, 1991.

[114] A. B. Schultz, D. R. Benson, and C. Hirsch, "Force-deformation properties of human costo-sternal and costo-vertebral articulations," Journal of biomechanics, vol. 7, no. 3, pp. 311IN23313-312IN27318, 1974.

[115] S. Duprey, D. Subit, H. Guillemot, and R. W. Kent, "Biomechanical properties of the costovertebral joint," Medical engineering \& physics, vol. 32, no. 2, pp. 222-227, 2010.

[116] M. S. Andersen, M. Damsgaard, and J. Rasmussen, "Kinematic analysis of overdeterminate biomechanical systems," Computer Methods in Biomechanics and Biomedical Engineering, vol. 12, no. 4, pp. 371-384, 2009.

[117] J. A. Ashton-Miller and A. B. Schultz, "Biomechanics of the human spine and trunk.," Exercise and sport sciences reviews, vol. 16, no. 1, pp. 169-204, 1988.

[118] S. P. Moroney, A. B. Schultz, J. A. Miller, and G. B. Andersson, "Load-displacement properties of lower cervical spine motion segments," Journal of biomechanics, vol. 21, no. 9, pp. 769-779, 1988.

[119] M. M. Panjabi, R. A. Brand, and A. A. White, "Three-dimensional flexibility and stiffness properties of the human thoracic spine," Journal of Biomechanics, vol. 9, no. 4, pp. 185-192, 1976.

[120] T. A. Schmidt, H. S. An, T.-H. Lim, B. H. Nowicki, and V. M. Haughton, "The stiffness of lumbar spinal motion segments with a high-intensity zone in the anulus fibrosus," Spine, vol. 23, no. 20, pp. 2167-2173, 1998.

[121] I. White, A.A. and M. Panjabi, "The basic kinematics of the human spine: A review of past and current knowledge.," Spine, vol. 3, no. 1, pp. 12-20, 1978.

[122] K. W. Wong, K. D. Luk, J. C. Leong, S. F. Wong, and K. K. Wong, "Continuous dynamic spinal motion analysis," Spine, vol. 31, no. 4, pp. 414-419, 2006.

[123] R. Fujii, H. Sakaura, Y. Mukai, N. Hosono, T. Ishii, M. Iwasaki, H. Yoshikawa, and K. Sugamoto, "Kinematics of the lumbar spine in trunk rotation: in vivo threedimensional analysis using magnetic resonance imaging," European spine journal, vol. 16 , no. 11 , pp. $1867-1874,2007$. 
[124] A. Rozumalski, M. H. Schwartz, R. Wervey, A. Swanson, D. C. Dykes, and T. Novacheck, "The in vivo three-dimensional motion of the human lumbar spine during gait," Gait \& posture, vol. 28, no. 3, pp. 378-384, 2008.

[125] T. Fujimori, M. Iwasaki, Y. Nagamoto, T. Ishii, M. Kashii, T. Murase, T. Sugiura, Y. Matsuo, K. Sugamoto, and H. Yoshikawa, "Kinematics of the thoracic spine in trunk rotation: in vivo 3-dimensional analysis," Spine, vol. 37, no. 21, pp. E1318E1328, 2012.

[126] T. Fujimori, M. Iwasaki, Y. Nagamoto, Y. Matsuo, T. Ishii, T. Sugiura, M. Kashii, T. Murase, K. Sugamoto, and H. Yoshikawa, "Kinematics of the thoracic spine in trunk lateral bending: in vivo three-dimensional analysis," The Spine Journal, vol. 14, no. 9, pp. 1991-1999, 2014.

[127] A. Tafazzol, N. Arjmand, A. Shirazi-Adl, and M. Parnianpour, "Lumbopelvic rhythm during forward and backward sagittal trunk rotations: combined in vivo measurement with inertial tracking device and biomechanical modeling," Clinical Biomechanics, vol. 29, no. 1, pp. 7-13, 2014.

[128] K.-S. Han, T. Zander, W. R. Taylor, and A. Rohlmann, "An enhanced and validated generic thoraco-lumbar spine model for prediction of muscle forces," Medical engineering \& physics, vol. 34, no. 6, pp. 709-716, 2012.

[129] J. Homminga, A. M. Lehr, G. J. Meijer, M. M. Janssen, T. P. Schlösser, G. J. Verkerke, and R. M. Castelein, "Posteriorly directed shear loads and disc degeneration affect the torsional stiffness of spinal motion segments: a biomechanical modeling study," Spine, vol. 38, no. 21, pp. E1313-E1319, 2013.

[130] S. Iyer, B. A. Christiansen, B. J. Roberts, M. J. Valentine, R. K. Manoharan, and M. L. Bouxsein, "A biomechanical model for estimating loads on thoracic and lumbar vertebrae," Clinical biomechanics, vol. 25, no. 9, pp. 853-858, 2010.

[131] M. M. Panjabi, K. Takata, V. Goel, D. Federico, T. Oxland, J. Duranceau, and M. Krag, "Thoracic human vertebrae quantitative three-dimensional anatomy.," Spine, vol. 16 , no. 8, pp. 888-901, 1991.

[132] C. J. Snijders, G. H. Van Dijke, and E. Roosch, "A biomechanical model for the analysis of the cervical spine in static postures," Journal of Biomechanics, vol. 24, no. 9, pp. 783-792, 1991.

[133] X. Meng, A. G. Bruno, B. Cheng, W. Wang, M. L. Bouxsein, and D. E. Anderson, "Incorporating six degree-of-freedom intervertebral joint stiffness in a lumbar spine musculoskeletal model - method and performance in flexed postures," Journal of biomechanical engineering, vol. 137, no. 10, p. 101008, 2015. 
[134] M. Senteler, A. Aiyangar, B. Weisse, M. Farshad, and J. G. Snedeker, "Sensitivity of intervertebral joint forces to center of rotation location and trends along its migration path," Journal of biomechanics, vol. 70, pp. 140-148, 2018.

[135] F. Ghezelbash, A. Eskandari, A. Shirazi-Adl, N. Arjmand, Z. El-Ouaaid, and A. Plamondon, "Effects of motion segment simulation and joint positioning on spinal loads in trunk musculoskeletal models," Journal of biomechanics, 2017.

[136] A. G. Bruno, H. Mokhtarzadeh, B. T. Allaire, K. R. Velie, M. C. De Paolis Kaluza, D. E. Anderson, and M. L. Bouxsein, "Incorporation of ct-based measurements of trunk anatomy into subject-specific musculoskeletal models of the spine influences vertebral loading predictions," Journal of Orthopaedic Research, vol. 35, no. 10, pp. 2164-2173, 2017.

[137] R. Bayoglu, P. E. Galibarov, N. Verdonschot, B. Koopman, and J. Homminga, "Twente spine model: A thorough investigation of the spinal loads in a complete and coherent musculoskeletal model of the human spine," Medical Engineering \& Physics, 2019.

[138] T. Zander, M. Dreischarf, and H. Schmidt, "Sensitivity analysis of the position of the intervertebral centres of reaction in upright standing-a musculoskeletal model investigation of the lumbar spine," Medical engineering $\&$ physics, vol. 38, no. 3, pp. 297-301, 2016.

[139] M. Putzer, S. Auer, W. Malpica, F. Suess, and S. Dendorfer, "A numerical study to determine the effect of ligament stiffness on kinematics of the lumbar spine during flexion," BMC musculoskeletal disorders, vol. 17, no. 1, p. 95, 2016.

[140] M. A. Nussbaum, D. B. Chaffin, and C. J. Rechtien, "Muscle lines-of-action affect predicted forces in optimization-based spine muscle modeling," Journal of Biomechanics, vol. 28, no. 4, pp. 401-409, 1995.

[141] L. Hansen, M. De Zee, J. Rasmussen, T. B. Andersen, C. Wong, and E. B. Simonsen, "Anatomy and biomechanics of the back muscles in the lumbar spine with reference to biomechanical modeling," Spine, vol. 31, no. 17, pp. 1888-1899, 2006.

[142] J. Rasmussen, M. Damsgaard, and M. Voigt, "Muscle recruitment by the $\mathrm{min} / \mathrm{max}$ criterion-a comparative numerical study," Journal of biomechanics, vol. 34, no. 3, pp. 409-415, 2001.

[143] M. Senteler, B. Weisse, D. A. Rothenfluh, and J. G. Snedeker, "Intervertebral reaction force prediction using an enhanced assembly of opensim models," Computer methods in biomechanics and biomedical engineering, vol. 19, no. 5, pp. 538-548, 2016. 
[144] C. Redl, M. Gfoehler, and M. G. Pandy, "Sensitivity of muscle force estimates to variations in muscle-tendon properties," Human movement science, vol. 26, no. 2, pp. 306-319, 2007.

[145] M. E. Lund, J. Rasmussen, and M. Andersen, "Anypytools: A python package for reproducible research with the anybody modeling system," The Journal of Open Source Software, vol. 4, p. 1108, 2019.

[146] F. Ghezelbash, N. Arjmand, and A. Shirazi-Adl, "Effect of intervertebral translational flexibilities on estimations of trunk muscle forces, kinematics, loads, and stability," Computer methods in biomechanics and biomedical engineering, vol. 18, no. 16, pp. 1760-1767, 2015.

[147] M. E. Lund, M. de Zee, M. S. Andersen, and J. Rasmussen, "On validation of multibody musculoskeletal models," Proceedings of the Institution of Mechanical Engineers, Part H: Journal of Engineering in Medicine, vol. 226, no. 2, pp. 82-94, 2012.

[148] J. A. Actis, J. D. Honegger, D. H. Gates, A. J. Petrella, L. A. Nolasco, and A. K. Silverman, "Validation of lumbar spine loading from a musculoskeletal model including the lower limbs and lumbar spine," Journal of biomechanics, vol. 68, pp. 107-114, 2018.

[149] K. W. Wong, J. C. Leong, M.-k. Chan, K. D. Luk, and W. W. Lu, "The flexion-extension profile of lumbar spine in 100 healthy volunteers," Spine, vol. 29, no. 15, pp. 1636$1641,2004$.

[150] W. J. Anderst, W. F. Donaldson, J. Y. Lee, and J. D. Kang, "Continuous cervical spine kinematics during in vivo dynamic flexion-extension," The Spine Journal, vol. 14, no. 7 , pp. 1221-1227, 2014.

[151] W. J. Anderst, W. F. Donaldson, J. Y. Lee, and J. D. Kang, “Three-dimensional intervertebral kinematics in the healthy young adult cervical spine during dynamic functional loading," Journal of biomechanics, vol. 48, no. 7, pp. 1286-1293, 2015.

[152] E. Gercek, F. Hartmann, S. Kuhn, J. Degreif, P. M. Rommens, and L. Rudig, "Dynamic angular three-dimensional measurement of multisegmental thoracolumbar motion in vivo," Spine, vol. 33, no. 21, pp. 2326-2333, 2008.

[153] R. A. Preuss and M. R. Popovic, "Three-dimensional spine kinematics during multidirectional, target-directed trunk movement in sitting," Journal of Electromyography and Kinesiology, vol. 20, no. 5, pp. 823-832, 2010.

[154] N.-G. Lee, J.-A. Lee, and J.-B. Kim, "A comparison of three-dimensional spine kinematics during multidirectional trunk movement between elderly subjects with 
degenerative spine disease and healthy young adults," Journal of Physical Therapy Science, vol. 25, no. 1, pp. 21-26, 2013.

[155] M. Narimani and N. Arjmand, "Three-dimensional primary and coupled range of motions and movement coordination of the pelvis, lumbar and thoracic spine in standing posture using inertial tracking device," Journal of biomechanics, vol. 69, pp. $169-174,2018$.

[156] P. Watson, C. Booker, C. Main, and A. Chen, "Surface electromyography in the identification of chronic low back pain patients: the development of the flexion relaxation ratio," Clinical Biomechanics, vol. 12, no. 3, pp. 165-171, 1997.

[157] J. K.-F. Ng, C. A. Richardson, M. Parnianpour, and V. Kippers, "Emg activity of trunk muscles and torque output during isometric axial rotation exertion: a comparison between back pain patients and matched controls," Journal of Orthopaedic Research, vol. 20, no. 1, pp. 112-121, 2002.

[158] H. J. Hermens, B. Freriks, R. Merletti, D. Stegeman, J. Blok, G. Rau, C. DisselhorstKlug, and G. Hägg, "European recommendations for surface electromyography," Roessingh research and development, vol. 8, no. 2, pp. 13-54, 1999.

[159] P. Konrad, "The abc of emg," A practical introduction to kinesiological electromyography, vol. 1, pp. 30-35, 2005.

[160] G. D. Cramer and S. A. Darby, Clinical Anatomy of the Spine, Spinal Cord, and ANS-E-Book.

Elsevier Health Sciences, 2017.

[161] C. Goodvin, E. J. Park, K. Huang, and K. Sakaki, "Development of a real-time threedimensional spinal motion measurement system for clinical practice," Medical and Biological Engineering and Computing, vol. 44, no. 12, pp. 1061-1075, 2006.

[162] J. M. Morris, G. Benner, and D. B. Lucas, "An electromyographic study of the intrinsic muscles of the back in man," Journal of anatomy, vol. 96, no. Pt 4, p. 509, 1962.

[163] C. Snijders, M. Bakker, A. Vleeming, R. Stoeckart, and H. Stam, "Oblique abdominal muscle activity in standing and in sitting on hard and soft seats," Clinical Biomechanics, vol. 10, no. 2, pp. 73-78, 1995.

[164] P. B. O’sullivan, K. M. Grahamslaw, M. Kendell, S. C. Lapenskie, N. E. Möller, and K. V. Richards, “The effect of different standing and sitting postures on trunk muscle activity in a pain-free population," Spine, vol. 27, no. 11, pp. 1238-1244, 2002.

[165] D. Farina, R. Merletti, and R. M. Enoka, "The extraction of neural strategies from the surface emg," Journal of applied physiology, vol. 96, no. 4, pp. 1486-1495, 2004. 
[166] D. Bakar, J. E. Tanenbaum, K. Phan, V. J. Alentado, M. P. Steinmetz, E. C. Benzel, and T. E. Mroz, "Decompression surgery for spinal metastases: a systematic review," Neurosurgical focus, vol. 41, no. 2, p. E2, 2016.

[167] R. Harel and L. Angelov, "Spine metastases: current treatments and future directions," European Journal of Cancer, vol. 46, no. 15, pp. 2696-2707, 2010.

[168] P. Sutcliffe, M. Connock, D. Shyangdan, R. Court, N. Kandala, and A. Clarke, "A systematic review of evidence on malignant spinal metastases: natural history and technologies for identifying patients at high risk of vertebral fracture and spinal cord compression.," Health Technology Assessment (Winchester, England), vol. 17 , no. 42 , p. $1,2013$.

[169] C. G. Fisher, C. P. DiPaola, T. C. Ryken, M. H. Bilsky, C. I. Shaffrey, S. H. Berven, J. S. Harrop, M. G. Fehlings, S. Boriani, D. Chou, et al., "A novel classification system for spinal instability in neoplastic disease: an evidence-based approach and expert consensus from the spine oncology study group," Spine, vol. 35, no. 22, pp. E1221-E1229, 2010.

[170] R. P. Crawford, C. E. Cann, and T. M. Keaveny, "Finite element models predict in vitro vertebral body compressive strength better than quantitative computed tomography," Bone, vol. 33, no. 4, pp. 744-750, 2003.

[171] K. Imai, I. Ohnishi, M. Bessho, and K. Nakamura, "Nonlinear finite element model predicts vertebral bone strength and fracture site," Spine, vol. 31, no. 16, pp. 1789 $1794,2006$.

[172] M. A. Liebschner, D. L. Kopperdahl, W. S. Rosenberg, and T. M. Keaveny, "Finite element modeling of the human thoracolumbar spine," Spine, vol. 28, no. 6, pp. 559$565,2003$.

[173] J. M. Buckley, K. Loo, and J. Motherway, "Comparison of quantitative computed tomography-based measures in predicting vertebral compressive strength," Bone, vol. 40, no. 3, pp. 767-774, 2007.

[174] Y. Chevalier, M. Charlebois, D. Pahr, P. Varga, P. Heini, E. Schneider, and P. Zysset, "A patient-specific finite element methodology to predict damage accumulation in vertebral bodies under axial compression, sagittal flexion and combined loads," Computer methods in biomechanics and biomedical engineering, vol. 11, no. 5, pp. 477-487, 2008.

[175] E. Dall'Ara, D. Pahr, P. Varga, F. Kainberger, and P. Zysset, “Qct-based finite element models predict human vertebral strength in vitro significantly better than simulated dexa," Osteoporosis International, vol. 23, no. 2, pp. 563-572, 2012. 
[176] C. E. Tschirhart, J. A. Finkelstein, and C. M. Whyne, "Metastatic burst fracture risk assessment based on complex loading of the thoracic spine," Annals of biomedical engineering, vol. 34, no. 3, pp. 494-505, 2006.

[177] K. H. Groenen, T. Bitter, T. C. van Veluwen, Y. M. van der Linden, N. Verdonschot, E. Tanck, and D. Janssen, "Case-specific non-linear finite element models to predict failure behavior in two functional spinal units," Journal of Orthopaedic Research ${ }^{\circledR}$, vol. 36, no. 12, pp. 3208-3218, 2018.

[178] M. Dreischarf, T. Zander, A. Shirazi-Adl, C. Puttlitz, C. Adam, C. Chen, V. Goel, A. Kiapour, Y. Kim, K. Labus, et al., "Comparison of eight published static finite element models of the intact lumbar spine: predictive power of models improves when combined together," Journal of biomechanics, vol. 47, no. 8, pp. 1757-1766, 2014 .

[179] S. A. Shirazi-Adl, S. C. Shrivastava, and A. M. Ahmed, "Stress analysis of the lumbar disc-body unit in compression. a three-dimensional nonlinear finite element study.," Spine, vol. 9, no. 2, pp. 120-134, 1984.

[180] J. H. Keyak, T. S. Kaneko, J. Tehranzadeh, and H. B. Skinner, "Predicting proximal femoral strength using structural engineering models.," Clinical orthopaedics and related research, vol. 437, pp. 219-228, 2005.

[181] J. Chazal, A. Tanguy, M. Bourges, G. Gaurel, G. Escande, M. Guillot, and G. Vanneuville, "Biomechanical properties of spinal ligaments and a histological study of the supraspinal ligament in traction," Journal of biomechanics, vol. 18, no. 3, pp. 167-176, 1985.

[182] F. A. Pintar, N. Yoganandan, T. Myers, A. Elhagediab, and A. Sances Jr, "Biomechanical properties of human lumbar spine ligaments," Journal of biomechanics, vol. 25, no. 11, pp. 1351-1356, 1992.

[183] B. Beyer, V. Sholukha, P. M. Dugailly, M. Rooze, F. Moiseev, V. Feipel, and S. V. S. Jan, "In vivo thorax $3 \mathrm{~d}$ modelling from costovertebral joint complex kinematics," Clinical Biomechanics, vol. 29, no. 4, pp. 434-438, 2014.

[184] B. Beyer, V. Feipel, V. Sholukha, L. Chèze, and S. V. S. Jan, "In-vivo analysis of sternal angle, sternal and sternocostal kinematics in supine humans during breathing," Journal of Biomechanics, 2017. 



\section{ACKNOWLEDGMENTS}

The work produced in this dissertation would have not been possible without the help, support, and kindness of several people who crossed my life in one way or another. I like to start my acknowledgments by thanking my co-supervisor Jasper Homminga. We have met in the first week of my Erasmus internship to discuss the possibility of a research assignment. I enjoyed our meetings a lot, which most of the time lasted more than an hour but were very fruitful. I think you have an extraordinary talent when you explain things! I really appreciate your enthusiasm for research and constant support when things did not go as planned. Sometimes you gave an extra hand for my work. It was very supportive and fun to see you coming to Nijmegen and drilling vertebrae in the anatomy lab! I also enjoyed our trip to Zurich and, of course, the beers on the way. I am pretty confident to say that you have been my role model both as an academician and a kind and knowledgeable person. So, Jasper, thanks a lot for everything!

I would like to gratefully thank my supervisors Bart Koopman and Nico Verdonschot. I always believed that I was very lucky to research under your guidance. You both are very knowledgeable and kind. Although you both were extremely busy and that it was often challenging to arrange a meeting where we all participate, you showed great enthusiasm and support for my research. I appreciated your attitude of always going further when things were not ideal. You contributed a lot to the progress of my research and my professional development. It was also very nice to enjoy the good talks, food, and beers outside the workplace, for example in Enschede, Nijmegen, and Brisbane! Nico and Bart, thanks a lot for everything!

Furthermore, my special thanks go to Leo Geeraedts. Without your great knowledge and experience in anatomy, the first two chapters of this book would be difficult to achieve or impossible. I really appreciated that you have spent five full weeks with me at the anatomy lab in Nijmegen and showed a big portion of enthusiasm and patience when things were difficult and complicated. Thanks a lot, Leo!

Finally, I would like to thank several colleagues, staff members, and students at the Biomechanical Engineering Group at the University of Twente and at the Orthopedic Research Laboratory in Nijmegen for their kindness, hospitability, help, and friendship. 



\section{BIOGRAPHY}

Riza Bayoglu was born on November $9^{t h}, 1986$, in Istanbul, Turkey. He studied Mechanical Engineering for a bachelor's degree at the Yeditepe University with an undergraduate scholarship from the Turkish Higher Education Council. In 2010, he graduated with first-class honors.

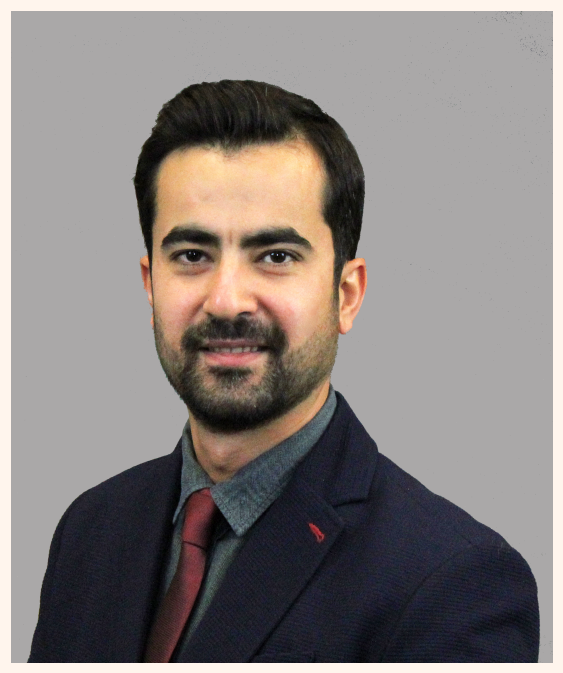

Subsequently, he was appointed to work as a research and teaching scholar in the same research group. He carried out these duties along with his master studies in Mechanical Engineering, during which he developed his first computational models to investigate the structural integrity of intact and implanted femurs by finite element analysis. The title of his master thesis was: A Physiologically Accurate Mechanical Representation of A Retrograde Nail-Bone Construct Under Walking Load, and this thesis was supervised by Dr. A. Fethi Okyar. He completed his master's studies with high honors.

After following a three-months Erasmus internship at the Department of Biomechanical Engineering at the University of Twente, he started to work as a Ph.D. researcher at the same group in 2014. His doctoral research was supervised by Dr. Bart Koopman, Dr. Nico Verdonschot, and Dr. Jasper Homminga. The main objective of his dissertation was to develop a validated-complete and coherent musculoskeletal model of the entire human spine and to apply this model in a clinical setting. During his Ph.D. study, he also organized departmental seminars and supervised several bachelor and master students.

He is currently a Post-Doctoral Researcher at the University of Denver in Colorado and is investigating healthy and implanted hip and knee biomechanics by computational and experimental methods. 

PUBLICATIONS

\section{Journal publications}

- Bayoglu, R., Geeraedts, L., Groenen, K.H.J., Verdonschot, N., Koopman, B., Homminga, J., Twente spine model: A complete and coherent dataset for musculo-skeletal modeling of the lumbar region of the human spine, Journal of Biomechanics, 2017, 53, 111-119.

- Bayoglu, R., Geeraedts, L., Groenen, K.H.J., Verdonschot, N., Koopman, B., Homminga, J., Twente spine model: A complete and coherent dataset for musculo-skeletal modeling of the thoracic and cervical regions of the human spine, Journal of Biomechanics, 2017, 58, 52-63.

- Bayoglu, R., Galibarov E.P., Verdonschot, N., Koopman, B., Homminga, J., Twente Spine Model: A thorough investigation of the spinal loads in a complete and coherent musculoskeletal model of the human spine, Medical Engineering \& Physics, 2019.

- Bayoglu, R., Guldeniz, O., Verdonschot, N., Koopman, B., Homminga, J., Sensitivity of muscle and intervertebral disc force computations to variations in muscle attachment sites. Under review.

- Bayoglu, R., Noort, G., Verdonschot, N., Koopman, B., Homminga, J., A coupled set of kinematic and electromyographic data for validation of spinal musculoskeletal models. To be submitted. 


\section{Conference contributions}

- Bayoglu, R., Homminga, J., Koopman, B., Towards the development of a complete and coherent musculoskeletal model of the spine, $5^{\text {th }}$ Dutch Bio-Medical Engineering Conference, Egmond aan Zee, the Netherlands, 2015.

- Bayoglu, R., Geeraedts, L., Groenen, K.H.J., Verdonschot, N., Koopman, B., Homminga, J., How valid are left-right symmetrical musculoskeletal models?, $22^{\text {th }}$ Congres of the European Society of Biomechanics, Lyon, France, 2016.

- Bayoglu, R., Geeraedts, L., Groenen, K.H.J., Verdonschot, N., Koopman, B., Homminga, J., Are left-right symmetrical musculoskeletal models realistic?, $6^{\text {th }}$ Dutch Bio-Medical Engineering Conference, Egmond aan Zee, the Netherlands, 2017.

- Bayoglu, R., Geeraedts, L., Groenen, K.H.J., Verdonschot, N., Koopman, B., Homminga, J., A complete and coherent musculoskeletal dataset for the thoracic and cervical regions of the human spine, $23^{\text {th }}$ Congres of the European Society of Biomechanics, Seville, Spain, 2017.

- Bayoglu, R., Geeraedts, L., Groenen, K.H.J., Verdonschot, N., Koopman, B., Homminga, J., A complete and coherent musculoskeletal dataset for the human spine, $26^{\text {th }}$ Congress of the International Society of Biomechanics, Brisbane, Australia, 2017.

- Bayoglu, R., Galibarov E.P., Verdonschot, N., Koopman, B., Homminga, J., Twente Spine Model: A study of the intervertebral disc forces in flexion, Virtual Physiological Human Conference, Zaragoza, Spain, 2018. 


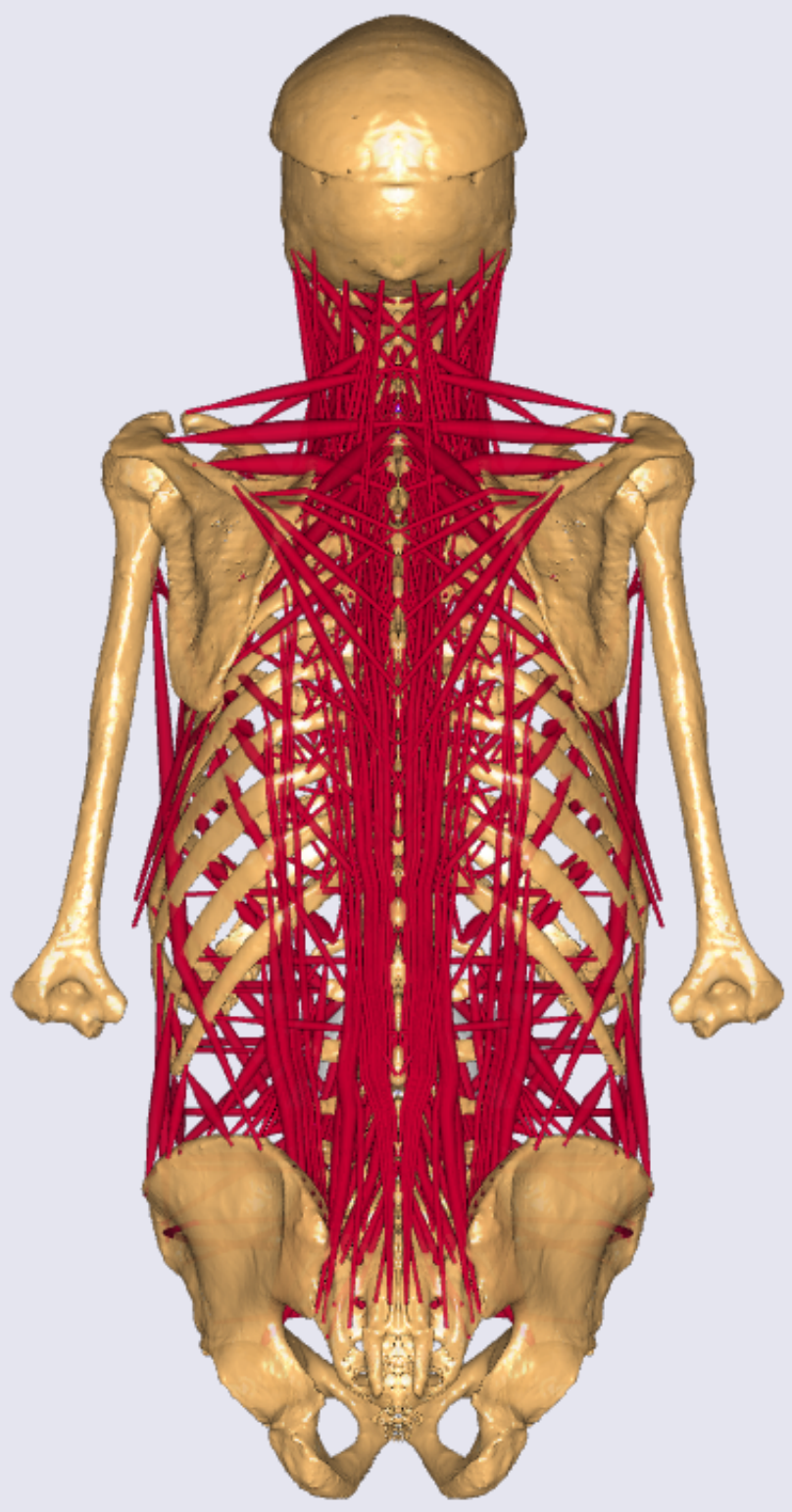

